\title{
The Effect of Blade Row \\ Interaction on Rotor Film Cooling
}

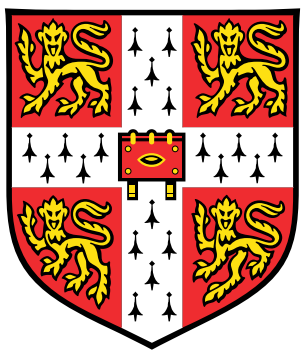

James Brind

Whittle Laboratory

Department of Engineering

University of Cambridge

This dissertation is submitted for the degree of

Doctor of Philosophy 



\section{Declaration}

The research presented in this dissertation was conducted at the Whittle Laboratory, Department of Engineering, University of Cambridge between October 2015 and March 2019. I hereby declare that except where specific reference is made to the work of others, the contents of this dissertation are original and have not been submitted in whole or in part for consideration for any other degree or qualification in this, or any other university. This dissertation is my own work and contains nothing which is the outcome of work done in collaboration with others, except as specified in the text and Acknowledgements. This dissertation contains fewer than 65,000 words including appendices, references, footnotes, tables and equations and has fewer than 150 figures.

James Brind

March 2019

\section{Publication}

Material from Chapters 5 and 6 of this dissertation has formed the following publication, due to be presented at the ASME Turbo Expo, Phoenix, Arizona, US in June 2019:

Brind, J., and Pullan, G. (2019). Effect of Blade Row Interaction on Rotor Film Cooling. ASME Paper No. GT2019-90961. Recommended for publication in the Journal of Turbomachinery. 



\title{
The Effect of Blade Row Interaction on Rotor Film Cooling
}

\author{
James Brind
}

In gas turbines, film cooling is required to protect metal parts from hot combustion gases. Reduction in coolant mass flow increases cycle efficiency, and hence reduces greenhouse gas emissions. However, the lifespan of a cooled component is sensitive to the metal temperature within the part. A designer requires predictions of cooling performance to make this compromise, yet present design methods are subject to uncertainty and are not viable without empirical input.

Flow through a turbine is inherently unsteady due to relative motion of stators and rotors, termed blade row interaction. Blade row interaction is not captured in flat-plate and cascade testing, or present design methods, contributing to uncertainty in predicted cooling performance. The aims of this thesis are to establish the mechanisms by which blade row interaction affects rotor film cooling, and quantify their influence on cooling performance in a representative case.

A new experimental rig is developed to facilitate aerodynamic and heat transfer measurements of cooling holes subject to unsteady main-stream boundary conditions. The effect of unsteadiness is set by non-linearity in the hole response. Unsteadiness reduces film effectiveness by up to $31 \%$ with cylindrical holes at a low momentum flux ratio, because the response to perturbations is non-linear. Cylindrical holes at a high momentum flux ratio, and fan-shaped holes, are robust to unsteadiness because they respond linearly.

Non-film-resolved computations are used to identify the blade row interaction mechanisms generating unsteady main-stream boundary conditions in a turbine rotor. A quasi-steady model is used to predict instantaneous excursions in cooling hole momentum flux ratio. Fluctuations of at least $\pm 30 \%$ are present for all hole locations, due to both upstream vane wake and potential field interaction.

A hybrid URANS-LES computational approach is implemented, validated against experimental data, and applied to a turbine stage cascade model. Compared to steady conditions, blade row interaction reduces rotor film effectiveness: by up to $18 \%$ on the pressure side, due to migration of vane coolant across the passage; and by up to $30 \%$ on the suction side, due to wake interactions increasing the film mixing rate. 



\section{Acknowledgements}

I am fortunate to have had the opportunity to pursue postgraduate study at the Whittle Laboratory, because of the many people who make it a productive, stimulating, and inspiring environment.

First, I would like to thank my Supervisor, Dr. Graham Pullan. His help and encouragement have been most valuable, while carefully measured, in order to foster my development as an independent researcher. I am very grateful for his guidance throughout this project. I would also like to thank my Advisor, Dr. Nick Atkins, for his interest in the work, and for sharing his expertise in heat transfer through insightful comments and suggestions.

The experiments described in this thesis were achieved with the help of the Whittle Laboratory Workshop. I am particularly grateful to Mr. John Saunders, for his endless patience and his profoundly wise advice; and Mr. Les Chapman for skilfully completing manufacture of the apparatus. I also thank Dr. Sam Grimshaw for lending me some of his instrumentation.

This project could not have been undertaken without the generous support of my industrial sponsor, Mitsubishi Heavy Industries. In addition to funding, they have offered a distinctly practical perspective, kept me personally motivated, and provided excellent hospitality on visits to Japan. In particular, I would like to thank Dr. S. Aoki, Dr. S. Uchida, Dr. E. Ito, Mr. H. Kitagawa, and Mr. Y. Kimura.

I am grateful for the year I spent with the MRes in Gas Turbine Aerodynamics cohort, not only for the well-rounded Turbomachinery education, but for the firm friendships established in the process. I would also like to thank the Crossword Team of Max, Tim, and Michael for enabling many hours of tea-time distraction.

Finally, I would like to thank my fiancée, Claire, for always being so kind to me, and my family, for giving me the best possible start in life. 



\section{Table of contents}

List of figures $\quad$ xi

Nomenclature $\quad$ Xv

1 Introduction 1

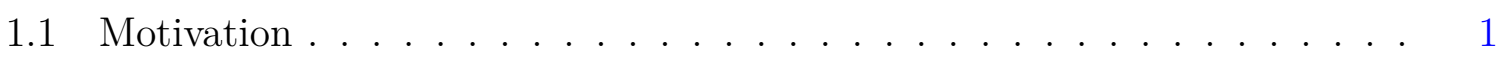

1.2 Research questions and approach . . . . . . . . . . . . . . 5

1.3 Layout of the thesis . . . . . . . . . . . . . . . 6

2 Literature review $\quad 7$

2.1 Introduction . . . . . . . . . . . . . . . . 7

2.2 Turbine blade row interaction . . . . . . . . . . . . . . 9

2.3 Film cooling on turbine rotors . . . . . . . . . . . . . . 12

2.4 Film cooling with artificial unsteadiness . . . . . . . . . . . . . 14

2.5 Computational modelling of film cooling . . . . . . . . . . . . . 18

2.6 Summary of literature and scope of the thesis . . . . . . . . . . . 23

3 Experimental methods $\quad 25$

3.1 Rig design and flow similarity . . . . . . . . . . . . . . . 25

3.2 Aerodynamic measurements . . . . . . . . . . . . . . . . . . 29

3.3 Heat transfer measurements . . . . . . . . . . . . . . . . . . 35

3.4 Summary of measurement uncertainties . . . . . . . . . . . . 45

4 Computational methods $\quad 49$

4.1 Flow solver . . . . . . . . . . . . . . . . . . 49

4.2 Non-film-resolved simulations . . . . . . . . . . . . . . . 50

4.3 Hybrid URANS-LES implementation . . . . . . . . . . . . . . . 54

4.4 Film-resolved flat plate simulations . . . . . . . . . . . . . . . 57

4.5 Film-resolved cascade simulations . . . . . . . . . . . . . 62 
5 Measurements of film cooling under unsteady boundary conditions 69

5.1 Introduction . . . . . . . . . . . . . . . . . . . 70

5.2 Unsteadiness amplitude . . . . . . . . . . . . . . . . . . 72

5.3 Film effectiveness . . . . . . . . . . . . . . . . . . . . . . . 74

5.4 Velocity field . . . . . . . . . . . . . . . . . . 83

5.5 Conclusions . . . . . . . . . . . . . . . . . . . 90 90

6 Non-film-resolved blade row interaction simulations $\quad 93$

6.1 Introduction . . . . . . . . . . . . . . . . . . . . . . 94

6.2 Conceptual approach . . . . . . . . . . . . . . . . . . . . 94

6.3 Mechanisms of blade row interaction . . . . . . . . . . . . . . . 97

6.4 Hole response modelling . . . . . . . . . . . . . . . . . . . . . . 107

6.5 Recommendations for design . . . . . . . . . . . . . . . 115

6.6 Conclusions . . . . . . . . . . . . . . . . . . 118

7 Film-resolved flat plate simulations $\quad 121$

7.1 Introduction . . . . . . . . . . . . . . . . . . . . . . 122

7.2 Boundary layer . . . . . . . . . . . . . . . . . . . . . 124

7.3 Cylindrical hole . . . . . . . . . . . . . . . . . . . . . 127

7.4 Shaped hole . . . . . . . . . . . . . . . . . . . . . 134

7.5 Conclusions . . . . . . . . . . . . . . . . . . . . . 141

8 Film-resolved cascade simulations $\quad 143$

8.1 Introduction . . . . . . . . . . . . . . . . . . . . . . . . . . . . . . 144

8.2 Convergence and resolution assessment . . . . . . . . . . . . . . 145

8.3 Time-averaged flow field . . . . . . . . . . . . . . . . . 153

8.4 Unsteady flow field . . . . . . . . . . . . . . . . . . 158

8.5 Assessment of quasi-steady framework . . . . . . . . . . . . . . 165

8.6 Conclusions . . . . . . . . . . . . . . . . . . . . . . . 168

9 Conclusions and recommendations for future work $\quad 171$

9.1 Conclusions . . . . . . . . . . . . . . . . . . . . . . . 171

9.2 Recommendations for future work . . . . . . . . . . . . . . . 175

$\begin{array}{ll}\text { References } & 179\end{array}$ 


\section{List of figures}

1.1 Turbine inlet temperature and efficiency of MHI gas turbines . . . . . . 2

1.2 Schematic diagram of an industrial gas turbine . . . . . . . . . . . . . . . . . . . . .

1.3 Cooling system of a high-pressure turbine blade . . . . . . . . . . . . . . 4

2.1 Schematic of turbine blade row interaction mechanisms. . . . . . . . . . . 10

2.2 Schematic of negative jet interaction . . . . . . . . . . . . . . . 11

2.3 Unsteady rotor surface static pressure distributions, simulations by Abhari and Epstein (1994) . . . . . . . . . . . . . . . . . . 13

2.4 Effect of blade row interaction on rotor film effectiveness, simulations by Abhari (1996) . . . . . . . . . . . . . . . . . . . . . 14

2.5 Effect of steady main-stream turbulence and unsteady wakes on film effectiveness, measurements by Saumweber and Schulz (2012) . . . . . . . 17

2.6 Computational predictions of film effectiveness, Fujimoto (2012) . . . . . 20

3.1 Schematic of the Unsteady Film Cooling Rig . . . . . . . . . . . . . 26

3.2 Design of the aerodynamic measurement probes . . . . . . . . . . . . . 34

3.3 Typical transient linear regression measurement data . . . . . . . . . . . 39

3.4 Comparison of film effectiveness distributions measured in Unsteady Film Cooling Rig to Saumweber et al. (2003) data . . . . . . . . . . . . . . . . 41

3.5 Measured precision error and confidence interval in film effectiveness . . . 43

3.6 Measured precision error and confidence interval in heat transfer coefficient 44

4.1 Meridional view of 1.5-stage turbine domain and boundary conditions . . 51

4.2 Block topology for 1.5-stage turbine computations . . . . . . . . . . . . 52

4.3 Grid points for 1.5-stage turbine computations . . . . . . . . . . . . 53

4.4 Computational zones in hybrid URANS-LES approach . . . . . . . . . . 55

4.5 Domain and boundary conditions for flat plate film cooling cases . . . . . 58

4.6 Cylindrical cooling hole mesh on $y / D=0$ plane . . . . . . . . . . . 60

4.7 Cylindrical cooling hole mesh on $z / D=0$ plane . . . . . . . . . . . 60 
4.8 Detail of hole inlet and exit mesh on $z / D=0$ plane . . . . . . . . . 61

4.9 Domain and boundary conditions for film-resolved cascade computation . 63

4.10 Schematic of O-mesh topology for vane wake refinement . . . . . . . . 65

4.11 Schematic of nested mesh topology for wall refinement . . . . . . . . . 65

4.12 Optimised block topology for film-resolved rotor blade . . . . . . . . . . . 67

5.1 Hole geometries for experimental tests after Saumweber et al. (2003) . . . 71

5.2 Measurements of main-stream unsteadiness amplitude . . . . . . . . . . . 73

5.3 Film effectiveness for cylindrical holes under steady conditions . . . . . . 75

5.4 Film effectiveness for cylindrical holes under unsteady conditions . . . . . 77

5.5 Effect of unsteadiness on laterally-averaged effectiveness, cylindrical holes 78

5.6 Momentum flux ratio characteristic of cylindrical holes . . . . . . . . . 78

5.7 Film effectiveness for shaped holes under steady conditions . . . . . . . . 80

5.8 Film effectiveness for shaped holes under unsteady conditions . . . . . . 81

5.9 Effect of unsteadiness on laterally-averaged effectiveness, shaped holes . . 82

5.10 Momentum flux ratio characteristic of shaped holes . . . . . . . . . . . . 82

5.11 Comparison of steady and unsteady velocity fields, nominal $I R=0.2 \quad$. $\quad 86$

5.12 Comparison of steady and unsteady velocity fields, nominal $I R=0.6 \quad$. $\quad 87$

5.13 Comparison of steady and unsteady velocity fields, nominal $I R=1.0 \quad$. 88

5.14 Effect of unsteadiness on laterally-averaged velocity profiles . . . . . . . . 89

6.1 Unsteady film cooling reduced-order modelling approach . . . . . . . . . 95

6.2 Instantaneous velocity perturbations in rotor passage, simulated . . . . . 98

6.3 Oscillation of rotor leading-edge stagnation point, simulated . . . . . . . 99

6.4 Migration of vane coolant due to blade row interaction, simulated . . . . 100

6.5 Static pressure perturbations in rotor due to potential field interaction - 102

6.6 Reduction of rotor passage to one-dimensional model . . . . . . . . . . . 104

6.7 Comparison of surface pressure fluctuations predicted by URANS and the one-dimensional passage model . . . . . . . . . . . . . . . . . . . 104

6.8 Streamline-normal pressure gradient fluctuation in rotor, simulated . . . 106

6.9 Schematic of the modifications to upstream vane . . . . . . . . . . . . 108

6.10 Vane exit non-uniformity profiles with different modified vanes . . . . . . 109

6.11 Blade cooling hole locations for reduced-order modelling . . . . . . . . . 109

6.12 Breakdown of linear and non-linear contributions to effect of blade row interaction on momentum flux ratio . . . . . . . . . . . . . . . . . . . . 112

6.13 Instantaneous momentum flux ratios predicted by hole response model . 114

6.14 Effect of velocity triangle design on negative jet interactions . . . . . . . 117 
7.1 Hole geometries for film-resolved computations . . . . . . . . . . . . . . 123

7.2 Development of flat plate boundary layer, hybrid URANS-LES . . . . . . 125

7.3 Boundary-layer velocity profiles, simulated . . . . . . . . . . . . 126

7.4 Stagnation temperature field on $z / D=0$, cylindrical hole, simulated . . . 128

7.5 Velocity field on $z / D=0$, cylindrical hole, simulated . . . . . . . . . . 129

7.6 Visualisation of vortical structure using Q-criterion, cylindrical hole . . . 130

7.7 Film effectiveness on $y / D=0$ wall, cylindrical holes, simulated . . . . . . 132

7.8 Predicted lateral distributions of film effectiveness compared to experimental data of Saumweber et al. (2003) . . . . . . . . . . . . . 133

7.9 Predicted streamwise distributions of laterally-averaged film effectiveness, cylindrical hole, compared to Saumweber et al. (2003) data . . . . . . . . 133

7.10 Effect of URANS layer thickness on film effectiveness prediction accuracy 135

7.11 Stagnation temperature field on $z / D=0$, shaped hole, simulated . . . 136

7.12 Velocity field on $z / D=0$, shaped hole, simulated . . . . . . . . . . . 137

7.13 Predicted hole discharge coefficients compared to Saumweber et al. (2003) 138

7.14 Film effectiveness on $y / D=0$ wall, shaped holes, simulated . . . . . 139

7.15 Predicted streamwise distributions of laterally-averaged film effectiveness, shaped hole, compared to Saumweber et al. (2003) data . . . . . . . . . . 140

8.1 Cooling hole locations on rotor blade cascade . . . . . . . . . . . . . . 145

8.2 Simulated convergence history of film-resolved cascade mass flow . . . . . 146

8.3 Ensemble averaging of rotor cooling hole momentum flux ratio . . . . . . 148

8.4 Ensemble averaging of rotor laterally-averaged film effectiveness . . . . . 148

8.5 Non-dimensional cell sizes in wall units for cascade . . . . . . . . . . . . 150

8.6 Instantaneous lateral vorticity at cascade mid-span, simulated . . . . . . 152

8.7 Comparison of cascade and 3D URANS vane wake profiles . . . . . . . . 154

8.8 Effect of blade row interaction on laterally-averaged film effectiveness . . 155

8.9 Effect of blade row interaction on cascade momentum flux ratios . . . . . 156

8.10 Migration of vane coolant in rotor cascade, simulated . . . . . . . . . . . 158

8.11 Main-stream turbulence intensity over rotor blade surface, simulated . . . 159

8.12 Ensemble-averaged cascade rotor film effectiveness, simulated . . . . . . . 161

8.13 Space-time diagram of cascade rotor film effectiveness, simulated . . . . 164

8.14 Validation of reduced-order hole response model using resolved $I R$. . . . 166 



\section{Nomenclature}

\begin{tabular}{|c|c|c|}
\hline \multicolumn{3}{|c|}{ Roman symbols } \\
\hline$B R$ & Cooling hole blowing ratio, Equation (2.2c) & - ] \\
\hline$B$ & Bias error & \\
\hline$C I$ & Confidence interval, Equation (3.16) & \\
\hline$C_{\mathrm{d}}$ & Discharge coefficient, Equation (5.4) or (6.1) & \\
\hline$D R$ & Cooling hole density ratio, Equation (2.2a) & \\
\hline$D$ & Cooling hole diameter & {$[\mathrm{m}]$} \\
\hline$f$ & Vane passing or main-stream forcing frequency & {$[\mathrm{Hz}]$} \\
\hline$h$ & Heat transfer coefficient, Equation (3.10) & {$\left[\mathrm{W} \mathrm{m} \mathrm{m}^{-2} \mathrm{~K}^{-1}\right]$} \\
\hline$I R$ & Cooling hole momentum flux ratio, Equation (2.2d) or (3.2) & \\
\hline$L$ & Cooling hole length & [m] \\
\hline$\dot{m}$ & Mass flow rate & {$\left[\mathrm{kg} \mathrm{s}^{-1}\right]$} \\
\hline$M a$ & Mach number & \\
\hline $\mathrm{Nu}$ & Nusselt number & \\
\hline$P$ & Cooling hole pitch & {$[\mathrm{m}]$} \\
\hline$p$ & Pressure & {$[\mathrm{Pa}]$} \\
\hline$\dot{q}$ & Heat flux & {$\left[\mathrm{W} \mathrm{m}^{-2}\right]$} \\
\hline$R e$ & Reynolds number, Equation (3.1) & \\
\hline$r$ & Radial coordinate & [m] \\
\hline$S$ & Precision error & \\
\hline$T u$ & Turbulence intensity & {$[\%]$} \\
\hline$T$ & Temperature & {$[\mathrm{K}]$} \\
\hline$t$ & Time & s] \\
\hline$U$ & Total uncertainty, Equation (3.5) & \\
\hline$V$ & Velocity & {$\left[\mathrm{m} \mathrm{s}^{-1}\right]$} \\
\hline$x$ & Axial or streamwise coordinate & \\
\hline$y$ & Wall-normal coordinate & \\
\hline$z$ & Lateral coordinate & \\
\hline
\end{tabular}




\section{Greek symbols}

$\alpha$

$\varepsilon_{\mathrm{f}} \quad$ Film effectiveness, Equation (2.1)

$\kappa \quad$ Reduced frequency, Equation (2.3) or (3.3)

$\lambda \quad$ Thermal conductivity

$\Psi \quad$ Pressure fluctuation amplitude, Equation (3.4)

$\rho \quad$ Density

$\Theta \quad$ Non-dimensional stagnation temperature, Equation (6.3)

$\theta \quad$ Circumferential coordinate

$\xi \quad$ Medial axis coordinate

$\zeta \quad$ Surface coordinate

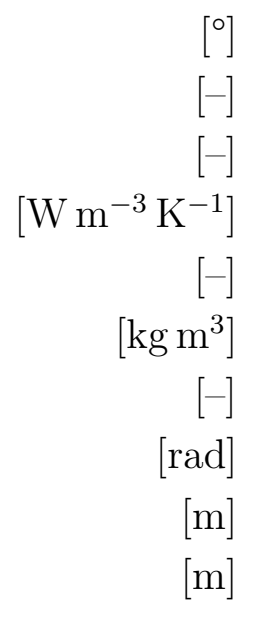

\section{Superscripts and accents}

$\begin{array}{ll}\text { rel } & \text { Rotor-relative frame } \\ * & \text { Gauge pressure } \\ + & \text { Wall units } \\ \square & \text { Laterally-averaged } \\ \hat{\square} & \text { Normalised to unity } \\ \square(t) & \text { Instantaneous } \\ \square^{\prime} & \text { Perturbation from mean } \\ \tilde{\square} & \text { Mean-to-peak fluctuation } \\ \langle\square\rangle & \text { Ensemble-averaged }\end{array}$

\section{Subscripts}

$\begin{array}{ll}\text { aw } & \text { Adiabatic wall conditions } \\ \text { c } & \text { Coolant conditions } \\ \text { hw } & \text { Hot-wire probe conditions } \\ \text { ref } & \text { Reference conditions } \\ \text { w } & \text { Wall conditions } \\ 0 & \text { Stagnation conditions } \\ \infty & \text { Main-stream conditions }\end{array}$

\section{Abbreviations}

CFD Computational Fluid Dyanmics

LES Large Eddy Simulation

(U)RANS (Unsteady) Reynolds-averaged Navier-Stokes 


\section{Chapter 1}

\section{Introduction}

\subsection{Motivation}

Natural gas accounted for $22 \%$ of global energy production in 2017 , according to the International Energy Agency (2018). Furthermore, their report suggests,

"Oil and natural gas will be part of the energy system for decades to come even under ambitious efforts to reduce greenhouse gas emissions."

(International Energy Agency, 2018)

The share of natural gas is projected to increase to $25 \%$ by 2040 ; in this scenario, energy demand is held constant, whilst emissions are reduced to achieve the global warming limitation targets set out in the United Nations Paris Agreement.

Natural gas may be burnt as fuel in a gas turbine. The energy released is converted to propulsive thrust in aircraft engines, or shaft power and electricity in land-based industrial machines. The future of the gas turbine is secure for two reasons: a requirement for high power density in civil aviation, and a need for responsive electricity generation capacity to balance intermittent renewable sources. The greenhouse gas emissions incurred in energy production are inversely proportional to the thermodynamic cycle efficiency. Therefore, increasing gas turbine efficiency is, and will remain, an important contribution to mitigating climate change.

The historical trend in the gas turbine industry is for higher firing temperatures, allowing higher cycle efficiency and increased specific work output. This is illustrated by the data for production Mitsubishi Heavy Industries gas turbines in Figure 1.1. Turbine inlet temperature has increased from $1420 \mathrm{~K}$ in the 'D-type' design, dating from the early 1980s, to $1880 \mathrm{~K}$ in the 'J-type', first commercial operation 2013. Combined-cycle thermal efficiency has improved from $49 \%$ to $61 \%$. 


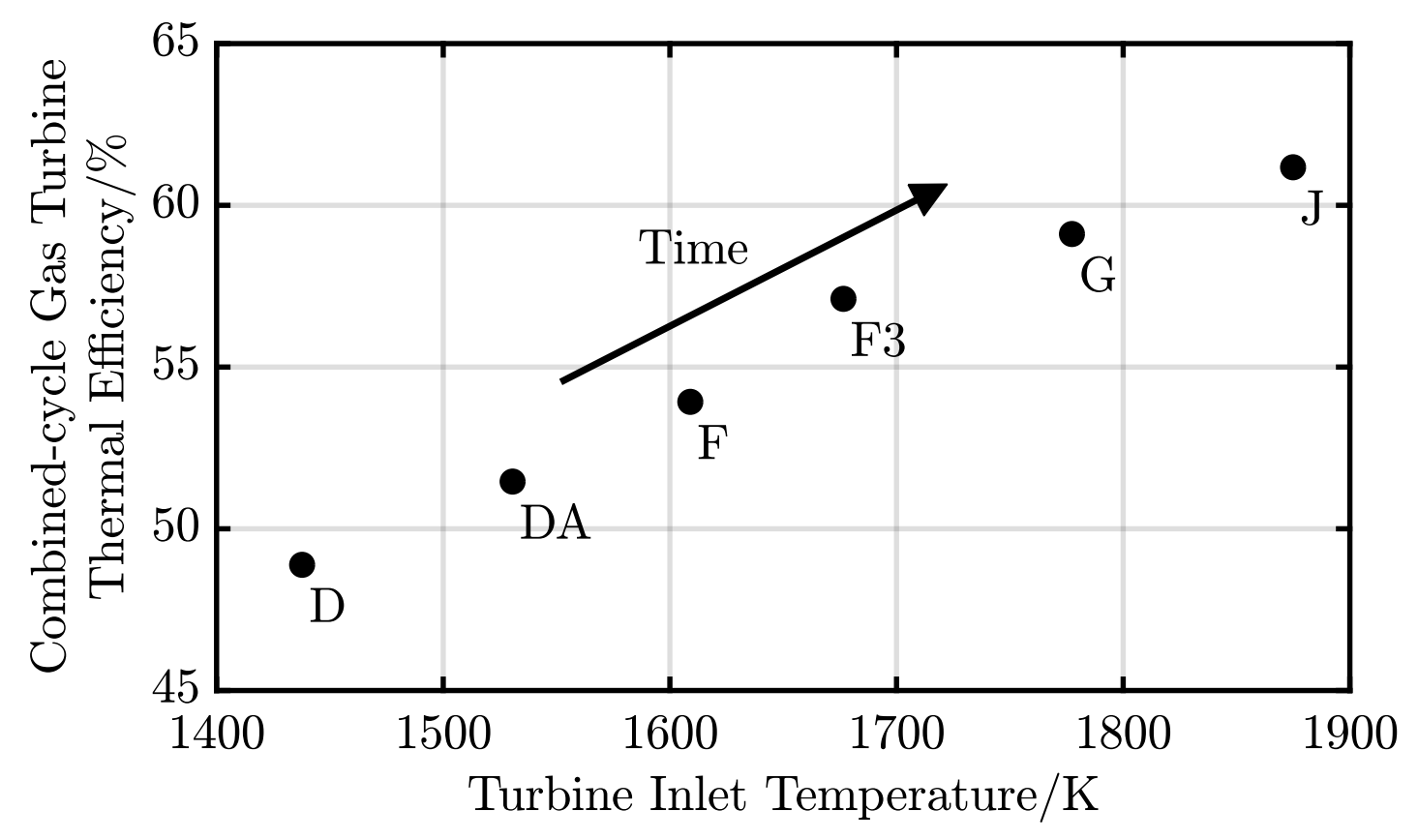

Fig. 1.1 Development of turbine inlet temperature and cycle efficiency of production Mitsubishi Heavy Industries gas turbines, from Yuri et al. (2013).

A simplified gas turbine cycle calculation (Cumpsty, 2003) confirms a thermodynamic underpinning for increase in cycle efficiency with turbine inlet temperature. However, this result ignores some practical complexities. In particular, raising the gas temperature above material limits requires active cooling to maintain acceptable metal temperatures and part lifespan. The benefit of a higher turbine inlet temperature must be balanced against losses due to cooling: detriment to the thermodynamic cycle due to extraction of coolant flow which bypasses the combustor, and reduction in aerodynamic efficiency of the turbine. Improvements in cooling performance allow a reduction in coolant flow at constant metal temperature, raising cycle efficiency.

Young and Wilcock (2002a,b) developed a thermodynamic cycle analysis code to predict gas turbine efficiency accounting for cooling losses, which was used by Wilcock et al. (2005) to quantify the benefit of improvements in cooling technology. Compared to a contemporary aero-derivative gas turbine, a "super-advanced" cooling configuration permits a reduction in coolant flow rate of approximately $50 \%$, and an increase in the combustor outlet temperature of $100 \mathrm{~K}$. The resultant increase in cycle efficiency is $1.2 \%$.

The component with the most stringent cooling requirements is the high-pressure turbine, marked on the schematic of an industrial gas turbine in Figure 1.2. Located immediately downstream of the combustor, the high-pressure turbine experiences the 


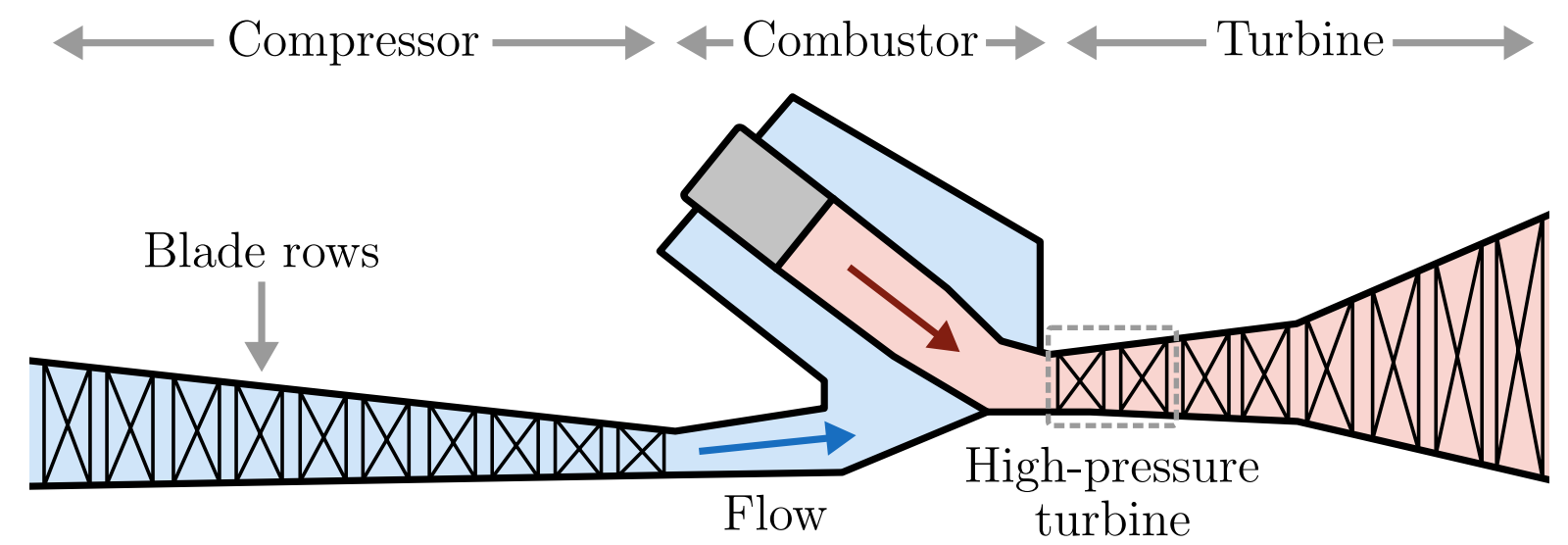

Axis of rotation

Fig. 1.2 Schematic diagram of the layout of an industrial gas turbine, with the highpressure turbine highlighted.

highest gas temperatures and convection velocities. The combination of heat load and centrifugal stress means that creep of the rotor blades sets the lifespan of the turbine (Bunker, 2017; Cumpsty, 2003).

The cooling system of a high-pressure turbine blade is shown in Figure 1.3. Coolant enters the blade at the root and is directed through serpentine internal passages, convectively cooling the inside of the blade. Geometric features are used to enhance heat transfer in this region: ribs, pin-fins, and impingement plates. Coolant is then ejected from the internal passages through holes in the blade surface to provide a protective external film of colder air: this is known as film cooling. There are of order 200 discrete film cooling holes on a high-pressure turbine blade. Coolant is also ejected at the blade trailing edge and tip. Film cooling is used to cool the vanes, blades, endwalls, and combustor in current gas turbines. A total coolant flow of order $15 \%$ of the machine inlet mass flow is representative of large industrial gas turbines.

Turbine cooling systems are not straightforward to design for two reasons. First, the success or failure of a design is sensitive to the actual metal temperature reduction achieved. According to Bogard and Thole (2006), an error of $25 \mathrm{~K}$ in metal temperature can change the component lifespan by a factor of two. Using the metal temperature data from Aoki (1999), an error of $25 \mathrm{~K}$ corresponds to predicting the cooling performance to better than $4 \%$. Second, predicting the performance of a particular design is subject to uncertainties, including unknown manufactured geometry, unknown turbine boundary conditions, and inaccurate modelling methods. 

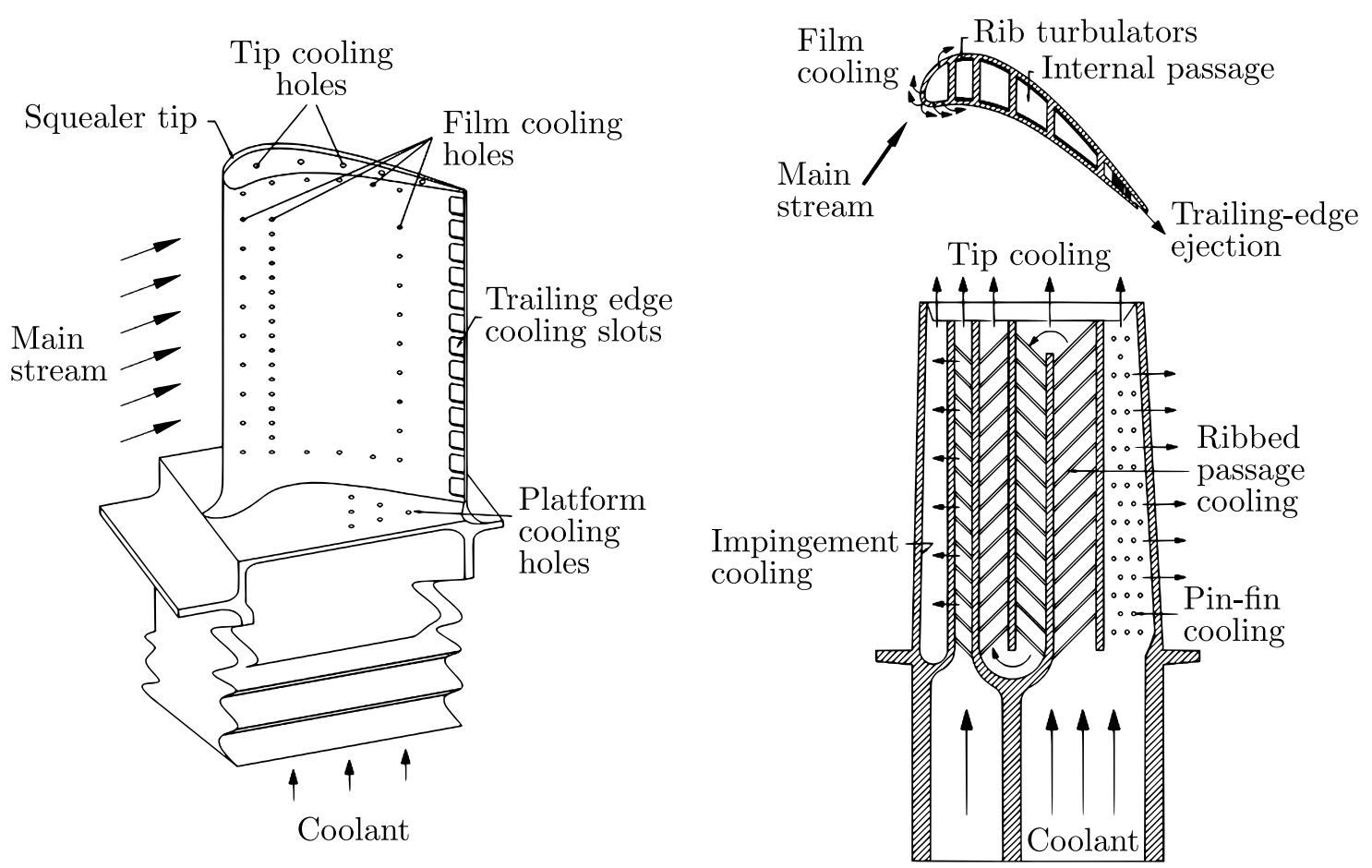

Fig. 1.3 Cooling system of a high-pressure turbine blade, adapted from Han (2004), licensed under CC BY 3.0.

In practice, these uncertainties are overcome using empiricism. Designs are based on previous experience, a generous safety margin is allowed, and coolant mass flow is increased if problems arise in a full-scale turbine test. The test results are incorporated into design tools via an entirely empirical 'data matching' process. Although pragmatic, this evolutionary approach has led to stagnation and conservatism in the field.

In a recent review of the state of the art, Bunker (2017) argues that quantification and reduction of unknowns is the greatest challenge in turbine cooling, and yet most current research is directed towards achieving high performance in idealised laboratory conditions. Bunker states,

"The practice of data matching side steps most unknowns... [and] can lead to complacency and a false comprehension that those unknowns have been eliminated."

(Bunker, 2017)

It is desirable to reduce prediction uncertainties, for a more robust design process, reducing the likelihood of expensive late-stage corrections to the cooling design. Furthermore, a better understanding of the fluid mechanics of the cooling system at turbine conditions 
gives rise to the possibility of improved designs, where increased performance allows a decrease in coolant flow and hence an increase in cycle efficiency.

Relative motion of stationary vanes and rotating blades leads to time-varying flow in a turbine rotor, termed blade row interaction. Unsteady flow is not present in laboratoryscale flat plate or cascade film cooling tests, nor captured in present design methods. However, the possibility for blade row interaction to affect rotor film cooling has been recognised since early measurements of film cooling performance on rotating blades showed discrepancies compared to predictions based on cascade and flat-plate measurements (discussed in the Literature review, Section 2.3). Bogard and Thole (2006) reviewed film cooling, noting that many experimental studies use a simple apparatus not representative of real turbine conditions. In particular, they suggest that conclusions drawn with low main-stream turbulence levels may not be generally applicable; a similar argument could be made for the importance of imposing representative unsteady boundary conditions. According to Bunker (2017), the performance resulting from unsteady flows remains an extant, unquantified unknown in turbine cooling.

\subsection{Research questions and approach}

The purpose of this thesis is to contribute to the understanding of the effects of blade row interaction on rotor film cooling. The particular turbine studied herein is representative of large industrial gas turbines, however, emphasis is placed on the general mechanisms as they apply to all machines. The work addresses three specific research questions:

1. What are the fluid-dynamic mechanisms that characterise blade row interaction effects on rotor film cooling?

2. How is the time-averaged unsteady film cooling different from the nominal steady film cooling?

3. How should blade row interaction effects on rotor film cooling be modelled?

A three-part approach is employed, including experimental measurements, low-fidelity computations, and high-fidelity computations. The experiments, using a simplified flat plate configuration, test the performance of cooling holes subject to main-stream unsteadiness. The low-fidelity computations, with turbine stage geometry and boundary conditions, allow characterisation of the unsteady turbine aerodynamics as they affect rotor film cooling. High-fidelity computations are used to conduct a virtual experiment quantifying the effect of blade row interaction on cooling performance in a representative cascade case. 


\subsection{Layout of the thesis}

This thesis is organised as follows. In Chapter 2, relevant literature is summarised to furnish background information and frame the scope of the present work. The experimental methods used are described in Chapter 3, and the computational methods in Chapter 4.

Experimental results, showing the effect of main-stream unsteadiness on film cooling performance, are presented in Chapter 5. Low-fidelity computations are used to study the blade row interaction mechanisms affecting rotor film cooling in Chapter 6 .

A high-fidelity computational approach is validated against experimental data in Chapter 7, before being applied in a turbine cascade virtual experiment in Chapter 8. Finally, conclusions are drawn, and suggestions for future work given, in Chapter 9. 


\section{Chapter 2}

\section{Literature review}

\subsection{Introduction}

The film cooling literature comprises a large number of publications, and remains an active area of research at the present time, with fifty related conference papers presented at ASME Turbo Expo 2018 (ASME, 2018). However, understanding of the effect of blade row interaction on film cooling remains limited. In particular, the implications for the design of rotor film cooling are not clear.

After a nomenclature, this review covers four topics. First, a synopsis of existing knowledge of turbine blade row interaction is presented. Second, investigations into film cooling on a rotating stage are discussed. Next, non-rotating film cooling experiments with artificial unsteadiness are considered. Finally, computational methods for modelling film cooling are reviewed. The chapter ends by summarising the literature in order to set out the scope of the new work presented in the thesis.

The film cooling nomenclature used in this Chapter and the remainder of this thesis is now defined. Cooling performance may be quantified non-dimensionally using film effectiveness, $\varepsilon_{\mathrm{f}}$, defined for a compressible flow,

$$
\varepsilon_{\mathrm{f}}=\frac{T_{\mathrm{aw}}-T_{\mathrm{rec}, \infty}}{T_{\mathrm{rec}, \mathrm{c}}-T_{\mathrm{rec}, \infty}},
$$

where $T_{\mathrm{aw}}$ is the adiabatic wall temperature (the driving temperature for convective heat transfer), and $T_{\text {rec, } \infty}$ and $T_{\text {rec,c }}$ are the main-stream and cooling hole exit recovery temperatures respectively. Film effectiveness is a measure of the local degree of mixing between coolant and main-stream flows, where $\varepsilon_{\mathrm{f}}=0$ corresponds to no cooling, and $\varepsilon_{\mathrm{f}}=1$ to maximum performance. Ejecting coolant from a row of discrete holes will result in lateral film effectiveness gradients across the hole pitch. However, the thermal 
conductivity of the metal surface is high enough that the overall effect on heat load is well-represented by an area-averaged value across the hole pitch, the laterally-averaged film effectiveness $\overline{\varepsilon_{\mathrm{f}}}$.

The metal temperature is not only a function of the adiabatic wall temperature, but is instead set by the heat flux through the blade wall. This means that the metal temperature is a function of the adiabatic wall temperatures and heat transfer coefficients on both external and internal sides of the blade wall. The total benefit of film cooling should be quantified taking into account variations in external heat transfer coefficient, as in the net heat flux reduction (Sen et al., 1996) or the "Delta Phi" (Rutledge et al., 2016) methods. Both these metrics require additional specific assumptions about the wall temperature or internal cooling flow. The scope of this thesis is restricted to changes in film effectiveness due to external aerodynamic blade row interactions.

The relation between main-stream and coolant flow conditions is fixed by a combination of two of the density, velocity, blowing, and momentum flux ratios,

$$
\begin{aligned}
D R & =\frac{\rho_{\mathrm{c}}}{\rho_{\infty}}, \\
V R & =\frac{V_{\mathrm{c}}}{V_{\infty}}, \\
B R & =\frac{\rho_{\mathrm{c}} V_{\mathrm{c}}}{\rho_{\infty} V_{\infty}}, \\
I R & =\frac{\rho_{\mathrm{c}} V_{\mathrm{c}}^{2}}{\rho_{\infty} V_{\infty}^{2}} .
\end{aligned}
$$

Coolant conditions are indicated by a subscript c, and main-stream conditions by a subscript $\infty$. The turbine designer has no direct influence on the density ratio, which is determined by the temperature rise through the combustor. The only control available is varying the coolant supply pressure, which will change the velocity, blowing, and momentum flux ratios simultaneously. A set of the parameters in Equations (2.2) will be referred to as a cooling hole operating point.

The blowing ratio is a non-dimensional mass flux through the cooling hole. The momentum flux ratio characterises the trajectory of coolant as it leaves the cooling hole. For example, with cylindrical cooling holes, coolant separates from the wall for $I R>0.8$ (Bogard and Thole, 2006). 


\subsection{Turbine blade row interaction}

The flow in a turbomachine is inherently unsteady. Neglecting viscous effects, the only way to exchange work with a fluid is to impose a non-zero temporal pressure gradient (Dean, 1959). A turbine creates these conditions with alternating stator and rotor rows. Flow unsteadiness in the reference frame of one row, caused by the relative motion of other rows, is termed blade row interaction.

Blade row interaction creates unsteadiness at relatively low, fixed, deterministic frequencies related to the number of blades in each row. A second type of unsteadiness is non-deterministic, relatively high-frequency, turbulent unsteadiness generated in mixing and shear layers. Non-deterministic unsteadiness is not the focus of this work; a statistical approach allows the effect of turbulence to be approximately modelled as a function of averaged properties such as turbulence intensity or length scale.

Any circumferential non-uniformity in the stationary frame, upstream of a rotating blade row, will lead to unsteadiness in the rotor-relative frame. Routine design simulations use a mixing plane between rows, where a circumferential averaging procedure enforces steady inlet conditions to each row, and hence do not capture blade row interaction. The following paragraphs discuss different blade row interaction mechanisms, illustrated schematically in Figure 2.1.

Potential field interaction The blockage and loading of a vane or blade row creates an inviscid pressure disturbance to the flow, a 'potential field'. The disturbance exists upstream and downstream, decaying exponentially on a length scale proportional to the circumferential pitch (Greitzer et al., 2004). The potential field of, say, a stator, is 'frozen' to the vanes, so that, in the rotor-relative frame, a blade experiences an approximately sinusoidal time-varying static pressure as the vane sweeps past. Kachel and Denton (2006) performed unsteady surface pressure measurements in a multi-stage, low-speed turbine. In addition to the frozen potential field, they also observed one-dimensional pressure waves, where fluctuations due to the frozen potential field propagate downstream through the rotor passage.

Wake interaction One of the first studies on the interaction between wakes and downstream rotating rows was performed by Meyer (1958), who proposed the negative jet model, shown on Figure 2.2. A wake is represented as a velocity perturbation to the background flow, acting in the direction of the velocity deficit. The perturbation persists within the rotor, and directs wake fluid towards the suction side of the blade. Hodson and Dawes (1998) provide a review of measurements and computations of wake interaction 


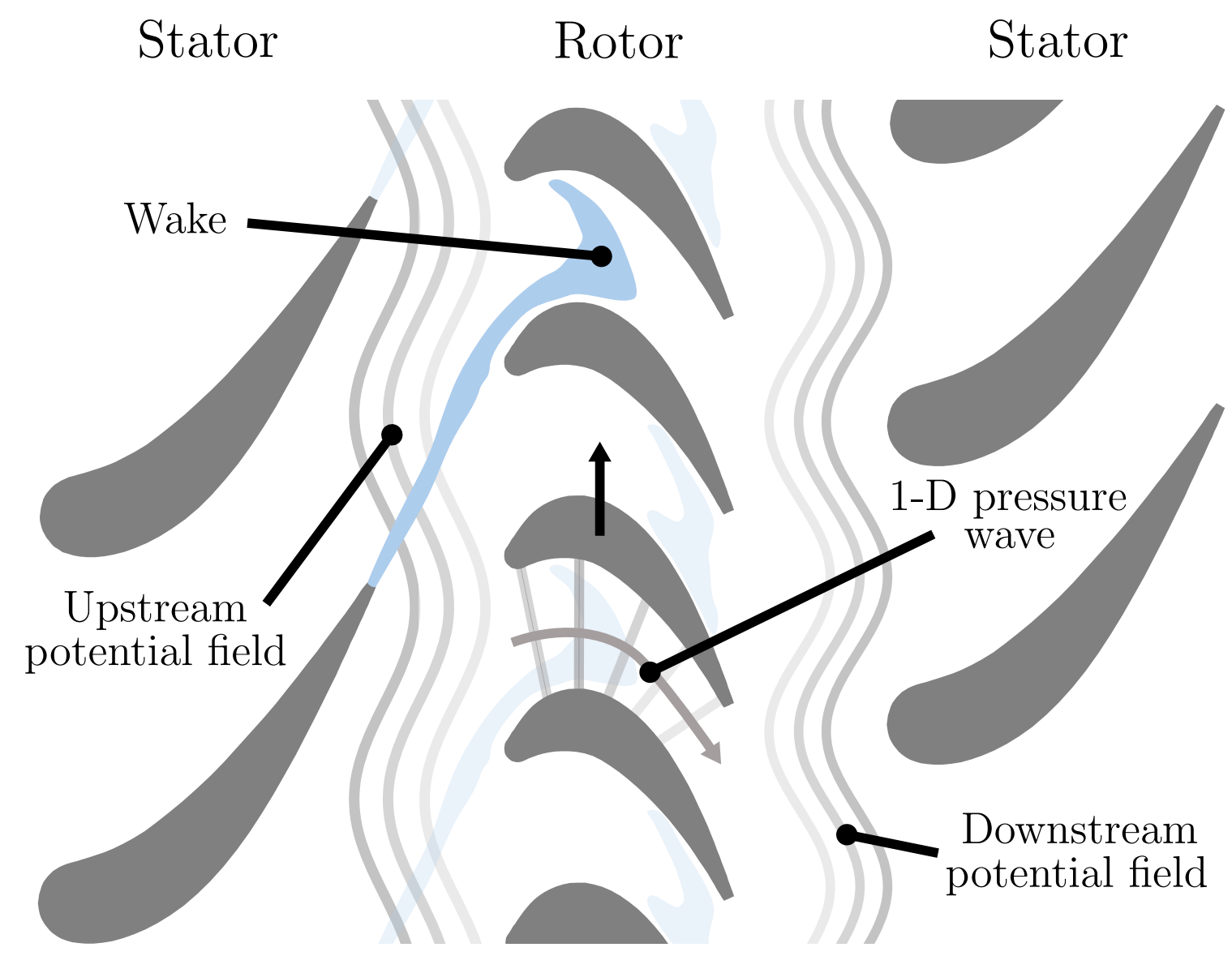

Fig. 2.1 Schematic of turbine blade row interaction mechanisms. 


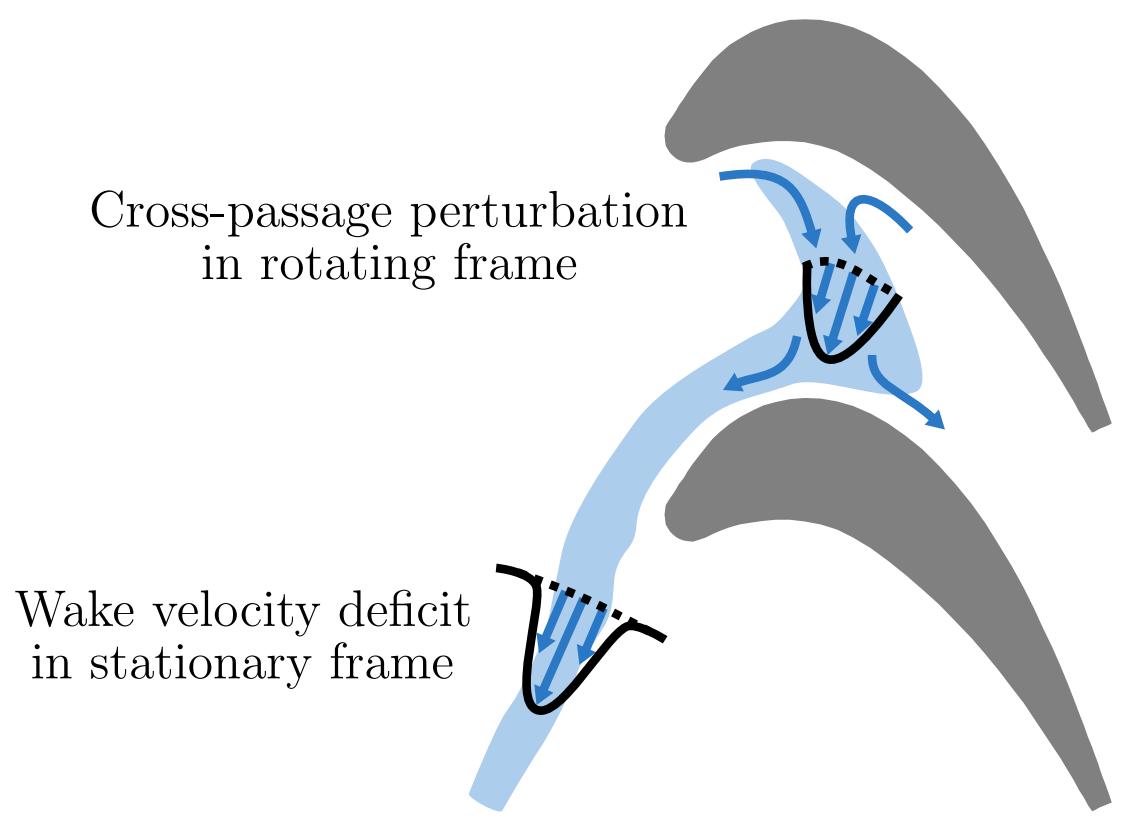

Fig. 2.2 Schematic of negative jet interaction in a turbine rotor (Meyer, 1958).

kinematics, which are all consistent with the negative jet model. Kachel and Denton (2006) asserted that, in addition to velocity fluctuations, the velocity deficit within the wake produces static pressure fluctuations due to unsteady streamline curvature effects.

Shock wave interaction If the vane exit flow is supersonic, shock waves are shed from vane trailing edges which impinge on, and reflect away from, downstream rotor blades (Giles, 1990). It was found by Miller et al. (2003), for their research turbine representative of civil aero-engines, that shock wave interactions produce pressure fluctuations an order of magnitude greater than wake and potential field interactions. It can therefore be expected that shock wave interactions will dominate in highly-loaded turbines with supersonic exit flow.

Secondary flow interaction The mechanisms described above are two-dimensional, in that they act on blade-to-blade surfaces. The presence of endwalls in a real threedimensional turbine causes secondary flow, where endwall boundary-layer vorticity is reoriented as it convects through the stator to acquire a component in the streamwise direction. This process produces non-uniformity in both the circumferential and radial directions. Examples of unsteady flow in a downstream rotor due to upstream vane 
secondary flow are reported by Binder (1985), Kachel and Denton (2006), and Pullan (2006).

Thermal interaction Temperature non-uniformity at rotor inlet may be caused by hot streaks originating from the combustor, or coolant from upstream vanes. A lower density causes cold vane wake fluid to acquire a reduced rotor-relative flow angle, which augments the wake negative jet, tending to convect coolant towards the suction side (Kerrebrock and Mikolajczak, 1970). Conversely, hot fluid acquires an increased flow angle and migrates towards the pressure side (Ong and Miller, 2012). Temperature non-uniformity also generates additional secondary flows through buoyancy effects and gradients in rotor-relative inlet conditions, which redistribute fluid within the blade passage, as reported by Butler et al. (1989).

\subsection{Film cooling on turbine rotors}

The first measurements of film cooling in a rotating-stage environment were performed by Dring et al. (1980). The film effectiveness distributions were compared with corresponding flat-plate data: a good agreement was seen on the suction side, but the pressure-side film effectiveness was reduced in the rotating case. Takeishi et al. (1992) reported a study of rotor film cooling conducted at Mitsubishi Heavy Industries, Takasago. Measurements of blade film effectiveness in a rotating rig were compared to measurements taken on a stationary linear cascade with the same geometry and cooling configuration. Film effectiveness levels were similar at the leading edge and near the suction-side hole, but far downstream on the suction side, film effectiveness was up to $30 \%$ lower in the rotating case. No data were presented for the pressure side.

Abhari and Epstein (1994) measured heat flux distributions on a rotating turbine stage in a transient facility at MIT, with full dynamic similarity to engine conditions. Results from a cooled rotor were compared with cooled cascade tests of the same profile. The suction-side heat flux to the rotor was lower by up to $60 \%$, and pressure-side data were not available. Abhari and Epstein (1994) also performed analytical modelling of the unsteady cooling behaviour. First, it is noted that blade surface static pressure fluctuations are of the same order as the nominal time-averaged coolant dynamic head, as shown by the surface static pressure results in Figure 2.3, which will cause fluctuations in coolant mass flow. A simple model for unsteady cooling was developed, where the instantaneous coolant mass flow is estimated and used as input to a flat-plate film effectiveness correlation, which is convected downstream. Then, the local instantaneous 


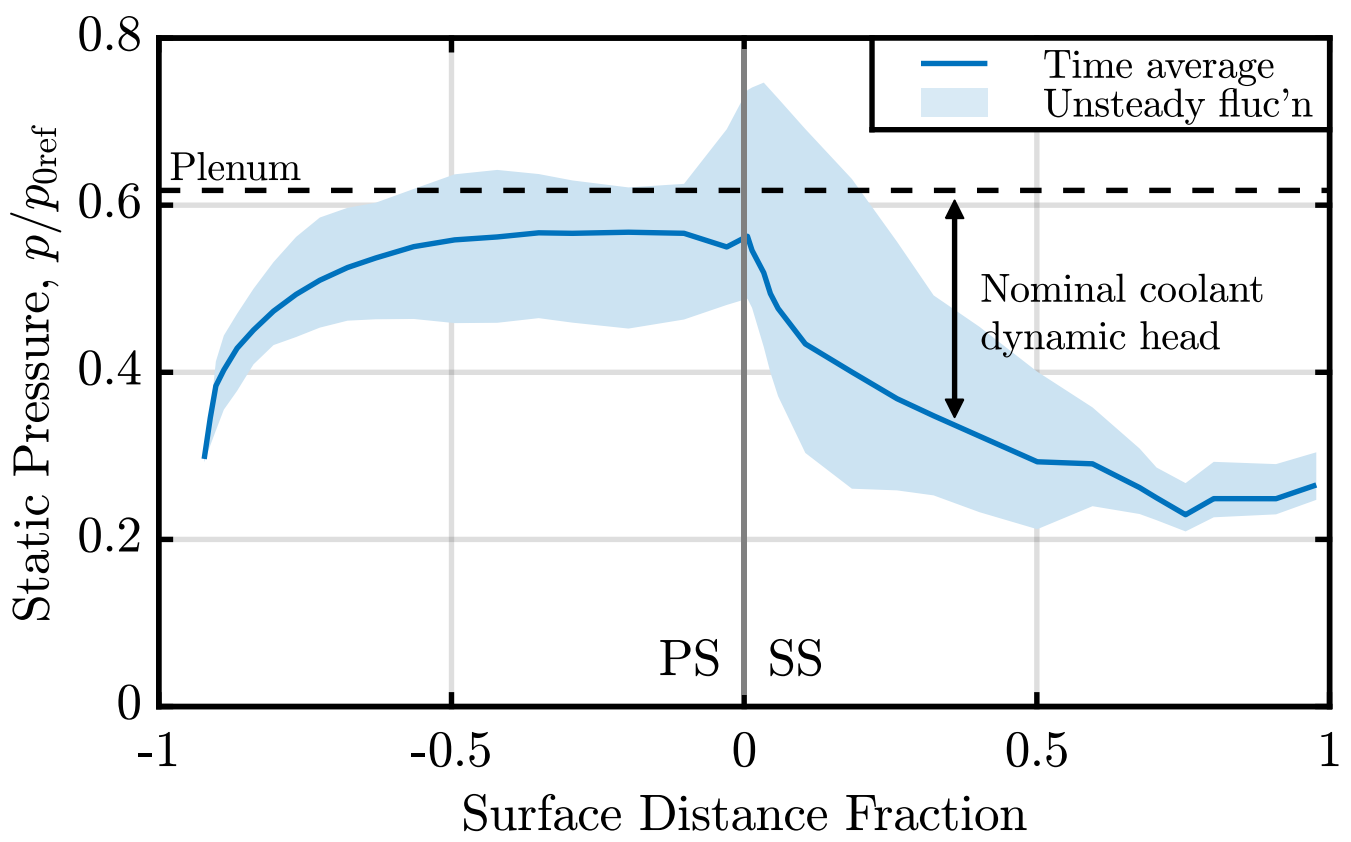

Fig. 2.3 Rotor surface static pressure distribution, turbine stage simulations by Abhari and Epstein (1994). The unsteady fluctuation in surface pressure is of the same order as the nominal coolant dynamic head available over the majority of the blade surface.

film effectiveness is used to correct measured values of uncooled heat flux. The model reproduces the qualitative features of the cooled heat flux data: film cooling reduces the time-averaged heat flux, and introduces a phase shift in the fluctuations. Comparing time-averaged unsteady heat fluxes, and heat fluxes using the time-averaged main-stream pressure as a boundary condition, the model predicts that unsteady effects reduce heat flux by $12 \%$ at the suction surface sensor location, and increase heat flux at the pressure surface sensor location by $5 \%$.

Further experiments and computations were performed by Abhari (1996) on the MIT turbine. A more complicated injection model, based on a series of correlations, was incorporated into a two-dimensional, unsteady, coupled Euler/Navier-Stokes computational fluid dynamics (CFD) solver. Two simulations were performed of the film-cooled turbine with steady (circumferentially mixed out) and unsteady rotor inflow respectively. The unsteady simulation predicts time-averaged heat fluxes on the pressure side up to a factor of two higher than the steady-state simulation, due to a reduction of up to $64 \%$ in film effectiveness, shown in Figure 2.4. After matching the time-averaged coolant mass flow, the remaining $45 \%$ of this discrepancy is attributed to unsteady effects. Time-averaged 


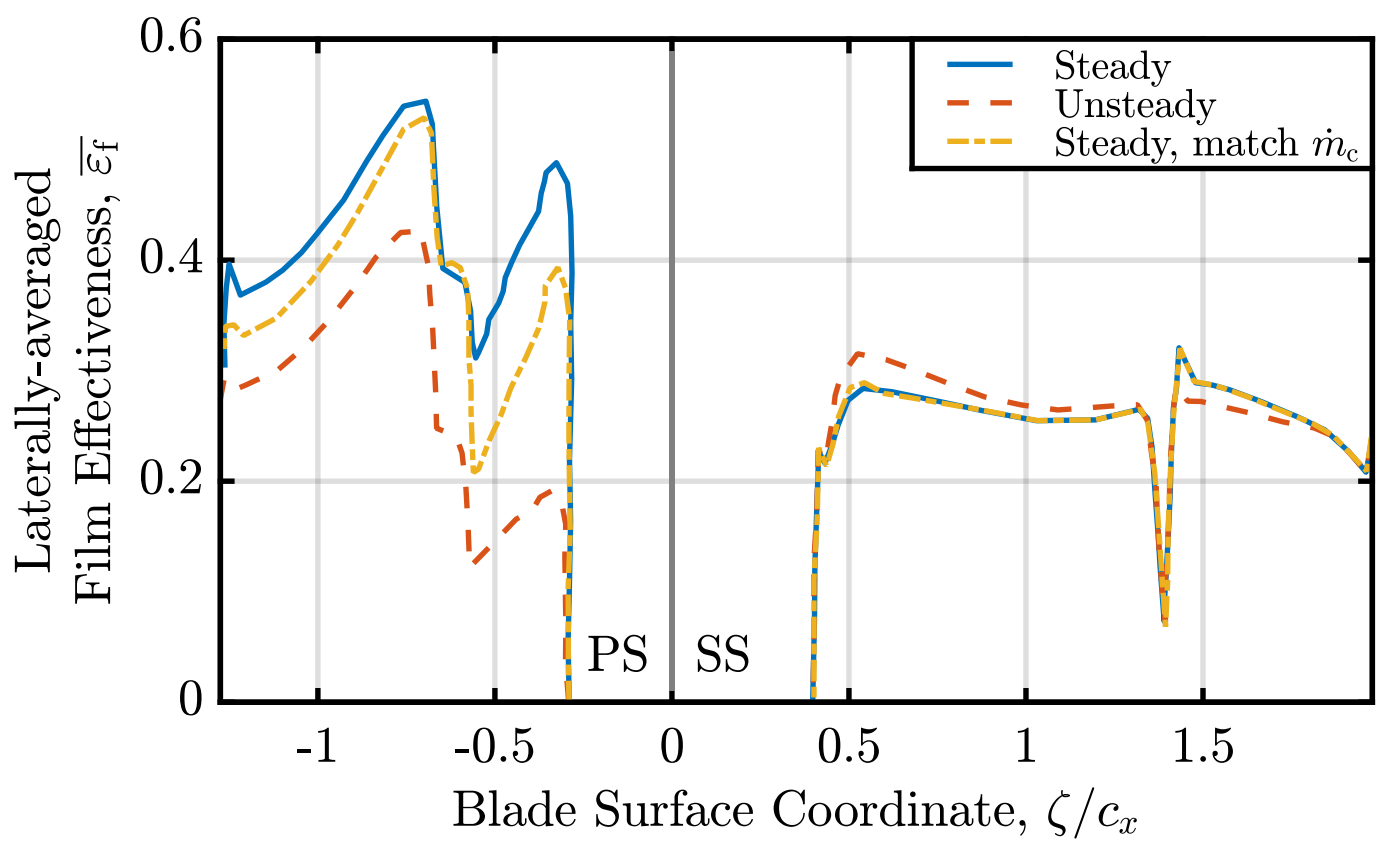

Fig. 2.4 Rotor film effectiveness distributions, predicted by Abhari (1996). Comparison of steady, time-averaged unsteady, and steady simulation with hole mass flows matched to unsteady values. Unsteadiness reduces film effectiveness by up to $64 \%$.

heat fluxes on the suction side match to within $5 \%$, with all the discrepancy due to unsteady effects alone.

The MIT work is important because it demonstrates the mechanism of unsteady coolant flow affecting instantaneous rotor film cooling performance. However, the authors state that the cooling hole arrangement "was chosen to provide the maximum information on cooling performance in rotating geometries rather than be representative of a production blade cooling configuration". The authors also suggest that because the test turbine is highly-loaded, the effects are exaggerated compared to lightly-loaded designs.

\subsection{Film cooling with artificial unsteadiness}

Due to the mechanical complexity of rotating rigs, and of acquiring measurements in the rotor-relative frame, the effect of unsteady boundary conditions on film cooling has been more commonly investigated using non-rotating flat-plate and cascade experiments with artificial unsteadiness. The boundary conditions imposed are necessarily a particular idealisation of real blade row interaction. The role of reduced frequency and validity of 
the quasi-steady assumption will be discussed first. The discussion that follows will be subdivided according to the method of generating unsteadiness: moving-bar wakes, and static pressure fluctuations.

\subsubsection{Reduced frequency and quasi-steady flow}

The parameter that characterises the importance of unsteady effects in a given flow is the reduced frequency, $\kappa_{\mathrm{c}}$. For a rotor cooling hole, this can be defined as a ratio of the two characteristic time scales,

$$
\kappa_{\mathrm{c}}=\frac{\text { coolant convection time }}{\text { vane passing period }}=\frac{L / V_{\mathrm{c}}}{1 / f}=\frac{f L}{V_{\mathrm{c}}},
$$

where $L$ is the cooling hole length, $V_{\mathrm{c}}$ the coolant velocity, and $f$ the vane passing frequency. If the reduced frequency is high, $\kappa_{\mathrm{c}} \gg 1$, unsteady effects will dominate. If the reduced frequency is low, $\kappa_{\mathrm{c}} \ll 1$, the flow is quasi-steady, and the instantaneous unsteady flow is the same as a steady flow with the instantaneous boundary conditions.

If rotor cooling holes subject to blade row interaction behave quasi-steadily, this permits simplified modelling, because existing steady characterisations can be separately applied at each instant in time. Taking values for the parameters representative of a $50 \mathrm{~Hz}$ large industrial gas turbine, the reduced frequency is of order $\kappa_{\mathrm{c}} \approx 0.06$. This is a low value; therefore, the hole flow is expected to behave quasi-steadily for this case.

In addition to relative time-scale arguments, the quasi-steady assumption is also supported by measurements comparing steady and instantaneous unsteady aerodynamic fields. Ligrani et al. (1996) used flow visualisations of film cooling subject to main-stream static pressure oscillations to conclude that the coolant film behaves quasi-steadily for $\kappa_{\mathrm{c}} \leq 0.16$ at all momentum flux ratios. Bernsdorf et al. (2008) performed particle image velocimetry measurements of film cooling with imposed coolant mass flow pulsations, observing quasi-steady behaviour up to $\kappa_{\mathrm{c}}=0.03$ with fluctuations of $\pm 40 \%$ in momentum flux ratio.

\subsubsection{Moving-bar wakes}

The most common idealised boundary condition is an upstream moving-bar wake generator, to model the interaction between vane wakes and rotor film cooling. The following studies all concern cylindrical cooling holes. On a cascade rig, the group at Texas A\&M University found a consistent decrease in film effectiveness due to wake interaction, of $20 \%$ to $30 \%$, across all tested blowing and density ratios, with and without wake 
coolant (Du et al., 1999, 1998; Mehendale et al., 1994). Funazaki et al. (1997) also found that, for their leading edge model, wakes are detrimental, with low-blowing-ratio films at $B R=0.4$ most susceptible, where film effectiveness reduces by $20 \%$. When the main-stream turbulence intensity is increased from $1 \%$ to $4 \%$ via a turbulence grid, wakes have a negligible additional effect. In their annular cascade tests, Heidmann et al. (2001) found that the reduction in film effectiveness due to wakes is constant in the streamwise direction, and proportional to the wake reduced frequency. It has been reported that it is possible for the addition of passing wakes to increase the film cooling effectiveness of a cylindrical hole operating at a high momentum flux ratio, when the jet would separate from the surface in steady state. Womack et al. (2008) measure a $100 \%$ increase in film effectiveness for $I R=1$; Saumweber and Schulz (2012) measure a $60 \%$ increase at $I R=1.3$.

For fan-shaped cooling holes, Saumweber and Schulz (2012) found bar wakes to be detrimental at all momentum flux ratios, reducing film effectiveness by up to $45 \%$ at low momentum flux ratio and up to $19 \%$ at high momentum flux ratio.

Qualitatively, the effect of wakes is identical to an elevated steady main-stream turbulence level, demonstrated by the data from Saumweber and Schulz (2012) for low-turbulence, high-turbulence and unsteady wake conditions in Figure 2.5. Data are presented for cylindrical holes, Figure 2.5(a), and fan-shaped holes, Figure 2.5(b), each at two blowing ratios. The extra mixing associated with turbulence is detrimental to cooling performance unless the coolant is detached from the surface, as with a cylindrical hole at high momentum flux ratio, $B R=1.5$ in Figure 2.5(a). In all other cases, high main-stream turbulence and unsteady wakes reduce effectiveness by similar amounts, up to $40 \%$, suggesting that the wake effect is linear, and could be modelled with a time-averaged turbulence intensity.

\subsubsection{Static pressure fluctuations}

A different idealised boundary condition is a fluctuation in main-stream or coolant static pressure, simulating potential field interactions occurring in a real turbine. An advantage of this form of unsteadiness is that the time-averaged boundary conditions can be held constant, isolating non-linear unsteady effects. This is not possible with moving-bar rigs, where adding wakes introduces unsteadiness and also increases the time-averaged turbulence intensity.

Ligrani et al. (1996) investigated the effects of main-stream static pressure pulsations, generated by downstream rotating shutters, on cylindrical film cooling holes operating at low momentum flux ratio $I R=0.06$. At a quasi-steady reduced frequency, $\kappa_{\mathrm{c}}=0.1$, 

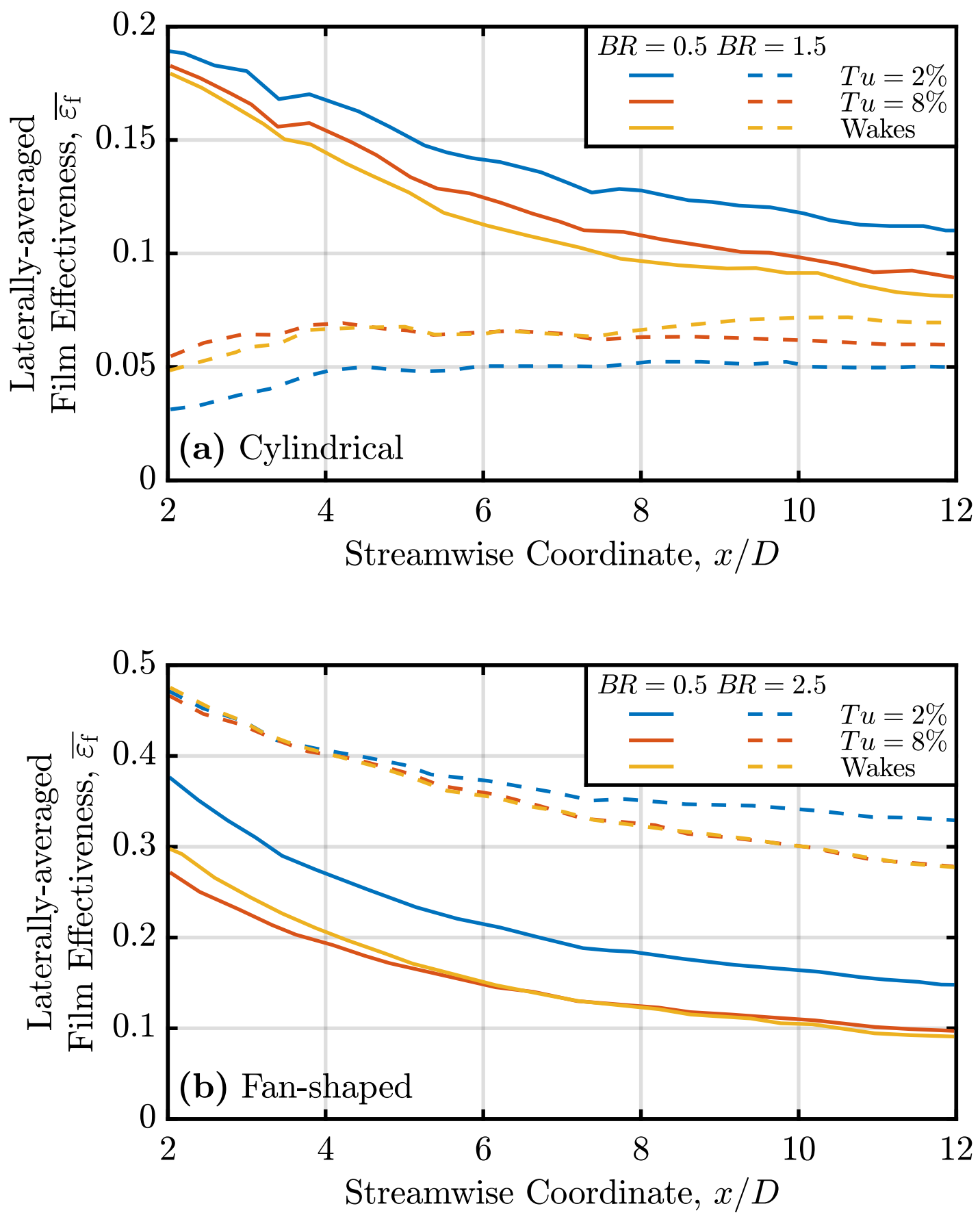

Fig. 2.5 Laterally-averaged film effectiveness data by Saumweber and Schulz (2012), showing effects of steady main-stream turbulence and unsteady wakes on (a) cylindrical holes, and (b) fan-shaped holes. The effects of main-stream turbulence and wakes are qualitatively identical. 
a negligible effect on centreline film effectiveness is observed. At a non-quasi-steady reduced frequency of $\kappa_{\mathrm{c}}=1.4$, the centreline film effectiveness reduces by up to $12 \%$ relative to the steady case. It is asserted that oscillations in coolant trajectory act to spread coolant over a larger volume, reducing effectiveness.

The effect of hole length-to-diameter ratio, $L / D$, was studied by Seo et al. (1999). Short holes with $L / D=1.65$ are most affected by unsteadiness, with the centreline film effectiveness reducing by $54 \%$ at $I R=0.27$ and $\kappa_{\mathrm{c}}=0.25$. Phase-resolved coolant concentration measurements show oscillation of the coolant trajectory in response to main-stream unsteadiness, causing increased spreading in the time-average. However, at a higher momentum flux ratio, $I R=1.1$, pulsations act to increase the centreline film effectiveness by up to $37 \%$. This is explained by the fact that the coolant is instantaneously attached during oscillation, whereas the coolant would always be lifted off in steady state.

\subsection{Computational modelling of film cooling}

Turbine designers require accurate knowledge of the performance of candidate cooling configurations to produce a successful design. Experimental testing is too costly and time-consuming to be used for every design iteration, so testing is supplementary to computational methods and empirical correlations. At present, computational predictions of film cooling are subject to uncertainties, such that the results are not sufficiently robust to eliminate empiricism from design tools (Ireland, 2014).

For some time, Reynolds-averaged Navier-Stokes computations have been the typical approach for turbine design computations. Walters and Leylek (1997) reported RANS simulations of a cylindrical cooling hole, with physically-correct boundary conditions, accurate geometry representation, and adequate spatial discretisation allowing a judgement of turbulence model performance. The simulations show limited agreement with experimental data, with errors of up to $150 \%$ in centreline film effectiveness at a blowing ratio $B R=1.0$. The most accurate predictions are at $B R=0.5$, when coolant is attached to the wall, where the centreline film effectiveness is over-predicted by up to $35 \%$. Other RANS film cooling simulations are reviewed by Bogard and Thole (2006). A common trend is under-prediction of lateral spreading of coolant away from the jet centreline. For example, Walters and Leylek (1997) report an under-prediction of lateral coolant coverage by up to $47 \%$. No RANS turbulence model has been found to give consistent and accurate predictions of film effectiveness (Ferguson et al., 1998; Harrison and Bogard, 2008). 
Since these early simulations, the increase in available computing power has facilitated higher-fidelity simulations, with the possibility of overcoming turbulence model limitations by resolving a greater part of the turbulent energy cascade. The first reported high-fidelity simulation of film cooling was the large-eddy simulation (LES) performed by Tyagi and Acharya (2003). For a cylindrical cooling hole at low blowing ratio, the centreline film effectiveness was predicted to within 10\%, an improvement over RANS. Analysis of the instantaneous flow field shows the existence of coherent vortical structures, with a length scale on the order of the cooling hole diameter, which cannot be resolved in a steady computation and are not amenable to Reynolds-averaged modelling.

A listing of open-literature high-fidelity film cooling simulations is shown in Table 2.1. All LES have near-wall grid spacings $\Delta x \leq 80, \Delta y \approx 1, \Delta z \leq 25$, meeting typical resolution guidelines for wall-resolved LES (Tucker, 2013). A range of algorithms are used, including both second- and higher-order spatial discretisations. Explicit time stepping is most common, but implicit and dual time stepping are also employed. In the former case, time steps are small and set by the size of the smallest mesh element. In the latter cases, the time step can be varied freely to resolve the time scales of interest; the upper bound of reported values is $\Delta t V_{\infty} / D=0.02$, where $\Delta t$ is the time step, $V_{\infty}$ is the main-stream velocity, and $D$ the cooling hole diameter. Ziefle and Kleiser (2013) demonstrated the importance of inflow turbulence: omitting turbulent fluctuations in the upstream boundary layer caused a first-order error in predicted laterally-averaged film effectiveness.

Conventional LES, where the filtered Navier-Stokes equations are solved with an explicit sub-grid scale model, is employed by a narrow majority of workers (Bidan et al., 2013; Fujimoto, 2012; Leedom and Acharya, 2008; Peet and Lele, 2008; Tyagi and Acharya, 2003; Zhong et al., 2016; Ziefle and Kleiser, 2013). Detached-eddy simulation (DES), which operates as URANS in boundary layers and LES away from walls, is used by Kim and Hassan (2010) and Foroutan and Yavuzkurt (2015). Implicit sub-grid scale modelling, where numerical dissipation is assumed to account for unresolved turbulent dissipation, is also used by some authors (Guo et al., 2006; Konopka et al., 2013; Muldoon and Acharya, 2006; Oliver et al., 2017; Renze et al., 2008).

The number of grid points used varies between the computations over about one order of magnitude, from 1 million to 16 million, neglecting the outlier of Muldoon and Acharya (2006) which is an order of magnitude greater still. The confounding factors of different computational codes with different orders of spatial and temporal integration, and different Reynolds numbers, both have a large effect on the mesh resolution required for equivalent accuracy. This prevents the drawing of any precise conclusion on the 


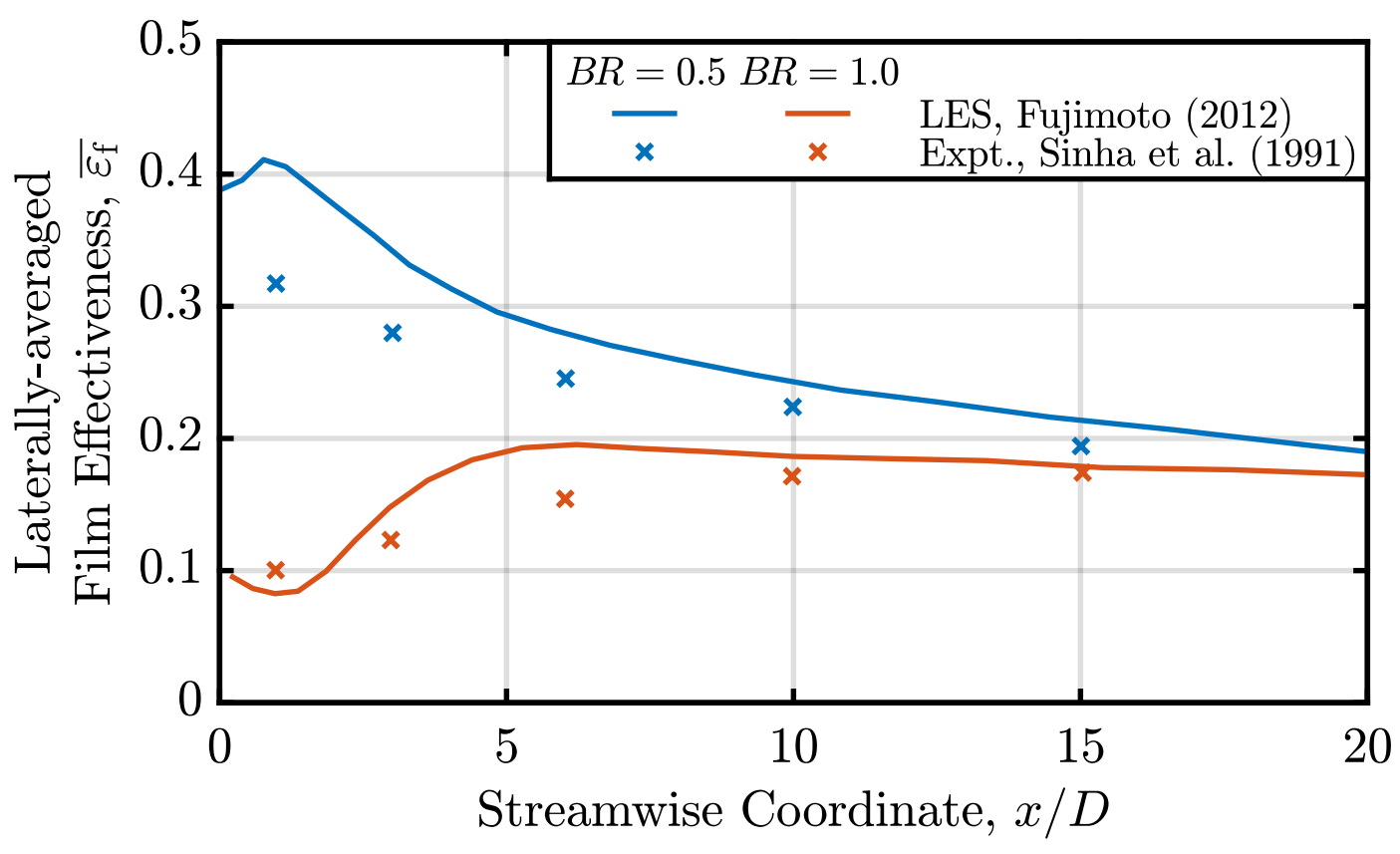

Fig. 2.6 Validation of LES predictions of laterally-averaged film effectiveness by Fujimoto (2012) against experimental data for cylindrical cooling holes from Sinha et al. (1991). The computations are accurate to within $\pm 29 \%$.

required number of grid points for a successful simulation. However, recent studies with second-order codes have used approximately 15 million points (Konopka et al., 2013; Zhong et al., 2016).

The current state of the art is restricted to single cooling holes on flat plates - assuming a conservative 10 million points are required per hole, a single-row calculation of one three-dimensional blade passage with, say, 200 cooling holes would require an impractical 2 billion grid points. Three-dimensional single-stage or multi-stage calculations are therefore not possible with present computing capability.

It is not uncommon to perform no validation of film effectiveness predictions at all. Most studies compare centreline film effectiveness with experimental data. This is inadequate because it does not assess the modelling of lateral coolant spreading, and furthermore the laterally-averaged effectiveness is what is of interest to the designer. Comparisons at a single blowing ratio leave the possibility of 'tuning' the computational method to give good results for a particular case, without generality. Fujimoto (2012) is the only worker to compare values of laterally-averaged film effectiveness at multiple blowing ratios with the same computational model, as shown in Figure 2.6. Reasonable 
agreement is achieved for a cylindrical hole case, to within $\pm 29 \%$. The predictions in Figure 2.6 are representative of the current state of the art. 
Table 2.1 Reported simulations of film cooling using high-fidelity methods, — indicates no information provided, abbreviations CL: centreline; LA: laterally-averaged.

\begin{tabular}{|c|c|c|c|c|c|c|c|}
\hline Reference & $\begin{array}{l}\text { Spatial } \\
\text { order }\end{array}$ & $\begin{array}{c}\text { Mesh } \\
\text { points } / 10^{6}\end{array}$ & $\begin{array}{c}\text { Time step } \\
\Delta t V_{\infty} / D\end{array}$ & $\begin{array}{l}\text { Time step } \\
\text { algorithm }\end{array}$ & $\begin{array}{c}\text { Turbulence } \\
\text { model }\end{array}$ & $\begin{array}{c}\varepsilon_{\mathrm{f}} \\
\text { val'n. }\end{array}$ & $\begin{array}{c}\text { Blowing } \\
\text { ratio }\end{array}$ \\
\hline Tyagi and Acharya (2003) & 4 & 1.1 & 0.001 & explicit & mixed SGS & $\mathrm{CL}$ & 0.5 \\
\hline Muldoon and Acharya (2006) & 4 & 106 & 0.002 & explicit & implicit & - & 0.5 \\
\hline Guo et al. (2006) & 2 & 2.7 & - & explicit & implicit & - & $0.1,0.5$ \\
\hline Renze et al. (2008) & 2 & 5.7 & 0.02 & implicit & implicit & $\mathrm{CL}$ & 0.5 \\
\hline Peet and Lele (2008) & - & 5.5 & 0.0025 & explicit & Smagorinsky & LA & 0.5 \\
\hline Leedom and Acharya (2008) & 3 & 4.2 & - & explicit & Smagorinsky & - & 0.6 \\
\hline Kim and Hassan (2010) & 2 & 1.3 & 0.008 & dual & $k-\epsilon \mathrm{DES}$ & LA & 1.0 \\
\hline Fujimoto (2012) & 2 & 6.2 & 0.001 & implicit & Yoshizawa & $\mathrm{LA}$ & $0.5,1.0$ \\
\hline Ziefle and Kleiser (2013) & 4 & 1.8 & 0.001 & explicit & deconvolution & LA & 0.5 \\
\hline Konopka et al. (2013) & 2 & 15 & 0.0003 & explicit & implicit & - & $0.3,0.6$ \\
\hline Bidan et al. (2013) & 2 & 1.6 & 0.003 & explicit & Smagorinsky & - & various \\
\hline Foroutan and Yavuzkurt (2015) & - & 1.4 & 0.02 & dual & $k-\epsilon \mathrm{DES}$ & $\mathrm{CL}$ & $0.5,1.5$ \\
\hline Zhong et al. (2016) & 2 & 15 & 0.01 & dual & WALE SGS & LA & 0.5 \\
\hline Oliver et al. (2017) & 4 & 16 & 0.0002 & explicit & implicit & CL & 2.0 \\
\hline
\end{tabular}




\subsection{Summary of literature and scope of the thesis}

The implications of the reviewed literature for the design of rotor film cooling are unclear. It is known that the performance of rotor film cooling does not correspond to cascade testing, with the pressure side tending to be more detrimentally affected than the suction side. It has also been demonstrated that main-stream unsteadiness can be detrimental to film effectiveness, by up to $54 \%$, in simplified non-rotating geometries with idealised boundary conditions. The impact of moving-bar wakes is consistent with an increase in time-averaged turbulence intensity. The impact of potential field interaction has been attributed to oscillation of the coolant trajectory. However, in non-rotating studies, the amplitude of main-stream unsteadiness is either not reported, or non-negligible effects on film effectiveness occur only at amplitudes and reduced frequencies exceeding representative values. This hinders direct application of the literature results to design practice.

There is no general description of the way in which unsteadiness alters film cooling performance, applicable to all forms of blade row interaction and cooling hole geometries and configurations. The work in Chapter 5 uses experimental measurements to investigate what makes a particular cooling design susceptible to unsteadiness.

At present, no analysis exists of the mechanisms of blade row interaction as they affect rotor film cooling. In particular, the relative contributions of different mechanisms have not been assessed. This is important because such information would direct the turbine designer towards aerodynamic changes that mitigate the effects of blade row interaction. Chapter 6 addresses this gap using a combination of URANS computations and simplified cooling hole modelling, and provides recommendations for the design of rotor film cooling taking account of blade row interaction.

The computations reviewed in Section 2.5 give some indication of the requirements for a successful film cooling simulation, but do not display progress towards a CFD method that is credible as a design tool. No results are reported applying the same computational model across multiple geometries and operating conditions, which is required to assess the predictive capability of a method. In Chapter 7, film effectiveness predictions using a hybrid URANS-LES approach are validated against experimental data for both cylindrical and fan-shaped holes.

Finally, the data reported in rotating-stage experiments are sparse. A virtual experiment, using the URANS-LES approach from Chapter 7, to quantify the effect of blade row interaction on rotor film effectiveness in a representative turbine cascade is presented in Chapter 8. 



\section{Chapter 3}

\section{Experimental methods}

A new low-speed, flat-plate experimental rig has been developed for measurement of the aerodynamics and heat transfer performance of film cooling holes subject to unsteady boundary conditions. This chapter describes the design of the apparatus, the matching of relevant non-dimensional groups to turbine conditions, the instrumentation and data processing techniques used to characterise the flow field, and finally their associated measurement uncertainties.

\subsection{Rig design and flow similarity}

All experimental work presented in Chapter 5 was performed on the Unsteady Film Cooling Rig (UFC Rig) at the Whittle Laboratory. This a new apparatus designed and built for the current project, to take aerodynamic and heat transfer measurements of film cooling subject to unsteady main-stream boundary conditions representative of blade row interaction in a turbine rotor. Figure 3.1 illustrates the general arrangement of the rig, and the following sections describe the detailed design, and the matching of non-dimensional groups to ensure flow similarity with turbine conditions.

\subsubsection{Rig design}

Main stream The UFC Rig comprises a working section attached to the Bryant blow-down wind tunnel at the Whittle Laboratory, with plenum-fed cooling holes on a flat plate. The main-stream flow is drawn from the atmosphere by a centrifugal blower to a settling chamber, and passes through flow straighteners and a 10:1 contraction into the working section. The working section exhausts to atmosphere. At the exit of the working section is fitted a rotating butterfly valve assembly, driven by a $100 \mathrm{~W}$ inverter 


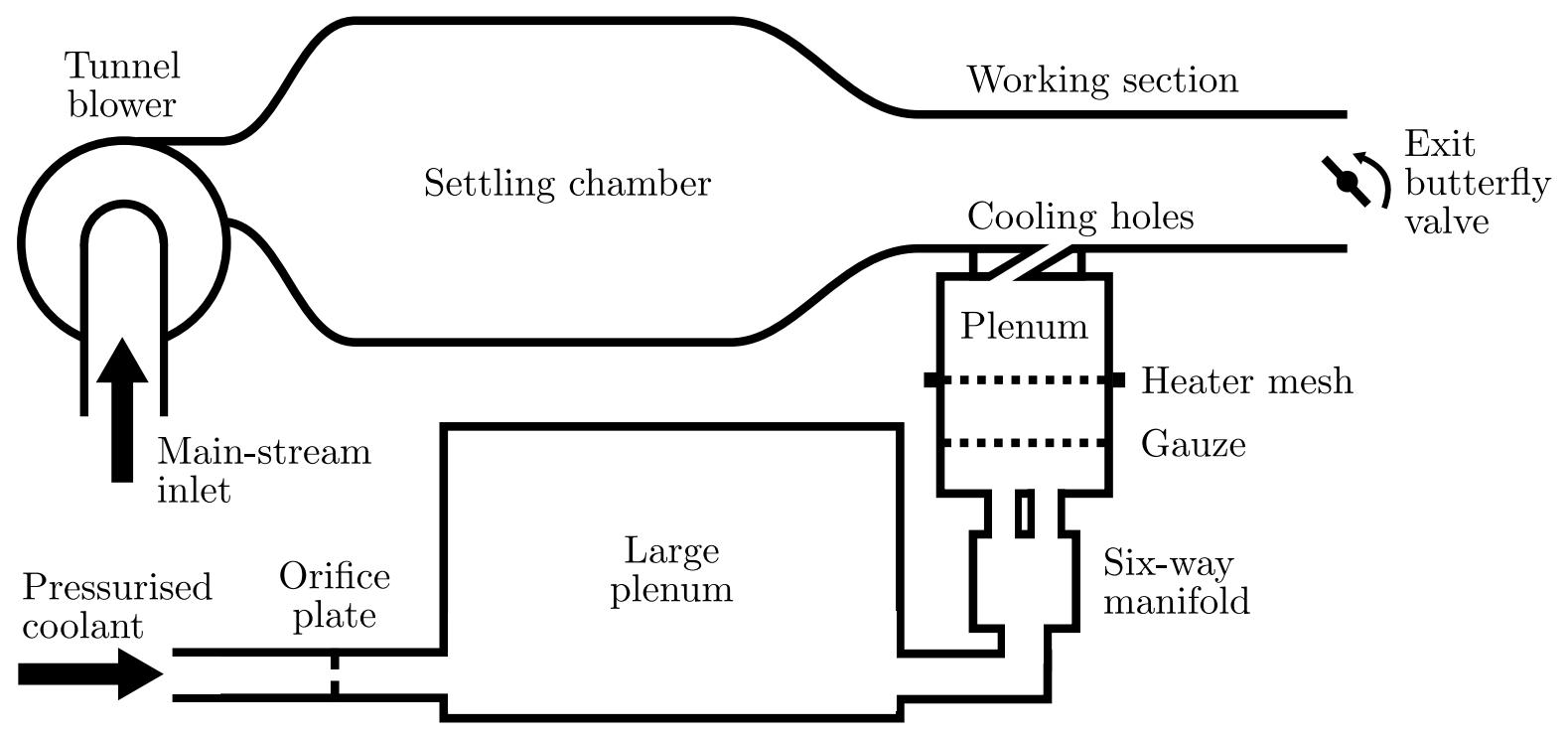

Fig. 3.1 Experimental rig schematic (not to scale).

drive and $\mathrm{AC}$ motor, which by changing the exit area produces an unsteady main-stream static pressure. This is henceforth referred to as the 'pressure fluctuator', intended to model potential field interactions present in a real turbine. An advantage of forcing the cooling holes from downstream in this way is that the time-averaged upstream boundary conditions are independent of the level of unsteadiness.

Coolant Cooling flow is drawn from the atmosphere by a $3 \mathrm{~kW}$ blower before passing through an adjustable bleed valve, an orifice plate mass flow meter, and into a large plenum chamber. This plenum chamber is necessary to decouple mass flow fluctuations through the cooling holes from the blower pressure-rise characteristic. From the large plenum, the coolant flows through a six-way manifold into a smaller plenum chamber, where a heater mesh applies a temperature difference with respect to the main stream. Six inlets are equispaced on the bottom wall of the plenum, which together with a gauze, create a uniform flow field at the heater mesh plane, where any mass flux non-uniformity would create an undesirable temperature non-uniformity. The coolant then enters the cooling holes and joins the main stream. A row of five holes ensures that the middle hole is representative of an infinite, periodic row. The cooling holes are manufactured using rapid prototyping, allowing arbitrary geometry variations within a rectangular block. 
Dimensions Normalising by the cooling hole diameter, $D=20 \mathrm{~mm}$, the dimensions of the working section are $12.5 \mathrm{D}$ in the wall-normal direction, $22.9 \mathrm{D}$ in the lateral direction, and $60 \mathrm{D}$ in the streamwise direction. The row of cooling holes is located $15 \mathrm{D}$ downstream of the working section inlet, and the pressure fluctuator is then $45 D$ downstream of the cooling holes. The plenum chamber has dimensions 23.6D, 22.9D and $12.5 D$ in the wall-normal, lateral and streamwise directions respectively, with the cooling hole inlets located centrally on the top face of the plenum. The rig coordinate system origin is located at the centre of the exit of the middle hole. The streamwise direction is notated $x / D$, the wall-normal direction is notated $y / D$, and the lateral direction is notated $z / D$.

Foam test plate A Rohacell RIMA 110 foam test plate is mounted flush with the tunnel surface downstream of the cooling holes. The streamwise extent of the plate is $4.5 \leq x / D \leq 30$, the lateral extent $-7.5 \leq z / D \leq 7.5$, and the thickness is $1.25 D$. The foam has a low thermal conductivity, $\lambda=0.03 \mathrm{~W} \mathrm{~m}^{-1} \mathrm{~K}^{-2}$, to provide quasi-adiabatic steady-state conditions during heat transfer tests.

\subsubsection{Flow similarity}

There are several non-dimensional groups which should be considered to ensure that the aerodynamic flow field in the experiment is representative of that present in a real turbine. Values of these non-dimensional groups are listed in Table 3.1 for both a turbine rotor film cooling hole and the UFC Rig. Selection and control of the rig values is discussed in the following paragraphs.

Table 3.1 Non-dimensional groups for turbine rotor film cooling and Unsteady Film Cooling Rig. All parameters excluding Mach number and density ratio are matched.

\begin{tabular}{lcc} 
Non-dimensional group & Turbine rotor & UFC Rig \\
\hline Mach number, $M a_{\infty}$ & 0.3 to 0.7 & 0.03 \\
Reynolds number, $R e_{\infty} / 10^{4}$ & 1 to 1.5 & 1.5 \\
Density ratio, $D R$ & 1.3 to 2.0 & 0.9 \\
Momentum flux ratio, $I R$ & 0.5 to 3.0 & 0.2 to 2.0 \\
Reduced frequency, $\kappa_{\infty}$ & 0.005 to 0.01 & 0.005 \\
Pressure fluctuation amplitude, $\Psi$ & 0.02 to 1.0 & $0.0,0.4$ \\
\hline
\end{tabular}


Mach number The flow in the rotor of a large industrial gas turbine is transonic. For the reference turbine used in this work, at cooling hole locations the main-stream Mach number is between $0.3 \leq M a_{\infty} \leq 0.7$. However, conducting experiments at transonic speeds is challenging: compression power requirements scale with Mach number squared, larger pressure forces complicate the mechanical design, and characteristic time scales reduce requiring greater temporal resolution for unsteady measurements. Previous studies (Baldauf and Scheurlen, 1996; Gritsch et al., 1998; Liess, 1975) have shown that while the main stream remains subsonic, the effect of main-stream Mach number on film effectiveness is negligible. Therefore, experiments investigating the effect of main-stream unsteadiness can be conducted in a low-speed facility without loss of applicability.

Reynolds number The Reynolds number should be closely matched as it is the governing parameter for turbulence in the flow. A definition based on hole diameter and main-stream velocity is used,

$$
\operatorname{Re}_{\infty}\left(\rho_{\infty}, V_{\infty}, \mu_{\infty}, D\right)=\frac{\rho_{\infty} V_{\infty} D}{\mu_{\infty}}
$$

The hole diameter is chosen to be $D=20 \mathrm{~mm}$, giving approximately 20 points across the hole using measurement probes of order $1 \mathrm{~mm}$ in size. In this low-speed experiment, the density is fixed at the ambient density. The Reynolds number can then be varied by setting an appropriate velocity via the main-stream blower rotation speed.

Density ratio Coolant conditions are related to main-stream conditions by the blowing, density and momentum flux ratios, Equations (2.2). Fixing two of these and the mainstream conditions sets the coolant conditions. In laboratory experiments, it is necessary to use foreign gases or cryogenics to match the turbine density ratio, which is approximately $1.3 \leq D R \leq 2.0$. With either of these techniques, the allowable mass flow through the cooling holes is limited and hence the hole diameter must be reduced, compromising the spatial resolution of measurement. These complications can be avoided by using a density ratio close to unity, $D R \approx 1$, with a small temperature difference between coolant and main stream as required for heat transfer measurements. This means that only one of the blowing ratio and the momentum flux ratio can be matched to turbine conditions. The coolant momentum determines the trajectory of ejected coolant and liftoff behaviour (Bogard and Thole, 2006), and multiple authors have found in experimental and computational studies that the aerodynamics of cooling holes with different density ratios are comparable at constant momentum flux ratio (Day et al., 2000; Thole et al., 
1992; Walters and Leylek, 2000). Therefore, the experiments match momentum flux ratio to turbine values.

Momentum flux ratio With the main-stream flow already set, the momentum flux ratio can be varied freely via the coolant mass flow rate, using the bleed valve and an inlet throttle on the coolant blower. The momentum flux ratio is defined using an area average,

$$
\operatorname{IR}\left(\dot{m}_{\mathrm{c}}, \rho_{\mathrm{c}}, \rho_{\infty}, V_{\infty}, A_{\mathrm{c}}\right)=\frac{\rho_{\mathrm{c}} V_{\mathrm{c}}^{2}}{\rho_{\infty} V_{\infty}^{2}}=\frac{\left(\dot{m}_{\mathrm{c}} / A_{\mathrm{c}}\right)^{2} / \rho_{\mathrm{c}}}{\rho_{\infty} V_{\infty}^{2}}
$$

Reduced frequency The main-stream reduced frequency is defined,

$$
\kappa_{\infty}=\frac{f D}{V_{\infty}}
$$

which characterises the period of forcing from the unsteady main stream, $1 / f$, relative to a main-stream convective time scale $D / V_{\infty}$. The main-stream reduced frequency is defined in such a way as to be independent of momentum flux ratio. With the hole diameter and main-stream velocity already set by the Reynolds number, the reduced frequency can be matched to turbine conditions by varying the speed of rotation of the pressure fluctuator at the working section exit using the inverter drive.

Pressure fluctuation amplitude The magnitude of unsteady pressure fluctuations in the main stream will affect the response of the cooling hole. Abhari (1996) proposed that the pressure fluctuation should be normalised by the time-averaged coolant dynamic head, however, this leads to a scaling parameter dependent on momentum flux ratio. In the current work, the pressure fluctuation amplitude scaling parameter, $\Psi$, is defined according to,

$$
\Psi\left(\widetilde{p_{\infty}}, p_{0 \infty}-p_{\infty}\right)=\frac{\widetilde{p_{\infty}}}{p_{0 \infty}-p_{\infty}},
$$

where $\widetilde{p_{\infty}}$ is the mean-to-peak fluctuation in main-stream static pressure, and $\left(p_{0 \infty}-p_{\infty}\right)$ is the time-averaged main-stream dynamic head. In the experimental rig, the amplitude can be set by varying the size of the rotating blockage plate of the pressure fluctuator.

\subsection{Aerodynamic measurements}

The instrumentation and measurement techniques used to collect aerodynamic experimental data are described in this section. The instrumentation for determining the 
main-stream operating point is described first, followed by that used for flow-field measurements. Measurement uncertainties are calculated as recommended by Abernethy et al. (1985), with errors propagated using the linearised partial differential method. Bias errors, $B$, and precision errors, $S$, are combined into a total uncertainty to $95 \%$ confidence, $U$, according to,

$$
U=\sqrt{B^{2}+(2 S)^{2}}
$$

\subsubsection{Operating point}

Pressure Ambient pressure, $p_{\mathrm{a}}$, is measured using a digital barometer, model Vaisala PTU 301, accurate to $B\left(p_{\mathrm{a}}\right)= \pm 10 \mathrm{~Pa}$. Precision error in ambient pressure is negligible. Non-time-resolved pressure data are acquired using a Scanivalve DSA 3017 pressure scanner, as gauge pressures, i.e. differentials relative to ambient pressure. Gauge pressures are indicated by a superscript $*$. The pressure scanner has a quoted accuracy of $B\left(p^{*}\right)= \pm 5 \mathrm{~Pa}$; this is thought to be pessimistic as the zero-offset of the sensor is calibrated at the start of each experiment, meaning that error increases from zero as the magnitude of the pressure differential increases. By calculation of the standard deviation of measurements on a port open to atmosphere, the precision error in gauge pressure is found to be $S\left(p^{*}\right)= \pm 0.1 \mathrm{~Pa}$. The main-stream stagnation and static pressures are measured using a Pitot tube and a pressure tapping respectively, placed on a lateral wall of the working section. The static pressure in the coolant plenum, downstream of the heater mesh, is also measured using a set of four pneumatically-averaged tappings on the side walls.

Temperature K-type thermocouples are used to measure the temperatures in the main stream and coolant plenum (downstream of the heater mesh). These temperatures are acquired using a Pico TC08 USB logger. A UKAS-certified calibration suggests that bias errors are approximately $B(T)= \pm 0.5 \mathrm{~K}$, and precision errors are measured at $S(T)= \pm 0.1 \mathrm{~K}$.

Density Although the flow is incompressible, temperature rises through the coolant blower and heater mesh mean that density cannot be assumed uniform at the atmospheric value. Using the ideal gas equation of state allows densities throughout the rig to be calculated according to, for example,

$$
\rho_{\infty}\left(p_{\mathrm{a}}, p_{\infty}^{*}, T_{\infty}\right)=\frac{p_{\infty}^{*}+p_{\mathrm{a}}}{R T_{\infty}}
$$


where $R$ is the specific gas constant. Applying the partial differential method to Equation (3.6), using values measured in a nominal test, the bias and precision uncertainties in density are $B\left(\rho_{\infty}\right)= \pm 0.17 \%$ and $S\left(\rho_{\infty}\right)= \pm 0.03 \%$ respectively.

Velocity In incompressible flow, with density, stagnation and static pressures known, a velocity can be found using Bernoulli's equation,

$$
V_{\infty}\left(\rho_{\infty}, p_{0 \infty}^{*}, p_{\infty}^{*}\right)=\sqrt{2\left(p_{0 \infty}^{*}-p_{\infty}^{*}\right) / \rho_{\infty}}
$$

Because the pressure scanner uses the same sensor to measure both $p_{0 \infty}^{*}$ and $p_{\infty}^{*}$, the bias error in their difference is assumed negligible. Applying the partial differential method to Equation (3.7), using nominal test quantities as before, the bias and precision errors in velocity are $B\left(V_{\infty}\right)= \pm 0.08 \%$ and $S\left(V_{\infty}\right)= \pm 0.09 \%$ respectively.

Coolant mass flow The coolant mass flow, $\dot{m}_{\mathrm{c}}$, is measured using an orifice plate. The configuration consists of a cylindrical pipe interrupted by a constriction or orifice. The pressure drop across the orifice is measured using two sets of four circumferentially-spaced, pneumatically-averaged static pressure tappings. Bias error in this difference is again assumed negligible. A thermocouple is used to measure temperature and hence deduce density upstream of the orifice. The orifice plate is constructed in line with the ISO5167 standard, so no individual calibration is needed and the standard mass flow calculation procedure (a discharge coefficient correlation) is used. Proceeding in accordance with the ISO5167 standard, bias and precision errors are $B\left(\dot{m}_{\mathrm{c}}\right)= \pm 0.88 \%$ and $S\left(\dot{m}_{\mathrm{c}}\right)= \pm 0.19 \%$ respectively.

Viscosity Using Sutherland's Law to determine dynamic viscosity at the main-stream temperature, errors are $B\left(\mu_{\infty}\right)= \pm 0.14 \%$ and $S\left(\mu_{\infty}\right)= \pm 0.03 \%$. 
Non-dimensional operating point All the information required to calculate the non-dimensional operating point of the Rig has now been assembled. The Reynolds number, applying the partial differential method to Equation (3.1), is in error by $B\left(R e_{\infty}\right)= \pm 0.54 \%$ and $S\left(R e_{\infty}\right)= \pm 0.09 \%$, yielding a total root-sum-square uncertainty to $95 \%$ confidence of $U\left(R e_{\infty}\right)= \pm 0.6 \%$. Momentum flux ratio is calculated as an area average according to Equation (3.2). Allowing for a $\pm 0.1 \mathrm{~mm}$ tolerance on the cooling hole diameter $D=20 \mathrm{~mm}$, the bias and precision uncertainties in momentum flux ratio are $B(I R)= \pm 2.7 \%$ and $S(I R)= \pm 0.43 \%$, yielding a total root-sum-square uncertainty to $95 \%$ confidence of $U(I R)= \pm 2.8 \%$ in momentum flux ratio.

Unsteady pressure The pressure fluctuation amplitude is measured using Kulite unsteady pressure transducers, with a frequency response of order $30 \mathrm{kHz}$, much greater than the dimensional forcing frequency of order $f=6 \mathrm{~Hz}$. The transducers are located in the main stream and in the coolant plenum to allow determination of instantaneous hole pressure drop. The main-stream transducer is mounted on the lateral wall of the tunnel, adjacent to the steady static pressure tapping. The plenum transducer is mounted on the lateral wall of the plenum, downstream of the heater mesh. Raw voltage output is amplified, low-pass filtered at $10 \mathrm{kHz}$ in hardware, and digitised at $100 \mathrm{kHz}$ using a USB analogue-to-digital converter, model NI USB-4431. Further filtering is performed as necessary in software. The transducers are calibrated in-situ with reference to coolant and main-stream static pressure data taken under steady conditions. The measurement uncertainty is limited by the pressure scanner accuracy; drift in the Kulite zero offset during each experiment is circumvented by using only the unsteady component of the Kulite data, and adding on a time-averaged value measured using the pressure scanner. Since both the numerator and denominator are a difference in pressures, bias errors in the pressure fluctuation amplitude are neglected, $B(\Psi)=0$. Taking precision errors in the Kulite measurements equal to those of a gauge pressure measurement, and applying the partial differential method to Equation (3.4), the precision error in normalised amplitude is $S(\Psi)= \pm 0.48 \%$ and the total root-sum-square uncertainty to $95 \%$ confidence in normalised pressure fluctuation amplitude is $U(\Psi)= \pm 0.96 \%$.

\subsubsection{Flow field}

Traverse gear Pneumatic or hot-wire probes are mounted on a stepper-motor-controlled traverse gear on top of the working section, with degrees of freedom in the lateral and wall-normal directions. The whole assembly can be manually translated in the streamwise direction to access different traverse planes. 
Pneumatic probe A five-hole pneumatic probe was used to measure flow angles and stagnation and static pressure during commissioning. Flow properties at the pneumatic probe location will be denoted by a subscript pp. The probe, illustrated in Figure 3.2(a), is $0.13 D$ in diameter, stepping up to $0.22 D$ after a length of $2.6 D$. The probe head is $0.09 \mathrm{D}(1.8 \mathrm{~mm})$ in diameter. The probe is calibrated in a free-jet wind tunnel over the yaw interval $-36^{\circ}<\alpha_{\mathrm{pp}}<36^{\circ}$ and the pitch interval $-28^{\circ}<\beta_{\mathrm{pp}}<28^{\circ}$ with steps of $2^{\circ}$ in each direction. The pressures on each hole are used to define calibration coefficients according to the formulation of Dominy and Hodson (1993). A settling time of $1 \mathrm{~s}$ was chosen using the hysteresis method of Grimshaw and Taylor (2016). Verification data collected in the calibration tunnel show that precision error in the measurement process is $S\left(\alpha_{\mathrm{pp}}\right)= \pm 0.3^{\circ}$ in flow angle and $S\left(p_{0 \mathrm{pp}}^{*}-p_{\mathrm{pp}}^{*}\right)= \pm 0.6 \%$ in dynamic head. Bias error in flow angle is set by mechanical considerations: alignment with the jet in the calibration tunnel, and fidelity in transferring that datum to the film cooling rig. Conservatively, this error is estimated at $B\left(\alpha_{\mathrm{pp}}\right)= \pm 1^{\circ}$. In use, the largest contribution to bias error is expected to be due to limitations of the calibration - the measured flow field, with non-uniformities, varying probe Reynolds number, and wall blockage effects, is different to that in the calibration tunnel. It is not possible to quantify this error without cross-calibration measurements. Therefore, bias error in dynamic head is not included in the formal uncertainty analysis. Approximating the density at the probe location using the main-stream density, and propagating the errors in main-stream velocity, the total uncertainties to $95 \%$ confidence are $U\left(V_{\mathrm{pp}} / V_{\infty}\right)= \pm 0.63 \%$ in normalised velocity, and $U\left(\alpha_{\mathrm{pp}}\right)= \pm 1.2^{\circ}$ in flow angle.

Hot-wire probe A hot-wire anemometer probe, shown in Figure 3.2(b), is used to measure time-resolved velocity magnitude data. This is fitted into the traverse gear in place of the pneumatic probe. Hot-wire measurements will be denoted with a subscript hw. Convective heat loss from the wire, measured via the change in sensor voltage drop, is used to infer instantaneous velocity. The low heat capacity of the wire permits fast-response measurements. A cut-off frequency of order $40 \mathrm{kHz}$ was measured using a square-wave response test. The sensor voltage is filtered in hardware and logged at $100 \mathrm{kHz}$ using the NI USB-4431 analogue-to-digital converter. A tachometer monitors the rotating pressure fluctuator, producing an electrical pulse once per revolution. Logging the hot-wire and tachometer voltages simultaneously allows ensemble averaging of the phase-locked velocity field. The hot wire is calibrated in-situ with reference to mainstream velocity data calculated from the main-stream Pitot and static pressures. The hot-wire velocity, $V_{\mathrm{hw}}$, is related to the hot-wire voltage, $E_{\mathrm{hw}}$, according to a calibration 
(a) Pneumatic probe

$(D=20 \mathrm{~mm})$

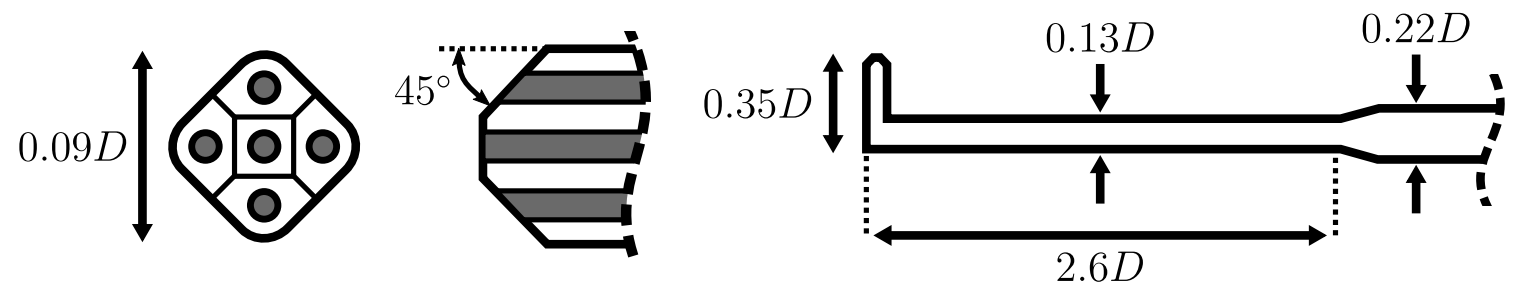

(b) Hot-wire probe
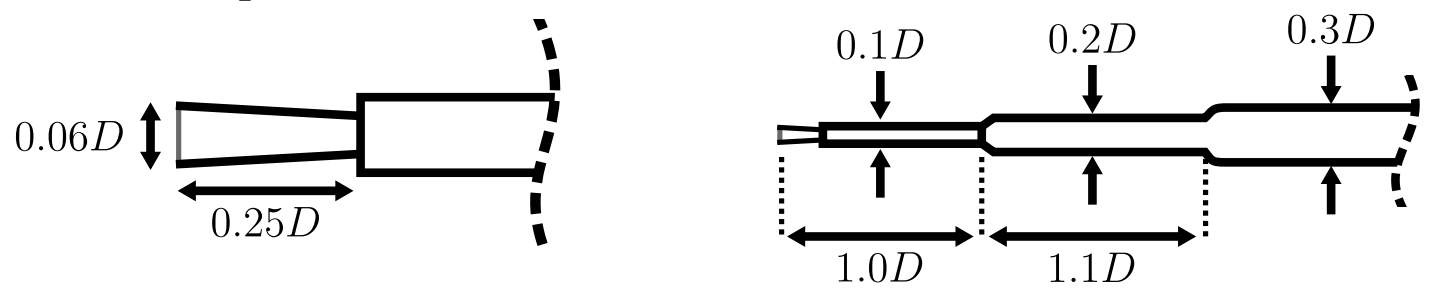

Fig. 3.2 Design of the aerodynamic measurement probes, (a) pneumatic probe, (b) hot-wire probe. Dimensions are referenced to hole diameter $D=20 \mathrm{~mm}$, not to scale.

equation quadratic in log space,

$$
\log \left(V_{\mathrm{hw}}\right)=A \log ^{2}\left(E_{\mathrm{hw}}\right)+B \log \left(E_{\mathrm{hw}}\right)+C,
$$

where $A, B$, and $C$ are constants fitted from calibration data. Back-to-back calibrations show that the measured velocity is repeatable to $S\left(V_{\mathrm{hw}} / V_{\infty}\right)= \pm 0.3 \%$. Bias error arises from ambient temperature variations and calibration drift. The Bearman (1971) correction is used to compensate for variations in ambient temperature, by adjusting the hot-wire voltage to $E_{\mathrm{hw}}^{\prime}$ using,

$$
E_{\mathrm{hw}}^{\prime}=E_{\mathrm{hw}} \sqrt{\frac{\Delta T_{\mathrm{hw}}}{\Delta T_{\mathrm{hw}}-T_{\mathrm{ref}}+T_{\infty}}}
$$

where $\Delta T_{\mathrm{hw}}$ is the hot-wire overheat temperature, $T_{\text {ref }}$ is the main-stream temperature during calibration, and $T_{\infty}$ the current main-stream temperature. Calibration drift is minimised by performing a new calibration before every traverse. After these precautions, the residual bias error may be assessed by considering the variation in measured value of normalised velocity $V_{\mathrm{hw}} / V_{\infty}$ at a main-stream traverse location across all tests, which should be a constant approximately equal to one. The mean value is $V_{\mathrm{hw}} / V_{\infty}=1.01$ with variations of $B\left(V_{\mathrm{hw}} / V_{\infty}\right)= \pm 0.8 \%$. The total uncertainty to $95 \%$ confidence is then $U\left(V_{\mathrm{hw}} / V_{\infty}\right)= \pm 1.0 \%$. 


\subsection{Heat transfer measurements}

In this section, the measurement of heat transfer quantities in the UFC Rig is described. The section begins with an outline of the required background theory, before describing the infra-red thermography and data processing techniques used. Finally, the measurement uncertainty in heat transfer parameters is quantified.

\subsubsection{Characterising cooling performance}

Turbine cooling performance is determined by the rate of convective heat transfer from the main stream into the surface of the cooled component. The convective heat flux, $\dot{q}$, can be modelled using a linear dependence on a driving temperature difference,

$$
\dot{q}=h\left(T_{\mathrm{aw}}-T_{\mathrm{w}}\right)
$$

where $T_{\mathrm{w}}$ is the actual wall temperature, $T_{\mathrm{aw}}$ is the adiabatic wall temperature, and $h$ is the heat transfer coefficient. The adiabatic wall temperature is defined as the wall temperature at which the local heat flux is zero, i.e. the temperature of a perfectly insulating, adiabatic wall. The heat transfer coefficient may be non-dimensionalised as the Nusselt number, $N u=h \ell / \lambda$, where $\ell$ is a characteristic length scale and $\lambda$ is the fluid thermal conductivity. Then, the Nusselt number is a function of the non-dimensional aerodynamic boundary conditions such as Reynolds number and Mach number (plus fluid properties via Prandtl number).

With film cooling, the adiabatic wall temperature at a given location is determined by the degree of mixing between the coolant and the main stream, quantified nondimensionally using the film effectiveness, defined for quasi-adiabatic incompressible flow as in the present experiment,

$$
\varepsilon_{\mathrm{f}}=\frac{T_{\mathrm{aw}}-T_{\infty}}{T_{\mathrm{c}}-T_{\infty}}
$$

where $T_{\infty}$ and $T_{\mathrm{c}}$ are the main-stream and coolant temperatures respectively.

In the real turbine flow, which is non-adiabatic and compressible, the aerodynamic and thermal fields are coupled. This means that, strictly, the heat transfer coefficient and film effectiveness are a function of the upstream wall temperature, i.e. not only of aerodynamic but also of thermal boundary conditions. However, compressibility effects can be accounted for by using the main-stream and coolant recovery temperatures in the definition of effectiveness. At turbine conditions, the main-stream enthalpy flow is high compared to the total heat transfer, so that non-adiabatic effects may be neglected and the main-stream temperature is well-characterised by the upstream recovery temperature. 
That is, the coupling between external aerodynamic and thermal fields is weak. This is not true of the internal coolant, which warms significantly as it flows through the blade. To correct for this, the recovery temperature at the cooling hole exit should be used in the definition of film effectiveness.

When scaling to turbine conditions, both non-adiabatic and compressible effects should be accounted for as in Equation (2.1). Then, to a good approximation, Nusselt number and film effectiveness are indeed a function of aerodynamic boundary conditions only, and may be assumed independent of thermal boundary conditions.

Therefore, to characterise cooling performance, representative aerodynamic boundary conditions are required, while the thermal boundary conditions may be chosen for convenience and to facilitate the most accurate measurements. This means that heat transfer measurements on laboratory experiments that are aerodynamically representative, but quasi-adiabatic, can be scaled to turbine conditions with different, non-adiabatic thermal boundary conditions to predict actual wall temperatures.

For complete non-dimensional similarity, experiments may be conducted with the correct main stream to wall temperature ratio, although matching the turbine value in a laboratory experiment is not straightforward and would cause unnecessary complications for this fundamental study. A comprehensive discussion of scaling heat transfer measurements in cooled compressible flows is given by Luque et al. (2016).

\subsubsection{Infra-red thermography}

A FLIR SC7300LW infra-red camera is used to measure time-resolved distributions of wall temperature on the film-cooled surface, with data acquisition performed using a MATLAB GigE network interface. The camera is mounted above the working section and views the test surface through a Germanium infra-red window. Geometric constraints set by the camera field of view, window size, and rig dimensions mean that the entire film-cooled surface cannot be viewed in a single image. Separate tests are performed with the camera located in three viewing ports at different streamwise locations, which are blanked off when not in use. The processed film effectiveness and heat transfer coefficient measurements are later stitched together to give data over the range $5 \leq x / D \leq 25$ and $-2 \leq z / D \leq 2$.

Calibration of the infra-red camera must be performed to relate captured images to surface temperature distributions. The raw output from the infra-red camera, $\mathcal{I}$, is proportional to irradiance, with a constant of proportionality $k$ dependent on the camera 
optics and detector. The total irradiance may be decomposed into components,

$$
\mathcal{I} / k=\sigma \tau_{\mathrm{w}} \epsilon_{\mathrm{w}} T_{\mathrm{w}}^{4}+\sum_{i} \dot{q}_{\mathrm{err}, i}\left(T_{i}, \epsilon_{i}, \tau_{i}\right)
$$

where $T, \epsilon$ and $\tau$ are the temperature, emissivity, and object-to-camera transmissivity respectively of a radiating body, and $\sigma$ is the Stefan-Boltzmann constant. The first term in Equation (3.12) is the direct irradiance from the test surface of interest, and the second term is the sum of direct and reflected irradiances from other sources that must be corrected for. Error sources include the rig walls, infra-red window, and the atmosphere. The test surface is painted black, with an emissivity $\epsilon_{\mathrm{w}} \approx 0.95$, in order to reduce the magnitude of reflected irradiances.

The error terms, $\dot{q}_{\text {err }, i}$, are functions of the transmissivity and emissivity of each source, which, over a limited temperature range, may be taken as constant. The error terms are also functions of the temperature of each object, which may also be taken as constant over the course of a test. This is because, in the present apparatus, the main-stream temperature is held constant and transient measurements are enabled by applying a step to the coolant temperature. The coolant mass flow is approximately two orders of magnitude lower than the main-stream mass flow, and thus does not alter the temperatures of the rig walls (excluding the film-cooled surface). However, the errors will be a function of the main-stream and atmospheric temperatures. Therefore, Equation (3.12) may be simplified to,

$$
\mathcal{I}=A T_{\mathrm{w}}^{4}+B, \quad \text { with } \quad A=\text { constant }, \quad B=\text { function }\left(T_{\infty}, T_{\mathrm{a}}\right) .
$$

The calibration gradient $A$ is found through fitting to images of a heated metal plate placed in the working section, instrumented with a surface thermocouple, taken as the plate naturally cools down. Before each calibration run, a non-uniformity correction is performed by imaging a quasi-black body at a uniform temperature. Over twenty repeated calibrations, the gradient takes a mean value of $A=1.336 \times 10^{6}$ varying by $\pm 1.7 \%$. In subsequent analysis, the gradient is assumed constant, and uniform over all detector pixels.

The calibration offset $B$ is calculated before each test using an image taken with no coolant flow, $\mathcal{I}_{\infty}$, and a main-stream thermocouple reading, assuming the test plate is adiabatic in steady state and hence $T_{\mathrm{w}}=T_{\infty}$ at this condition. The value of $B$ is then,

$$
B=\mathcal{I}_{\infty}-A T_{\infty}^{4}
$$


Setting the offset in this way means that uncertainty in wall temperature increases with departure from the main-stream temperature, i.e. with local film effectiveness. The method is robust to errors in calibration gradient. Taking a nominal $T_{\infty}=293 \mathrm{~K}$ and a coolant to main-stream temperature difference of $40 \mathrm{~K}$, after some manipulation of Equations (3.13) and (3.14), the worst-case error in $T_{\mathrm{w}}$ due to the measured calibration gradient repeatability of $\pm 1.7 \%$ is $\pm 0.6 \mathrm{~K}$. Calculating the calibration offset using the main-stream thermocouple also has the advantage that, neglecting second-order effects, bias errors in the main-stream thermocouple do not propagate to the numerator of film effectiveness, $\left(T_{\mathrm{aw}}-T_{\infty}\right)$. Because it is only the difference with respect to the main stream that is used in subsequent heat transfer analysis, the total bias error in calibrated surface temperature measurement may be taken as just $B\left(T_{\mathrm{w}}\right)= \pm 0.6 \mathrm{~K}$. The precision error, from the standard deviation of the measurement of a constant-temperature target is $S\left(T_{\mathrm{w}}\right)= \pm 0.05 \mathrm{~K}$.

\subsubsection{Transient linear regression}

From Equation (3.10), determination of the heat transfer coefficient requires measurement of pairs of heat flux and driving temperature difference at multiple thermal boundary conditions. This may be done in steady state, by imposing a series of known heat fluxes as in Lim et al. (2013), or by imposing a series of known wall temperatures as in Saumweber et al. (2003). The difficulty with these approaches is that the solid-side thermal boundary conditions must be quantified. Lim et al. (2013) used an empirical correction for conductive and radiative heat losses in their calculation of surface heat flux. Saumweber et al. (2003) used a high-conductivity test plate, instrumented with thermocouples on the back side, to provide well-posed boundary conditions for a threedimensional solid conduction computational model, which was used to calculate surface heat flux.

In transient measurement techniques, a rapid change in fluid temperature is imposed, altering the driving temperature difference, and heat transfer quantities are deduced from the dynamic response of the surface temperature. In the UFC Rig, a step change is introduced to the coolant temperature using the heater mesh located in the plenum. The advantage of transient methods is that, for sufficiently short test times, the solidside boundary conditions are semi-infinite and well-posed; no further measurements are required to calculate the surface heat flux. The semi-infinite assumption is valid while,

$$
t \alpha_{\mathrm{w}} / \ell^{2} \ll 1
$$




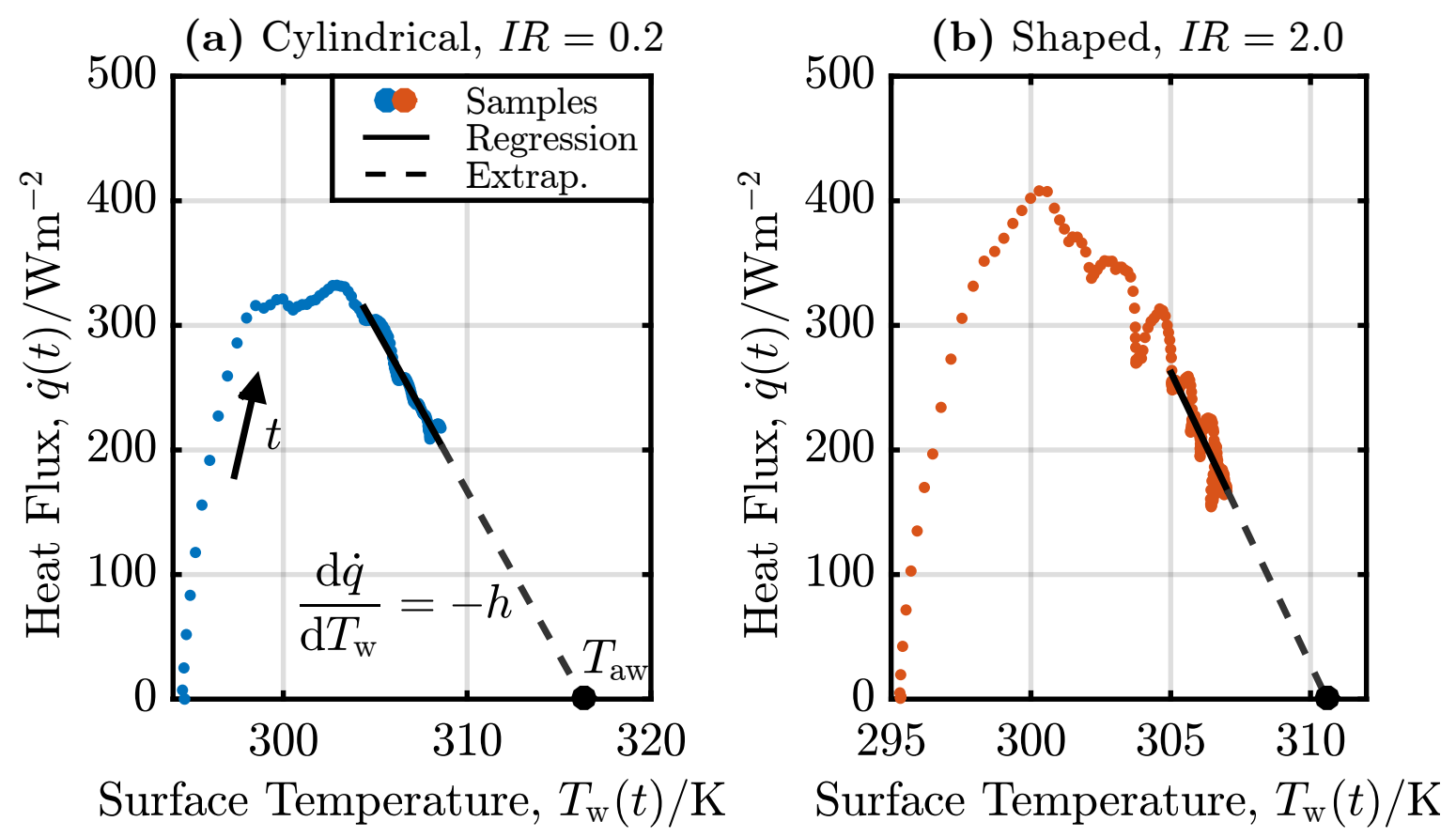

Fig. 3.3 Example transient linear regression data of instantaneous surface temperature and heat flux: (a) cylindrical hole, $I R=0.2$; $(\mathbf{b})$ shaped hole, $I R=2.0$.

where $t$ is the time since initiation of the transient, $\alpha_{\mathrm{w}}$ is the thermal diffusivity of the test surface, and $\ell$ is the thickness of the test surface. For transient measurements in this thesis, $t \alpha_{\mathrm{w}} / \ell^{2} \leq 0.01$ at all times. Here, making the semi-infinite assumption, the impulse response method of Oldfield (2008) is used to calculate instantaneous surface heat flux, $\dot{q}(t)$, from an instantaneous wall temperature history, $T_{\mathrm{w}}(t)$, measured using the infra-red camera.

Heat transfer parameters may be extracted from the time series data using linear regression, as described by O'Dowd et al. (2011). Equation (3.10) applies at all instants in time, so for a given wall location, a linear relationship is expected plotting $\dot{q}(t)$ against $T_{\mathrm{w}}(t)$. Performing linear regression on this data, the fitted gradient is $-h$, and the horizontal intercept $T_{\mathrm{aw}}$, as illustrated in Figure 3.3(a). The adiabatic wall temperature may then be non-dimensionalised to film effectiveness using Equation (3.11), and the heat transfer coefficient to Nusselt number.

Typical measured data are shown in Figure 3.3. After a starting transient as the temperature step occurs, linear behaviour is observed. The quality of regression fit depends on the level of flow unsteadiness as shown by comparing the two tests of different geometries in Figure 3.3. It is hypothesised that at high momentum flux ratio, a separation within the diffusing shaped hole sheds large-scale turbulent flow structures. 
This leads to greater oscillations about the linear fit in Figure 3.3(b), compared to a cylindrical hole at low momentum flux ratio in Figure 3.3(a).

\subsubsection{Heat transfer measurement uncertainty}

The uncertainty in measured film effectiveness and heat transfer coefficient are now considered. A complete a-priori error analysis for the heat transfer measurement and data processing chain is not amenable to the linearised partial differential method, and a more complex analysis is outside the scope of this work; the problem has been considered at length by Playford (2018). Instead, it is more straightforward to estimate measurement uncertainties a-posteriori from real data.

\section{Bias error}

Bias error may be estimated via comparison with an independent measurement method. Laterally-averaged effectiveness data from both the current UFC Rig and the experiment of Saumweber et al. (2003) from the University of Karlsruhe are compared in Figure 3.4. The hole geometries are identical, but the boundary conditions are not. The present rig is quasi-isothermal, whereas the Karlsruhe rig has a turbine-representative density ratio. As justified in Section 3.1.2, the data are compared at constant momentum flux ratio. The Karlsruhe rig is transonic, and takes a main-stream Reynolds number $66 \%$ greater than the UFC Rig. Baldauf and Scheurlen (1996) argued that film effectiveness was independent of both Mach and Reynolds number over ranges representative of turbine conditions. The main-stream turbulence intensity in the Karlsruhe rig is $T u_{\infty}=3.6 \%$, compared to $T u_{\infty}=1 \%$ in the UFC rig. Extrapolating the the Karlsruhe data to $T u_{\infty}=1 \%$, a discrepancy in film effectiveness of order 0.014 is expected. Notwithstanding these differences in flow conditions, agreement between the data sets would increase confidence in the measurement method.

Figures 3.4(a) and 3.4(b) show film effectiveness data for cylindrical and laid-back fan-shaped holes respectively. The shaded bands denote measurement uncertainties or confidence intervals for each data set. For the cylindrical hole, Figure 3.4(a), the data at $I R \approx 0.2$ agree to within the experimental uncertainties. The literature data at $I R=1.3$ is bracketed by data from the present rig at $I R=1.0$ and $I R=2.0$ for $x / D \leq 14$. However, for $x / D>14$, the literature film effectiveness at $I R=1.3$ is greater than for both $I R=1.0$ and $I R=2.0$ in the present rig. This is because, far downstream of the hole, film effectiveness scales with blowing ratio and not momentum flux ratio (Bogard and Thole, 2006). 


\begin{tabular}{|cc|}
\hline UFC Rig & \\
$\square$ & $I R=0.2$ \\
& $I R=0.6$ \\
& $I R=1.0$ \\
& $I R=2.0$ \\
\hline
\end{tabular}

\begin{tabular}{|cc|}
\hline \multicolumn{2}{|l|}{ Saumweber } \\
et al. (2003) \\
-- & $I R=0.15$ \\
--- & $I R=1.32$ \\
$\cdots \cdots$ & $I R=3.68$ \\
\hline
\end{tabular}
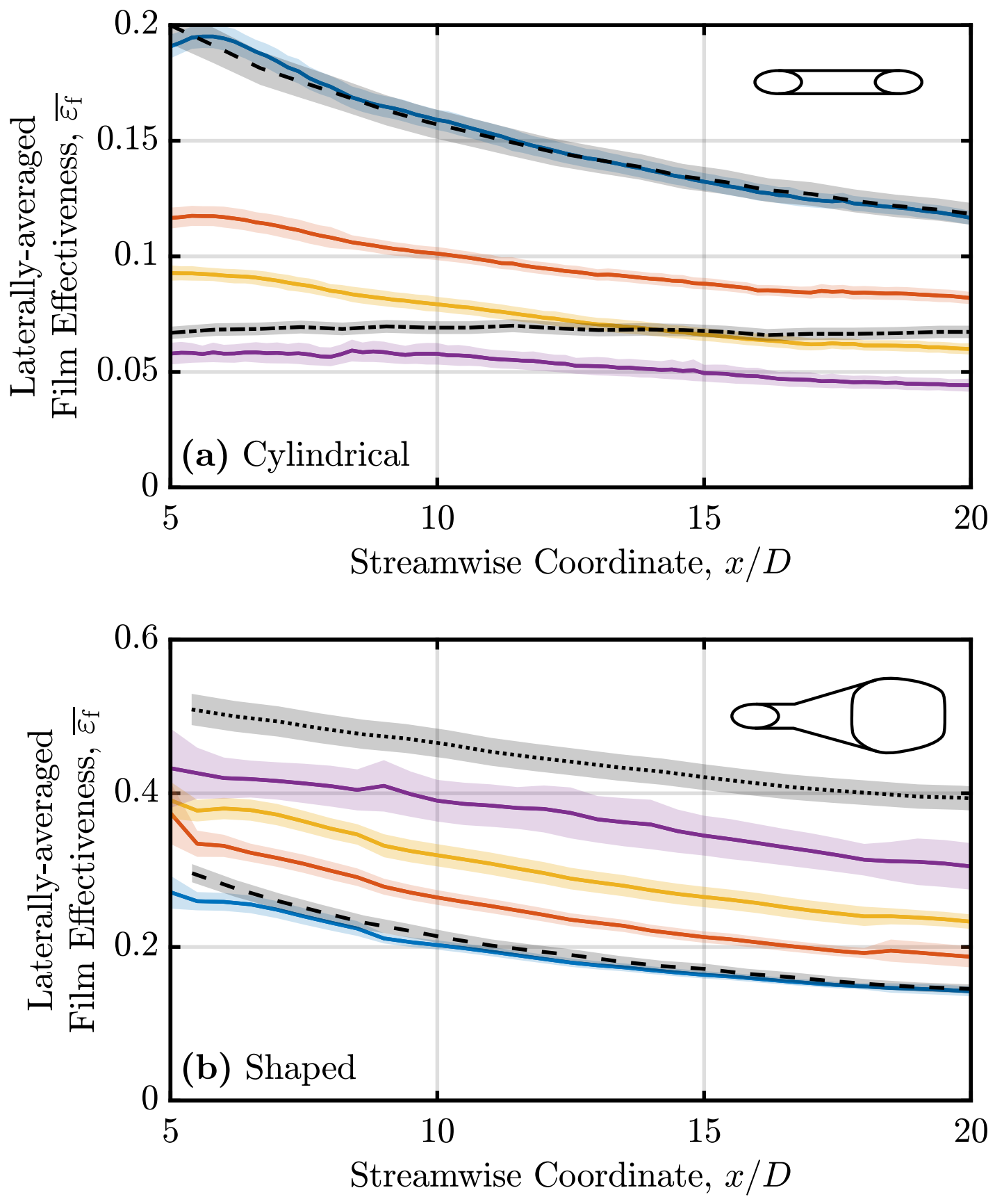

Fig. 3.4 Comparison of film effectiveness distributions measured in the present Unsteady Film Cooling Rig and reported by Saumweber et al. (2003), for (a) cylindrical holes, (b) laid-back fan-shaped holes. 
For the shaped hole, Figure 3.4(b), the data at $I R \approx 0.2$ agree to within the experimental uncertainty. Literature data at high momentum flux ratio are only available for $I R=3.7$, which produces a film effectiveness distribution approximately 0.1 above the $I R=2.0$ data measured in the present apparatus.

Overall, the film effectiveness data from the UFC Rig are consistent with those reported for the University of Karlsruhe experiment. The primary results of the experiment are the changes in film effectiveness due to main-stream unsteadiness, where direct comparisons using the same apparatus and measurement techniques reduce sensitivity to bias errors. Bias errors in film effectiveness are therefore concluded to be acceptable for the purposes of the present investigation.

\section{Precision error}

The single-point precision error, $S$, is calculated as the standard deviation over 20 repeated measurements after discarding two outliers. A $95 \%$ confidence interval for the true mean film effectiveness or heat transfer coefficient, $C I$, is calculated from the repeated measurements using,

$$
C I=S \times \frac{\mathcal{T}(N-1)}{\sqrt{N}}
$$

where $\mathcal{T}(\nu)$ is the Student- $t$ multiplier for $95 \%$ confidence with $\nu$ degrees of freedom, and $N$ is the number of repeats after outliers have been discarded.

Measured values of $S$ and $C I$ in a nominal cylindrical hole test at $I R=0.6$ are shown for film effectiveness in Figure 3.5 and for heat transfer coefficient in Figure 3.6. A line contour of $\varepsilon_{\mathrm{f}}=0.1$ is used to demarcate the region of coolant coverage.

Considering first film effectiveness, in Figure 3.5(a), precision error is up to $S\left(\varepsilon_{\mathrm{f}}\right)=0.026$ at peak effectiveness (on the centreline for $x / D \approx 6$ ) and decreases to $S\left(\varepsilon_{\mathrm{f}}\right) \leq 0.005$ outside coolant coverage. This is because as local film effectiveness and hence local temperature step reduces, the range of heat flux over which $T_{\text {aw }}$ must be extrapolated reduces. At $\varepsilon_{\mathrm{f}}=0$, with no local temperature step, $T_{\mathrm{w}}=T_{\mathrm{aw}}$ throughout the transient. Repeat averaging reduces the uncertainty to a confidence interval of $C I\left(\varepsilon_{\mathrm{f}}\right) \leq 0.013$, Figure 3.5(b). There are steps at $x / D=8$ and $x / D=17$, where the data from multiple camera locations are stitched together, because the confidence intervals are calculated over different sets of repeats. This does not affect the film effectiveness results. While $N=18$ is enough repeats to converge the sample mean, the sample standard deviation would require more repeats to converge to the true value. 
Film Effectiveness Uncertainty

$$
\varepsilon_{\mathrm{f}}=0.1
$$
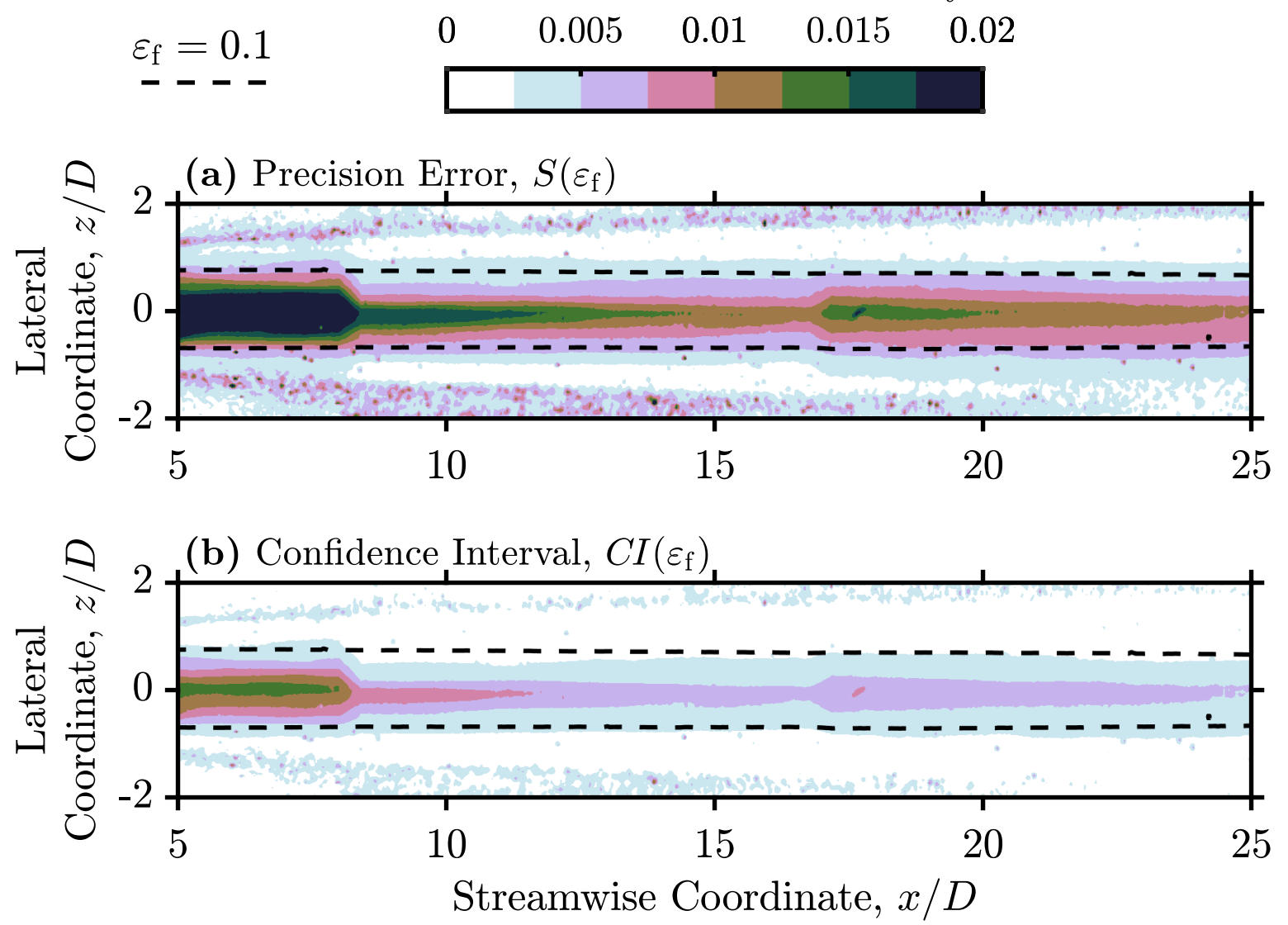

Fig. 3.5 Film effectiveness measurement uncertainty, (a) precision error, and (b) confidence interval, for cylindrical holes at $I R=0.6$ under steady main-stream conditions. 
Heat Transfer Coefficient Uncertainty $/ \%$
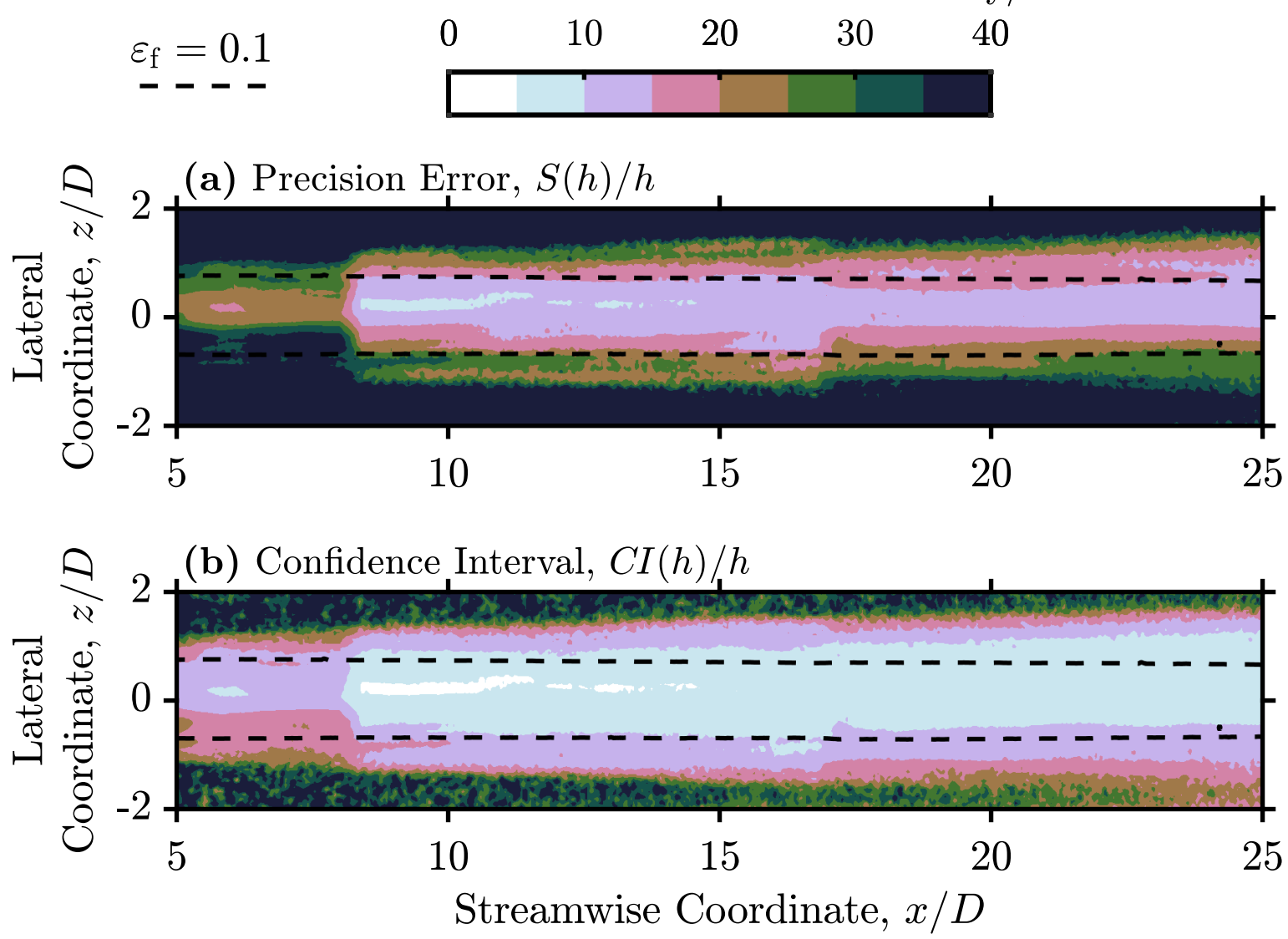

Fig. 3.6 Heat transfer coefficient measurement uncertainty, (a) precision error, and (b) confidence interval, for cylindrical holes at $I R=0.6$ under steady main-stream conditions. 
Relative precision error in heat transfer coefficient is shown in Figure 3.6(a). Within coolant coverage, values range from $S(h) / h \approx 10 \%$ to up to $S(h) / h \approx 40 \%$. The larger errors for $x / D \leq 8$ are caused by an additional outlying repeat at that camera position which has not been discarded. Outside coolant coverage, where the temperature step is small, regression cannot be performed to extract the heat transfer coefficient and first-order precision errors are calculated. Repeated averaging reduces the confidence interval to $C I(h) / h \leq 20 \%$ for $\varepsilon_{\mathrm{f}} \geq 0.1$, Figure 3.6(b). Errors in length scale and thermal conductivity are much smaller than these errors in heat transfer coefficient, so are neglected and $C I(h) / h=C I(N u) / N u$.

During the experimental campaign, it was found that this level of uncertainty was too high to draw any definitive conclusions from the heat transfer coefficient data, and because the scope of this work is restricted to film effectiveness (as discussed in Section 2.1), heat transfer coefficients are not discussed in the remainder of the thesis.

The primary output of interest from the experiment is film effectiveness reduced into streamwise distributions by area-averaging across the hole pitch to form the laterallyaveraged film effectiveness, $\overline{\varepsilon_{\mathrm{f}}}$. If errors are perfectly correlated across the hole pitch (a conservative assumption), the confidence interval is equal to the laterally-averaged confidence interval. As discussed in the above sections, the uncertainty in film effectiveness is a function of both the heat flux extrapolation range and flow unsteadiness. Separate confidence intervals are calculated for each test and displayed with the laterally-averaged film effectiveness data where it is presented in Chapter 5. For cylindrical holes under steady boundary conditions, $C I\left(\overline{\varepsilon_{\mathrm{f}}}\right) \leq 0.005$. With unsteady boundary conditions, the confidence interval rises to $C I\left(\overline{\varepsilon_{\mathrm{f}}}\right) \leq 0.01$. Higher values of film effectiveness and flow unsteadiness lead to greater uncertainties of $C I\left(\overline{\varepsilon_{\mathrm{f}}}\right) \leq 0.04$ for the shaped hole cases.

\subsection{Summary of measurement uncertainties}

Table 3.2 lists the measurement uncertainties for the quantities measured in the Unsteady Film Cooling Rig. The non-dimensional operating point groups are all known to within $2.8 \%$. Flow field measurements are accurate to within $1.0 \%$ normalised velocity and $1.2^{\circ}$ for flow angle. Comparison to literature data suggests that bias errors in film effectiveness are acceptably low for the back-to-back comparisons made in this work. Precision error in laterally-averaged film effectiveness varies with each case; after averaging confidence intervals range from 0.005 for cylindrical holes under steady boundary conditions, to 0.04 for shaped holes at high momentum flux ratio. Heat transfer coefficient data are only 
available where there is coolant coverage, with a relative uncertainty of approximately $20 \%$. 
Table 3.2 Measurement uncertainties in the Unsteady Film Cooling Rig. Values in parentheses denote averaged confidence intervals, halved to be consistent with the definition of $S$ in Abernethy et al. (1985).

\begin{tabular}{llll} 
& Bias, $B$ & Precision, $S$ & Total, $U$ \\
\hline \hline Operating point & & & \\
\hline Reynolds Number, $R e_{\infty}$ & $0.5 \%$ & $0.1 \%$ & $0.6 \%$ \\
Momentum Flux Ratio, $I R$ & $2.7 \%$ & $0.4 \%$ & $2.8 \%$ \\
Pressure Fluctuation Amplitude, $\Psi$ & - & $0.5 \%$ & $1.0 \%$ \\
Flow field & & & \\
\hline Pneumatic Probe Flow Angle, $\alpha_{\mathrm{pp}}$ & $1^{\circ}$ & $0.3^{\circ}$ & $1.2^{\circ}$ \\
Pneumatic Probe Velocity, $V_{\mathrm{pp}} / V_{\infty}$ & - & $0.3 \%$ & $0.6 \%$ \\
Hot-wire Probe Velocity, $V_{\mathrm{hw}} / V_{\infty}$ & $0.9 \%$ & $0.3 \%$ & $1.0 \%$ \\
Heat transfer & & & \\
\hline Local Film Effectiveness, $\varepsilon_{\mathrm{f}}$ & - & $0.026(0.007)$ & 0.013 \\
Nusselt Number, $N u\left(\right.$ where $\left.\varepsilon_{\mathrm{f}} \geq 0.1\right)$ & - & $40 \%(10 \%)$ & $20 \%$ \\
Laterally-averaged Film Effectiveness, $\overline{\varepsilon_{\mathrm{f}}}$ & - & $(0.002$ to 0.02$)$ & 0.005 to 0.04 \\
\hline
\end{tabular}





\section{Chapter 4}

\section{Computational methods}

In the present work, computational fluid dynamics is employed in both low-fidelity simulations of blade row interaction, where cooling holes are treated using a simplified model, and high-fidelity simulations, with resolved cooling holes. This Chapter first describes the turbostream flow solver used. The mesh generation, computation procedure, and post-processing techniques are then presented, for both low-fidelity and high-fidelity simulations.

\subsection{Flow solver}

The flow solver used in the current work is TURBOSTREAm 3.6.4. TURBOSTREAm is based on the TBLOCK algorithms developed by Denton (1992), and was implemented for parallel operation on graphical processing units by Brandvik and Pullan (2011). Full details of the TBLOCK algorithms are given by Klostermeier (2008), and a general overview is given below.

The spatial discretisation is centred, second-order accurate finite-volume on multiblock structured grids. A single-step explicit time marching method is used (Denton, 2002), with local time steps for each cell based on a constant Courant-Friedrichs-Lewy number, the cell size and local acoustic speed. To stabilise the solution, an artificial dissipation term is formed from a blend of second and fourth derivatives of the primary flow variables, with an adaptive coefficient based on pressure and temperature gradients which activates the second-derivative component. In an unsteady computation, temporal accuracy is recovered using dual time stepping, which uses a second-order backward discretisation of the time derivative to form an implicit unsteady source term, after Jameson (1991). Multigrid and negative feedback (Denton, 2017) are used to accelerate the 
convergence of steady and URANS computations only in this thesis, as these techniques were found to cause problems with DES computations.

In unsteady computations, relative motion between stationary and rotating rows is accounted for using a 'sliding plane', which performs a one-dimensional circumferential interpolation procedure to match the flow field at the row interfaces at each time step. Wall shear stress is obtained from adaptive wall functions, requiring that the first grid point away from solid walls is either in the laminar sublayer or the log-law region of the boundary layer. The turbulence model can be selected from: the Spalart and Allmaras (1992) model, a single-equation eddy viscosity method; or the mixing-length model (Brandvik and Pullan, 2011), a simpler algebraic method. Boundary layers are assumed fully-turbulent throughout the domain, and all surfaces are taken as adiabatic. Perfect gas properties are assumed for all computations.

The same solver is used for both low-fidelity, non-film-resolved URANS simulations, and high-fidelity, film-resolved DES simulations. The specific configuration in each of these cases is now described.

\subsection{Non-film-resolved simulations}

The objective of the low-fidelity computations is to predict unsteady main-stream boundary conditions imposed on rotor cooling holes due to blade row interaction. This does not require detailed resolution of the cooling holes themselves, which would entail additional computational expense (about two orders of magnitude). A URANS approach is used with the Spalart-Allmaras turbulence model. The following paragraphs describe the set-up and execution of these computations.

Case The case selected for analysis is representative of a large industrial gas turbine. The computational domain includes the first one-and-a-half stages of the high-pressure turbine, i.e. an upstream stator, rotor, and downstream stator. The upstream vane exit flow is subsonic, without trailing-edge shocks, with a mid-span isentropic Mach number $M a_{\text {ref }}=0.70$. The upstream vane Reynolds number based on mid-span axial chord and isentropic exit velocity is $R e_{\text {ref }}=3.8 \times 10^{6}$, a high value, supporting the assumption of fully-turbulent boundary layers.

Boundary conditions At the inlet plane, experimentally-measured radial profiles of stagnation temperature, stagnation pressure and flow angles are imposed. An exit static pressure distribution is derived from an experimentally-measured hub static pressure 


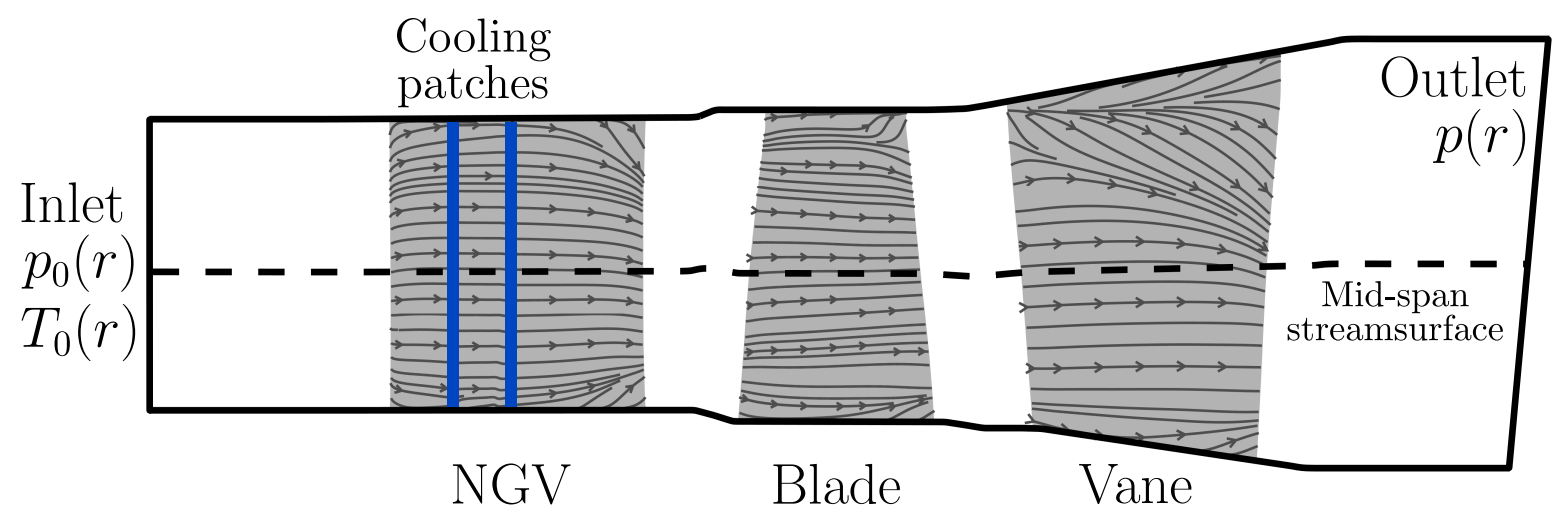

Fig. 4.1 Meridional schematic of three-dimensional turbine case, showing domain, boundary conditions and mid-span streamsurface, with projected suction-side streamlines from RANS computation.

and simple radial equilibrium. The inlet and exit boundaries enforce circumferential uniformity, so to prevent non-physical influence, they are both placed 6.25 vane axial chords away from the stage, with an inviscid hub and casing to prevent boundary layer growth in the inlet section. A representative cooling scheme for all rows is modelled using patches with additional surface fluxes of mass, momentum and enthalpy. This model is not expected to give realistic surface temperature distributions, but captures the effects of coolant on the turbine aerodynamics. Coolant is ejected from the vane, blade and endwall surfaces. A meridional schematic of the domain and boundary conditions is shown in Figure 4.1. The log-law wall function is utilised, with a typical non-dimensional wall resolution of $30 \leq \Delta y^{+} \leq 200$. A one-eighth sector of the annulus is simulated to allow the correct ratios of the upstream vane, blade, and downstream vane counts. 72 time-accurate outer steps are used per rotor passing period.

Mesh A multi-block structured mesh was generated for each of the three rows using the commercial software AUTOGRID5 (Numeca International, 2014). A C-mesh block is placed around the aerofoil surfaces, a 'butterfly' composed of one O- and one H-mesh block is used in the tip gap, and H-mesh blocks are used elsewhere. The mesh is elliptically smoothed on each blade-to-blade streamsurface, with an expansion ratio of 1.2 at solid walls. The mesh has 89 spanwise points with 17 in the rotor tip gap and an overall density of approximately 1.5 million nodes per row. The single-passage meshes are duplicated in the circumferential direction to give a one-eighth annulus sector with 38 million nodes in total. The block topology on a mid-span blade-to-blade surface is shown in Figure 4.2 and the detail of the rotor passage mesh in Figure 4.3. 


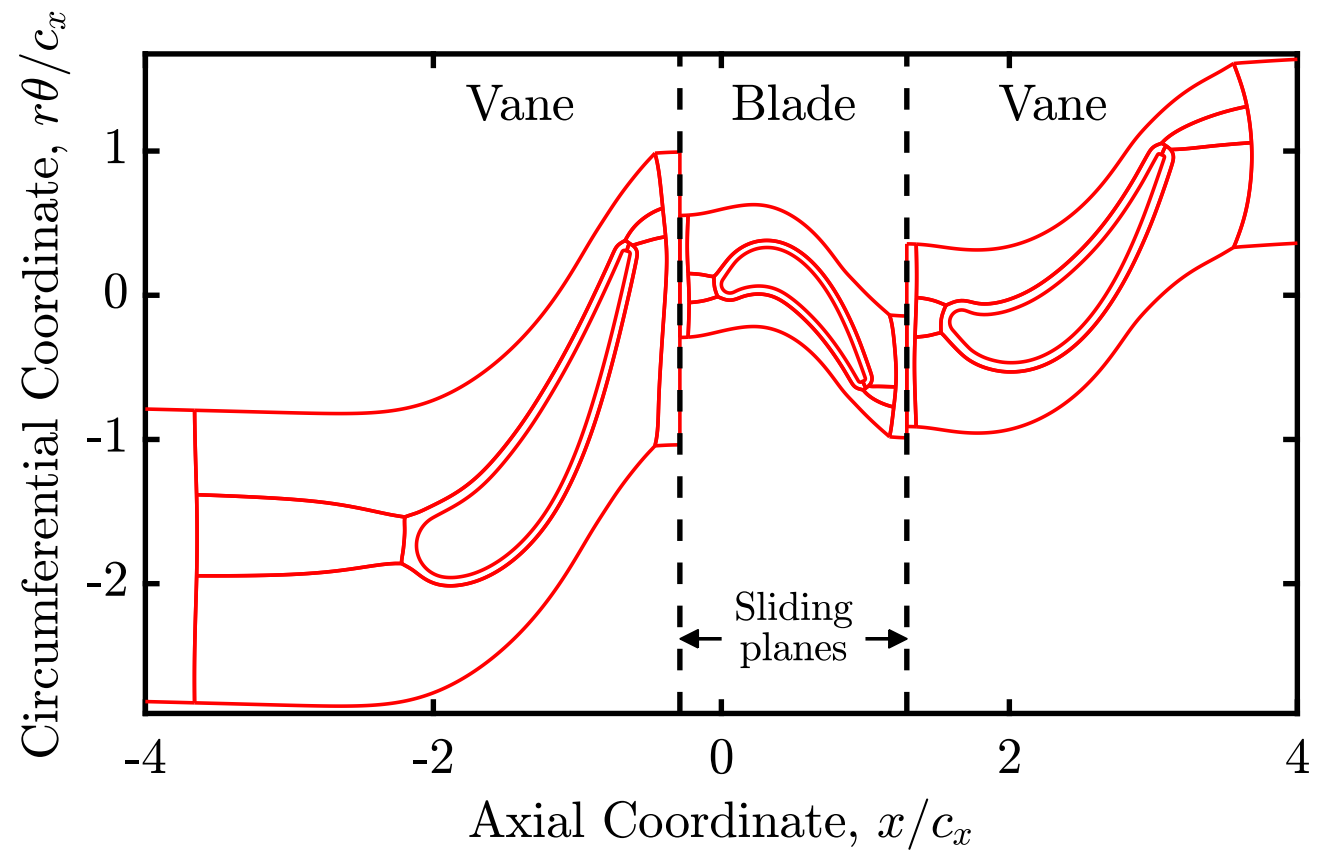

Fig. 4.2 Mid-span block topology of mesh used for non-film-resolved turbine stage computations.

Computation procedure As described in Chapter 6, cases are simulated with modified upstream vanes, with and without film cooling. This means that the rotor mass flow is less than the design mass flow, where all rows are cooled. To accommodate this, the upstream vane is restaggered to correct for changes in deviation, and the blade and downstream vane are restaggered closed, until the mid-span upstream vane exit Mach number is matched with a fully-cooled solution. Each unsteady simulation is started from a converged steady solution and run for two sector periods to flush transients from the domain. Unsteady data are then output over one further sector period for analysis.

Post-processing Although the computation is fully three-dimensional, all analysis is performed at the mid-span streamsurface. As shown by the suction-side streamlines in Figure 4.1, the flow is two-dimensional at this location, simplifying the problem by removing the effects of secondary flows. The flow is non-dimensionalised with reference to mid-span isentropic exit conditions: the stagnation pressure reference is mass-averaged at row inlet, and the static pressure reference is area-averaged at row exit. These are calculated on planes 0.3 rotor axial chords up and downstream of the rows. 


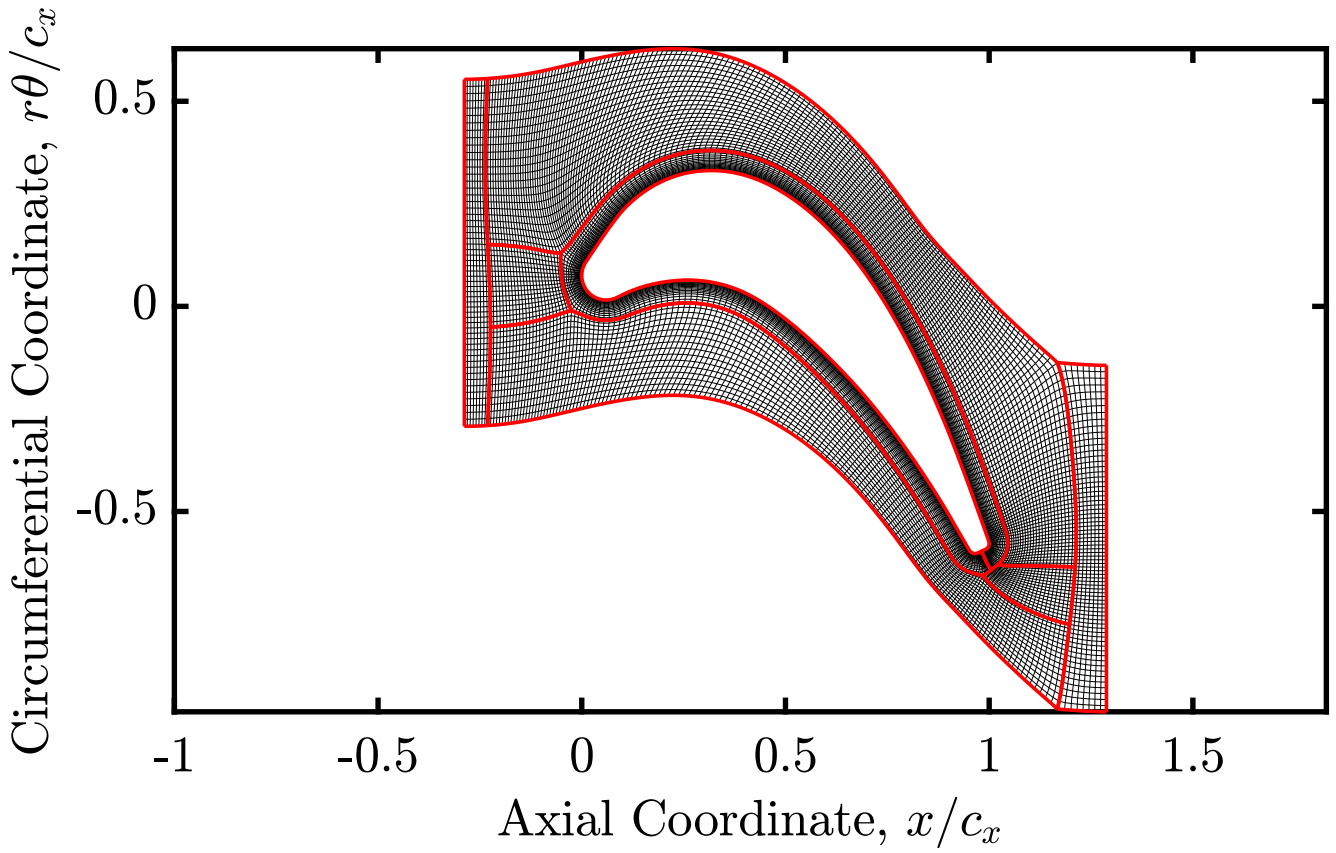

(a) Rotor passage

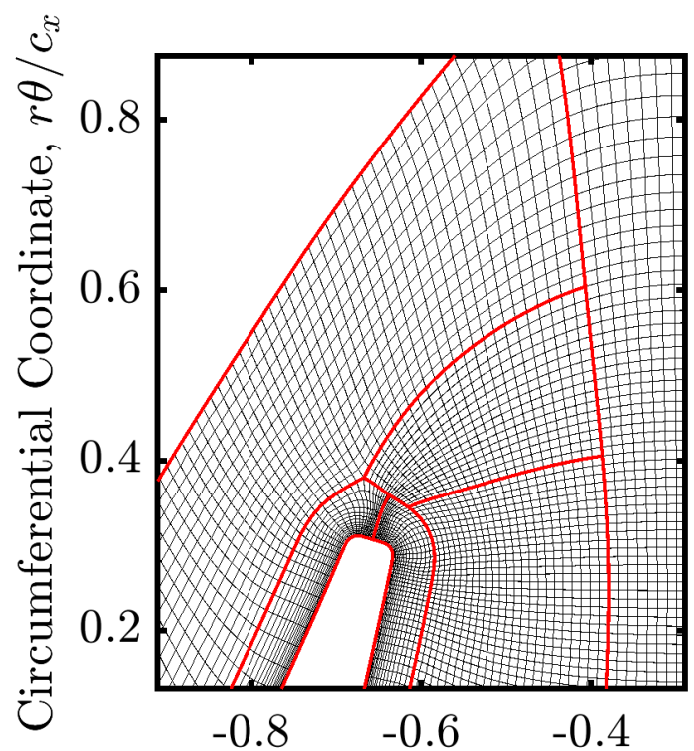

Axial Coordinate, $x / c_{x}$

(b) Upstream vane trailing edge

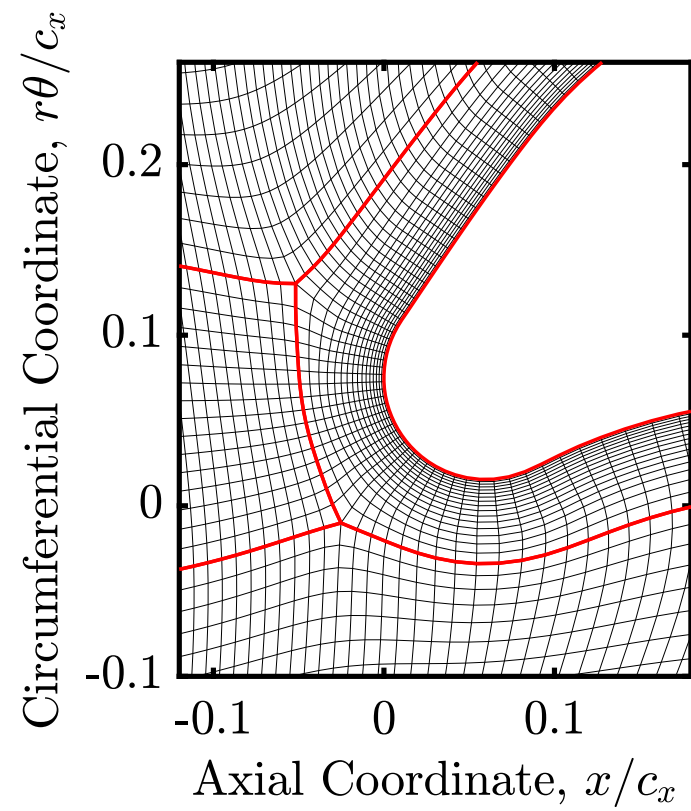

(c) Rotor leading edge

Fig. 4.3 Mid-span grid points of mesh used for non-film-resolved turbine computations. Block boundaries are highlighted in red. 


\subsection{Hybrid URANS-LES implementation}

The objective of the high-fidelity simulations is to predict the film effectiveness of cooling holes subject to given steady or unsteady main-stream boundary conditions. As discussed in Section 2.5, RANS simulations do not provide accurate results for film cooling, so an eddy-resolving approach is used which overcomes turbulence model limitations. The set-up and execution of these film-resolved simulations is now described.

A hybrid URANS-LES method is chosen in order to resolve the large turbulent structures necessary to predict film effectiveness, with length scales on the order of the hole diameter, while modelling small structures in the inner region of the boundary layer. A URANS layer, thickness $\delta_{l}$, is prescribed away from solid walls. The mixing-length turbulence model is used, with the turbulent viscosity, $\mu_{\text {turb }}$, proportional to a mixing length $l$ squared according to Prandtl's mixing-length hypothesis,

$$
\mu_{\mathrm{turb}}=(\kappa l)^{2} \sqrt{S_{i j} S_{i j}}, \quad S_{i j}=\frac{1}{2}\left(\frac{\partial V_{i}}{\partial x_{j}}+\frac{\partial V_{j}}{\partial x_{i}}\right),
$$

where $\kappa$ is the von Kármán constant, and $S_{i j}$ is the strain rate tensor. This is identical in form to the Smagorinsky model, with $\kappa l$ replacing a length scale based on the local grid spacing. The mixing length $l$ is prescribed algebraically, splitting the domain into URANS and LES zones according to,

$$
l=\left\{\begin{array}{llr}
y, & y \leq \delta_{l} / 2, & \text { near wall, } \\
-y+\delta_{l}, & \delta_{l} / 2<y \leq \delta_{l}, & \text { blend region, } \\
0, & y>\delta_{l}, & \text { free stream }
\end{array}\right.
$$

where $y$ is the distance to the nearest wall and $\delta_{l}$ is the URANS layer thickness as illustrated in Figure 4.4. This corresponds to a modelled fully-turbulent boundary layer for $y \leq \delta_{l} / 2$, a blending region for $\delta_{l} / 2<y \leq \delta_{l}$, and implicit LES with no modelled turbulence in $y>\delta_{l}$.

The approach is similar to that advocated by Tucker et al. (2012a,b). They argue that excessive dissipation in general-purpose compressible flow solvers, especially near walls, make fully-resolved LES with an explicit sub-grid scale model impractical on numerical grounds. Klostermeier (2008) demonstrated that the TBLOCK algorithms, on which TURBOstreAm is based, are suitable for implicit LES. Atkins and Kanjirakkad (2014) used a similar "practical" approach with the TURBOSTREAM solver to simulate buoyancy-driven flow in a rotating cavity without full wall resolution. 


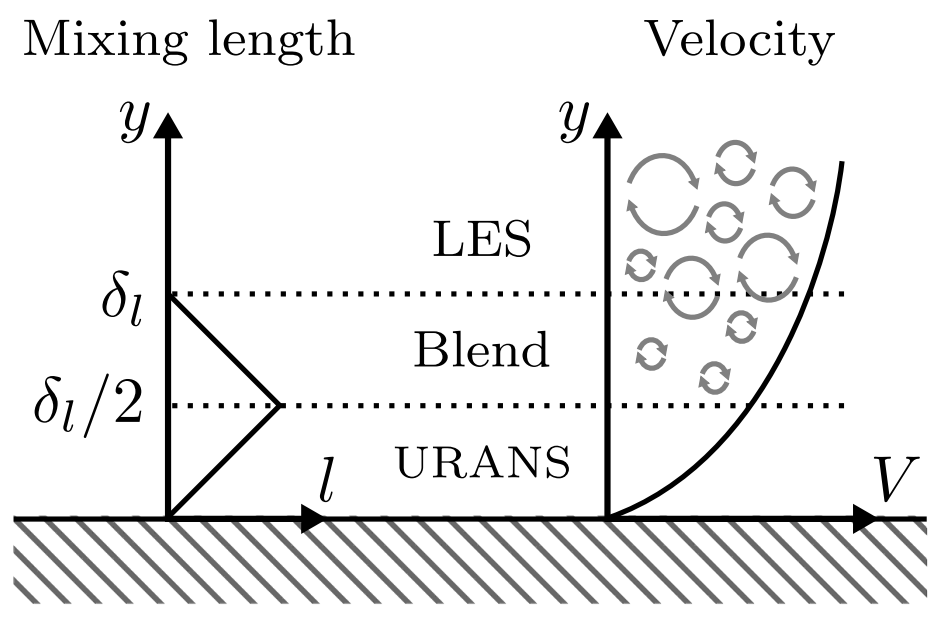

Fig. 4.4 Illustration of hybrid URANS-LES approach: variation of mixing length with wall distance and the associated computational zones.

The boundary between URANS and LES regions is a choice that must be made by the user. In this thesis, the URANS layer thickness is normalised against the cooling hole diameter and set to $\delta_{l} / D=8 \%$ based on calibration experience with flat plate cylindrical cooling hole test cases as described in Section 7.3. For representative boundary-layer thicknesses, this corresponds to a non-dimensional wall distance of $y^{+} \approx 125$ in the main stream, close to the corresponding value of $y^{+} \approx 100$ chosen by Tucker et al. (2012b).

In the implicit LES method, numerical dissipation drains kinetic energy at scales which cannot be resolved on the grid. This creates sensitivity to both mesh resolution, and the algorithms used in the flow solver.

The settings used for film-resolved computations in TURBOSTREAM 3.6.4 are listed in Table 4.1. In the author's experience, the usual stabilisation and convergence acceleration techniques suitable for URANS computations yield poor results in LES computations. So, negative feedback and multigrid are disabled by setting dampin $=1 \times 10^{9}$ and fmgrid $=0$ respectively.

A reduction in second-order smoothing to sfin $=0.1$ (from the default $\operatorname{sfin}=0.5$ ) was found to improve results by reducing dissipation at short length scales. To prevent 'ringing' or Gibbs phenomena at discontinuities in temperature, the adaptive smoothing is sensitised to temperature gradients (in addition to pressure gradients) by setting use_temperature_sensor $=1$. The number of dual time stepping iterations is fixed by setting dts_conv $=0$ and nstep_inner $=500$, because the built-in block-averaged convergence check was found to be unrepresentative of the required convergence level of small scales. The non-dimensional outer time step is set as $\Delta t V_{\infty} / D=0.02$ which corresponds to a Courant-Friedrichs-Lewy number $C F L \approx 0.5$, defined using the main- 
Table 4.1 TURBostream 3.6.4 variable configuration for hybrid URANS-LES filmresolved computations.

\begin{tabular}{ll}
\hline adaptive_smoothing & 1 \\
cfl & 0.4 \\
dampin & $1 \times 10^{9}$ \\
dts & 1 \\
dts_conv & 0 \\
facsafe & 0.25 \\
facsecin & 0.005 \\
fmgrid & 0 \\
ilos & 1 \\
nlos & 5 \\
nstep_inner & 500 \\
poisson_cfl & 0.7 \\
poisson_limit & 1 \\
poisson_nsmooth & 10 \\
poisson_nstep & 20000 \\
poisson_sfin & 0.01 \\
prandtl & 0.85 \\
precon & 0 \\
rfmix & 0.02 \\
rfvis & 0.2 \\
sfin & 0.1 \\
smooth_scale_directional_option & 1 \\
smooth_scale_dts_option & 1 \\
smooth_scale_precon_option & 0 \\
use_temperature_sensor & 1 \\
viscosity_law & 1 \\
wall_law & 0 \\
\hline frequency & $f$ \\
nstep_cycle & $50 f V_{\infty} / D$ \\
xllim & \\
\hline
\end{tabular}


stream velocity and streamwise grid spacing according to,

$$
C F L=\frac{V_{\infty} \Delta t}{\Delta x_{\infty}}
$$

A fixed turbulent Prandtl number prandt $1=0.85$ is prescribed based on boundary-layer experimental data (Kays, 1994). By setting viscosity_law $=1$, the dynamic viscosity, $\mu$, is varied with the 0.62 power of temperature according to,

$$
\mu=\mu_{\mathrm{ref}}\left(\frac{T}{T_{\mathrm{ref}}}\right)^{0.62}
$$

where subscript ref indicates a reference condition at $T_{\text {ref }}=288 \mathrm{~K}$.

\subsection{Film-resolved flat plate simulations}

Cases Hybrid URANS-LES predictions of film effectiveness are validated against two open-literature test cases as described in Chapter 7. These are cylindrical and laid-back fan-shaped cooling holes on a flat plate. The geometries are those tested by Saumweber et al. (2003) at the University of Karlsruhe, who reported film effectiveness data for a row of three holes in a transonic wind tunnel.

Domain and boundary conditions A schematic of the domain and boundary conditions for flat plate computations is shown in Figure 4.5. A Cartesian coordinate system origin is placed at the intersection of the hole axis and the cooled surface. Inlet boundary conditions for both coolant and main-stream flows are uniform stagnation temperatures, pressures and flow angles. The main-stream boundary conditions are applied at $x / D=-15$. The coolant boundary conditions are applied at $y / D=-20$, i.e. at the bottom of a plenum of height $17 D$. Main-stream turbulence is not included in these computations. A constant static pressure is specified on the exit plane at $x / D=20$. One cooling hole is simulated, and periodicity is imposed in the lateral $z$-direction, modelling an infinite row of holes. An inviscid wall is applied at a height $y / D=8$ from the plate. All walls are adiabatic.

Boundary layer trip Film effectiveness distributions are sensitive to the incoming boundary layer state (Bogard and Thole, 2006). To match the experiments, a correct upstream boundary condition, i.e. turbulent boundary layer, must be provided in the simulations. This is generated by using a trip to disturb an incoming uniform flow. A 


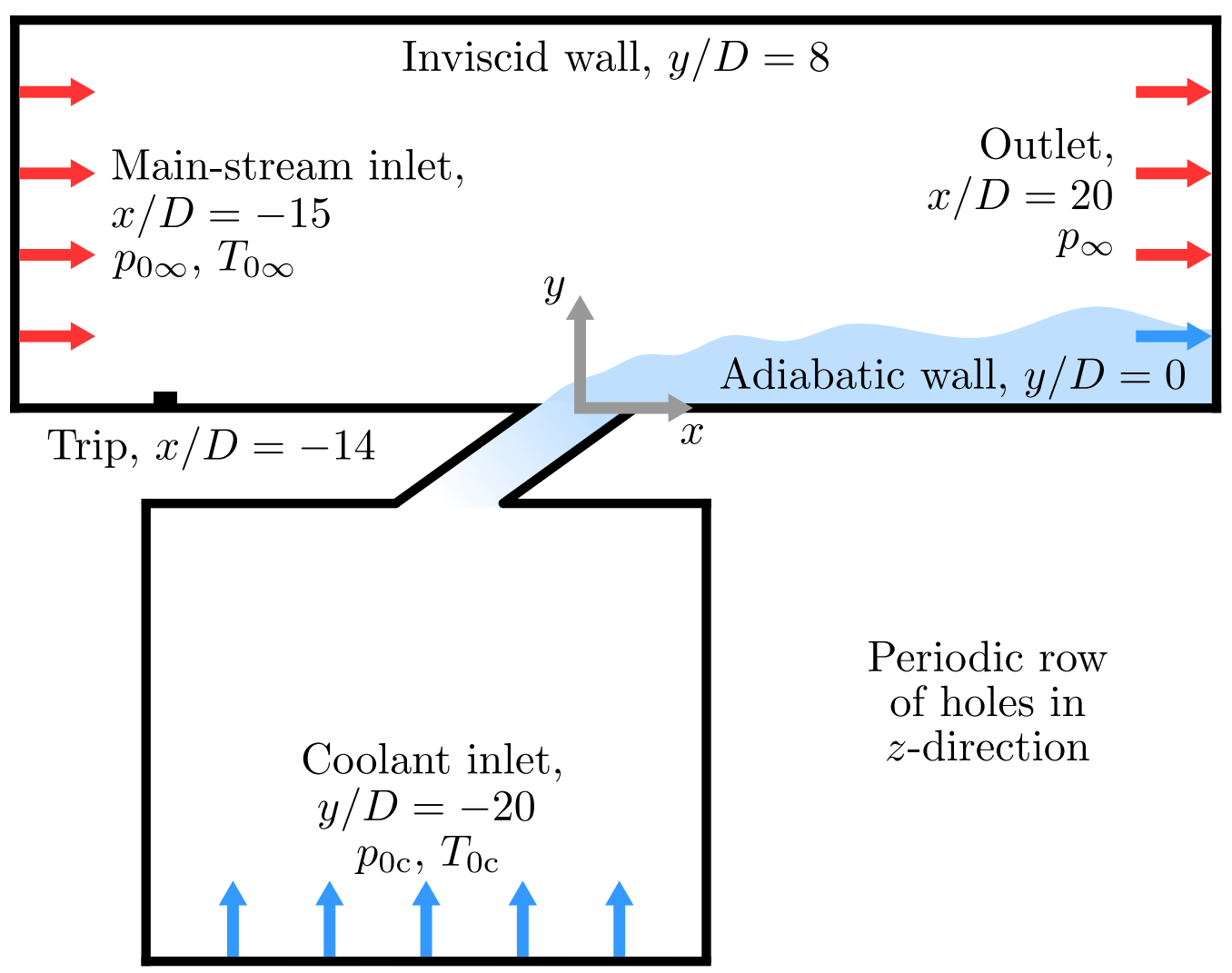

Fig. 4.5 Schematic of domain and boundary conditions for flat plate cooling hole simulations (not to scale). 
reversed streamwise velocity of $V_{x}=-0.7 V_{\infty}$ is imposed over a volume near the wall at $x / D=-14$. This creates an inflection point in the velocity profile, which is unstable and transitions the boundary layer to a fully-turbulent state. The volume extends across the entire hole pitch, with a length $0.1 D$ and a height $0.025 D$, one fifth of the target boundary layer displacement thickness. Results showing the efficacy of this method of generating inflow conditions are presented in Section 7.2.

Meshing Multi-block structured meshes are generated using the commercial software GRIDPRo (Program Development Company, 2017). A geometry and block topology are provided by the user as input, and an automatic algorithm optimises the grid for orthogonality and expansion ratio throughout the domain. O-meshes are used to wrap the inner and outer edges of cooling holes. The grid is clustered towards solid walls with an expansion ratio of 1.1. For the cylindrical hole case, the grid on the $y / D=0$ plane is shown in Figure 4.6, and on the $z / D=0$ plane in Figures 4.7, 4.8(a) and 4.8(b). Typical grid spacings for the near-hole region in wall units are $\Delta x^{+} \approx 50, \Delta y^{+} \approx 1$, and $\Delta z^{+} \approx 25$. Laminar wall functions are used, as the non-dimensional wall resolution is $\Delta y^{+}<5$ everywhere. Between 250 and 300 nodes are placed across the hole exit in all geometries. The mesh is refined for wall-normal locations $y / D<2$ to ensure adequate resolution of the coolant film, but coarsened outside of this in the main stream. These choices result in total mesh sizes of order 60 million nodes, with 15 million nodes in the cooling hole itself.

Mesh sensitivity In this implicit LES implementation, numerical dissipation acts like a sub-grid scale model which is inherently mesh-dependent. Therefore, mesh independence is not achieved until all structures are fully-resolved, i.e. in a direct numerical simulation, which would be prohibitive in computational expense. In LES, explicit filtering is required for refined meshes to converge to independence (Bose et al., 2010). For this reason, no mesh-independence study has been performed. However, the mesh resolution exceeds that typical in the literature, Section 2.5. For a back-to-back comparison with and without blade row interaction, as in Chapter 8 , this is deemed adequate.

Computation procedure First, a series of RANS simulations are performed to determine the hole pressure ratio required to give the desired coolant mass flow and hole operating point. The hybrid URANS-LES is then started from a converged RANS solution at the target pressure ratio and run with coarse time steps to flush transients for two main-stream flow-through times. Then, the simulation is run for two main-stream 


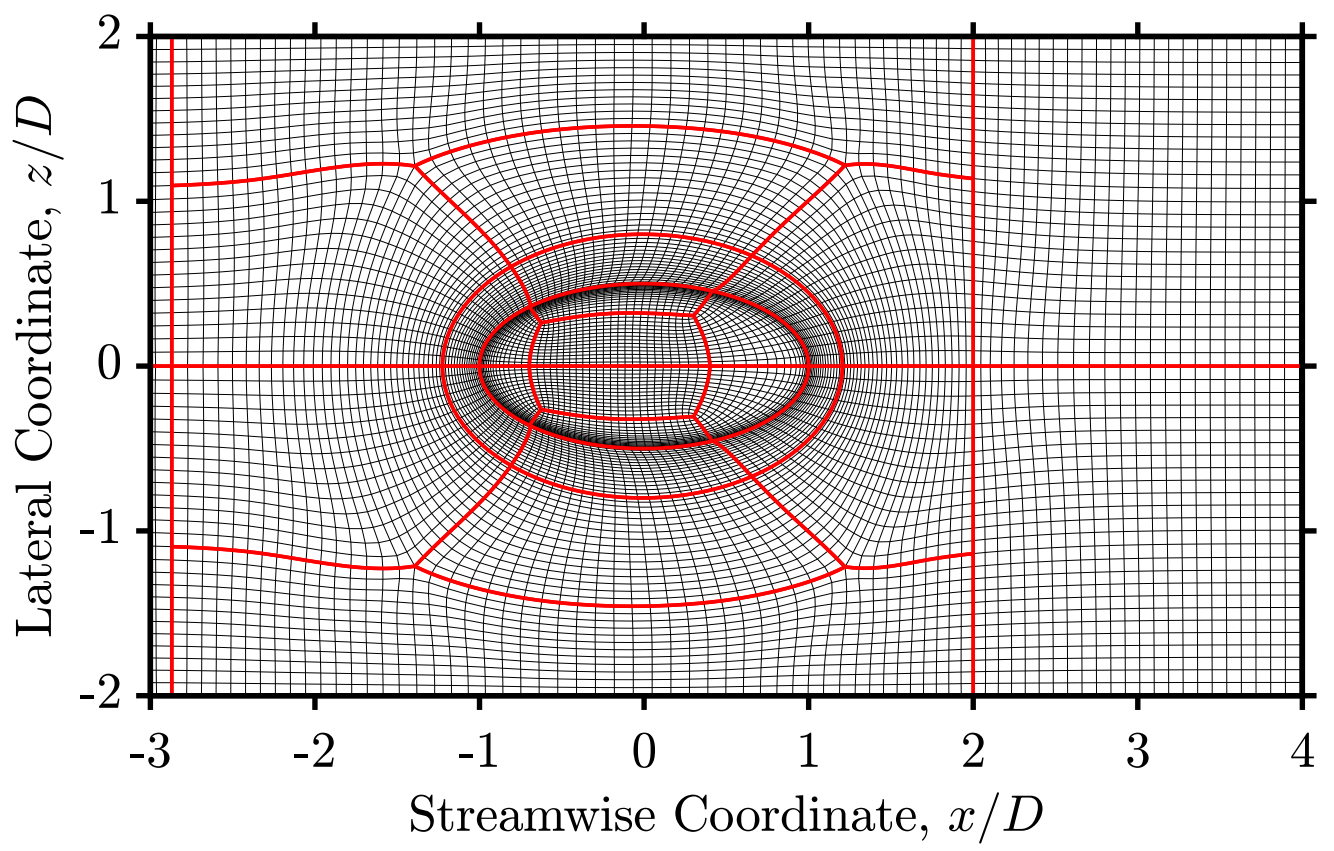

Fig. 4.6 Cylindrical cooling hole mesh topology and grid points on $y / D=0$ plane, every fourth grid line shown, block boundaries in red.

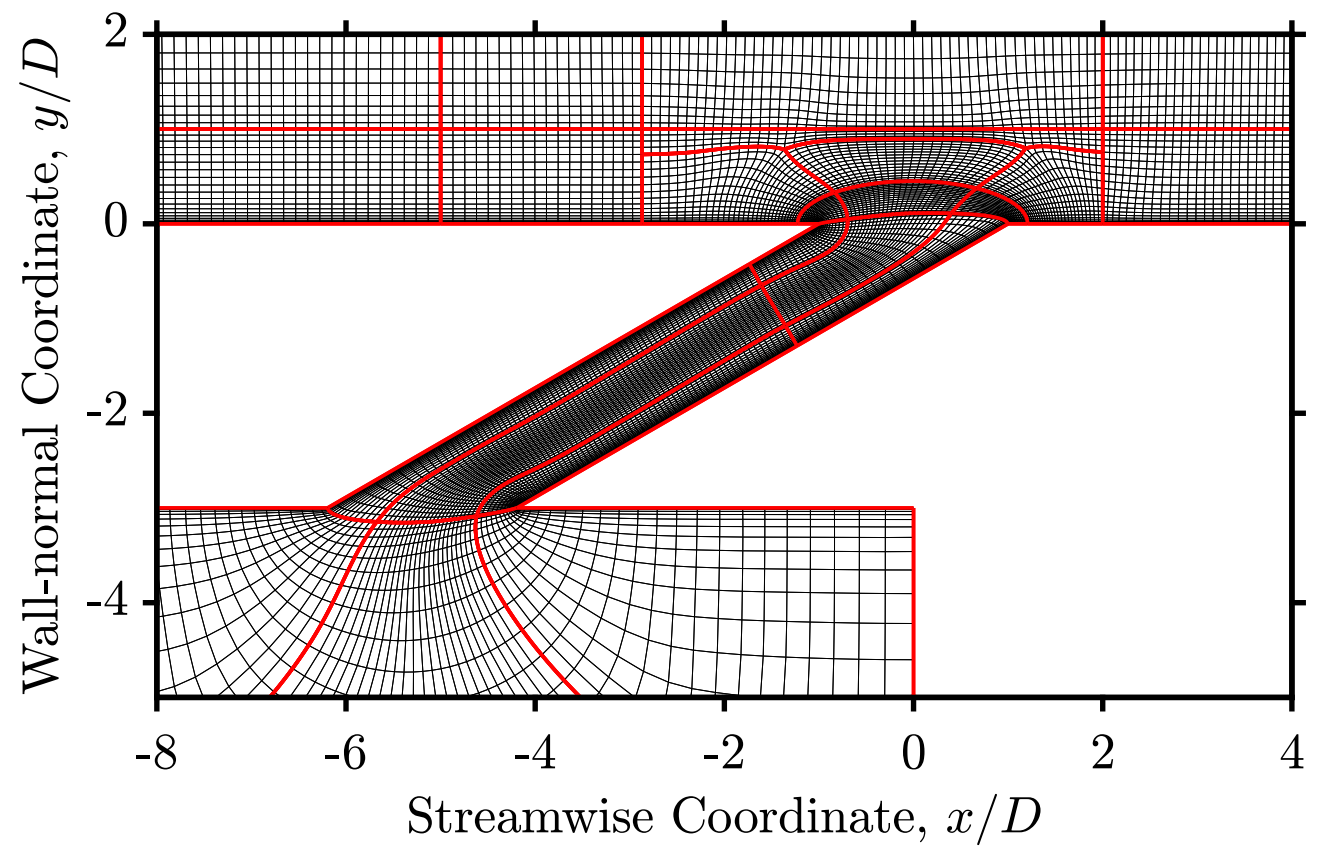

Fig. 4.7 Cylindrical cooling hole mesh topology and grid points on $z / D=0$ plane, every fourth grid line shown, block boundaries in red. 

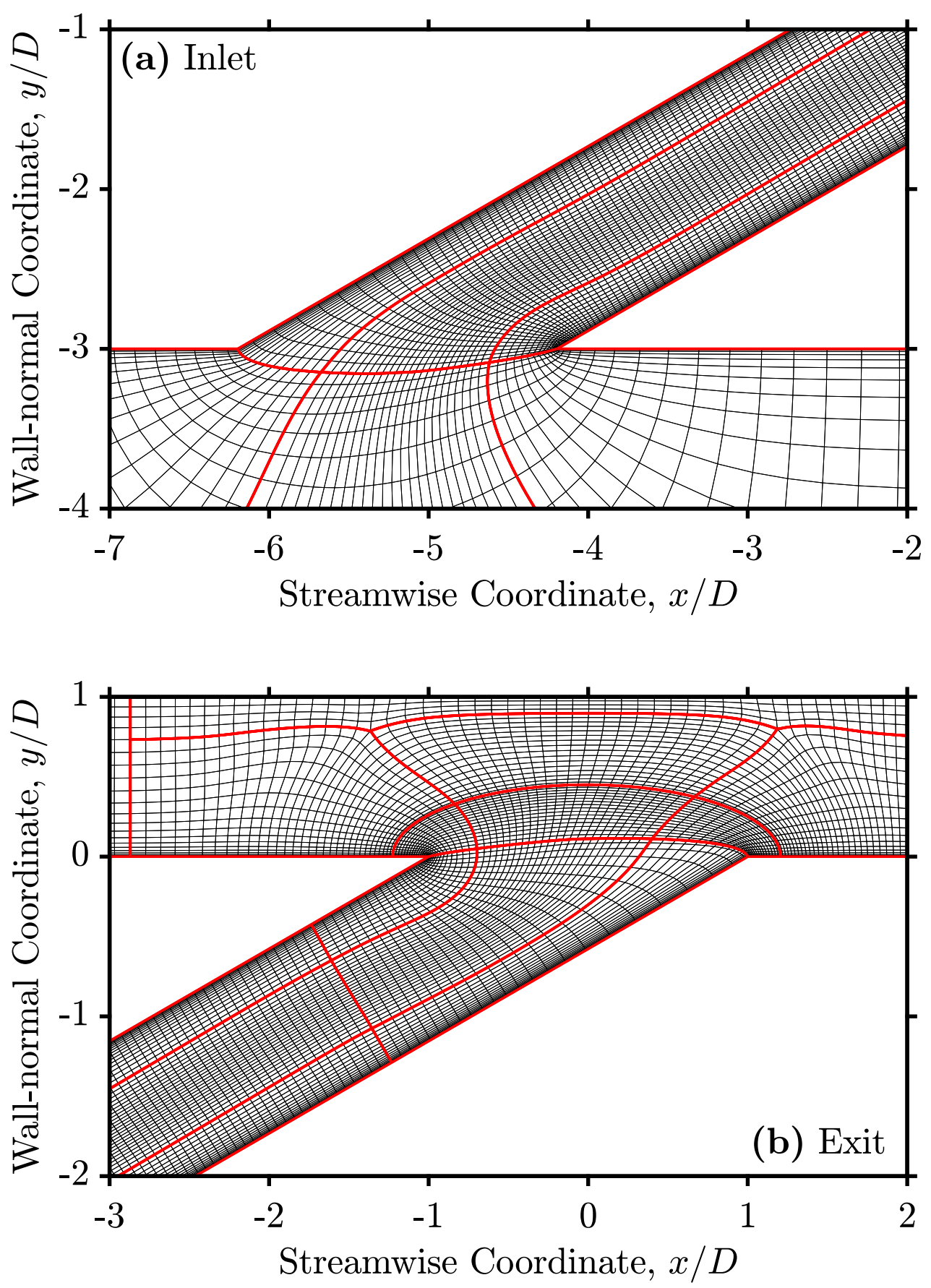

Fig. 4.8 Cylindrical cooling hole mesh topology and grid points detail on $z / D=0$ plane, (a) hole inlet, (b) hole exit. Every fourth grid line shown, block boundaries in red. 
flow-through times using the fine time step to reach a statistically-stationary state. Finally, averaging is performed over five restarts each lasting two flow-through times, a period of ten flow-through times in total. The standard deviation of the individual averages over each restart allows quantification of the uncertainty due to finite-time averaging, discussed in Section 7.3.

\subsection{Film-resolved cascade simulations}

Case Based on the flat plate computations, the method is applied to a single-stage 'linear cascade' of mid-span profiles from the same turbine used in the non-film-resolved simulations, with meshed rotor cooling holes. The rotor blade section is scaled up by $20 \%$ so that its pitch is equal to half a vane pitch, at constant pitch-to-chord ratio, keeping the leading edge at the same streamwise location. The spanwise extent of the domain is restricted to a single hole pitch, $P / D=4$, or $5 \%$ rotor axial chord.

Vane modelling The vane is treated with an approach similar to DES (Spalart, 2009), differing in that the RANS and LES zones are static and fixed before the computation starts. A mixing-length turbulence model is used throughout the boundary layers (not only in the inner region), with the mixing length prescribed using an algebraic limiter at $3 \%$ vane pitch as recommended by Denton (1992). The turbulence model is disabled at the vane trailing edge, where a finer grid allows resolution of an unsteady wake using implicit LES. As in the three-dimensional simulations outlined in Section 4.2, vane coolant is modelled using source patches, because resolving vane cooling holes is not necessary to produce a realistic wake.

Rotor modelling Rotor cooling holes are resolved using a hybrid URANS-LES approach, based on the flat plate computations. The thickness of the URANS layer is maintained at $\delta_{l} / D=8 \%$ as in the flat plate cases; the results are not thought to be sensitive to this choice. Based on the validation of the approach in Section 7.3, this modelling will yield reliable predictions of film effectiveness over the rotor blade.

Domain and boundary conditions A schematic of the domain and boundary conditions for the cascade case is shown in Figure 4.9. Scaling the rotor blade section allows the pitchwise $y$-extent to be reduced to a single vane pitch, compared to five vane pitches at the true vane/blade count ratio. In the $y$-direction, the stator is node-to-node matching periodic. In the rotor, differing cooling hole locations and mesh densities on each side 
$y$

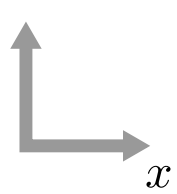

Mixing/sliding plane

Inviscid walls at spanwise boundaries $z / D= \pm P / 2 D$

Fig. 4.9 Schematic of domain and boundary conditions for film-resolved cascade simulations (not to scale).

of the blade require the use of a non-matching boundary condition in the pitchwise direction, where all nodes lie on the same periodic surface, but at arbitrary locations on that surface. A second-order accurate interpolation procedure is used to match the flow variables on each side of the periodic surface. Inviscid walls are used at the spanwise boundaries on $z / D= \pm P / 2 D$ in order to constrain the flow to a quasi-two-dimensional cascade. The main-stream inlet condition is uniform stagnation pressure and temperature extracted from the mid-span of an experimental radial traverse upstream of the vane. The downstream boundary condition is a constant static pressure, again taken from experimental data. Either a mixing or a sliding plane is used at the rotor-stator interface. Coolant stagnation pressure is chosen to give a pressure margin $4 \%$ over the mass-averaged stagnation pressure at the rotor-stator interface, in the rotor-relative frame. Similarly, coolant stagnation temperature is chosen to give a temperature ratio of 0.5 with respect to the mass-averaged stagnation temperature at the rotor-stator interface. The coolant is fed symmetrically from two inlet planes, situated 10 hole diameters above and below the spanwise location of the cooling holes. 
Meshing The flow solver used in this work requires a multi-block structured mesh. Hexahedral meshes also have the advantage of being less dissipative than comparable unstructured meshes using other element shapes, which is important for good LES resolution. However, the generation of a structured mesh for this cooled cascade geometry is non-trivial. The procedure used to construct a mesh comprises the following steps:

1. Extract section geometry: The mid-span vane and blade sections are taken from the full three-dimensional stage geometry. To remove discontinuities in surface curvature, which when resolved using a fine grid result in non-physical static pressure 'wiggles', the geometry is smoothed. Tenth-order polynomial fits are used to re-interpolate the profiles, reducing gradients in surface curvature while making negligible change to the overall geometry.

2. Add detail: The two-dimensional vane and blade profiles are imported into CAD software and extruded to form a three-dimensional solid. Cylindrical rotor cooling holes and feed plenums are added using the CAD modelling capabilities, with sizes and locations chosen to match those reported by Aoki (1999). The vane and blade are saved as triangulated stereolithography STL files.

3. Construct topology: The generation of the grid itself is performed using GRIDPRO software (Program Development Company, 2017). Geometry is imported, and a graphical user interface is used to construct a topology, which can be subsequently reused with similar geometries. Butterfly or O-mesh blocks are used to wrap the inner and outer edges of the cylindrical cooling holes. An O-mesh permits a local increase in resolution of the vane wake without propagation to the far field, shown in Figure 4.10. A 'nested' topology is used to refine the streamwise grid at the rotor surface by a factor of three, as proposed by Rajagopalan and Eiseman (2005) and illustrated in Figure 4.11.

4. Optimise mesh: The topology and geometry is taken as input, and the automatic GRIDPRO algorithm produces a mesh optimised for smoothness and orthogonality throughout the domain. The mesh for one side of the rotor domain (symmetric about $z / D=0$ ) is generated in multiple pieces; the smaller vane mesh can be generated in one piece. Supplementary GRIDPRO utilities are then used to join the pieces together, apply clustering at solid surfaces, merge blocks to reduce the total block count, and mirror the grid to form the full domain.

5. Finishing: Lastly, the grid is loaded into NUMECA IGG (Numeca International, 2014) for final processing. Blocks are merged to create one contiguous block spanning the 


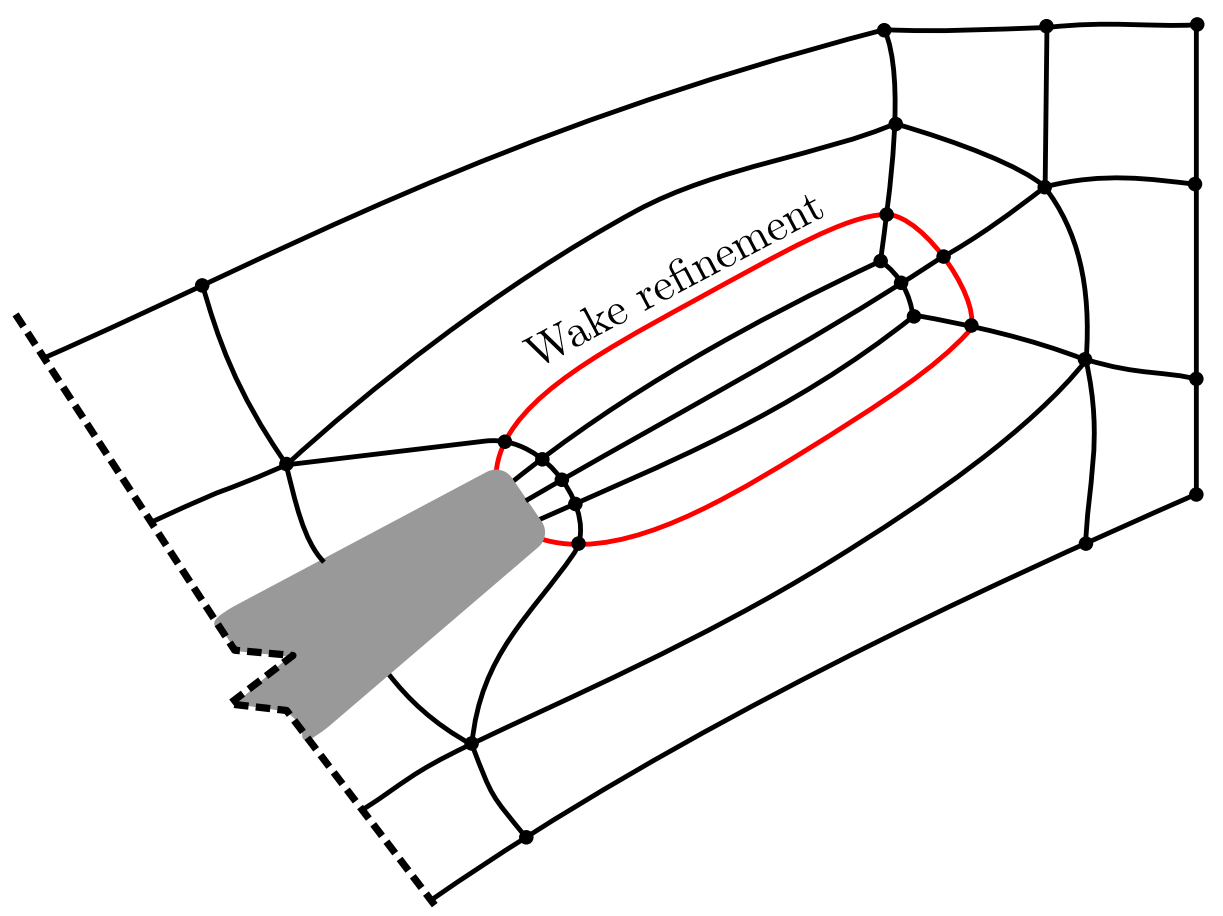

Fig. 4.10 Schematic of topology for wake refinement on a multi-block structured mesh. An increase in mesh density within the red O-mesh does not propagate to the far field.

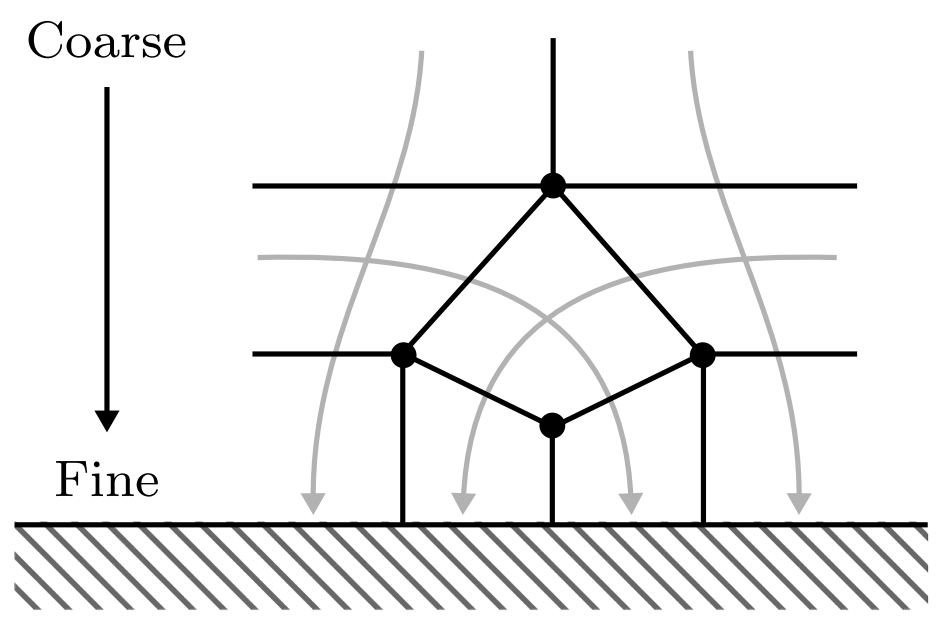

Fig. 4.11 Schematic of nested topology for wall refinement on a multi-block structured grid. When the pattern is repeated, one layer of nesting increases grid density by a factor of three. 
mixing plane between stator and rotor. Block orientations are modified to permit a non-matching boundary condition in the rotor, which are restricted to constant $k$-index surfaces by TURBostream. The grid is exported to PLOT3D and BCS files for CFD pre-processing.

Once the topology has been designed, one iteration of the meshing procedure takes several days, the most time-consuming part being the optimisation of the mesh pieces in GRIDPRO. The final, optimised mesh topology in the rotor is illustrated in Figure 4.12. The mesh comprises 65 blocks per vane passage and 272 per rotor passage, with 54 million and 331 million nodes respectively. When the rotor passage is duplicated once to form a periodic sector, the totals are 609 blocks and 717 million nodes. During computation, the mesh requires approximately 500GB of memory. In the fine time step simulation, one vane passing period requires three days of computing time on 64 NVIDIA Tesla P100 GPUs. A mesh coarsened by a factor of two in each direction is used for RANS and URANS simulations; this has 48 million nodes.

Computation procedure The use of implicit dual time stepping relaxes restrictions on the minimum stable time step. This allows a flexible approach to marching the solution forward in time to a statistically steady, periodic, converged state:

1. Begin with a converged RANS solution on a coarse mesh, propagate mixing-plane transients using URANS with 288 steps per vane passing period, for 40 periods.

2. Interpolate the instantaneous URANS solution onto the final mesh, refined by a factor of two in each direction. Resolve vane wake using approximately 72 steps per vortex shedding period, or 800 steps per vane passing, run for 16 vane passing periods.

3. Resolve rotor film cooling by setting $\Delta t V_{\infty} / D \leq 0.02$, as in the flat plate simulations. This criterion is most restrictive at the pressure- and suction-side holes, resulting in 7200 steps per vane passing. Run for 2 vane passing periods, to flush transients, and then as necessary to converge flow averages, approximately 8 vane passing periods. 

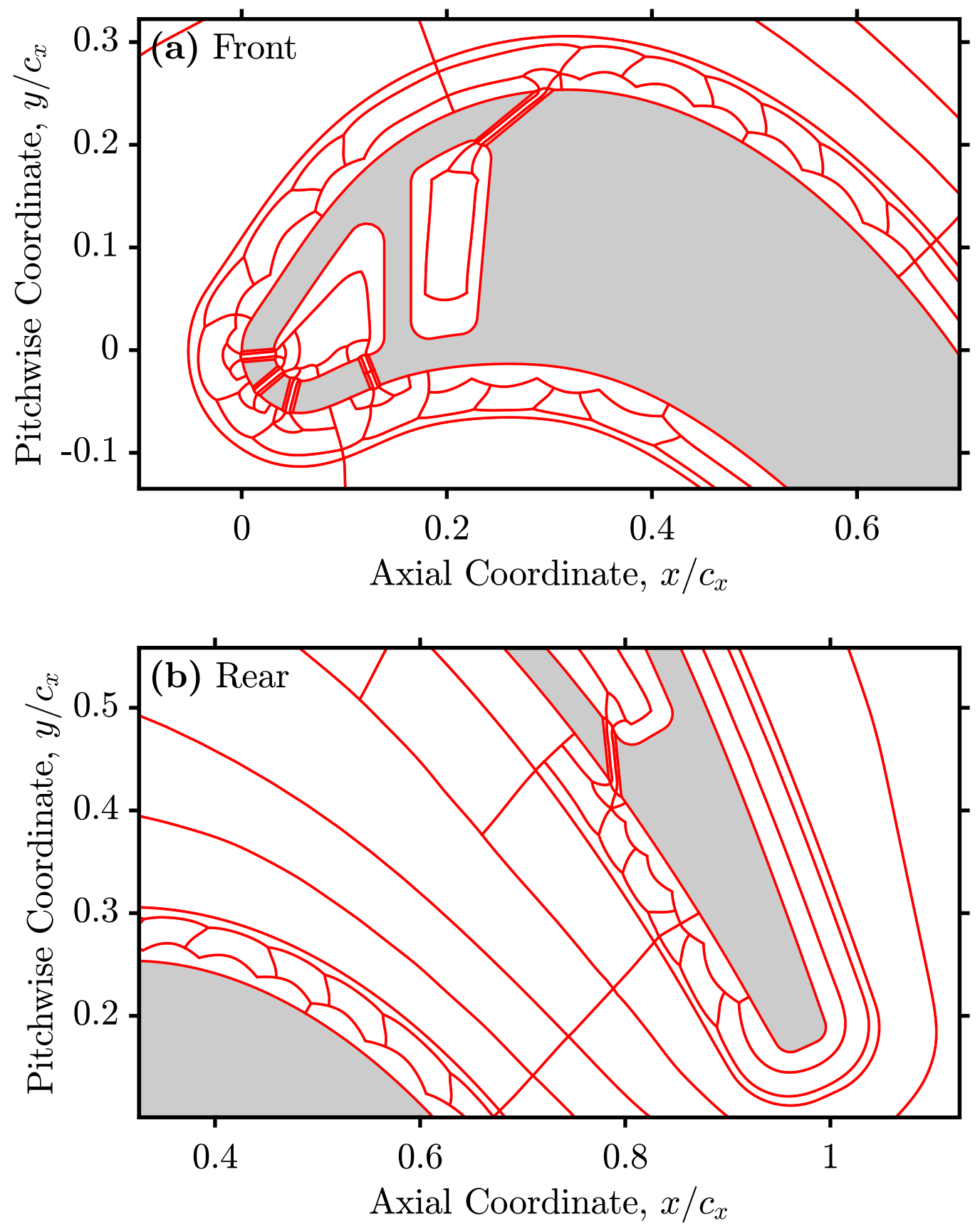

Fig. 4.12 Optimised block topology for rotor blade, at hole centre plane. 



\title{
Chapter 5
}

\section{Measurements of film cooling under unsteady boundary conditions}

\begin{abstract}
Experimental results showing the effect of main-stream unsteadiness on the performance of film cooling holes are presented. A simplified flat plate geometry with forcing from downstream allows control of boundary conditions, and hence the fluid-dynamic mechanisms governing unsteady blade row interaction effects on rotor film cooling can be identified.

Two film cooling hole geometries are tested, cylindrical and laid-back fan-shaped, at a series of time-averaged momentum flux ratios, with and without main-stream unsteadiness. Film effectiveness measurements quantify the effect of unsteadiness on cooling performance, and hot-wire measurements characterise the unsteady aerodynamic flow field.

Cylindrical holes are robust to unsteadiness at high momentum flux ratio, when the coolant is separated. At low momentum flux ratio, when coolant is attached to the wall, unsteadiness reduces film effectiveness by up to $31 \%$, because the attached coolant responds non-linearly to momentum flux ratio perturbations. Shaped holes are more robust to unsteadiness, which reduces film effectiveness by a maximum of $16 \%$, because they respond linearly at all momentum flux ratios.

It is concluded that, at a representative low reduced frequency, the effect of main-stream unsteadiness on film cooling is determined by non-linearity in the steady hole characteristic.
\end{abstract}




\subsection{Introduction}

In this Chapter, measurements of the heat transfer performance and aerodynamic flow fields of film cooling holes subject to main-stream unsteadiness are presented. The aim of the experiment is to determine the fluid-dynamic mechanisms governing the effect of unsteadiness. A simple low-speed, approximately isothermal, flat-plate geometry, allowing detailed measurements and precise control of boundary conditions, was developed to accomplish this aim with minimum complexity. This is the Unsteady Film Cooling Rig (UFC Rig). Details of the design and execution of the experiments are given in Chapter 3.

Two film cooling hole geometries are studied, cylindrical and laid-back fan-shaped, each at four time-averaged momentum flux ratios, $I R=0.2,0.6,1.0$ and 2.0. Typical turbine-representative values range from $0.5 \leq I R \leq 3.0$. The hole geometries, illustrated in Figure 5.1, are the same as those used by Saumweber et al. (2003). Non-dimensional geometry and boundary conditions for the tests are listed in Table 5.1. Similarity with real turbine conditions is discussed in detail in Section 3.1.2; in brief, all parameters excluding Mach number, temperature ratio, and turbulence intensity are matched to turbine conditions.

During commissioning, the inlet boundary layer properties were calculated from pneumatic probe traverses upstream of the cooling holes at $x / D=-10$. The mainstream turbulence intensity was measured using a hot-wire probe, and, as expected from the lack of upstream turbulence generating devices, confirmed to be negligible at $T u_{\infty} \leq 1 \%$.

The experimental results are divided into three sections. First, the main-stream unsteadiness generated in the rig is characterised using fast-response pressure transducer measurements, and an assessment is made of the resulting momentum flux ratio excursions of the cooling holes. Secondly, film effectiveness measurements are presented showing the effect of main-stream unsteadiness at different time-averaged momentum flux ratios, and physical explanations are given for the changes. Finally, phase-locked ensemble-averaged hot-wire measurements are used to examine the unsteady behaviour of the coolant film, confirming the arguments set out in the second section. 

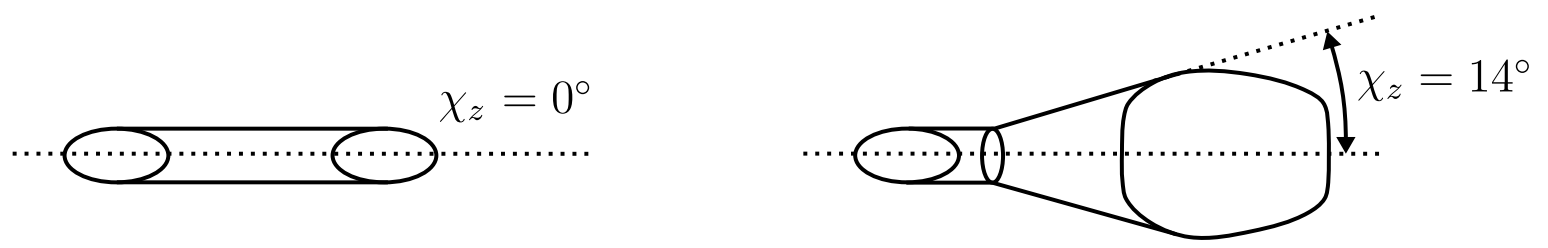

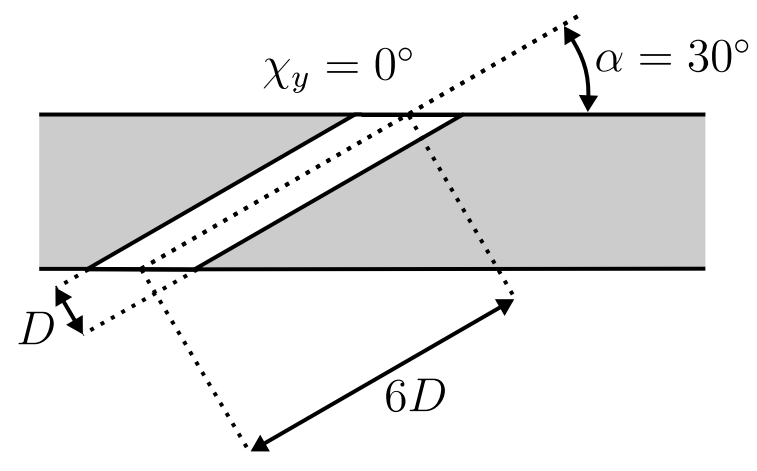

(a) Cylindrical

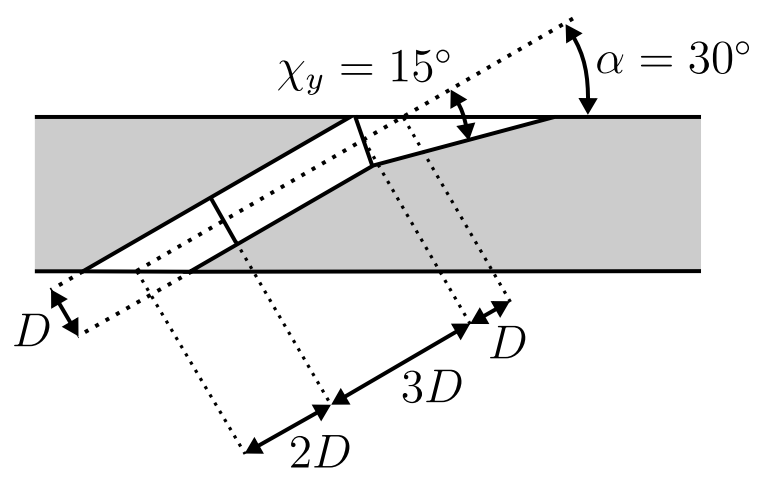

(b) Laid-back fan-shaped

Fig. 5.1 Hole geometries for experimental tests, after Saumweber et al. (2003).

Table 5.1 Non-dimensional geometry and boundary condition parameters for tests in the Unsteady Film Cooling Rig.

\begin{tabular}{ll} 
Hole lateral expansion angle, $\chi_{z}$ & $0^{\circ}, 14^{\circ}$ \\
Hole wall-normal expansion angle, $\chi_{y}$ & $0^{\circ}, 15^{\circ}$ \\
Hole inclination angle, $\alpha$ & $30^{\circ}$ \\
Hole pitch-to-diameter ratio, $P / D$ & 4 \\
Hole length-to-diameter ratio, $L / D$ & 6 \\
Main-stream Mach number, $M a_{\infty}$ & 0.03 \\
Main-stream Reynolds number, $R e_{\infty}$ & 15000 \\
Main-stream turbulence intensity, $T u_{\infty}$ & $1 \%$ \\
Boundary-layer displacement thickness, $\delta_{*} / D$ & 0.074 \\
Boundary-layer shape factor, $\delta_{*} / \theta$ & 1.41 \\
Coolant temperature ratio, $T_{0 \mathrm{c}} / T_{0 \infty}$ & 1.1 \\
Coolant momentum flux ratio, $I R$ & $0.2,0.6,1.0,2.0$ \\
Reduced frequency, $\kappa_{\infty}$ & 0.005 \\
Pressure fluctuation amplitude, $\Psi$ & $0.0,0.4$ \\
\hline
\end{tabular}




\subsection{Unsteadiness amplitude}

In this section, the time-varying boundary conditions imposed on film cooling holes in the UFC Rig are characterised. The rig uses a rotating blockage to create an unsteady static pressure in the working section, modelling potential field interactions occurring in a real turbine rotor. A target normalised pressure fluctuation amplitude of $\Psi=0.4$, defined in Equation (3.4), was chosen as representative based on URANS turbine stage computations (described in Chapter 6).

In order to verify the actual pressure fluctuation amplitude created in the Rig, a pair of fast-response pressure transducers were used to measure the instantaneous plenum and main-stream static pressures. Figure 5.2(a) shows simultaneous traces for the mainstream (black) and plenum (colours) at all tested time-averaged momentum flux ratios. The static pressure is plotted as a pressure coefficient defined,

$$
C_{p}(t)=\frac{p_{0 \infty}-p(t)}{p_{0 \infty}-p_{\infty}}
$$

The main-stream pressure fluctuates by \pm 0.6 main-stream dynamic heads, and simultaneously the plenum pressure also fluctuates by \pm 0.25 main-stream dynamic heads due to the unsteady response of the coolant supply system.

To determine the instantaneous pressure drop across the cooling hole, which governs mass flow fluctuations, the two signals can be subtracted according to,

$$
\Delta C_{p}(t)=\frac{p_{\mathrm{c}}(t)-p_{\infty}(t)}{p_{0 \infty}-p_{\infty}}
$$

as plotted in Figure 5.2(b). The mean-to-peak variation in $\Delta C_{p}$ is identically equal to the normalised pressure fluctuation amplitude, $\Psi$. The unsteady hole pressure drop is smooth and quasi-sinusoidal at the pressure fluctuator rotation frequency. Values of $\Psi$ are tabulated for all tests in Table 5.2. The pressure fluctuation amplitude is a weak function of flow conditions, varying by $\pm 10 \%$, taking a value of $\Psi=0.36$ at $I R=0.2$ with the shaped hole geometry, and rising to $\Psi=0.44$ at $I R=2.0$ with the cylindrical hole geometry. The artificial main-stream unsteadiness created in the Rig is representative of turbine conditions with $\Psi \approx 0.4$.

For incompressible flow, the momentum flux ratio may be written,

$$
I R=\frac{\rho_{\mathrm{c}} V_{\mathrm{c}}^{2}}{\rho_{\infty} V_{\infty}^{2}}=\Delta C_{p} C_{\mathrm{d}}^{2}
$$



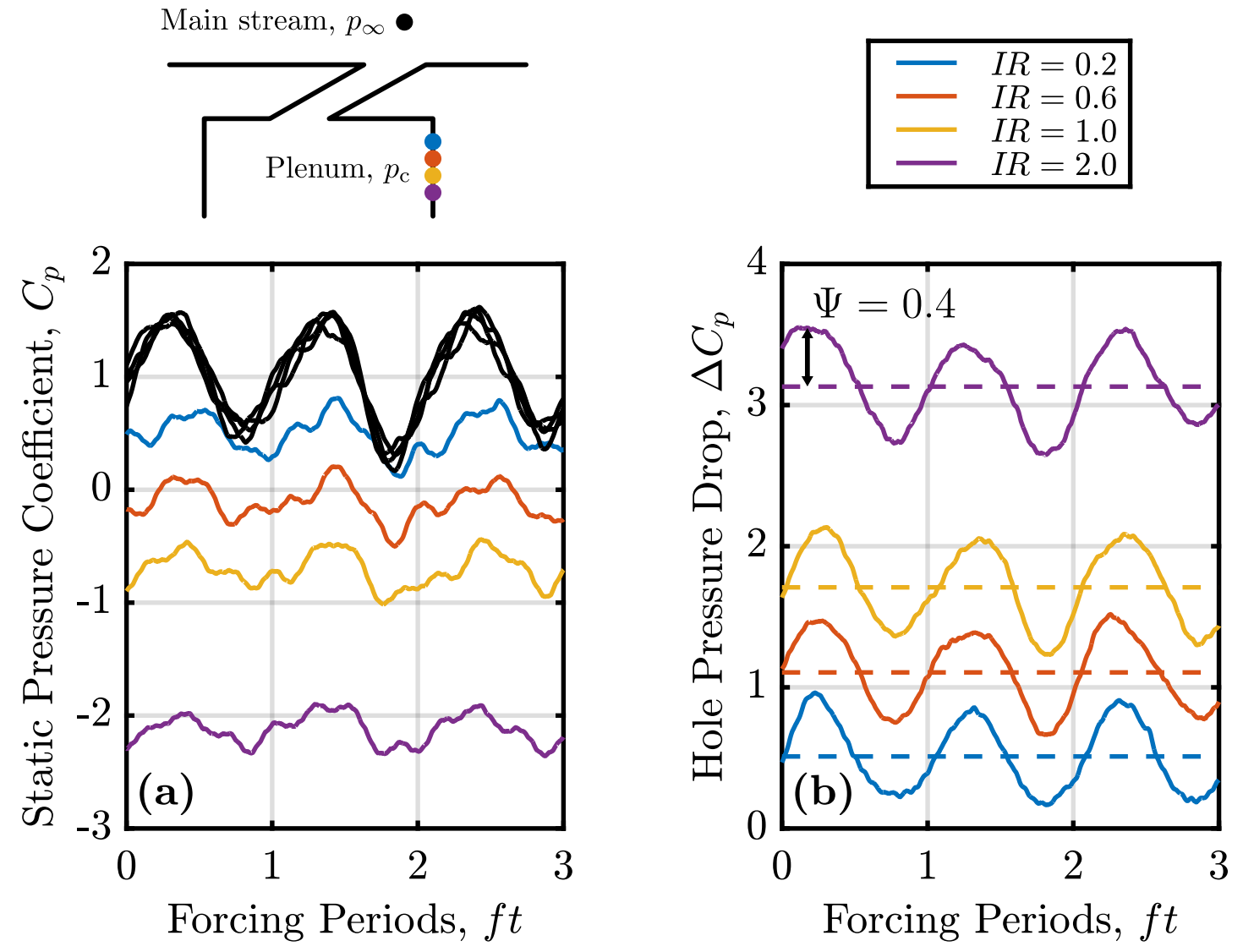

Fig. 5.2 Measurements of static pressure unsteadiness: (a) Instantaneous static pressures in main stream and plenum; (b) Instantaneous pressure drop across the hole.

Table 5.2 Estimated momentum flux ratio excursions for all test conditions from discharge coefficient and main-stream pressure fluctuation amplitude data.

\begin{tabular}{l|cccc|cccc} 
& \multicolumn{4}{|c|}{ Cylindrical } & \multicolumn{4}{c}{ Shaped } \\
\hline Momentum Flux Ratio, IR & 0.2 & 0.6 & 1.0 & 2.0 & 0.2 & 0.6 & 1.0 & 2.0 \\
Pressure Amplitude, $\Psi$ & 0.41 & 0.41 & 0.43 & 0.44 & 0.34 & 0.36 & 0.38 & 0.40 \\
Discharge Coefficient, $C_{\mathrm{d}}$ & 0.67 & 0.74 & 0.77 & 0.79 & 0.76 & 0.85 & 0.87 & 0.88 \\
Unsteady IR Excursion, $\widetilde{I R}$ & 0.18 & 0.21 & 0.26 & 0.27 & 0.20 & 0.26 & 0.28 & 0.31 \\
\hline
\end{tabular}


where $C_{\mathrm{d}}$ is the hole discharge coefficient. The discharge coefficient characterises losses in the hole as the ratio of actual mass flow to ideal mass flow assuming a one-dimensional isentropic expansion between the coolant plenum and main-stream static pressures, or for incompressible flow,

$$
C_{\mathrm{d}}=\frac{\text { actual velocity }}{\text { ideal velocity }}=\frac{V_{\mathrm{c}}}{\sqrt{2\left(p_{\mathrm{c}}-p_{\infty}\right) / \rho_{\mathrm{c}}}} .
$$

Taking the discharge coefficient as approximately constant during unsteady excursions, the amplitude of momentum flux ratio excursions, $\widetilde{I R}$, is given by substituting $\Psi$ for $\Delta C_{p}$ in Equation (5.3),

$$
\widetilde{I R} \approx \Psi C_{\mathrm{d}}^{2}
$$

Discharge coefficients calculated from test data are also shown in Table 5.2. From the measured pressure fluctuation amplitude and discharge coefficient data, application of Equation (5.5) allows estimation of the excursions in momentum flux ratio in each test, also shown in Table 5.2. Across all tests, the momentum flux ratio excursions are $0.18 \leq \widetilde{I R} \leq 0.31$

\subsection{Film effectiveness}

In this section, film effectiveness data are presented to quantify the effect of main-stream unsteadiness on film cooling performance at different time-averaged hole operating points. The two tested geometries, cylindrical and shaped, are discussed in turn.

\subsubsection{Cylindrical holes}

Contours of film effectiveness for cylindrical cooling holes with a steady main-stream are shown in Figure 5.3. The contours exhibit the expected shape and trend with momentum flux ratio as established in the literature. Effectiveness is highest on the centreline and decays in the lateral direction. The maximum centreline effectiveness is $\varepsilon_{\mathrm{f}}=0.66$ at $x / D \approx 6$ for $I R=0.2$, in Figure 5.3(a). Coolant coverage, where effectiveness remains above an arbitrary threshold value $\varepsilon_{\mathrm{f}} \geq 0.1$, extends to $z / D= \pm 0.96$ at $I R=0.2$. As the momentum flux ratio is increased, both the centreline effectiveness and coolant coverage reduce, because the coolant momentum increases, causing separation from the wall. At the highest tested momentum flux ratio, $I R=2.0$, in Figure 5.3(d), the peak centreline effectiveness has dropped to $\varepsilon_{\mathrm{f}}=0.24$, and lateral coolant coverage has halved to $z / D= \pm 0.45$ 
Film Effectiveness, $\varepsilon_{\mathrm{f}}$
0
0.2
0.4
0.6
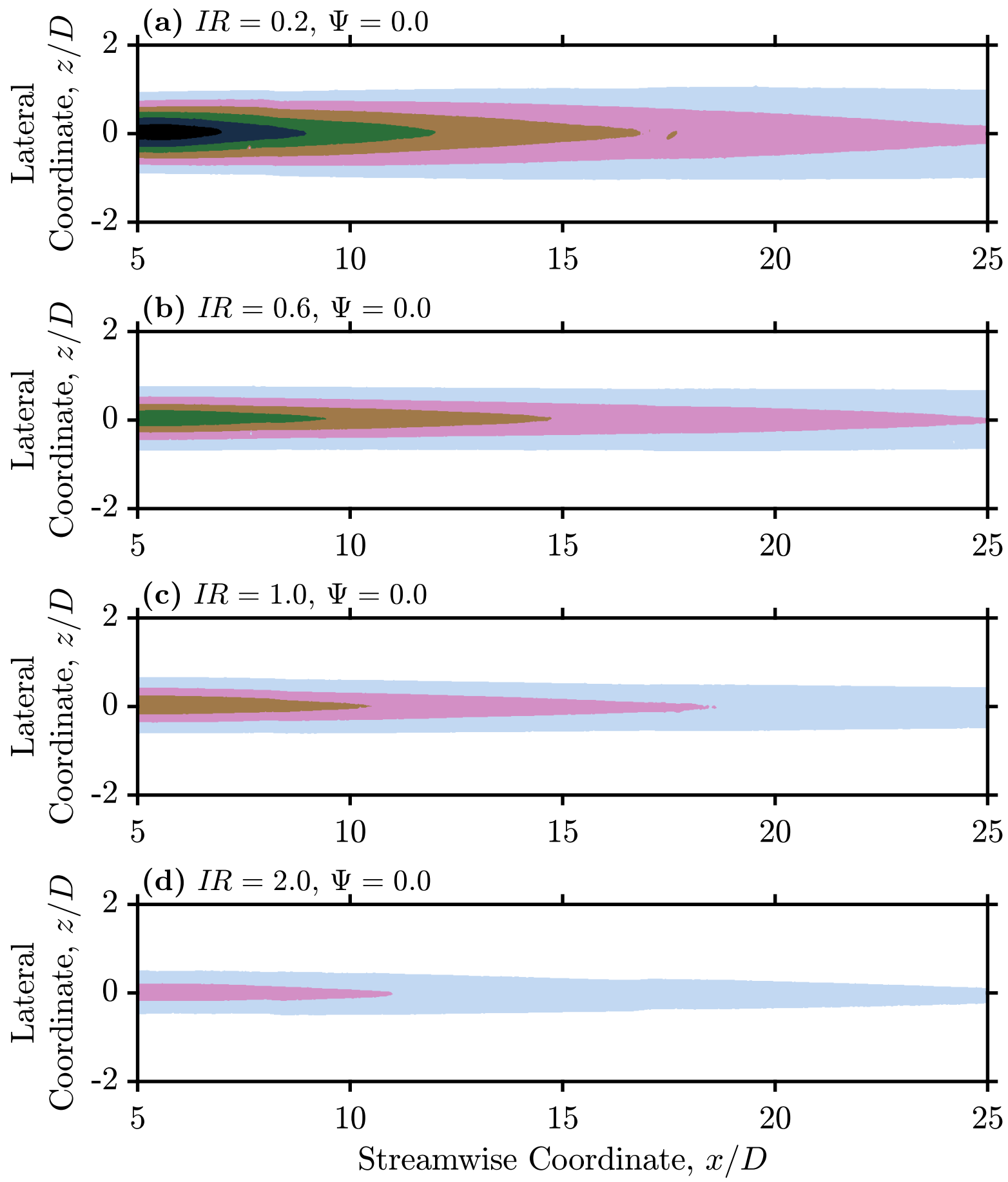

Fig. 5.3 Contours of measured film effectiveness for cylindrical holes under steady main-stream boundary conditions, $\Psi=0.0$, at momentum flux ratios (a) $I R=0.2$, (b) $I R=0.6$, (c) $I R=1.0$, (d) $I R=2.0$. 
Figure 5.4 shows the corresponding contours of film effectiveness with the pressure fluctuator active and producing unsteady main-stream boundary conditions with $\Psi=0.4$. The time-averaged boundary conditions are held constant. Comparing Figures 5.3(a) and 5.4(a), at $I R=0.2$ the local film effectiveness is reduced by up to 0.18 at all surface locations by main-stream unsteadiness, and lateral coolant coverage is reduced by $17 \%$ to $z / D= \pm 0.8$. The film effectiveness distribution is more similar to that at a higher momentum flux ratio with steady boundary conditions, Figure 5.3(b). However, with an unsteady main-stream at $I R \geq 0.6$, there are no qualitative differences between the contours.

The two-dimensional surface data may be reduced into one-dimensional streamwise distributions by area-averaging in the lateral direction to produce the laterally-averaged film effectiveness, $\overline{\varepsilon_{\mathrm{f}}}$. Figure 5.5 displays the reduced data of Figures 5.3 and 5.4 together.

Figure 5.5 shows that, at high momentum flux ratio, $I R \geq 1.0$, the film effectiveness distributions with and without unsteadiness are identical to within the measurement uncertainty of $C I\left(\overline{\varepsilon_{\mathrm{f}}}\right) \leq 0.008$. That is, imposing unsteady boundary conditions produces no effect distinguishable with the present apparatus. At low momentum flux ratio, $I R=0.2$, main-stream unsteadiness reduces laterally-averaged film effectiveness by between 0.03 and 0.06 , or $26 \%$ to $31 \%$. At $I R=0.6$, the results show an improvement in performance with main-stream unsteadiness of approximately 0.01 or $10 \%$ in film effectiveness, about twice the measurement uncertainty of $C I\left(\overline{\varepsilon_{\mathrm{f}}}\right) \leq 0.005$ in this case.

The impact of unsteadiness can be explained with reference to the steady performance characteristic in Figure 5.6. This is the local laterally-averaged film effectiveness at a fixed streamwise location, $x / D=8$, plotted as a function of momentum flux ratio. The black line shows measured data for a range of operating points under steady conditions. At low reduced frequency, the hole will behave quasi-steadily and the instantaneous performance will follow the black line during unsteady fluctuations. The filled circles identify steady operating points from Figure 5.5, and the open circles time-averaged unsteady operating points.

The hole characteristic in Figure 5.6 is approximately linear for $I R \geq 0.8$, when coolant is fully separated from the wall (Bogard and Thole, 2006). This means that positive and negative perturbations in momentum flux ratio produce opposing perturbations in film effectiveness, which cancel each other out when time-averaged. The result is that unsteady movement about the $I R=1.0$ and $I R=2.0$ operating points does not affect time-averaged film effectiveness.

The hole characteristic in Figure 5.6 is non-linear around the performance peak at $I R=0.2$. Both positive and negative perturbations in momentum flux ratio decrease film 
Film Effectiveness, $\varepsilon_{\mathrm{f}}$
0
0.2
0.4
0.6
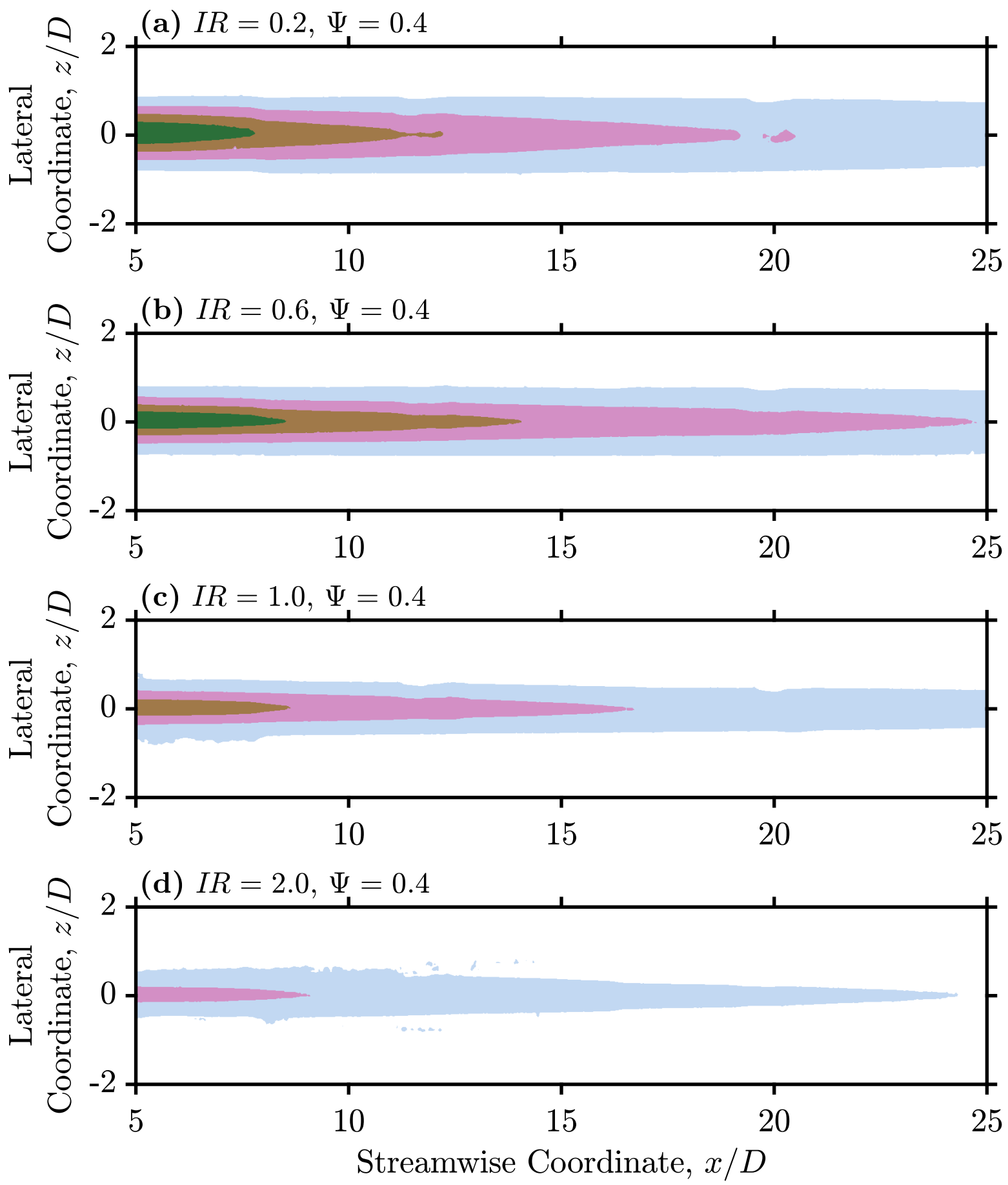

Fig. 5.4 Contours of measured film effectiveness for cylindrical holes under unsteady main-stream boundary conditions, $\Psi=0.4$, at momentum flux ratios (a) $I R=0.2$, (b) $I R=0.6$, (c) $I R=1.0$, (d) $I R=2.0$. 


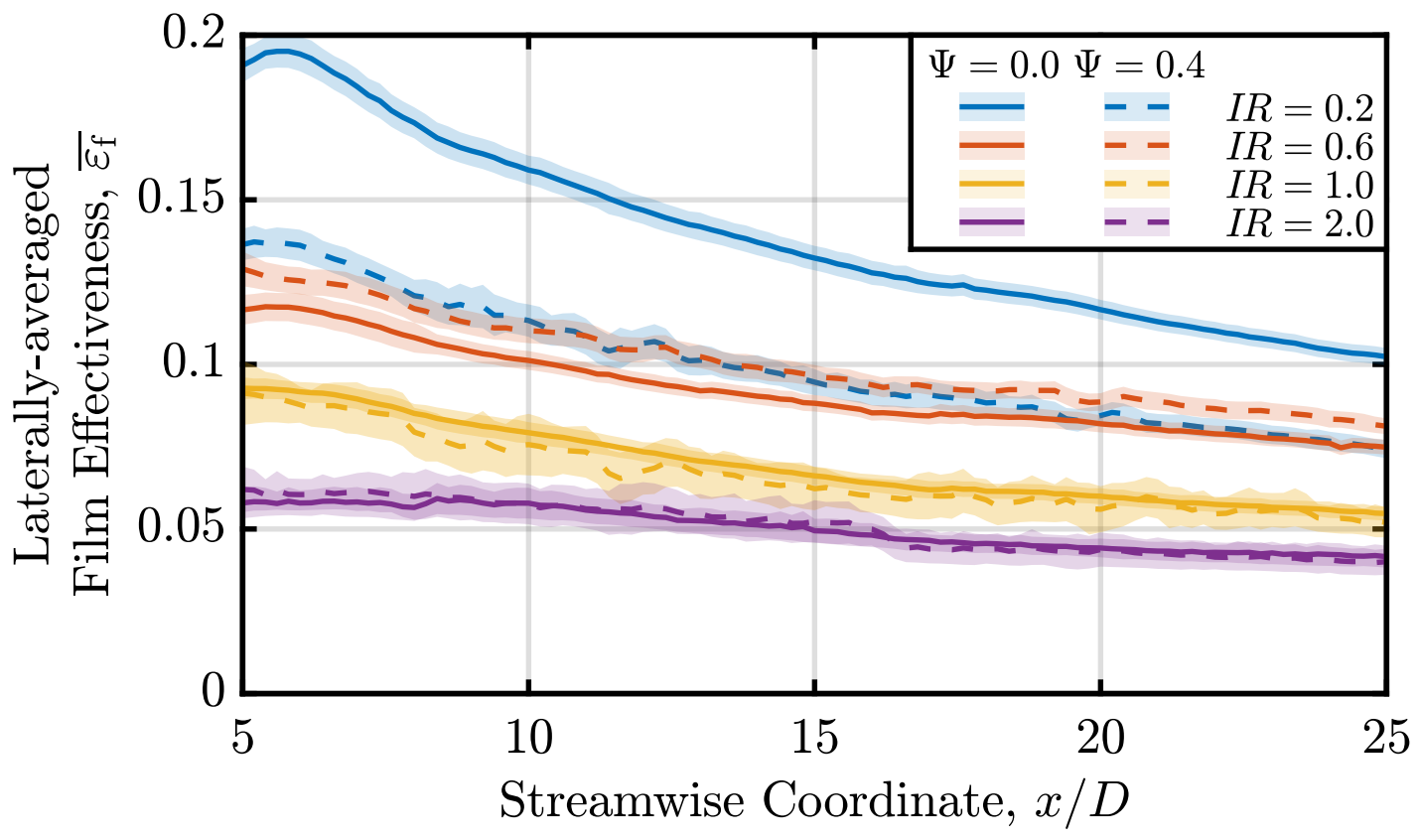

Fig. 5.5 Streamwise distributions of measured laterally-averaged film effectiveness distributions for cylindrical holes, with main-stream unsteadiness, $\Psi=0.4$, and without main-stream unsteadiness, $\Psi=0.0$.

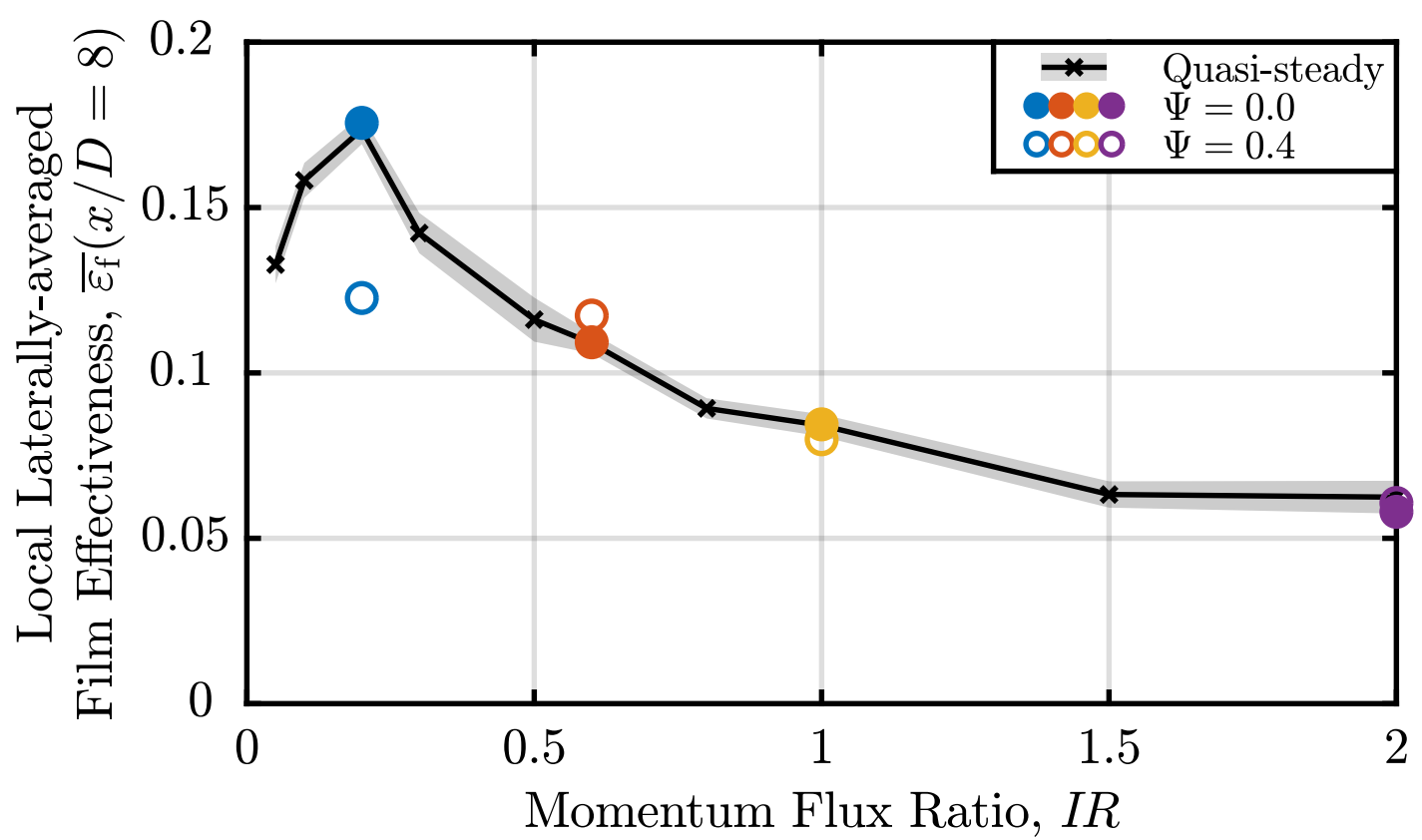

Fig. 5.6 Quasi-steady momentum flux ratio characteristic for laterally-averaged film effectiveness at $x / D=8$, cylindrical holes, with steady and time-averaged unsteady operating points marked. 
effectiveness. If the momentum flux ratio is reduced, effectiveness reduces because coolant mass flow reduces. If the momentum flux ratio is increased, the coolant separates from the wall, also reducing effectiveness. Time-averaging, the perturbations do not cancel out. The effect of unsteadiness is a reduction of $30 \%$ in film effectiveness at constant time-averaged boundary condition.

At $I R=0.6$, the coolant has started to lift off but is not yet fully-separated from the wall. Although the characteristic is approximately linear, Figure 5.6, there is some positive curvature - unsteady movement on this part of the characteristic would tend to increase film effectiveness, consistent with the marginal increase observed in the experiments. Similar behaviour has also been reported in the literature: Seo et al. (1999) found that main-stream unsteadiness increased film effectiveness of a separated cylindrical hole at $I R=1.1$, because unsteadiness caused the coolant to be instantaneously attached during the forcing cycle, when it would be constantly detached in steady state.

\subsubsection{Shaped holes}

Contours of film effectiveness for the laid-back fan-shaped film cooling holes under steady boundary conditions are shown in Figure 5.7. The expansion of the hole exit produces increased lateral spreading, so that lateral gradients in film effectiveness are reduced compared to the cylindrical hole data in Figure 5.3, and coolant coverage extends across the entire hole pitch. Diffusion through the expanding hole area reduces coolant momentum, such that coolant does not separate from the wall, and film effectiveness levels increase monotonically with momentum flux ratio.

Contours of film effectiveness for the laid-back fan-shaped film cooling holes with unsteady boundary conditions at $\Psi=0.4$ are shown in Figure 5.8. Aside from a somewhat reduced level of film effectiveness, by up to 0.05 , for $x / D<15$ at $I R=0.2$ in Figure 5.8(a), the contours are qualitatively similar with and without main-stream unsteadiness.

Streamwise distributions of laterally-averaged film effectiveness for the shaped holes are shown in Figure 5.9, with both steady and unsteady main-stream boundary conditions. Due to increased flow unsteadiness and a longer extrapolation range (discussed in Section 3.3.4), the data at $I R=2.0$ have the largest measurement uncertainty of $C I\left(\overline{\varepsilon_{\mathrm{f}}}\right) \approx 0.04$. Discounting the $I R=2.0$ data for $x / D \leq 8$, unsteadiness produces a consistent reduction in laterally-averaged film effectiveness of between 0.01 and 0.03 . This drop is indistinguishable from the measurement uncertainty for $I R \geq 0.6$, but exceeds the measurement uncertainty at $I R=0.2$.

The shaped hole performance characteristic, laterally-averaged film effectiveness at $x / D=8$ as a function of momentum flux ratio, is shown in black on Figure 5.10. Steady 
Film Effectiveness, $\varepsilon_{\mathrm{f}}$

$\begin{array}{llll}0 & 0.2 & 0.4 & 0.6\end{array}$
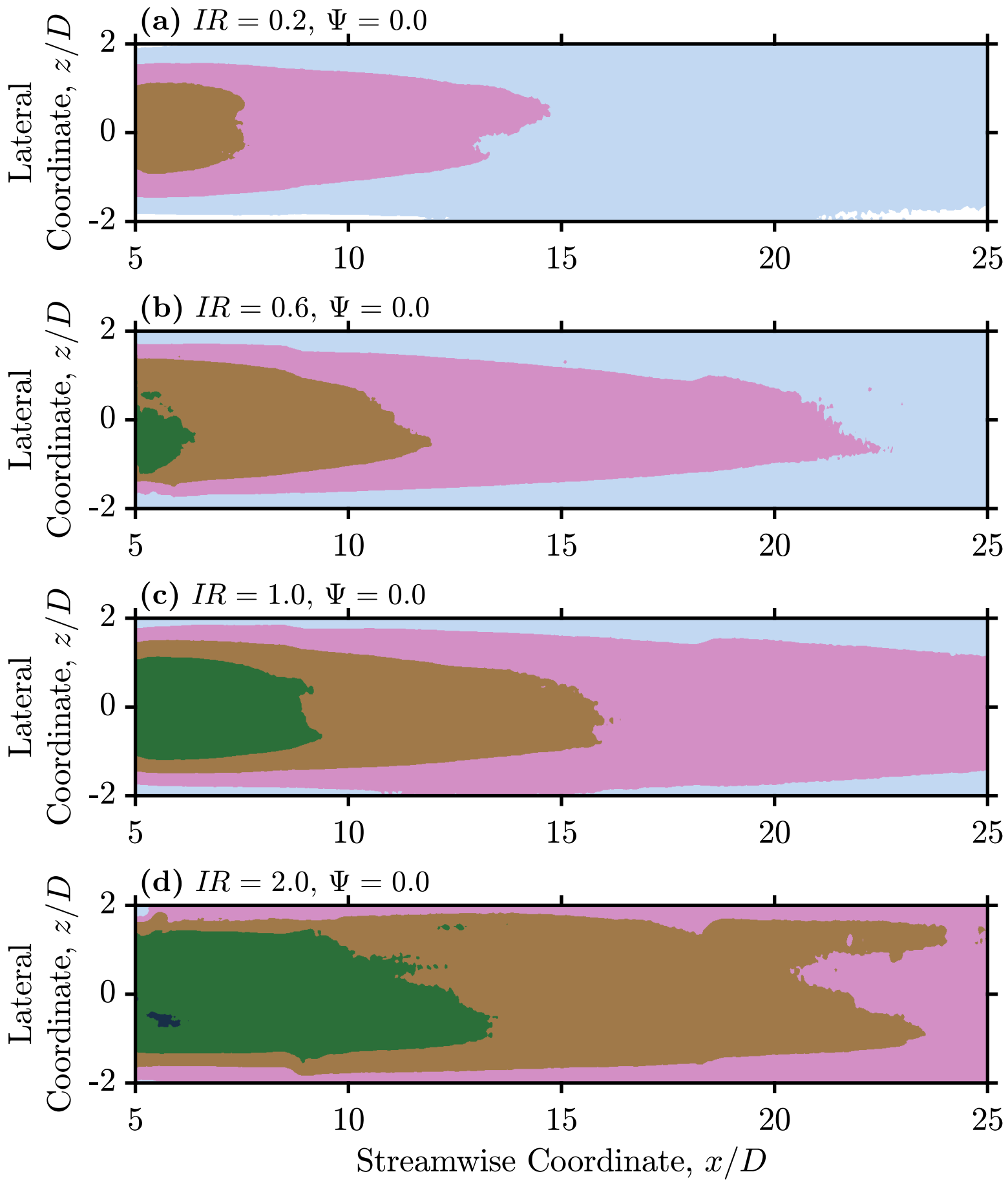

Fig. 5.7 Contours of measured film effectiveness for shaped holes under steady mainstream boundary conditions, $\Psi=0.0$, at momentum flux ratios (a) $I R=0.2$, (b) $I R=0.6,(\mathbf{c}) I R=1.0,(\mathbf{d}) I R=2.0$. 
Film Effectiveness, $\varepsilon_{\mathrm{f}}$

$\begin{array}{llll}0 & 0.2 & 0.4 & 0.6\end{array}$
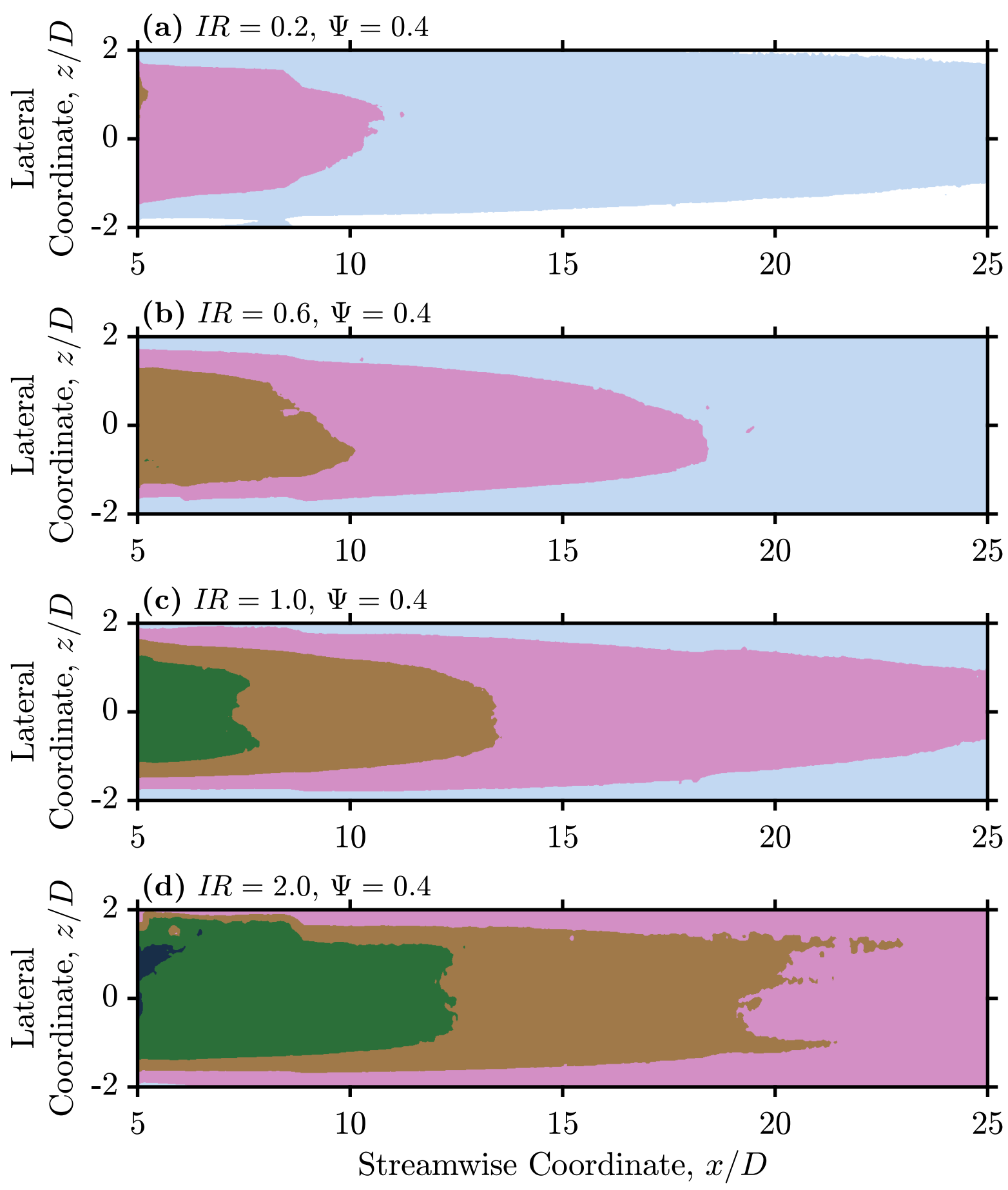

Fig. 5.8 Contours of measured film effectiveness for shaped holes under unsteady main-stream boundary conditions, $\Psi=0.4$, at momentum flux ratios (a) $I R=0.2$, (b) $I R=0.6,(\mathbf{c}) I R=1.0,(\mathrm{~d}) I R=2.0$. 


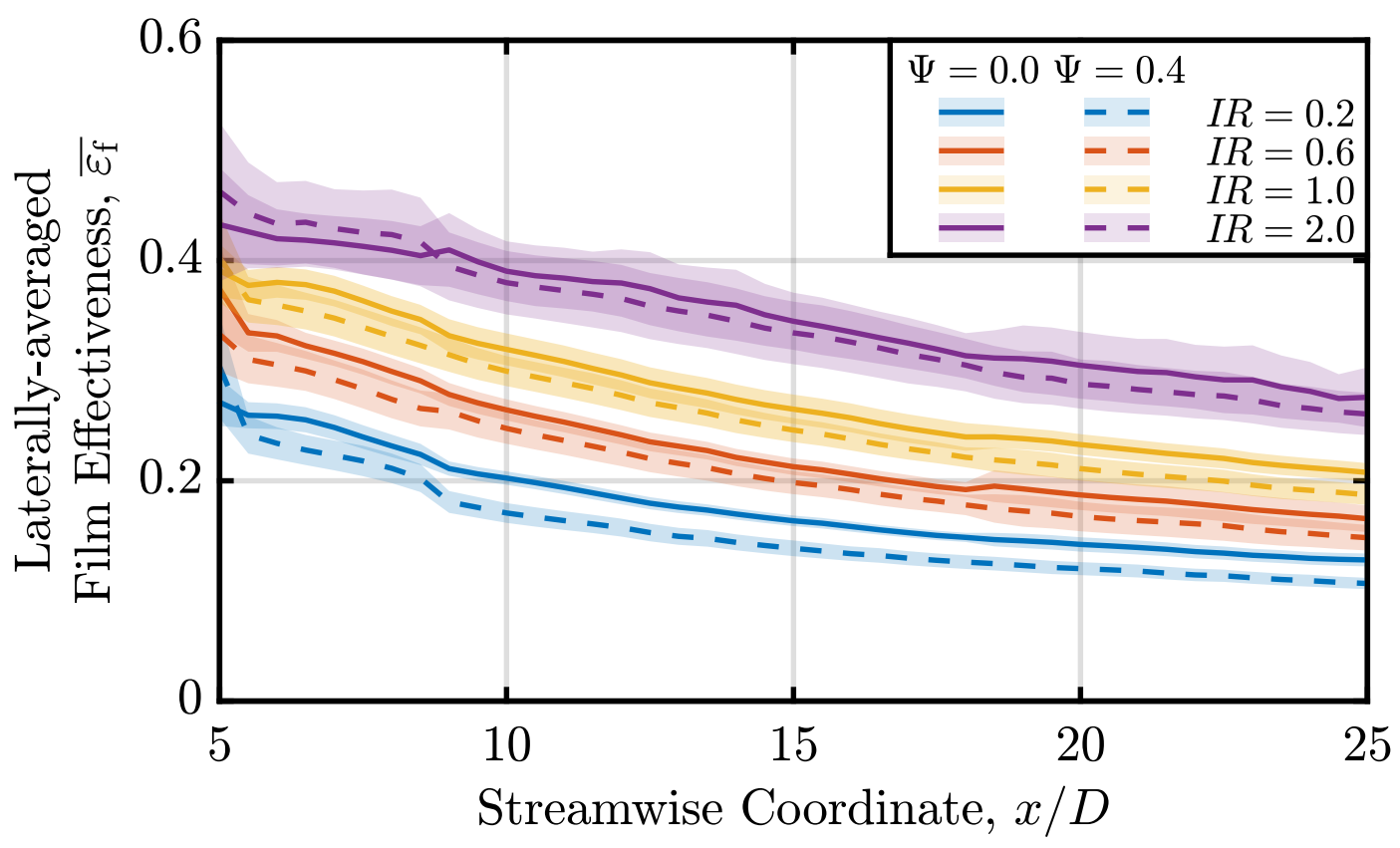

Fig. 5.9 Streamwise distributions of measured laterally-averaged film effectiveness distributions for shaped holes, with main-stream unsteadiness, $\Psi=0.4$, and without main-stream unsteadiness, $\Psi=0.0$.

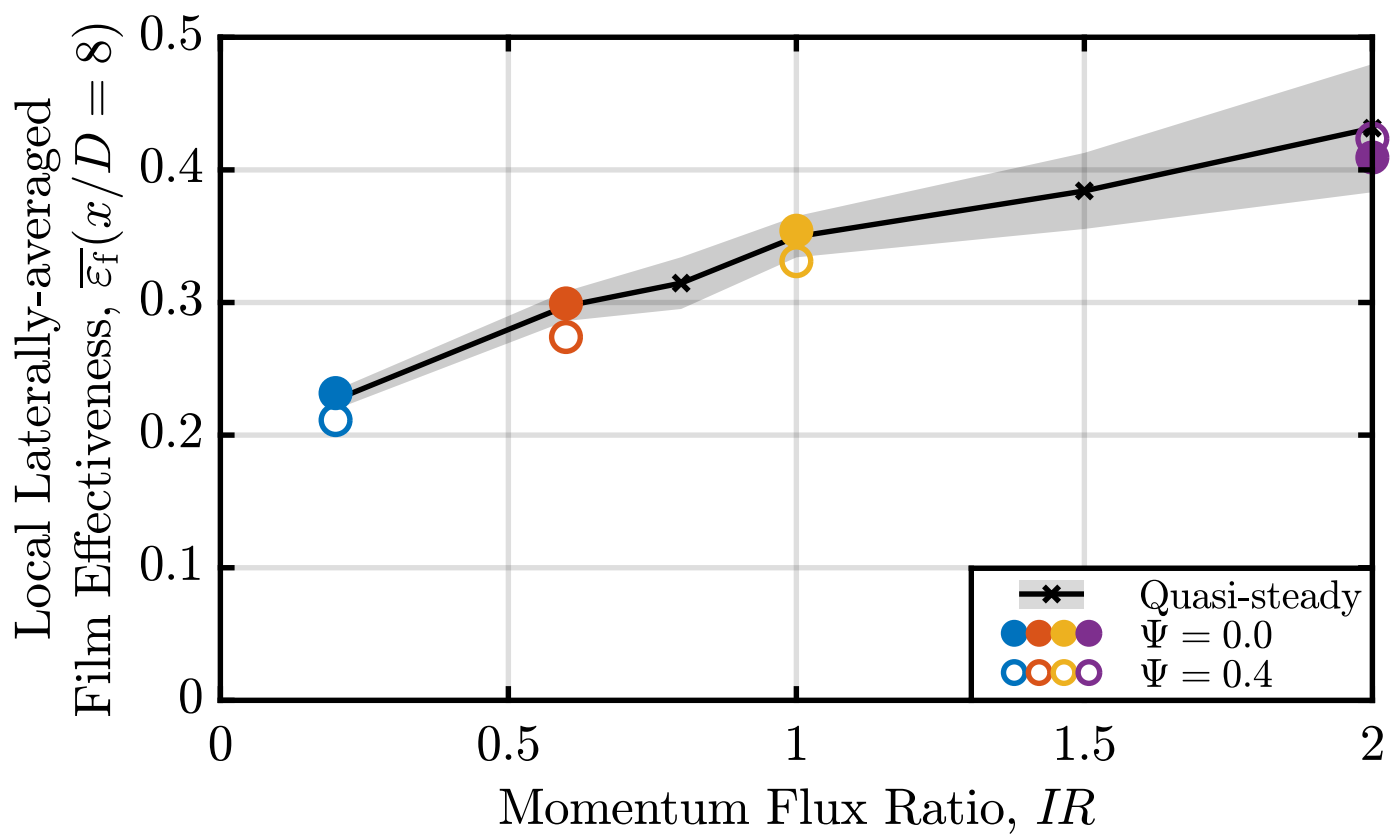

Fig. 5.10 Quasi-steady momentum flux ratio characteristic for laterally-averaged film effectiveness at $x / D=8$, shaped holes, with steady and time-averaged unsteady operating points marked. 
( $\Psi=0.0)$ and time-averaged unsteady $(\Psi=0.4)$ operating points from Figure 5.9 are shown as coloured circles. Across all tested momentum flux ratios, the characteristic is linear, which would suggest that, if the hole is behaving quasi-steadily, unsteadiness should have no effect on the time-averaged performance. Although the measured effect of unsteadiness is of similar order to the measurement uncertainty, it is a consistent drop across all momentum flux ratios.

The time scale of diffused flow within the expanded shaped hole exit is larger than the equivalent time scale of the non-diffusing cylindrical geometry. The hole area ratio takes a value $A R=1$ for the cylindrical hole, and $A R=3$ for the shaped hole. Thus, after diffusion, the local time scale and reduced frequency of the shaped hole is increased by a factor of three. It is hypothesised that unsteady perturbations to unstable separated flow in the shaped hole result in a non-quasi-steady reduction in diffusion, leading to reduced film effectiveness. The effect is smaller than changes in time-averaged momentum flux ratio, or the non-linear unsteady effect observed for the cylindrical holes at $I R=0.2$.

\subsection{Velocity field}

In this section, velocity field data are presented for cylindrical holes under steady and unsteady boundary conditions. The aim is to confirm the arguments outlined in the previous section: that the film cooling behaves quasi-steadily, and so main-stream unsteadiness only affects the time-averaged cooling performance if the hole response is non-linear.

\subsubsection{Data collection and presentation}

Hot-wire traverses are performed downstream of the cylindrical film cooling holes at a fixed streamwise location $x / D=5$. With steady boundary conditions, the velocity magnitude is time-averaged over 1000 main-stream convective time scales $D / V_{\infty}$. The output data are a set of steady velocity fields along the hole characteristic. With unsteady boundary conditions, the velocity magnitude is logged for 60 main-stream forcing periods and phase-locked ensemble averaged into 64 bins per period. The output data are phase-resolved velocity field snapshots during unsteady fluctuations, with the non-deterministic turbulent unsteadiness removed. The ensemble-averaged snapshots may be further averaged to yield the time-averaged unsteady flow field. 
Velocity magnitude data are plotted using a velocity coefficient, $C_{V}$, defined,

$$
C_{V}=\frac{V_{\mathrm{hw}} / V_{\infty}}{\left(V_{\mathrm{hw}} / V_{\infty}\right)_{y / D=2.5}}
$$

where the hot-wire velocity $V_{\mathrm{hw}}$ is first normalised by the main-stream Pitot velocity $V_{\infty}$, and then by the value of $V_{\mathrm{hw}} / V_{\infty}$ at the main-stream location $y / D=2.5$. The coefficient is formulated to capture the shape of the velocity distribution in a way that reduces the influence of drift in the tunnel operating point and hot-wire calibration.

Figure 5.11 illustrates results for the nominal operating point $I R=0.2$. The left-hand column, Figure 5.11(a), shows data for steady boundary conditions, at the nominal momentum flux ratio of $I R=0.2$ and two momentum flux ratios above and below the nominal value. The right-hand column, Figure 5.11(b), shows data for unsteady boundary conditions with time-averaged $I R=0.2$. The central plot, marked 'Avg.', is the time-averaged unsteady velocity field. The upper and lower plots, marked 'Min.' and 'Max.' respectively, are the phase-resolved snapshots corresponding to minimum and maximum unsteady excursion. Figures 5.12 and Figure 5.13 are analogous results for nominal momentum flux ratios $I R=0.6$ and $I R=1.0$.

The results allow assessment of both the quasi-steady assumption, and identification of non-linear behaviour. If the phase-resolved snapshots 'Min.' and 'Max.' are identical to a steady velocity field with the same instantaneous momentum flux ratio (comparing across the top and bottom rows), the hole is behaving quasi-steadily during its unsteady excursions. If the time-averaged unsteady velocity field 'Avg.' is not identical to a steady velocity field at the same nominal momentum flux ratio (comparing across the middle row), the hole is behaving non-linearly.

\subsubsection{Results and discussion}

Figure 5.11 shows that the velocity field of the cylindrical cooling hole responds nonlinearly to main-stream unsteadiness about $I R=0.2$. Comparing the steady result, Figure 5.11(a) $I R=0.2$, with the unsteady result, Figure 5.11(b) Avg., the shapes of the velocity contours are qualitatively different and the levels differ by up to 0.1 in velocity coefficient. This is consistent with the extant effect of unsteadiness on film effectiveness at $I R=0.2$ in Figure 5.6. The results are also suggestive of quasi-steady behaviour: comparing across the top row of Figure 5.11, the minimum phase-resolved snapshot is similar to the steady field at $I R=0.1$. Comparing across the bottom row of Figure 5.11, the steady data at $I R=0.5$ is a qualitative match for the maximum phase-resolved snapshot. 
Figure 5.12 shows velocity field data for the nominal $I R=0.6$ operating point. Comparing across the middle row, the steady and time-averaged unsteady velocity fields correspond closely, suggesting linear behaviour. This is consistent with the smaller effect of unsteadiness at $I R=0.6$, of up to 0.01 in film effectiveness from Figure 5.6. Also, comparing across the top and bottom rows, the steady velocity fields at $I R=0.5$ and $I R=0.8$ are good matches for the minimum and maximum phase-resolved snapshots respectively. Although there are local differences of order 0.05 in velocity coefficient, these should be set against the relatively larger differences of order 0.25 between operating points along the columns of the figure.

Finally, at $I R=1.0$ in Figure 5.13, similar behaviour is observed to $I R=0.6$. Good agreement is evident across all three rows of the figure, suggesting both quasi-steady and linear behaviour. This is expected from the negligible measured effect of unsteadiness on film effectiveness at this momentum flux ratio, Figure 5.5.

A more quantitative comparison of the steady and time-averaged unsteady velocity fields is presented in Figure 5.14. Here, the velocity coefficient has been laterally averaged to produce a one-dimensional wall-normal velocity profile for each of the three momentum flux ratios, with and without an unsteady main-stream. The shaded regions denote the experimental uncertainty in hot-wire velocity measurements of $U\left(V_{\mathrm{hw}} / V_{\infty}\right)= \pm 1.0 \%$ determined in Section 3.2.2. At $I R \geq 0.6$, the profiles are within the measurement uncertainty; for these operating conditions, it may be concluded that unsteadiness has no effect on the velocity profile at constant time-averaged boundary condition, and that the flow field behaves linearly. At $I R=0.2$ the time-averaged unsteady velocity magnitude is greater than the steady profile by up to 0.04 in velocity coefficient. The flow behaves non-linearly, with unsteadiness acting to increase the velocity and causing the time-averaged unsteady flow to resemble that of a higher steady momentum flux ratio. This is concordant with a reduction in effectiveness due to unsteadiness at this condition, where the time-averaged performance at $I R=0.2$ is that expected from an operating point at higher momentum flux ratio, $I R \approx 0.4$, Figure 5.6. 
(a) Steady, $\Psi=0.0$
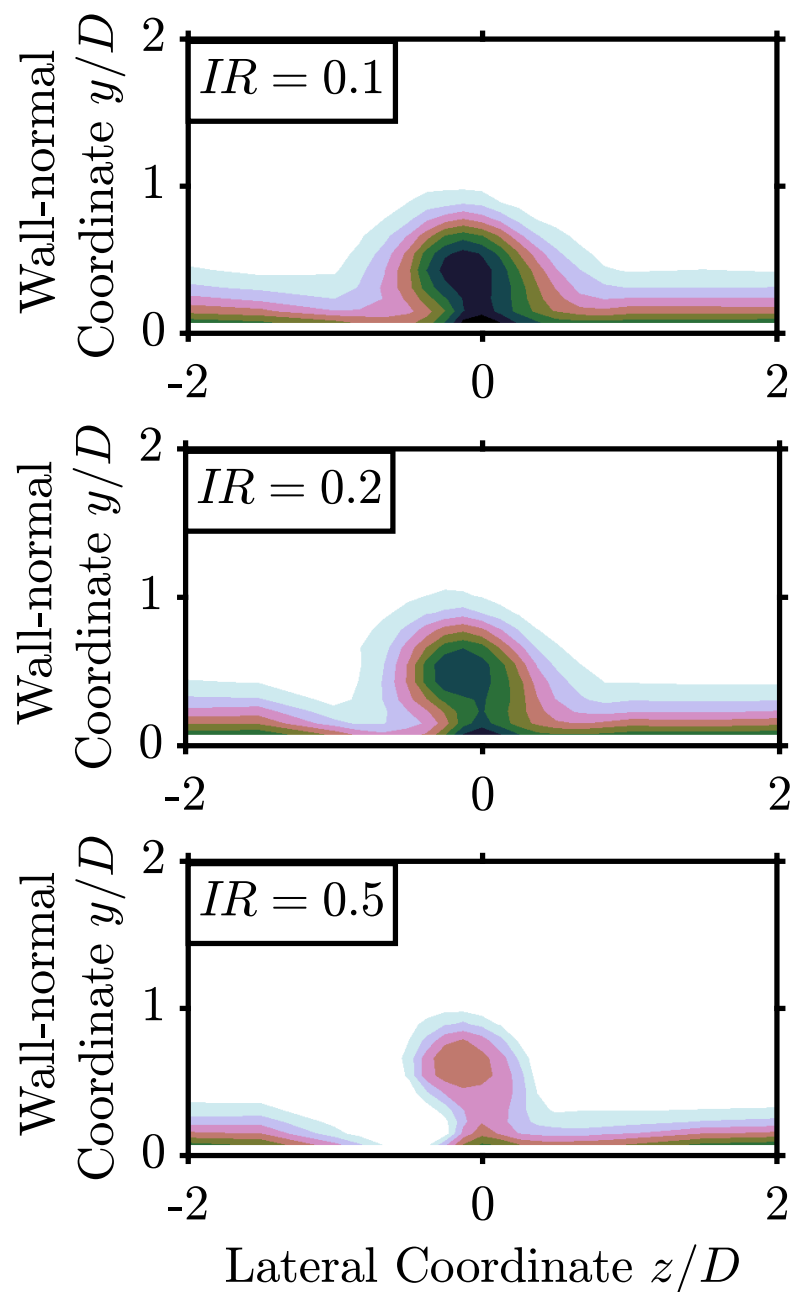

(b) Unsteady, $\Psi=0.4$

Nominal $I R=0.2$
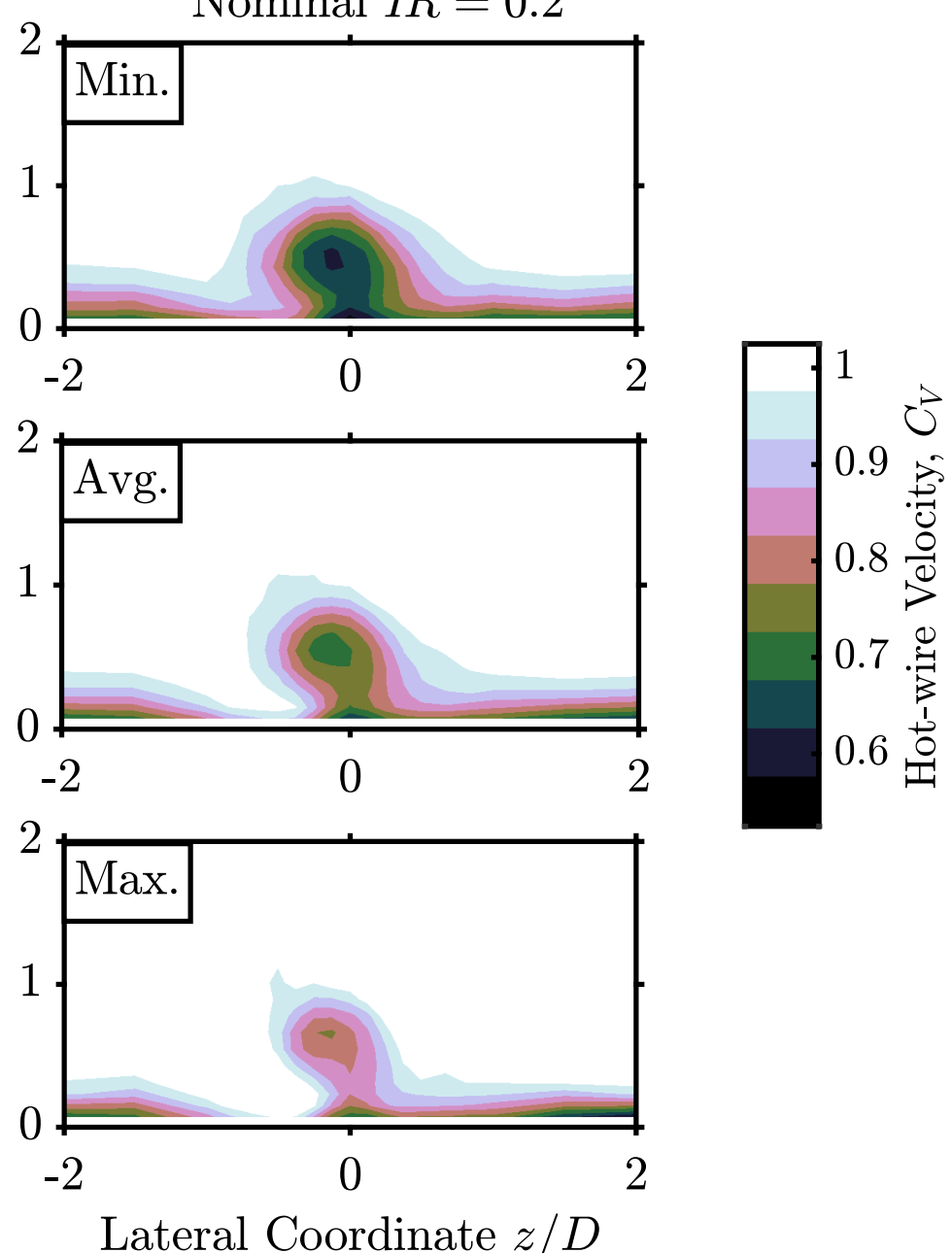

Fig. 5.11 Comparison of (a) steady, (b) time-averaged unsteady, and phase-locked ensemble-averaged velocity fields; for nominal $I R=0.2$, on $x / D=5$ traverse plane. The velocity field responds non-linearly to main-stream unsteadiness. 
(a) Steady, $\Psi=0.0$
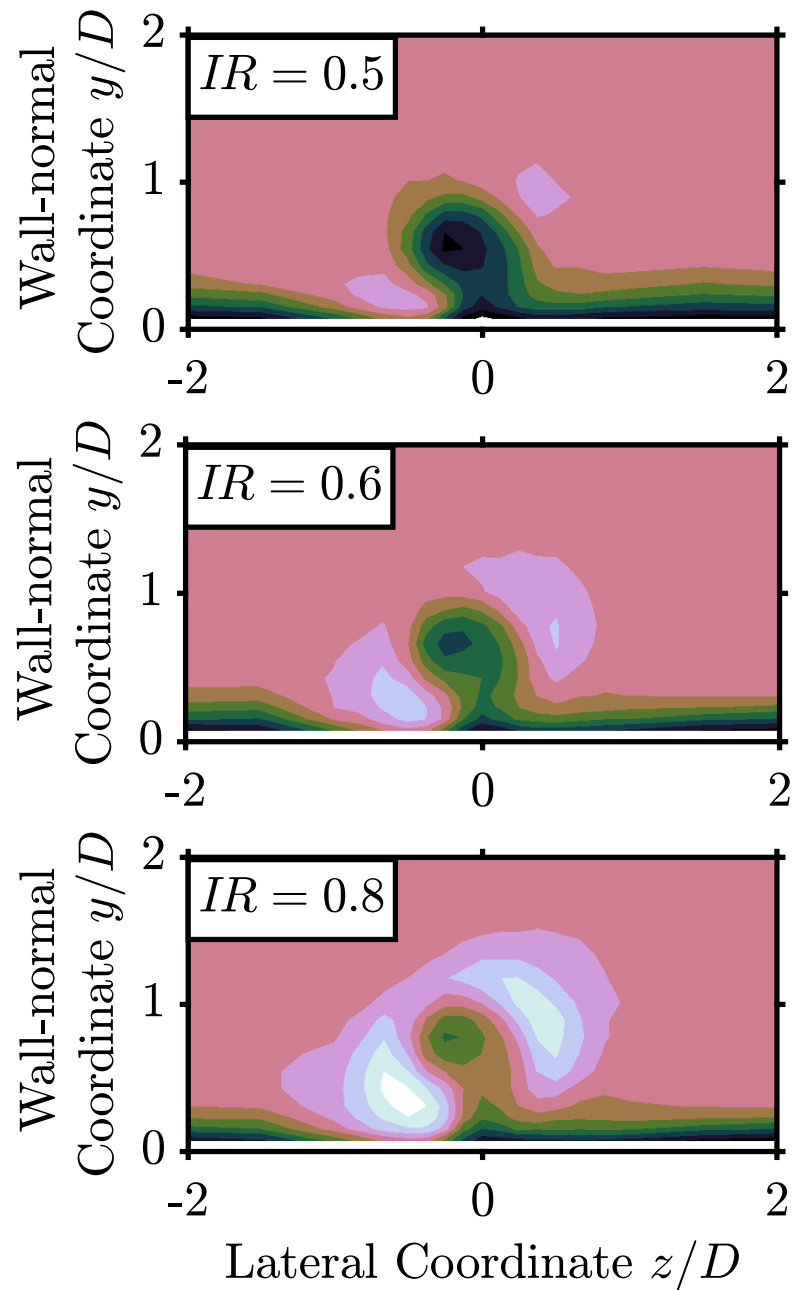

(b) Unsteady, $\Psi=0.4$

Nominal $I R=0.6$
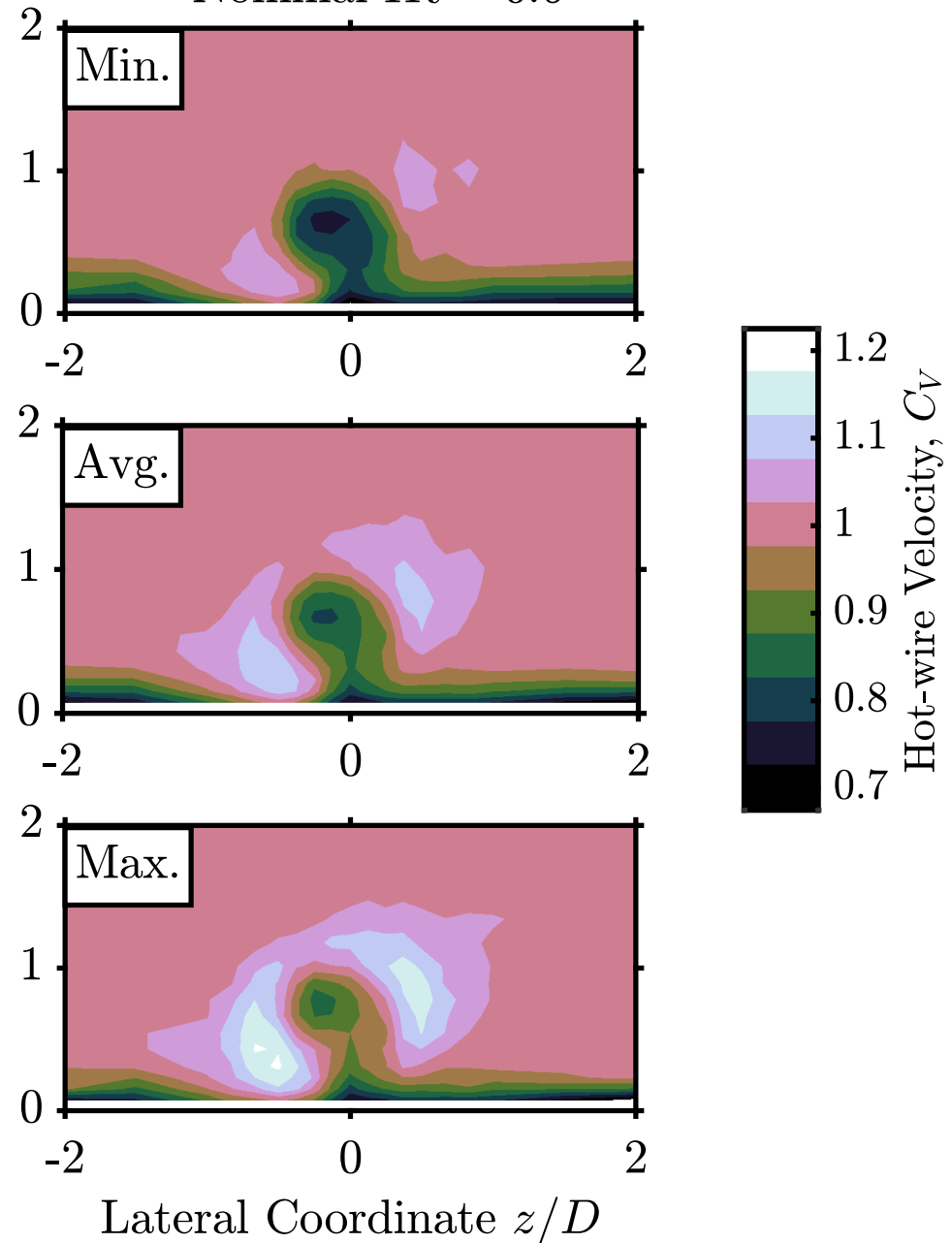

Fig. 5.12 Comparison of (a) steady, (b) time-averaged unsteady, and phase-locked ensemble-averaged velocity fields; for nominal $I R=0.6$, on $x / D=5$ traverse plane. The velocity field responds linearly to main-stream unsteadiness. 
(a) Steady, $\Psi=0.0$
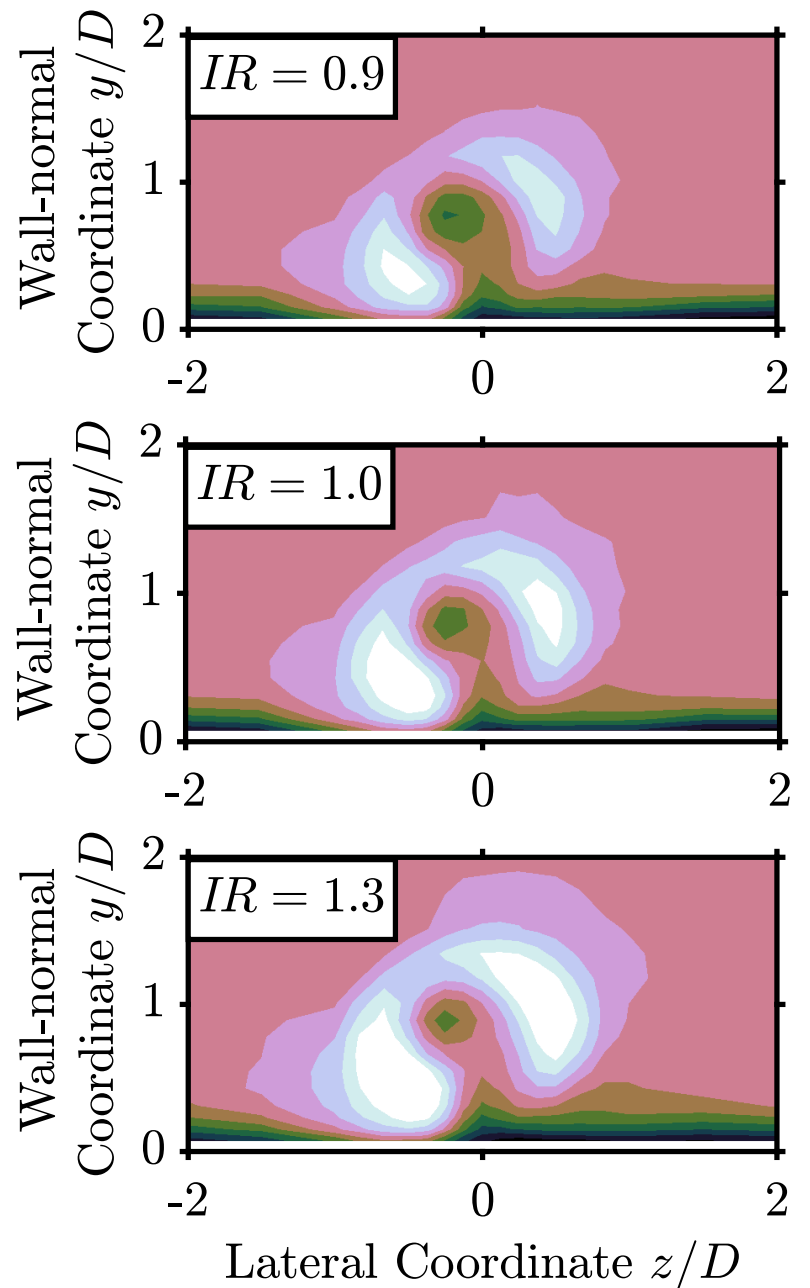

(b) Unsteady, $\Psi=0.4$

Nominal $I R=1.0$
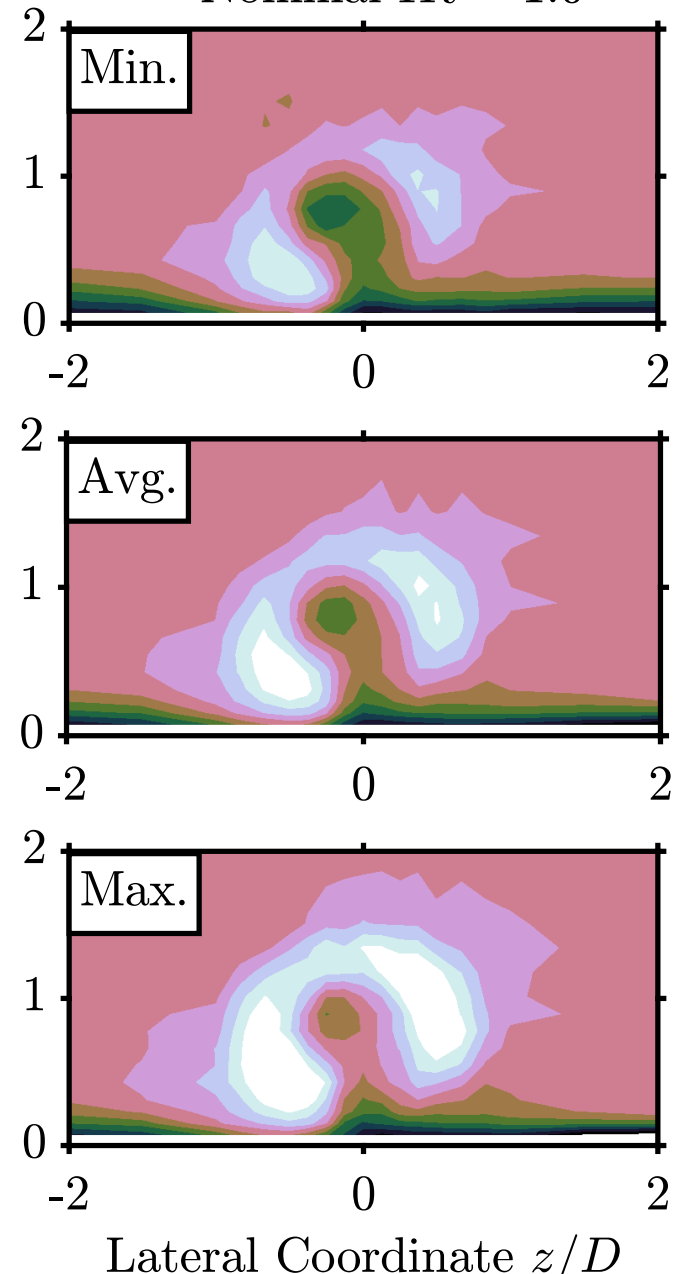

Fig. 5.13 Comparison of (a) steady, (b) time-averaged unsteady, and phase-locked ensemble-averaged velocity fields; for nominal $I R=1.0$, on $x / D=5$ traverse plane. The velocity field responds linearly to main-stream unsteadiness. 


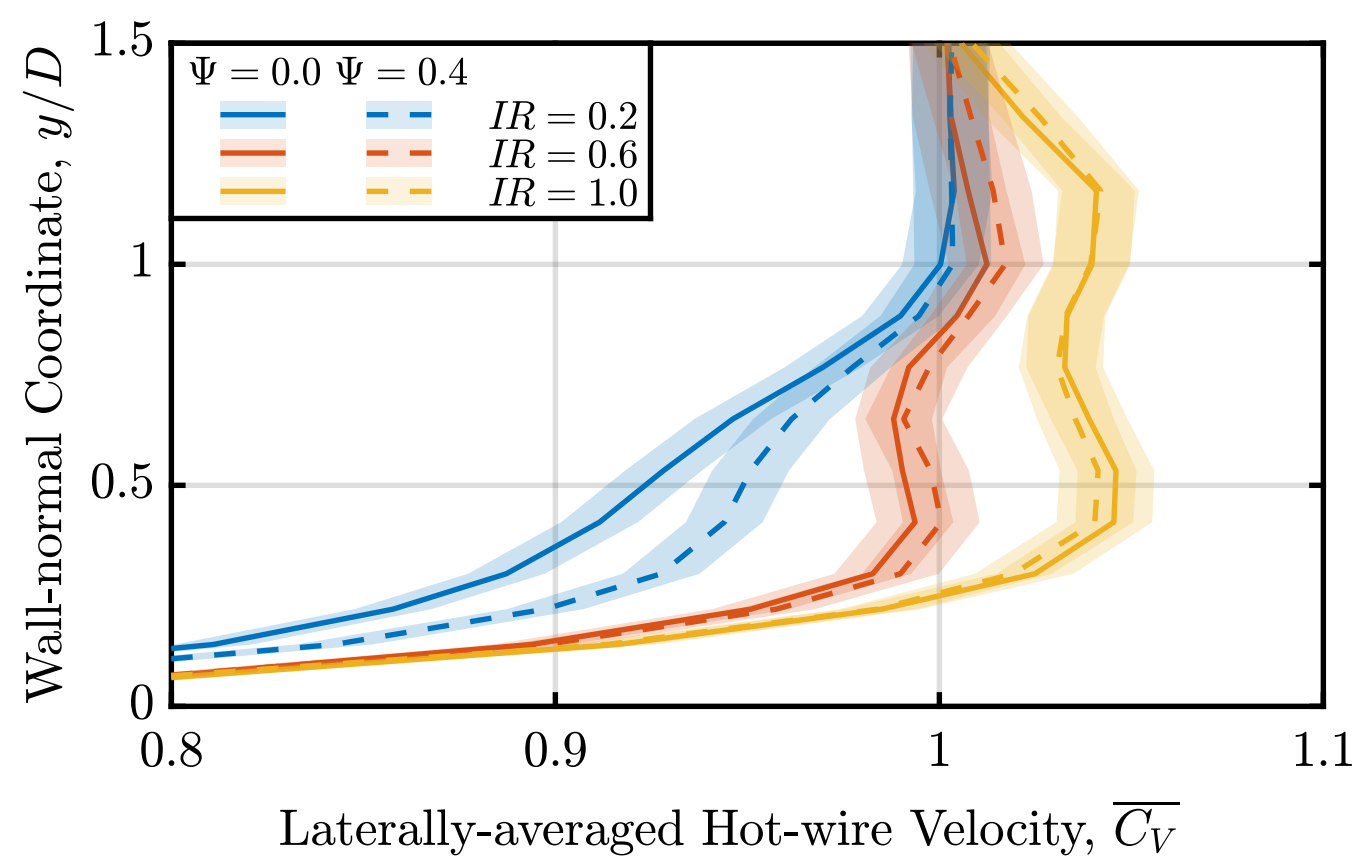

Fig. 5.14 Effect of main-stream unsteadiness on laterally-averaged hot-wire velocity profiles on $x / D=5$ traverse plane. At low momentum flux ratio, the profiles differ by up to 0.04; at high momentum flux ratio, the profiles are identical to within the measurement uncertainty. 


\subsection{Conclusions}

The aerodynamic and heat transfer measurements from the Unsteady Film Cooling Rig presented in this Chapter allow investigation of the fluid-dynamic mechanisms governing the effect of flow unsteadiness on rotor film cooling in a simplified geometry, where boundary conditions may be precisely controlled. The following conclusions are drawn from the experimental data:

1. Fast-response pressure transducer measurements show that the UFC Rig pressure fluctuator device produces an unsteady variation in cooling hole pressure drop of $0.34 \leq \Psi \leq 0.44$ main-stream dynamic heads, depending on the cooling hole geometry and flow condition. This is representative of the target amplitude $\Psi=0.4$ determined from turbine stage computations. Assuming a constant discharge coefficient, the unsteady pressure drop leads to momentum flux ratio perturbations of $0.18 \leq \widetilde{I R} \leq 0.31$

2. Measured distributions of film effectiveness for cylindrical and laid-back fan-shaped holes under steady boundary conditions are in agreement with trends reported in the literature. The film effectiveness of cylindrical holes increases with momentum flux ratio for $I R \leq 0.2$ as the coolant mass flow rate increases. Film effectiveness then reduces with momentum flux ratio for $I R \geq 0.2$ as the coolant separates from the wall. Shaped holes have higher levels of coolant coverage, compared to cylindrical holes, and do not exhibit separation from the wall, giving a monotonic rise of film effectiveness with momentum flux ratio.

3. The effect of main-stream unsteadiness on cylindrical holes varies with time-averaged momentum flux ratio. Because the reduced frequency of main-stream unsteadiness is low, the flow may be taken as quasi-steady and to follow its steady characteristic during unsteady excursions. At low momentum flux ratio, $I R=0.2$, unsteadiness reduces effectiveness by up to 0.06 or $31 \%$. This is because the hole behaves nonlinearly: at the peak of the characteristic, both positive and negative momentum flux ratio perturbations about the time-average reduce the instantaneous film effectiveness. At high momentum flux ratio, $I R \geq 0.6$, the effect of unsteadiness is smaller at \pm 0.01 in film effectiveness. This is because once coolant has separated from the wall, the hole characteristic is linear.

4. With laid-back fan-shaped holes, main-stream unsteadiness produces a consistent drop in film effectiveness of between 0.01 and 0.03 , of similar order to the measurement uncertainty. The steady shaped hole characteristic shows that film 
effectiveness increases linearly with momentum flux ratio across all operating points. Therefore, the drop in effectiveness is not explained by quasi-steady movement on the characteristic. It is hypothesised that the diffusing and separated flow within the shaped hole has a longer characteristic time scale, and unsteady perturbations result in a non-quasi-steady reduction in performance. The effect is smaller than the quasi-steady, non-linear reduction in film effectiveness for cylindrical holes in point 3

5. Hot-wire measurements of cylindrical hole velocity fields under steady and unsteady boundary conditions confirm the arguments of point 3. At all momentum flux ratios, phase-locked ensemble-averaged snapshots of the velocity field with main-stream unsteadiness correspond closely to data with steady boundary conditions at the instantaneous momentum flux ratio, suggesting that the flow is behaving quasisteadily. If the velocity fields are laterally-averaged to produce one-dimensional wall-normal profiles, the steady and time-averaged unsteady data for $I R \geq 0.6$ match to within the measurement uncertainty, indicating linear behaviour. The time-averaged unsteady velocity profile at $I R=0.2$ differs with the steady velocity profile by up to 0.04 laterally-averaged velocity coefficient, indicating non-linear behaviour. 



\title{
Chapter 6
}

\section{Non-film-resolved blade row interaction simulations}

\begin{abstract}
The mechanisms of blade row interaction affecting rotor film cooling are identified, in order to make recommendations for cooling design in the real, unsteady turbine environment. URANS computations are used to predict unsteady aerodynamics in a turbine stage, and a reduced-order model to predict the consequent response of rotor cooling holes.

The upstream vane wake creates a 'negative-jet' perturbation in the rotorrelative frame, causing migration of vane coolant towards the rotor suction side, and producing main-stream velocity perturbations of $\pm 15 \%$ isentropic rotor exit velocity. Vane potential field interaction creates pressure perturbations of $\pm 10 \%$ isentropic rotor exit dynamic head moving circumferentially in the rotor-relative frame, which propagate as approximately one-dimensional waves through the rotor passage.

A quasi-steady hole response model is used to predict instantaneous unsteady momentum flux ratio of rotor cooling holes; fluctuations of at least $\pm 30 \%$ are observed. Computations with modified upstream vanes are used to vary the relative strength of wake and potential field interaction. In general, both mechanisms contribute to rotor film cooling unsteadiness.

It is recommended that the designer should choose a cooling configuration which behaves linearly over the expected unsteady excursions in operating point, which may be found using a quasi-steady model.
\end{abstract}




\subsection{Introduction}

In this Chapter, the blade row interaction mechanisms causing unsteady main-stream boundary conditions in a turbine rotor are characterised, and the resultant excursions in rotor cooling hole momentum flux ratio are quantified. It is shown in Chapter 5 that unsteadiness in momentum flux ratio will affect time-averaged film cooling performance if the fluctuations occur over a non-linear region of the hole characteristic. Therefore, prediction of the range of unsteady excursions is of interest.

The structure of this Chapter is as follows. First, the conceptual approach for modelling the unsteady hole response is described. Next, blade row interaction mechanisms are identified and illustrated using non-film-resolved computational results. Then, the hole response model is used to predict instantaneous unsteady momentum flux ratio due to the different interaction mechanisms. Finally, the results are used to make recommendations for the design of film cooling in the unsteady turbine environment.

\subsection{Conceptual approach}

A reduced-order modelling approach to assess the effects of blade row interaction on rotor film cooling is now outlined. The diagram of Figure 6.1 illustrates the components of the modelling framework. The central assumption is that the hole flow behaves quasi-steadily during unsteady interactions, because the reduced frequency is low, i.e. the time scale of the hole flow is much less than the vane passing period. The definition of reduced frequency and the quasi-steady assumption are discussed in more detail in Section 2.4.1for a large industrial gas turbine, the reduced frequency is of order $\kappa_{\mathrm{c}} \approx 0.06$, a low value, which supports the assumption.

Taking the hole flow as quasi-steady simplifies modelling, as existing steady-state hole characterisations can be used separately at each instant in time. However, timevarying unsteady boundary conditions are still required. The computations used to provide instantaneous main-stream boundary conditions, the choice of internal boundary conditions, and the quasi-steady hole characterisation itself are now described.

\subsubsection{Non-film-resolved computations}

Film cooling holes do not need to be resolved to predict blade row interactions. Therefore, non-film-resolved computations are suitable for modelling blade row interaction and the associated unsteady main-stream boundary conditions on rotor cooling holes. Modelling 


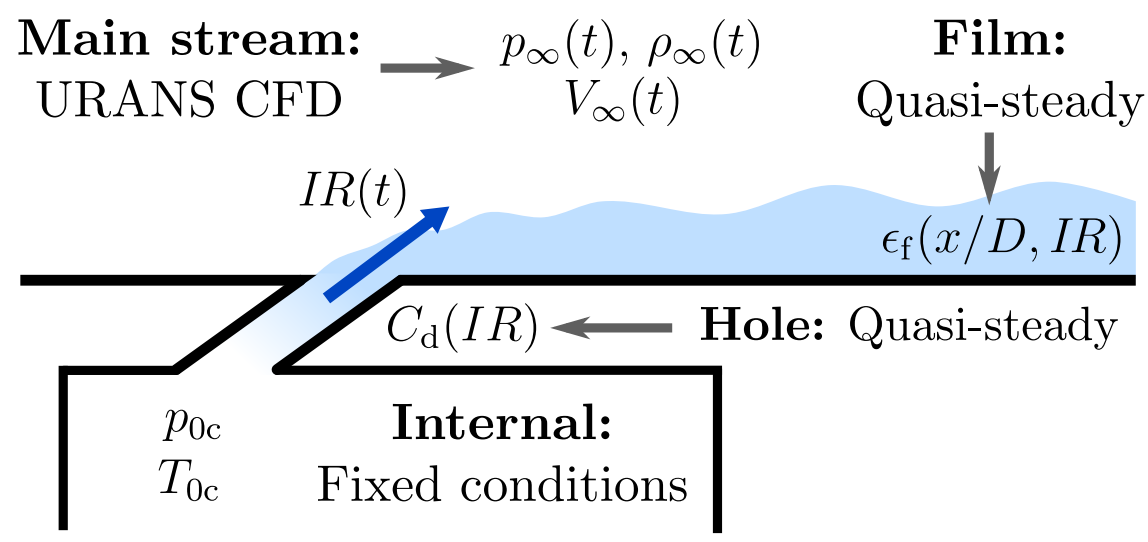

Fig. 6.1 Diagram of the unsteady film cooling reduced-order modelling approach. Internal boundary conditions are fixed, main-stream boundary conditions are extracted from URANS computations, and the cooling hole is assumed to behave quasi-steadily.

the film cooling without resolving individual cooling holes reduces computational cost by two orders of magnitude.

In this Chapter, results from URANS computations are presented. The case analysed is representative of a large industrial gas turbine. The computational domain includes the first one-and-a-half stages of the high-pressure turbine. Film cooling is treated using surface patches with additional fluxes of mass, momentum and energy. This simplified model is not expected to give realistic surface temperature distributions, but captures the effects of coolant addition on the turbine aerodynamics. The resulting surface temperature distributions are not used. Full details of the non-film-resolved computational approach are given in Section 4.2.

Instantaneous main-stream boundary conditions, namely static pressure, density and velocity, are extracted from the simulations and supplied as input to the quasi-steady hole response model. The main-stream is taken as a line offset by $5 \%$ of blade pitch away from the blade surface, outside of the coolant films.

\subsubsection{Internal boundary conditions}

Previous studies on unsteady film cooling have assumed, without a stated justification, that the internal boundary condition on the cooling hole is a constant plenum pressure (Abhari and Epstein, 1994). However, during commissioning of the experimental apparatus described in Chapter 3, oscillations in plenum pressure were identified which prompted a re-examination of the validity of this assumption in the full-scale turbine. 
In a real machine, the blade coolant supply pressure is set by the total mass flow of coolant and total loss through the secondary air system (Hodson, 2017). Each cooling hole makes only a small contribution to these totals, so that the blade coolant supply pressure may be taken as independent of the individual hole mass flow rates. In the laboratory experiment, however, flow through the test holes is the only contribution to flow through the coolant blower. Unsteady variations in hole mass flow therefore alter the blower operating point, causing unrealistic oscillations in plenum pressure. The oscillations were eliminated by installing a large plenum downstream of the coolant blower, in order to keep the blower operating point constant.

In summary, plenum pressure oscillations do not occur in the real machine because the coolant supply pressure is insensitive to hole mass flow rate. Therefore, the plenum pressure is set to a constant value, selected to give a pressure margin of $3 \%$ with respect to the mass-averaged rotor inlet relative stagnation pressure. The coolant temperature is set to yield a stagnation temperature ratio of 0.5 with respect to the mass-averaged rotor inlet relative stagnation temperature.

\subsubsection{Unsteady hole response model}

The cooling hole operating point is fixed by the combination of momentum flux ratio, $I R$, and density ratio, $D R$. The momentum flux ratio requires evaluation of the hole mass flow rate, for which the pressure loss through the hole must be determined. This loss is characterised using a discharge coefficient, $C_{\mathrm{d}}$, defined as the ratio of actual mass flow to ideal mass flow assuming a one-dimensional isentropic expansion from the coolant stagnation pressure to the main-stream static pressure, for compressible flow,

$$
C_{\mathrm{d}}=\frac{\dot{m}_{\mathrm{c}}}{p_{0 \mathrm{c}} A_{\mathrm{c}}\left(\frac{p_{\infty}}{p_{0 \mathrm{c}}}\right)^{\frac{(\gamma+1)}{2 \gamma}}\left[\frac{2 \gamma}{(\gamma-1) R T_{0 \mathrm{c}}}\left(\left(\frac{p_{0 \mathrm{c}}}{p_{\infty}}\right)^{\frac{\gamma-1}{\gamma}}-1\right)\right]^{1 / 2}}
$$

where $\gamma$ is the ratio of specific heats, and $R$ the specific gas constant.

The discharge coefficient, a non-dimensional aerodynamic parameter, is a function of the non-dimensional flow conditions. Rowbury et al. (2001) presented a correlation for the discharge coefficient of inclined cylindrical holes in terms of momentum flux ratio and Reynolds number, under steady conditions. If the hole flow is quasi-steady, as assumed in the present model, then the instantaneous discharge coefficient at any point in time is given by evaluating the correlation at the instantaneous momentum flux ratio. An iterative calculation is required because the momentum flux ratio is itself a function of hole mass flow rate and hence discharge coefficient. For normal-angled holes 
on the leading-edge showerhead, no correlation data is available, so these are assumed to have a constant discharge coefficient of $C_{\mathrm{d}}=0.75$; based on the small effect of discharge coefficient non-linearity demonstrated in Section 6.4.2, this is considered an acceptable simplification.

A further (unvalidated) assumption, that steady-state film effectiveness correlations can also be applied in the unsteady flow, would furnish instantaneous cooling performance. However, the design recommendations made here are justified on the measurements of Chapter 5 and non-film-resolved computations. No attempt is made to evaluate unsteady film effectiveness in the low-order framework, because of the extra assumptions involved. High-fidelity modelling is used in Chapter 8 to predict instantaneous rotor film effectiveness subject to blade row interaction.

\subsection{Mechanisms of blade row interaction}

In this section, results from URANS computations are used to identify and illustrate the blade row interaction mechanisms generating unsteady flow in a high-pressure turbine stage. If the hole response is non-linear, main-stream unsteadiness will alter the timeaveraged cooling performance, as shown in Chapter 5. All analysis takes place on a mid-span streamsurface, removing the effects of three-dimensional secondary flow. Wake and potential field interactions are discussed in turn.

\subsubsection{Wake interaction}

\section{Negative-jet perturbation}

The wake 'negative jet' is a general blade row interaction mechanism previously reported in the Turbomachinery literature, as discussed in Section 2.2. The specific nature of the negative jet in the present case is illustrated in Figure 6.2. Perturbation values for flow properties are defined as the instantaneous minus the time-averaged values, denoted using a prime. The contours in Figure 6.2 are the relative velocity magnitude perturbation at a particular instant in time on the rotor mid-span streamsurface, normalised by the isentropic exit velocity reference. Vectors are also plotted to indicate the associated perturbation direction.

The flow structure is similar to a classical negative jet, shown in Figure 2.2. Upstream of the rotor, within the wake, perturbation vectors act down and to the left of the frame, opposing the time-averaged flow direction and indicating a velocity deficit. Within the passage, the convecting wake produces a cross-stream, vortex-pair-like perturbation. At 

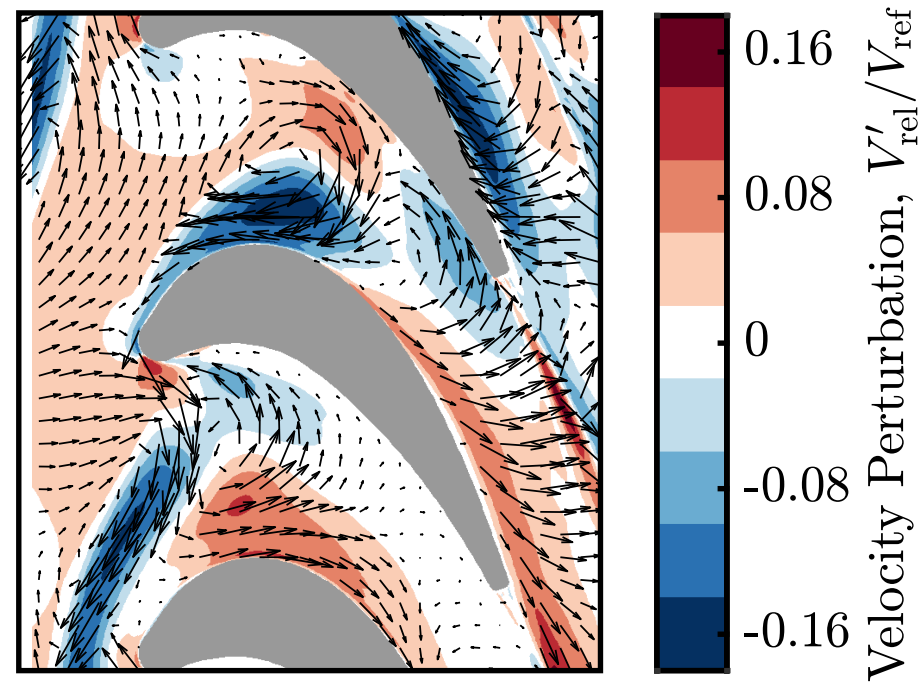

Fig. 6.2 Instantaneous relative velocity magnitude perturbation, with arrows indicating the perturbation vector, at rotor mid-span. Velocity normalised by isentropic exit relative velocity. A negative jet acts towards the suction side, perturbing the main-stream velocity by up to $\pm 15 \%$ reference velocity.

the instant shown, the negative jet is acting to reduce the main-stream velocity on the suction side, by $15 \%$ of the reference value. This affects instantaneous hole operating point via the denominator of momentum flux ratio.

\section{Stagnation point oscillation}

As the rotor rotates through the non-uniform flow angle leaving the upstream stator, the blade leading-edge stagnation point oscillates due to changing incidence. As identified by Johnson et al. (2009), this has implications for the showerhead cooling performance. For a single hole on a cylindrical leading edge, they measured a reduction in overall effectiveness of $25 \%$ due to an incidence oscillation of $\pm 5^{\circ}$, compared to a steady zero-incidence flow. As these situations have the same time-averaged boundary conditions, the film cooling is behaving non-linearly. The non-linearity arises from a discontinuity in response when the stagnation point crosses a cooling hole, as coolant is exclusively directed to either the pressure or suction side.

Two instantaneous snapshots of the rotor mid-span leading edge flow field are shown in Figure 6.3. The static pressure contours indicate the stagnation point position, and insets show the wake location identified using a temperature perturbation line contour. Static pressure is plotted as a pressure coefficient, $C_{p}$, with respect to isentropic rotor 

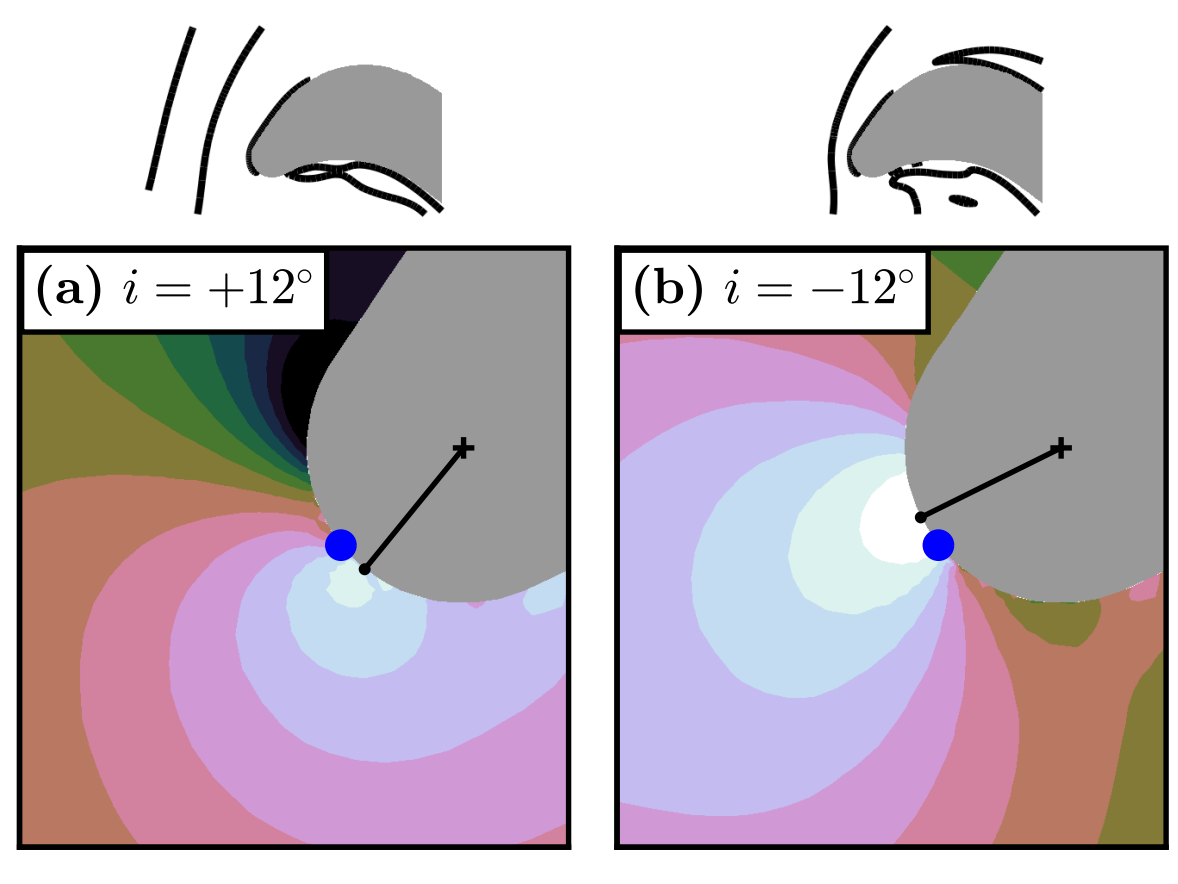

\section{Wake Location}

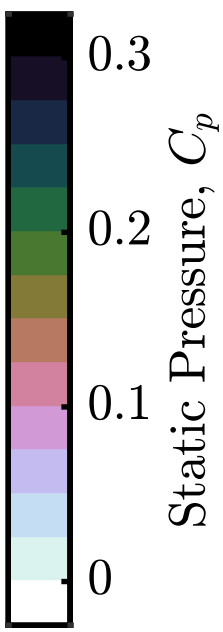

Fig. 6.3 Instantaneous static pressure fields at rotor mid-span leading edge: (a) maximum incidence, outside wake; (b) minimum incidence, during wake impact. The stagnation point is marked in black, and a nominal showerhead cooling hole location in blue. The stagnation point oscillates by $\pm 12^{\circ}$, crossing the cooling hole during a vane passing cycle.

exit conditions,

$$
C_{p}=\frac{p_{0 \mathrm{ref}}^{\mathrm{rel}}-p}{p_{0 \mathrm{ref}}^{\mathrm{rel}}-p_{\mathrm{ref}}} .
$$

Incidence, $i$, is measured on the leading-edge circle from a showerhead cooling hole positioned on the time-averaged stagnation point, marked in blue. The maximum incidence $i=+12^{\circ}$ in Figure 6.3(a) corresponds to an instant where the wake is remote from the leading edge, and showerhead coolant flows onto the suction side. When the low-velocity wake impacts the rotor leading edge, Figure 6.3(b), the incidence reduces to $i=-12^{\circ}$ and coolant flows onto the pressure side.

Figure 6.3 confirms the existence of incidence fluctuations in a real turbine. However, the amplitude of incidence oscillation in the present case is twice that estimated by Johnson et al. (2009), who used vane exit velocity triangle arguments with a nominal wake velocity deficit. A similar analysis for the present turbine predicts the expected incidence oscillation to within $1^{\circ}$. This suggests that stagnation point movement can be directly inferred from the vane exit flow angle non-uniformity. 
(a) Steady

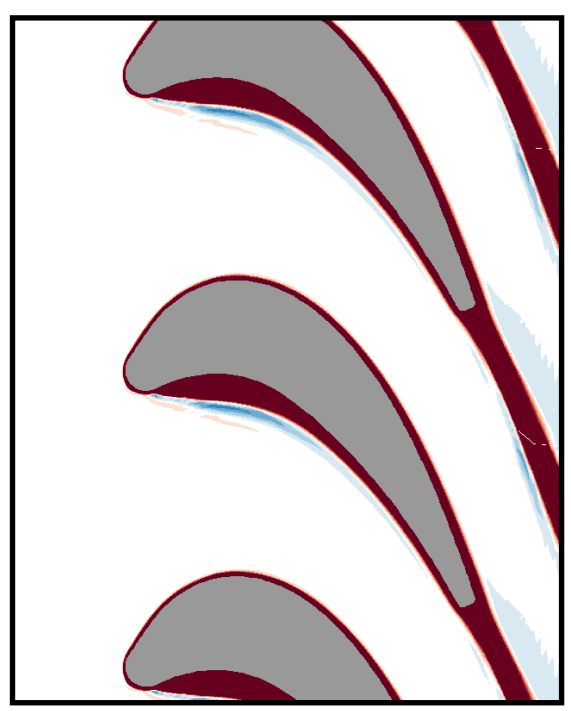

(b) Unsteady

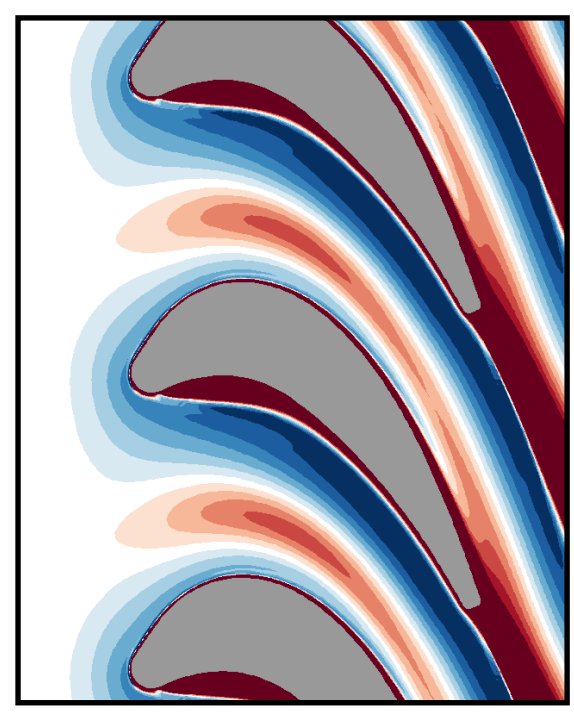

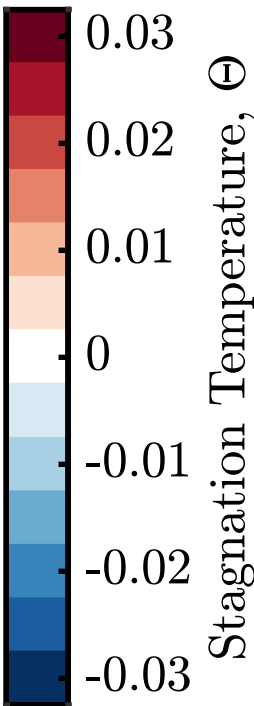

Fig. 6.4 Time-averaged rotor-relative stagnation temperature at rotor mid-span, plotted as effectiveness referenced to mass-averaged rotor inlet and blade coolant temperatures: (a) steady, and (b) unsteady computations. Blade-row interaction creates non-uniformity in main-stream temperature equivalent to \pm 0.025 effectiveness.

\section{Vane coolant migration}

With cooled upstream vanes, wake interaction affects the time-averaged temperature field within the rotor. This is a kinematic effect, where cold fluid within the wake is carried across the blade passage by the negative jet perturbation. The effect of this transport is quantified using contours of relative stagnation temperature, $T_{0}^{\text {rel }}$, time-averaged in the rotating frame, at rotor mid-span in Figure 6.4. Temperature is plotted as an effectiveness, $\Theta$, with respect to the mass-averaged rotor inlet temperature and blade coolant temperatures,

$$
\Theta=\frac{T_{0 \infty}^{\mathrm{rel}}-T_{0}^{\mathrm{rel}}}{T_{0 \infty}^{\mathrm{rel}}-T_{0 \mathrm{c}}^{\mathrm{rel}}}
$$

so that $\Theta=0$ corresponds to the main-stream temperature in a mixing-plane simulation, and on the rotor surface $\Theta=\varepsilon_{\mathrm{f}}$.

As a reference case, results from a steady computation where the wake is mixed out before entering the rotor are shown in Figure 6.4(a). Coolant films can be identified as red areas around the blade, and away from the films $\Theta=0$ as expected. In an unsteady computation, Figure 6.4(b), the temperature field is non-uniform. Areas of positive 
effectiveness near the suction side, and negative effectiveness near the pressure side, are in accordance with cold wake fluid migrating with the negative jet across the passage. However, close to the suction side, there is another smaller area of negative effectiveness, which indicates that the full kinematics are more complex than a one-dimensional view.

The redistribution of vane coolant by wake interaction causes variations in timeaveraged main-stream temperature equivalent to \pm 0.03 in blade film effectiveness. This is $\pm 8 \%$ of a typical design target of $\varepsilon_{\mathrm{f}} \approx 0.4$. Previous rotating-stage experiments with cooled vanes (Abhari, 1996; Abhari and Epstein, 1994) have found trends of reduced pressure-side effectiveness, and no change or an increase in suction-side effectiveness, consistent with the direction of temperature redistribution observed here.

\section{Main-stream turbulence}

The vane wake periodically increases main-stream turbulence intensity as it convects through the rotor. For a constant momentum flux ratio, the effect of time-varying wake turbulence may be adequately modelled using just a time-averaged turbulence intensity if the film effectiveness responds linearly to a change in turbulence intensity. From the literature reviewed in Section 2.4.2, it is known that the effects of (unsteady) wakes and (steady) elevated main-stream turbulence intensity are similar. This suggests that the response to wake turbulence is indeed linear - the effect of wake turbulence is investigated further using film-resolved computations in Chapter 8.

\subsubsection{Potential field interaction}

\section{Frozen potential field}

The potential fields of the upstream and downstream vanes impose static pressure distortions at the rotor inlet and exit, rotating at blade speed in the relative frame. Four snapshots of the instantaneous static pressure perturbation at rotor mid-span during the upstream vane passing cycle are shown in Figure 6.5. The pressure perturbation is non-dimensionalised to a pressure coefficient using the reference dynamic head as in Equation (6.2). The boundary of the upstream vane wake is shown using a line contour of temperature perturbation.

The upstream vane potential field is the circumferential distortion of $\pm 10 \%$ reference dynamic head moving downwards at the left edge of the frame, with wavelength 2.4 rotor pitches. The potential field of the downstream vane, wavelength 1.5 rotor pitches, is less distinctive, with small perturbations of up to $\pm 3 \%$ reference dynamic head at the right edge of the frame. This is because the pitch of the downstream vane is $63 \%$ that of the 

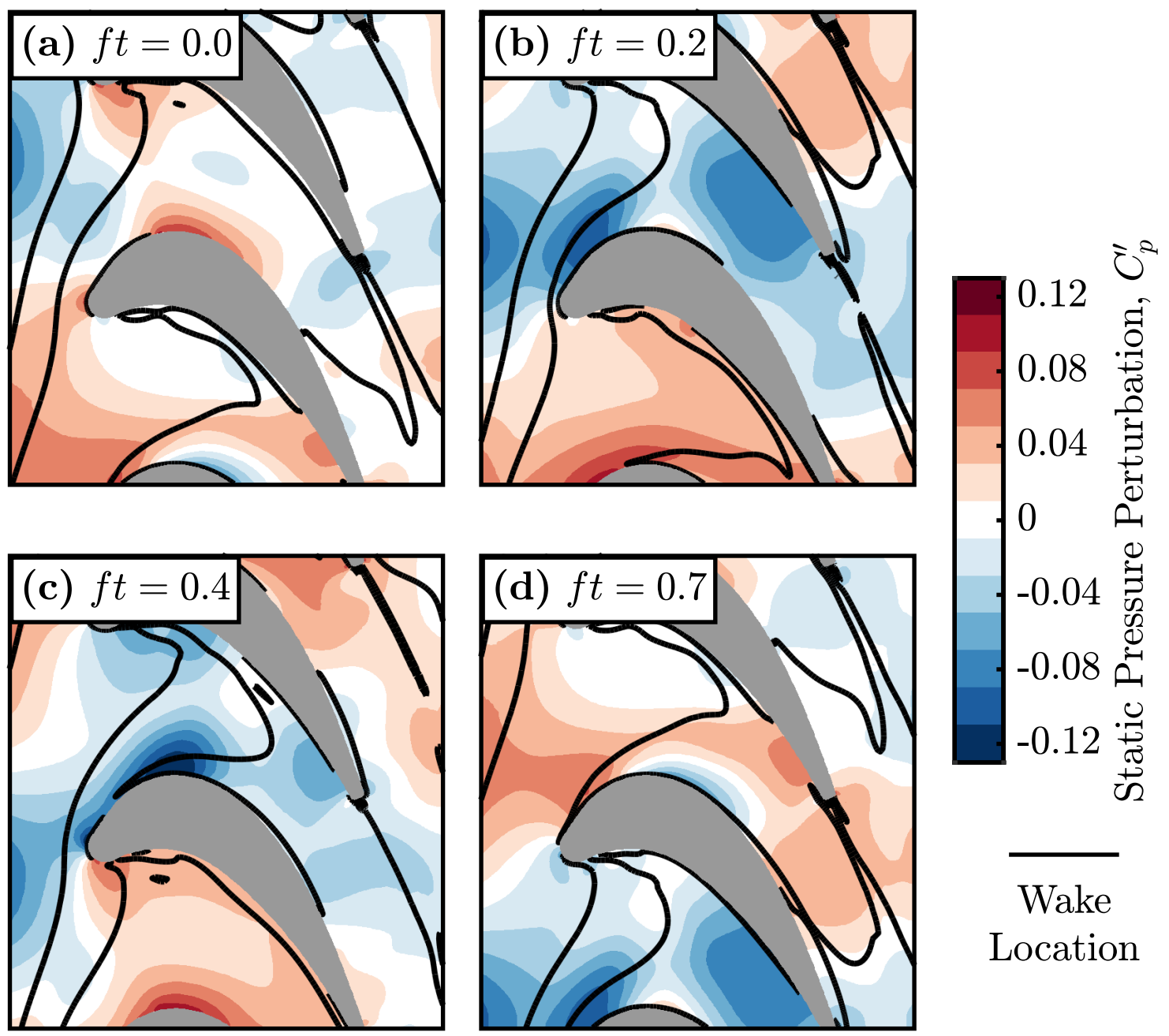

\section{Wake \\ Location}

Fig. 6.5 Static pressure perturbation contours at rotor mid-span for four instants during upstream vane passing. Pressure coefficient referenced to isentropic exit conditions; wake location marked with black line contour of temperature perturbation. The rotor main-stream static pressure fluctuates by $\pm 10 \%$ reference dynamic head. 
upstream vane, so in this case the downstream potential field decays to a negligible level before reaching the rotor.

The upstream vane potential field is imposed directly on the rotor leading edge, where the associated main-stream static pressure fluctuations will drive showerhead cooling unsteadiness. However, there are also pressure fluctuations present at locations throughout the rotor passage, where the potential field of an isolated upstream vane would have decayed away. For example: up to $+10 \%$ reference dynamic head on the rear pressure side at $f t=0.2$, Figure $6.5(\mathrm{~b})$, and up to $+13 \%$ reference dynamic head on the suction side at $f t=0.4$, Figure $6.5(\mathrm{c})$. These fluctuations are generated via different mechanisms that are discussed in the following two sections.

\section{One-dimensional pressure waves}

The static pressure distortion due to to the upstream vane does not decay exponentially with axial distance on interacting with the rotor, as a potential field would do. Instead, pressure unsteadiness in the rotor passage is due to propagation of one-dimensional waves, first identified by Kachel and Denton (2006). One-dimensional pressure waves were concluded to be the dominant cause of static pressure fluctuations on the rotor pressure side in their model multi-stage turbine. A simple one-dimensional model has been formulated to examine the validity of this hypothesis in the present case.

The one-dimensional, compressible, unsteady Euler equations are solved over a straight contracting duct. The rotor passage is unwrapped about a medial axis from inlet to throat as shown in Figure 6.6. As a function of streamwise distance, $\xi$, the duct area is determined from the radius of the medial circle, $h(\xi)$. A central second-order accurate finite volume discretisation is used; the equations are integrated using the SCREE scheme by Denton (2002), with dual time stepping used to recover second-order time accuracy. Sinusoidal variations of inlet relative stagnation temperature and pressure, and exit static pressure, are prescribed. The amplitude and phase of the boundary conditions are set to match time-resolved URANS computational results.

The metric for assessment of the one-dimensional model is the mean-to-peak fluctuation in static pressure coefficient on the rotor blade surface. For each location on the medial axis, this quantity is extracted from three-dimensional URANS simulations at the point when the medial circle touches the rotor pressure and suction sides, and plotted in Figure 6.7. A 'datum' case is shown in solid lines, with a supplementary 'no wake' case in dashed lines. In the latter case the upstream vane wakes are removed; the modifications necessary are described in detail in Section 6.4.1. 


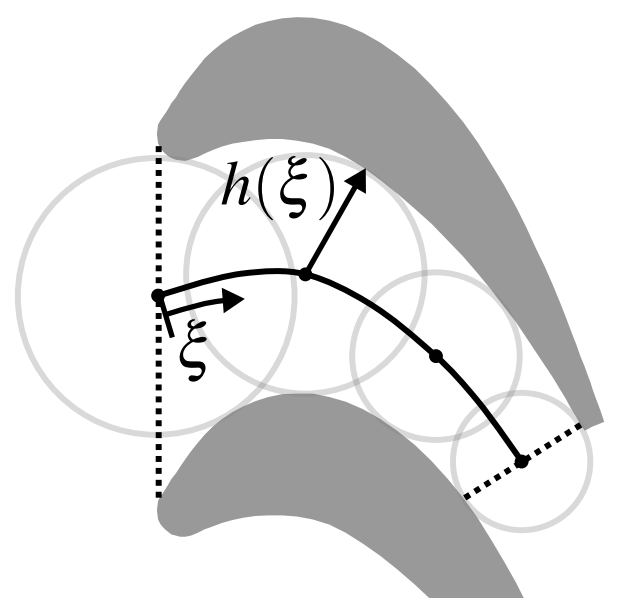

Fig. 6.6 Reduction of two-dimensional mid-span rotor passage to one-dimensional model. The passage is straightened about the medial axis into a contracting duct, with a half height equal to the medial circle radius.

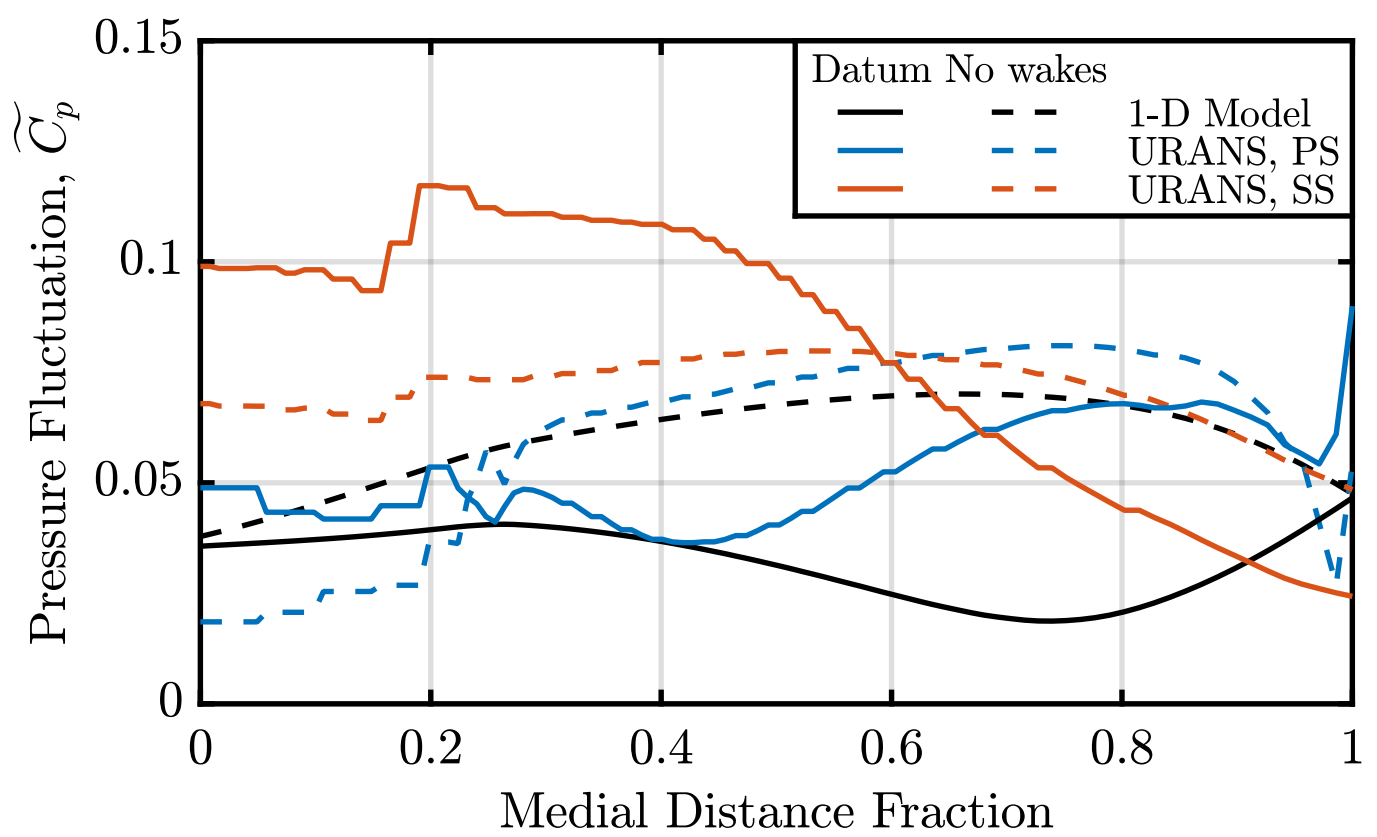

Fig. 6.7 Comparison of static pressure fluctuation amplitude on blade surface predicted by one-dimensional model and URANS computations, on pressure side (PS) and suction side (SS). The one-dimensional model agrees qualitatively if upstream wakes are removed. 
Wake interaction has a first-order effect on the amplitude of surface pressure fluctuation. The one-dimensional model is in error by up to $70 \%$ when wakes are present, but agrees qualitatively in the no wake case. This suggests that the vane potential field does propagate via approximately one-dimensional pressure waves, supporting the conclusion of Kachel and Denton (2006), although in the present turbine two-dimensional wake interaction also affects the unsteady pressure field.

\section{Unsteady streamline curvature effects}

Unsteady streamline curvature effects were first identified by Kachel and Denton (2006). The pressure gradient across a blade passage is governed by the radial equilibrium equation,

$$
\frac{\partial p}{\partial n}=\frac{\rho V^{2}}{\mathcal{R}},
$$

where $n$ is a streamline-normal coordinate, $\mathcal{R}$ is the radius of streamline curvature, and all quantities are evaluated in the rotating frame. If the streamline pattern remains approximately constant, a velocity perturbation will yield a change in pressure gradient, which when integrated to the blade surface yields a change in static pressure. This effect is two-dimensional, so is not captured by a one-dimensional duct model, and contributes to prediction errors in Figure 6.7.

To quantify the magnitude of this effect, Figure 6.8 shows the mean-to-peak variation in the right-hand side of Equation (6.4) throughout the rotor passage, calculated from the URANS computational results. A set of streamlines are traced from the rotor inlet using the time-averaged flow field to determine the local radius of curvature. Density and velocity are taken from the instantaneous flow field. The fluctuation amplitude may then be calculated, and normalised to form a pressure gradient coefficient fluctuation, $\widetilde{C_{\partial p / \partial n}}$, by the reference dynamic head and rotor pitch (ideal blade loading) according to,

$$
\widetilde{C_{\partial p / \partial n}}=\frac{\widetilde{\rho V^{2}}}{\mathcal{R}} \times \frac{\text { pitch }}{p_{\text {oref }}-p_{\text {ref }}},
$$

where all quantities are evaluated in the rotor-relative frame.

Unsteady streamline curvature effects are an order of magnitude larger on the suction

side, with up to $\widetilde{C_{\partial p / \partial n}} \approx 1$, compared to the pressure side with $\widetilde{C_{\partial p / \partial n}} \leq 0.1$. This is for two reasons. First, expanding Equation (6.4) for a perturbation in velocity $\delta V$,

$$
\delta\left(\frac{\partial p}{\partial n}\right)=\frac{2 \rho V}{\mathcal{R}} \delta V+\mathcal{O}\left(\delta V^{2}\right)
$$



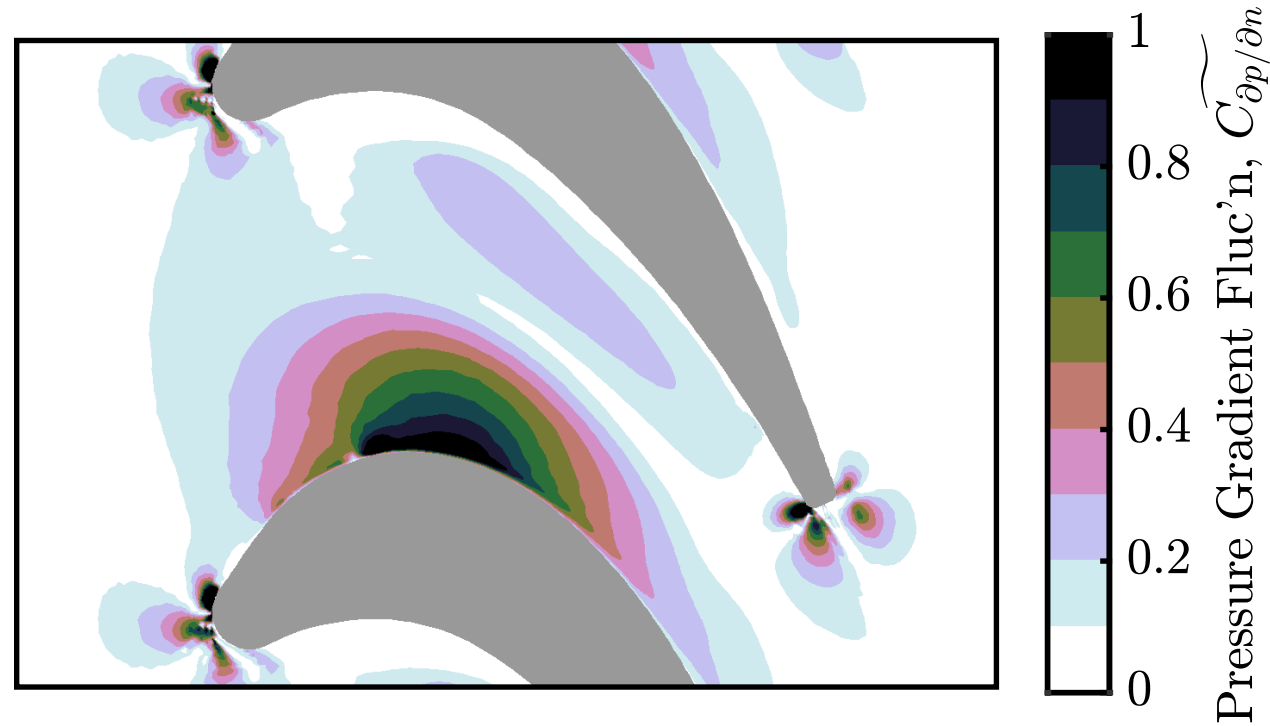

Fig. 6.8 Streamline-normal pressure gradient fluctuation amplitude at rotor mid-span. Unsteady streamline curvature effects are an order of magnitude greater on the pressure side than the suction side.

For a fixed velocity perturbation, Equation (6.6) shows that (to first order) the resulting pressure perturbation is proportional to the mean velocity, which is greater on the suction side; and inversely proportional to the radius of curvature, which is lower on the suction side. Second, the localised velocity deficit within the wake, i.e. the size of the velocity perturbation, $\delta V$, is greater on the suction side as shown in Figure 6.2.

\subsubsection{Summary of interaction mechanisms}

This section has characterised the blade row interaction mechanisms generating unsteady main-stream boundary conditions on rotor film cooling holes. The identified mechanisms are listed in Table 6.1, grouped into those generated by viscous wake non-uniformity, and the inviscid potential field.

Two mechanisms alter time-averaged stream boundary conditions: convection of wakes into the rotor with increased turbulence intensity, and redistribution of upstream vane coolant by the wake negative jet. The other mechanisms affect the instantaneous flow, causing unsteadiness in cooling hole operating point. As shown in Chapter 5, this will alter the time-averaged cooling performance if the hole is operating on a non-linear region of its characteristic. 
Table 6.1 Summary of blade row interaction mechanisms affecting rotor film cooling, divided into Potential and Wake effects. ${ }^{*}$ denotes an effect on the time-averaged flow.

\begin{tabular}{cl}
\hline \multirow{2}{*}{ Potential } & $\begin{array}{l}\text { Frozen vane pressure field } \\
\text { One-dimensional pressure waves }\end{array}$ \\
\hline \multirow{3}{*}{ Wake } & Stagnation point movement \\
& Negative-jet velocity perturbations \\
& Streamline curvature pressure perturbations \\
& Upstream vane coolant migration* \\
& Main-stream turbulence* \\
\hline
\end{tabular}

\subsection{Hole response modelling}

In this section, the modelling approach outlined in Section 6.2 is used, together with main-stream boundary conditions taken from the URANS computations analysed in Section 6.3, to predict excursions in operating point of rotor cooling holes subject to blade row interaction unsteadiness.

\subsubsection{Analysis cases}

A series of computational cases are used to separate the various blade row interaction mechanisms. The configurations of the computational model for each case are listed in Table 6.2. First, a 'Steady' case is simulated, using mixing planes to enforce steady uniform flow at the rotor inlet and exit. Simulations of this type, where blade row interaction is neglected, are typically used in the design process. The Steady case is compared to the 'Datum' case, with the same numerical approach and boundary conditions, but run time-accurate and with sliding planes. The Datum case is turbine-representative.

In addition, there are two cases in Table 6.2 where the upstream vane has been modified according to the schematics in Figure 6.9. In the 'Uncooled' case, film cooling

Table 6.2 Analysis cases for hole response modelling, with configurations of the numerical set-up in each case.

\begin{tabular}{lccc} 
& Mixing planes & Vane coolant & Vane wake \\
\hline Steady & $\checkmark$ & $\checkmark$ & $\checkmark$ \\
Datum & & $\checkmark$ & $\checkmark$ \\
Uncooled & & & $\checkmark$ \\
Potential & & & \\
\hline
\end{tabular}




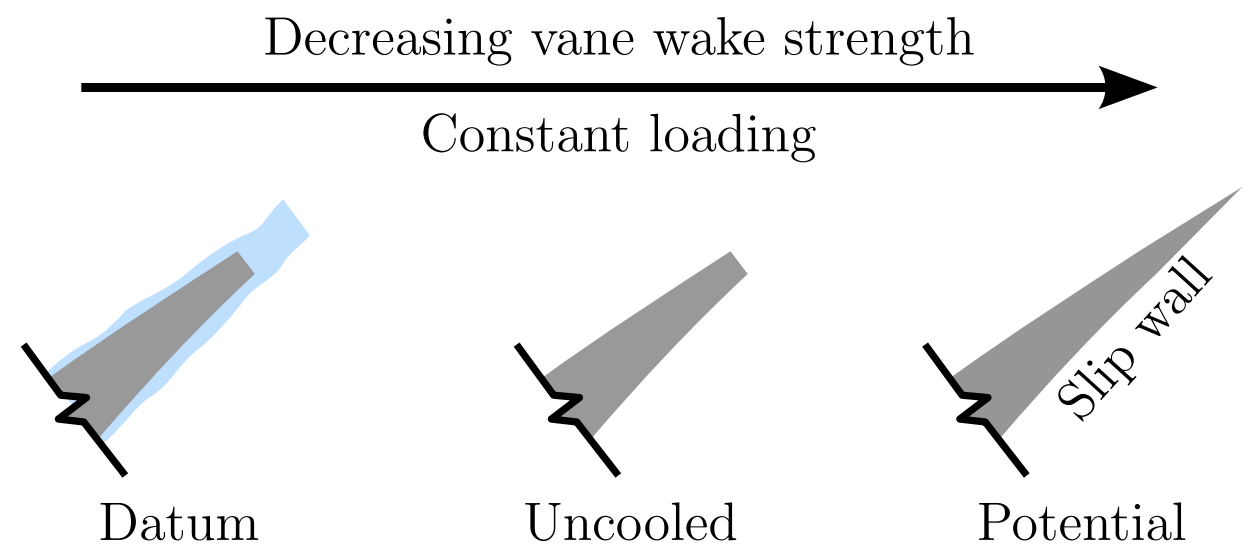

Fig. 6.9 Schematic of the modifications to upstream vane

on the upstream vane is removed, such that there is no temperature deficit within the wake. In the 'Potential' case, a sharp trailing edge and inviscid wall are used to remove base pressure loss and boundary layers respectively. Together, these eliminate the upstream vane wake (leaving only potential field non-uniformity). The modifications vary the strength of the wake negative-jet interaction. As discussed in Section 4.2, the rows are restaggered to match the vane loading and hence potential field interaction across the modified cases.

To verify the effect of modifications to the upstream vane, a comparison of the exit flow non-uniformity is shown in Figure 6.10. Mid-span pitchwise profiles of rotor-relative yaw angle and static pressure are plotted, time-averaged in the absolute frame. Figure 6.10(a) shows that removing vane coolant reduces the flow angle deficit within the wake from $25^{\circ}$ to $10^{\circ}$. When the boundary layers and base pressure losses are eliminated, in the Potential case, there is no wake, and only the inviscid flow angle non-uniformity of $\pm 6^{\circ}$ remains. The static pressure profiles in Figure 6.10(b) are similar, matching to within $\pm 1.5 \%$ of vane exit isentropic dynamic head, confirming that potential field interaction will be comparable across the three cases.

In each case, the quasi-steady model is used to predict excursions in momentum flux ratio for cooling holes placed at four locations on the rotor blade mid span. These holes are notated: 'SH', on the leading-edge showerhead; 'PS1', front pressure side; 'PS2', rear pressure side; and 'SS', suction side. The locations are shown diagrammatically in Figure 6.11. 

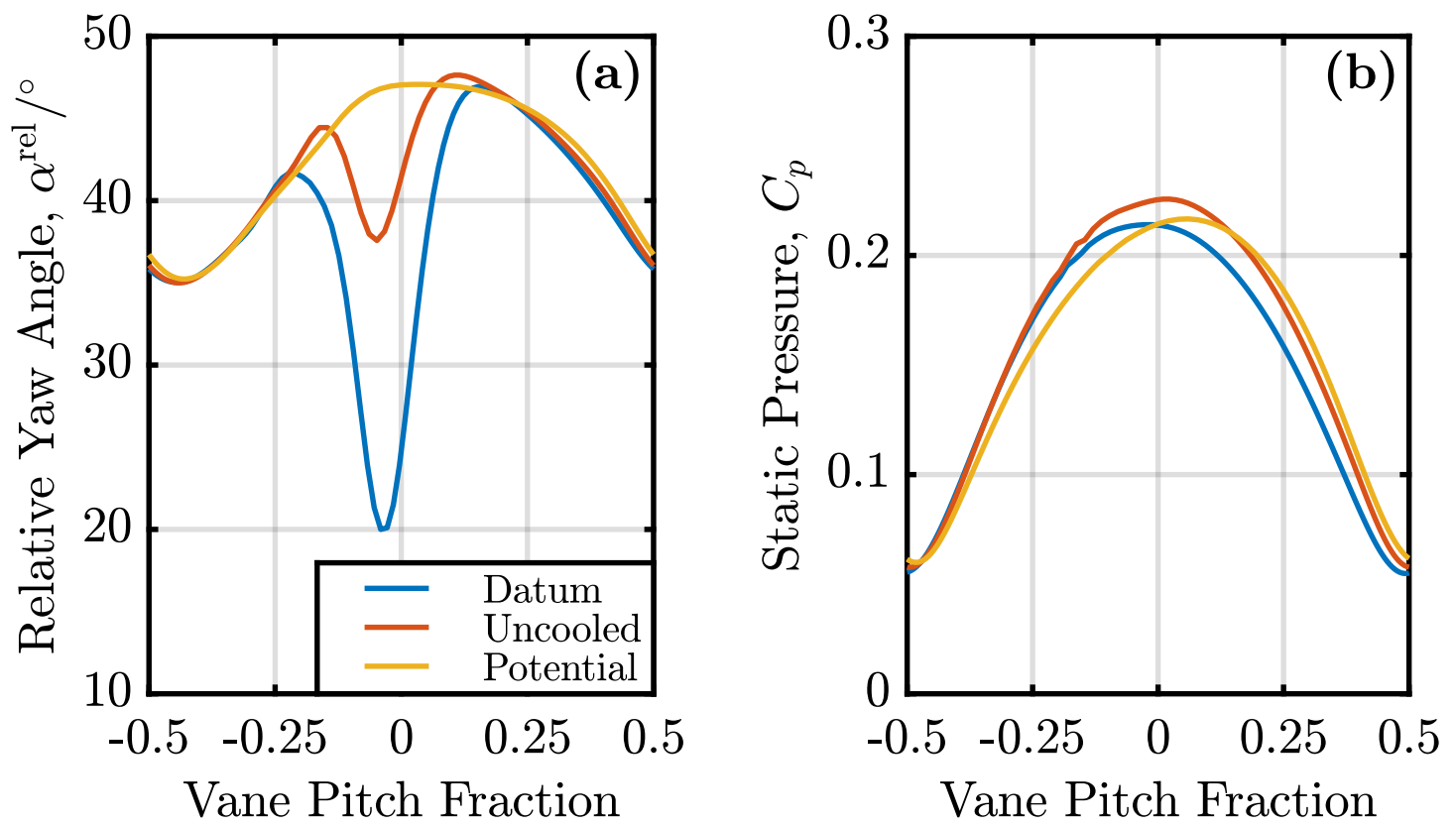

Fig. 6.10 Pitchwise profiles of upstream vane exit mid-span flow non-uniformity: (a) rotor-relative yaw angle, (b) static pressure. The modifications to the upstream vane vary the strength of the negative jet at constant potential field.

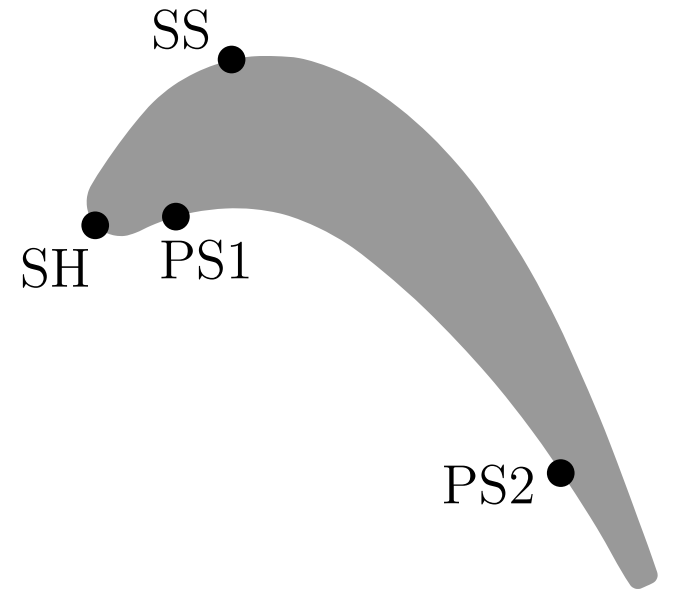

Fig. 6.11 Cooling hole locations on rotor blade mid-span. 


\subsubsection{Time-averaged cooling hole operating points}

Steady simulations are the typical computational method for turbine design iterations. The lumping of mixing loss onto mixing planes, rather than allowing a non-uniform flow to mix out naturally as it passes through the downstream row, is an approximation. Steady simulations cannot capture non-linear unsteady behaviour of either the turbine itself, or the rotor cooling holes. The present model allows quantification of error in time-averaged cooling hole operating point due to the limitations of steady computations.

The time-averaged momentum flux ratios of each cooling hole in the Steady and Datum cases are compared in Table 6.3, with the effect of unsteadiness expressed as a percentage change with respect to the Steady case. The changes are not negligible, but less than $\pm 8 \%$ for all hole locations. However, the discrepancy is due to both changes in time-averaged main-stream boundary conditions, and non-linearity in hole response, and it is of interest to separate these two effects. The former can be accounted for in steady design methods by applying main-stream boundary conditions to the cooling holes taken from a time-averaged unsteady computation, while the latter can only be dealt with through additional unsteady hole modelling as proposed in this Chapter.

In incompressible flow, the cooling hole momentum flux ratio is given by,

$$
I R=\frac{\Delta p_{\mathrm{c}} C_{\mathrm{d}}^{2}}{\frac{1}{2} \rho_{\infty} V_{\infty}^{2}}
$$

where $\Delta p_{\mathrm{c}}$ is the pressure drop across the cooling hole. Expanding Equation (6.7) for perturbations in each of the right-hand-side variables yields,

$$
\delta I R=\frac{\partial I R}{\partial \Delta p_{\mathrm{c}}} \delta \Delta p_{\mathrm{c}}+\frac{\partial I R}{\partial C_{\mathrm{d}}} \delta C_{\mathrm{d}}+\frac{\partial I R}{\partial \rho_{\infty}} \delta \rho_{\infty}+\frac{\partial I R}{\partial V_{\infty}} \delta V_{\infty}+\mathcal{O}\left(\delta^{2}\right)
$$

Table 6.3 Time-averaged momentum flux ratios of each rotor cooling hole, predicted by quasi-steady model, for Datum and Steady cases.

\begin{tabular}{clllll} 
& Hole location: & SH & PS1 & PS2 & SS \\
\hline \multirow{2}{*}{ Time-averaged } & Steady case & 2.20 & 1.72 & 0.50 & 0.51 \\
Momentum Flux Ratio, IR & Datum case & 2.03 & 1.75 & 0.49 & 0.53 \\
& Change, \% & -7.8 & 1.3 & -0.8 & 3.7 \\
\hline
\end{tabular}


By differentiating Equation (6.7), normalised linear influence coefficients for each righthand-side variable may be derived,

$$
\begin{aligned}
& \frac{\partial I R}{\partial \Delta p_{\mathrm{c}}}\left(\frac{\Delta p_{\mathrm{c}}}{I R}\right)=1, \\
& \frac{\partial I R}{\partial C_{\mathrm{d}}}\left(\frac{C_{\mathrm{d}}}{I R}\right)=2, \\
& \frac{\partial I R}{\partial \rho_{\infty}}\left(\frac{\rho_{\infty}}{I R}\right)=-1, \\
& \frac{\partial I R}{\partial V_{\infty}}\left(\frac{V_{\infty}}{I R}\right)=-2 .
\end{aligned}
$$

Combining Equations (6.9) and (6.8) gives the following compact description of momentum flux ratio perturbations,

$$
\hat{\delta} I R=\hat{\delta} \Delta p_{\mathrm{c}}+2 \hat{\delta} C_{\mathrm{d}}-\hat{\delta} \rho_{\infty}-2 \hat{\delta} V_{\infty}+\mathcal{O}\left(\hat{\delta}^{2}\right)
$$

where $\hat{\delta}$ denotes a fractional perturbation, such as $\hat{\delta} I R=\delta I R / I R$. The sum of first-order terms on the right-hand side of Equation (6.10) is by construction the linear change in momentum flux ratio, and the second-order terms correspond to non-linear effects. Grouping all non-linear effects into a single term, $\Sigma$, Equation (6.10) reads,

$$
\hat{\delta} I R=\hat{\delta} \Delta p_{\mathrm{c}}+2 \hat{\delta} C_{\mathrm{d}}-\hat{\delta} \rho_{\infty}-2 \hat{\delta} V_{\infty}+\Sigma
$$

For a sufficiently small change in steady boundary conditions, in the absence of unsteady fluctuations, the momentum flux ratio perturbation will be approximately linear. Comparing the Steady case, and a time-invariant case with the time-averaged Datum boundary conditions, the non-linear term is small at $\Sigma \leq 0.5 \%$. This confirms the applicability of Equation (6.11).

Figure 6.12 shows the contributions of each term in Equation (6.11) to the effect of blade row interaction on the time-averaged momentum flux ratios of each rotor cooling hole. As in Table 6.3, the perturbations are defined relative to the Steady case. The effect of unsteadiness on the time-averaged discharge coefficient is negligible. The time-averaged pressure drop across all cooling holes is reduced by unsteadiness, by between $1.3 \%$ and $3.0 \%$, despite the fact that the coolant pressure margin is held constant at $3 \%$ over the time- and mass-averaged rotor inlet relative stagnation pressure in both cases. The reason for the consistent, small reduction in static pressure at the cooling hole exits is not clear. 


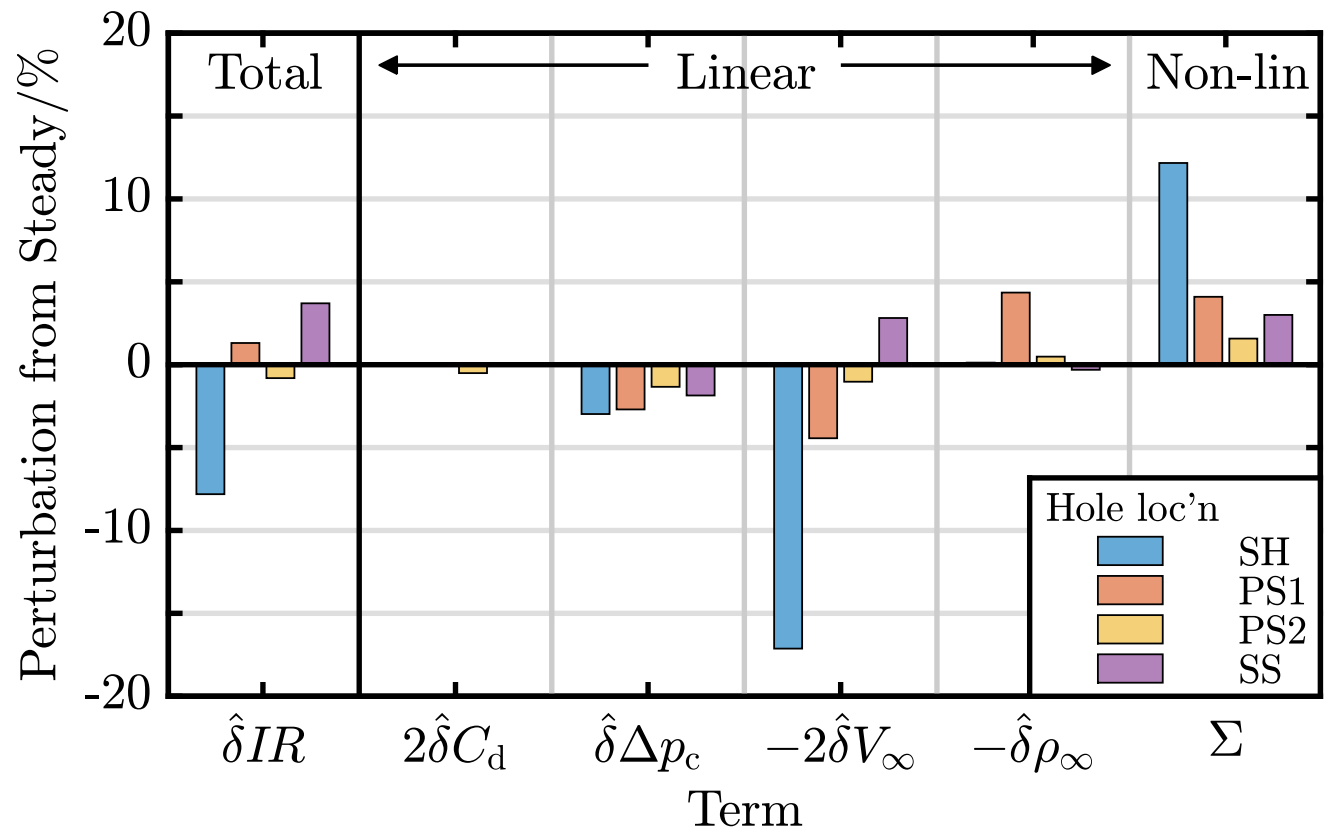

Fig. 6.12 Breakdown of linear and non-linear contributions to the effect of blade row interaction on time-averaged momentum flux ratio. Each of the terms in Equation (6.11) is plotted separately for four rotor cooling hole locations. $\hat{\delta}$ is a fractional perturbation in boundary condition or flow property, and $\Sigma$ includes non-linear effects.

The largest contributions to change in momentum flux ratio come from the change in time-averaged main-stream velocity: $+16 \%$ at the showerhead hole and $+4 \%$ at the front pressure-side hole. These holes are situated near the rotor leading edge, where the wake is distorted as it impacts the blade and causes large velocity perturbations, and the time-averaged effect is an increase in velocity. Change in main-stream density plays a small role at all holes excluding the front pressure-side hole, where a decrease of $4 \%$ is apparent. This is because high-density coolant is mixed-out before entering the rotor in the Steady simulation, and thus distributed evenly across the blade passage. Conversely, in the unsteady computation, the negative jet causes coolant to migrate towards the suction side, leaving a relatively lower density on the pressure side.

The balance of the momentum flux ratio change, after the linear terms have been accounted for, is non-linear effects, $\Sigma$. Non-linear effects act to increase the time-averaged momentum flux ratio at all hole locations, from 1.6\% at hole PS2 up to $12 \%$ at hole SH. The effect is largest at the showerhead hole because it experiences the largest main-stream velocity excursions during wake impact. Differentiating Equation (6.7) twice with respect 
to $I R$, the normalised second-order influence coefficient for main-stream velocity is,

$$
\frac{\partial^{2} I R}{\partial V_{\infty}^{2}}\left(\frac{V_{\infty}^{2}}{I R}\right)=5 .
$$

Because $\partial^{2} I R / \partial V_{\infty}^{2}$ is positive, the instantaneous momentum flux ratio is higher than that given by a linear hole. So, for sufficiently large velocity fluctuations, non-linear effects will act to increase the time-averaged momentum flux ratio, consistent with the observed positive values of $\Sigma$

The implication of Figure 6.12 is that the effect of blade row interaction on timeaveraged momentum flux ratio cannot be accounted for solely by using main-stream boundary conditions taken from a time-averaged unsteady computation. This would result in an error of $12 \%$ for the showerhead hole, for example.

\subsubsection{Instantaneous cooling hole operating points}

Quasi-steady model predictions of instantaneous momentum flux ratio for each the four cooling hole locations are shown in Figure 6.13. Traces are shown for all four cases in Table 6.2. Beginning with the Datum case, each hole experiences excursions in momentum flux ratio of not less than $\pm 30 \%$. For hole $\mathrm{SH}$ on the leading-edge showerhead, the maximum momentum flux ratio is $240 \%$ of the time-averaged value. The cooling hole characteristic cannot in general be assumed linear over such a large range, suggesting that non-linear effects need to be considered.

Now considering the modified vane cases, if potential field interaction was the only driver of rotor film cooling unsteadiness, the traces for each modified vane would be identical, because the vane loading is held constant. This is approximately the case for some portions of the vane passing cycle. For example, $0.4 \leq f t \leq 0.8$ in Figure 6.13(a) and $0.2 \leq f t \leq 0.5$ in Figure 6.13(c). These times correspond to instants where the vane wake is remote from the cooling hole, and so does not perturb the operating point, leaving only potential field interaction which is common to all cases. However, when the vane wake is over the cooling hole, it alters the main-stream velocity and hence momentum flux ratio. On the pressure side, Figure 6.13(c), the negative jet reduces main-stream velocity as it approaches the hole at $f t=0.8$, increasing momentum flux ratio to $40 \%$ over the time averaged value. After the wake passes at $f t=1.0$, the main-stream velocity increases, and hence momentum flux ratio reduces to $24 \%$ below the time average. Consistent with established negative jet kinematics, a converse process takes place on the suction side, Figure 6.13(d). 


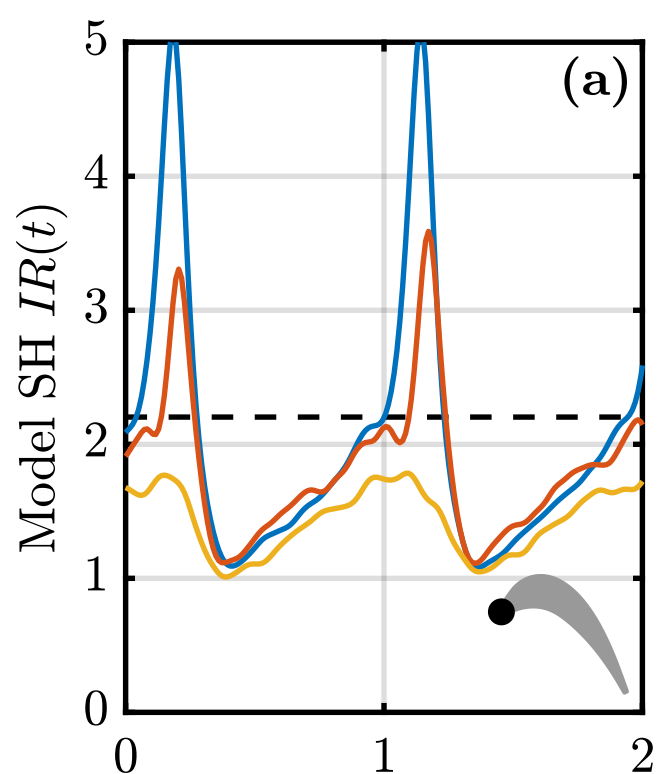

Vane Passing Periods, ft

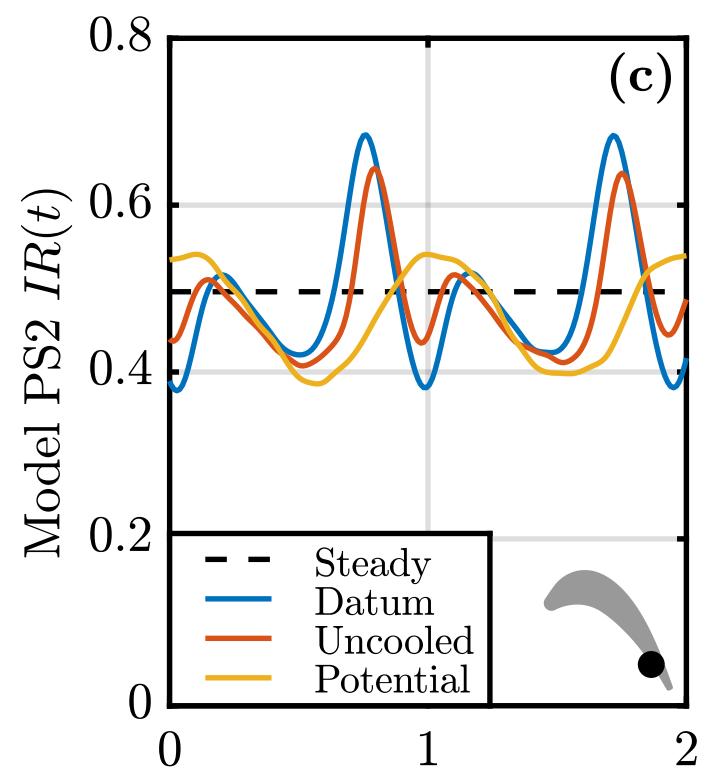

Vane Passing Periods, $f t$

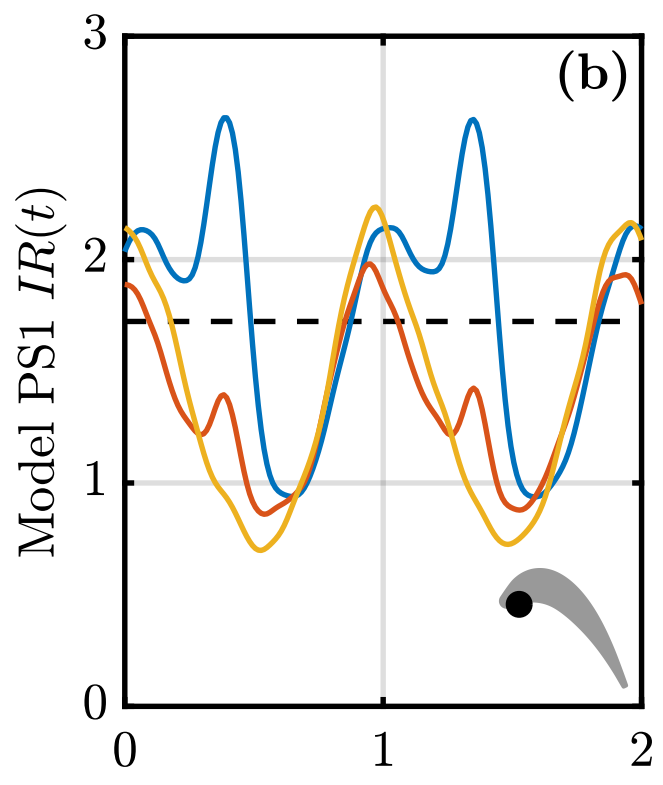

Vane Passing Periods, ft

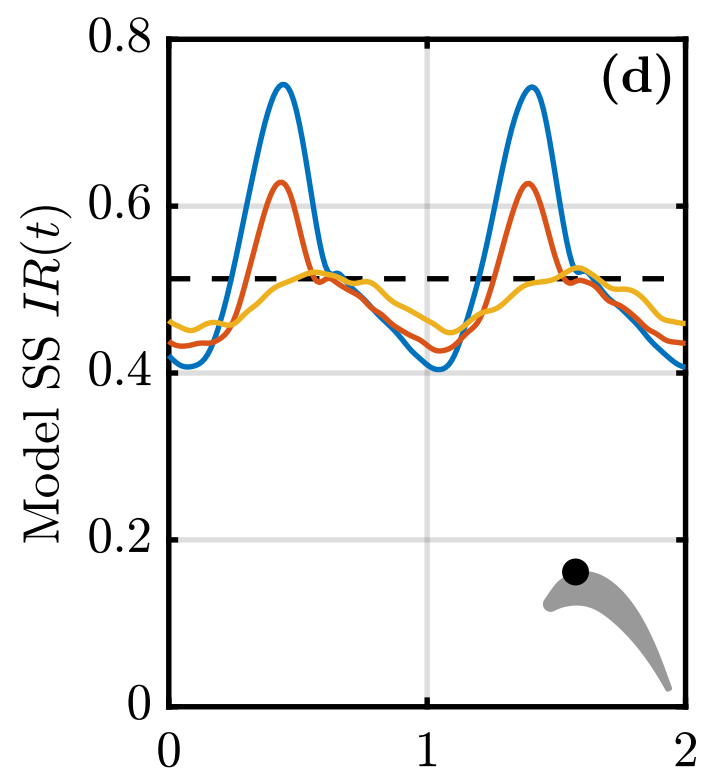

Vane Passing Periods, ft

Fig. 6.13 Instantaneous momentum flux ratio of rotor mid-span cooling holes, predicted by quasi-steady model, for hole locations: (a) showerhead, $\mathrm{SH}$; (b) front pressure side, PS1; (c) rear pressure side, PS2; (d) suction side, SS. Excursions in momentum flux ratio of at least $\pm 30 \%$ are observed in the Datum case, and removing wake interaction reduces the level of unsteadiness. 
The perturbation associated with the wake is larger than that associated with the potential field, so that not modelling wake interaction reduces momentum flux ratio excursions in the Potential case. For example, for the suction side hole in Figure 6.13(d), the magnitude of excursions reduces from $\pm 32 \%$ to $\pm 8 \%$. On the pressure side, where negative-jet velocity perturbations are smaller, the amplitude of the wake effect is greater than the potential field by a factor of approximately 2. On the suction side, larger velocity perturbations cause the wake effect to be greater by up to a factor of 5 .

The instantaneous momentum flux ratio in the Uncooled case is generally bracketed by the Datum and Potential cases. This is because the strength of the wake negative jet is augmented by vane coolant in the Datum case, and removed in the Potential case, with the Uncooled an intermediate situation. This is most clearly illustrated on the suction side, Figure 6.13(d), where the departure of the Datum trace with respect to the Potential is approximately twice that of the Uncooled trace.

The timing of the wake with respect to the potential field also affects the instantaneous momentum flux ratio. For the front pressure side hole in Figure 6.13(b), the wake arrives during a trough in the Potential momentum flux ratio, $f t=0.4$, so the maximum momentum flux is only $155 \%$ of the time-averaged value. For the showerhead hole in Figure 6.13(a), wake interaction occurs during a peak at $f t=0.2$, and the momentum flux ratio rises to $240 \%$ of the time-averaged value.

While wake interaction effects tend to produce larger momentum flux ratio perturbations than the potential field, both play a role in setting the instantaneous momentum flux ratio. It can be shown (Greitzer et al., 2004) that the amplitude of potential field distortions decays exponentially with distance according to,

$$
p^{\prime} \propto \exp \left(\frac{-2 \pi x}{S} \sqrt{1-M a^{2}}\right)
$$

where $S$ is the circumferential pitch. This implies that the balance between wake and potential field interactions is a function of row spacing and vane exit Mach number. In particular, the amplitude of potential field interactions will increase in highly-loaded turbines.

\subsection{Recommendations for design}

In this section, results of the URANS computations and quasi-steady modelling are used to make recommendations for the design of film cooling accounting for blade row interaction effects. 
Target a linear hole response The modelling in Section 6.4.3 confirms that first-order momentum flux ratio excursions will occur due to main-stream unsteadiness generated by blade row interaction in a representative turbine. As shown in Chapter 5, if the hole characteristic is non-linear, unsteadiness in operating point will alter the time-averaged cooling performance. Therefore, to avoid non-linear unsteady effects, the designer should choose a hole characteristic, and time-averaged position on that characteristic, such that the hole behaves linearly over the expected range of unsteady excursions. The extent of unsteady movement on the characteristic can be found using a quasi-steady model as described here.

Include vane coolant redistribution With a uniform upstream stagnation temperature, the adiabatic wall temperature at turbine conditions can be found by scaling film effectiveness measurements from laboratory flat-plate and cascade tests. However, a cooled upstream vane results in a non-uniform upstream stagnation temperature, which is then redistributed by the negative jet throughout the blade passage. This implies that scaling film effectiveness data will not result in the correct adiabatic wall temperature, because the local main-stream temperature is non-uniform and not known a-priori. To produce a credible design prediction of rotor cooling performance, redistribution of vane coolant due to wake interaction must therefore be included in the analysis.

Avoid time-averaged stagnation point The stagnation point is found to oscillate by $\pm 12^{\circ}$ on the rotor leading edge due to wake interaction. If the stagnation point crosses a cooling hole, film effectiveness is reduced via non-linear effects, so the designer should position showerhead holes outside of the expected stagnation point motion range. More realistically, if a designer were to place two cooling holes at, say, $\pm 10^{\circ}$ about the time-averaged stagnation point, the predicted range of motion will still produce first-order excursions in the showerhead cooling hole momentum flux ratio, and a deficit in performance compared to steady-state estimates is expected due to hole response non-linearity.

Reduce negative jet strength Results from the modified vane cases show that momentum flux ratio perturbations associated with wake interaction are larger than those from the potential field. This suggests that reducing the strength of the upstream vane negative jet will mitigate unsteady effects on rotor cooling. The turbine designer has little control over the magnitude of the velocity deficit, but the choice of velocity triangle alters the corresponding effect in the relative frame. It can be shown that, for a 


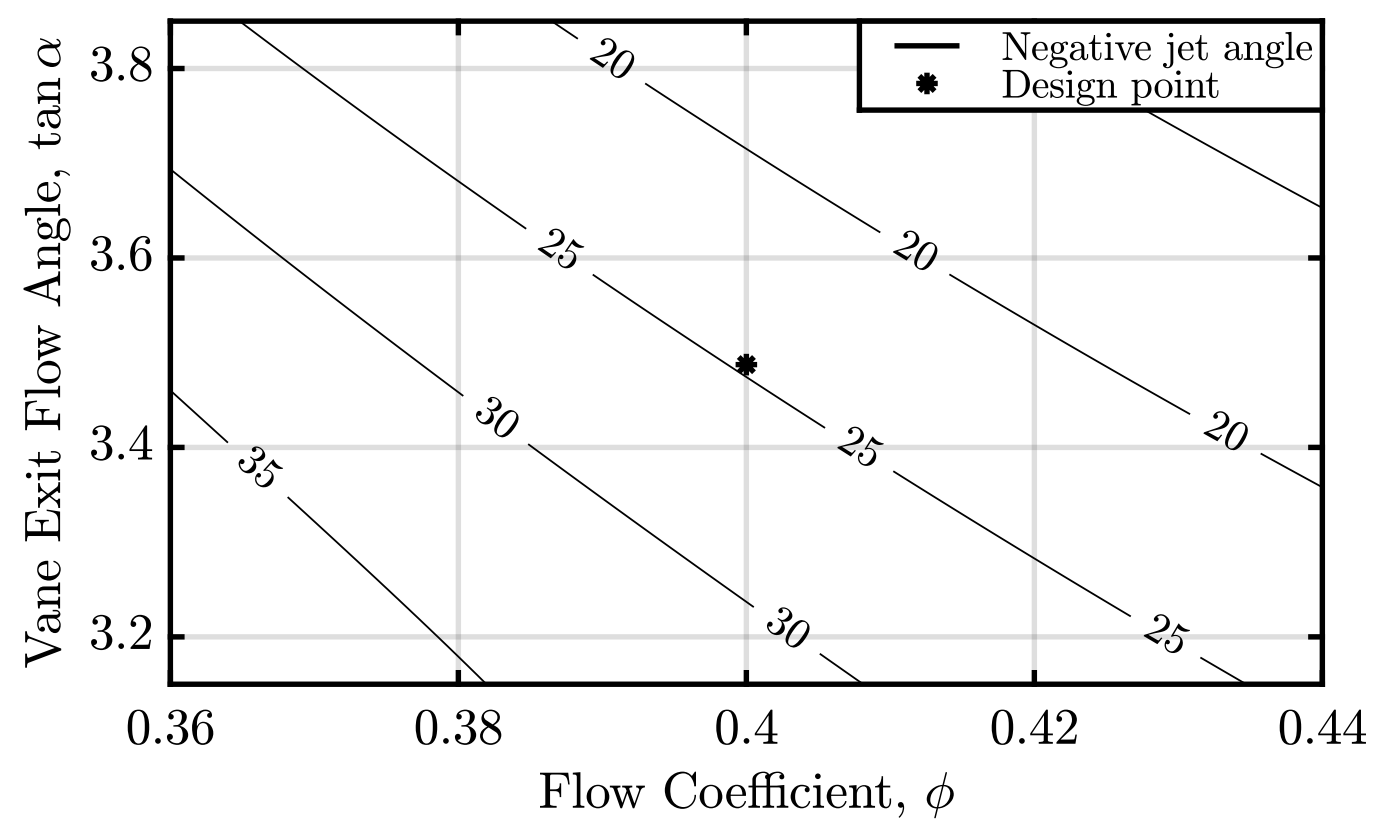

Fig. 6.14 Effect of velocity triangle design on negative jet strength. Contours of wake relative flow angle deficit for variations of $\pm 10 \%$ in flow coefficient and vane exit flow angle tangent about design point. Increased loading, by either increased flow coefficient or increased vane turning, diminishes the negative jet.

flow coefficient, $\phi$, and absolute yaw angle, $\alpha$, the relative frame yaw angle $\alpha^{\text {rel }}$ is given by,

$$
\tan \alpha^{\mathrm{rel}}=\tan \alpha-1 / \phi
$$

By applying Equation (6.14) separately to wake and free-stream fluid, which have differing flow coefficient but the same absolute yaw angle, the relative yaw angle deficit within the wake can be calculated. This is the 'negative jet angle', taken as a measure of the strength of wake interaction. Figure 6.14 shows this quantity plotted for variations of $\pm 10 \%$ about the design point flow coefficient and absolute flow angle tangent, with a constant wake velocity deficit of $20 \%$. The negative jet strength can be reduced by either increasing the flow coefficient, or increasing the vane turning, both of which act to increase loading. 


\subsection{Conclusions}

In this Chapter, the mechanisms of blade row interaction that affect rotor film cooling are identified and illustrated using non-film resolved computations of a turbine stage, representative of a large industrial gas turbine. A quasi-steady hole response model is formulated and used to predict unsteady excursions in momentum flux ratio of rotor cooling holes. The following conclusions are drawn from the presented results:

1. The upstream vane wake profile produces a negative-jet perturbation on interaction with the rotor. Instantaneously, this perturbs the velocity field by up to $\pm 15 \%$ isentropic exit velocity. The time-averaged effect is migration of vane coolant from pressure side to suction side, causing main-stream temperature variations equivalent to \pm 0.025 in blade film effectiveness.

2. The rotor leading-edge stagnation point oscillates in response to a non-uniform upstream flow angle, by $\pm 12^{\circ}$. The rotor inlet flow angle distortion is generated by the velocity and temperature deficit of the cooled vane wake.

3. Vane potential field interaction creates pressure perturbations, moving circumferentially in the rotor-relative frame, upstream and downstream of the rotor. The downstream vane potential field, with a smaller pitch in this case, decays to a negligible level before reaching the rotor, unlike the upstream vane potential field, with a larger pitch. Pressure fluctuations are present throughout the rotor passage, of up to $\pm 10 \%$ isentropic exit dynamic head.

4. Modelling the propagation of pressure perturbations as a one-dimensional waves yields qualitative predictions of static pressure fluctuations on the blade surface only for a case without upstream vane wakes. This suggests that while the potential field does propagate as approximately one-dimensional waves, two-dimensional wake interaction also contributes to static pressure unsteadiness.

5. The reduced frequency of a rotor cooling hole is low, $\kappa_{\mathrm{c}} \approx 0.06$, so that a quasisteady model can be used to predict excursions in hole operating point, using main-stream boundary conditions from unsteady non-film-resolved computations. The momentum flux ratio of all cooling holes is observed to fluctuate by at least $\pm 30 \%$ due to main-stream unsteadiness (points 1 and 3). As shown in Chapter 5, if the hole is operating on a non-linear characteristic, this will affect the time-averaged cooling performance. 
6. A series of modified upstream vanes are used to vary the strength of wake interaction at constant potential field, in order to isolate their separate effects. In general, both mechanisms contribute to hole operating point unsteadiness. For cooling holes on the pressure surface, the effect of wake interaction is greater than the potential field by a factor of two, while on the suction surface and leading edge, the wake effect is greater by up to a factor of five.

7. Based on these observations, the following recommendations are made for the design of rotor film cooling in the real, unsteady turbine environment:

(a) The designer should choose a cooling hole characteristic and nominal timeaveraged operating point such that the hole responds linearly over the expected unsteady excursions, found using a quasi-steady model (point 5).

(b) Redistribution of vane coolant throughout the rotor passage due to wake interaction cannot be neglected in a credible design prediction of cooling performance (point 1).

(c) Leading edge showerhead cooling holes should be placed outside of the unsteady motion range of the stagnation point, which may be determined from the upstream flow angle non-uniformity (point 2). 



\title{
Chapter 7
}

\section{Film-resolved flat plate simulations}

\begin{abstract}
In order to validate the hybrid URANS-LES computational approach described in Chapter 4, simulations are presented of cylindrical and laid-back fan-shaped film cooling holes on a flat plate, each at low and high blowing ratios, for which high-quality experimental measurements are available.

The flow features predicted by the computations are in accordance with the existing understanding of cylindrical and shaped hole film cooling. Good agreement with laterally-averaged film effectiveness data is obtained in all cases, tending towards over-prediction by between $+11 \%$ and $+23 \%$. The accuracy of these predictions is state-of-the-art.

The hybrid computational approach is zonal, in that the boundary between the URANS and LES zones is selected by the user. It is found that film effectiveness results are insensitive to the choice of URANS layer thickness for values greater than $6 \%$ hole diameter. Furthermore, a consistent, fixed, computational model is shown to be successful in two different geometries at both low and high blowing ratios. These results give confidence in the general predictive capability of the present approach.
\end{abstract}




\subsection{Introduction}

In this Chapter, a hybrid URANS-LES computational method is validated across four film cooling test cases. Full details of the numerical method including the meshing, geometry and computation procedures are given in Section 4.3. The test cases are single rows of holes on a flat plate, with two different hole geometries, each at low and high blowing ratios.

In the open literature, the number of experimental studies suitable for computational model validation is limited. The transonic test rig at the University of Karlsruhe, first described by Wittig et al. (1996), uses known temperature distributions applied to a solid conduction model of the cooled surface to yield high-accuracy film effectiveness measurements, accurate to within $\pm 4 \%$, the first requirement for computational model validation. The present simulations target the hole geometries reported by Saumweber et al. (2003), where the coolant and main-stream boundary conditions are documented in sufficient detail, the second requirement for computational model validation. The boundary-layer thickness is the only parameter which is not unambiguously defined, as discussed further in Section 7.2.

Table 7.1 summarises the experimental non-dimensional parameters for the two geometries: cylindrical and laid-back fan-shaped holes. The hole shapes are shown in detail in Figure 7.1. Excluding the main-stream turbulence intensity of $T u=3.6 \%$, which is neglected, all other parameters are matched in the present computations. There are no suitable reference film effectiveness measurements in the open literature with negligible main-stream turbulence, $T u \leq 1 \%$, that provide complete documentation of boundary conditions. The commonly-used case of Sinha et al. (1991) does not include measurements of the upstream boundary-layer thickness. It is preferable to target the Saumweber et al. (2003) study, because they quantify the effect of varying $T u$, whereas it is not possible to estimate the uncertainty associated with boundary-layer thickness from the Sinha et al. (1991) data.

The Chapter is organised as follows. First, a simulation with no cooling hole is presented to compare with the experimental boundary layer measurements. Then, the cylindrical hole cases are discussed, followed by the shaped hole cases. For each geometry, results are presented to illustrate the resolution of the coolant film, and the predicted distributions of film effectiveness downstream of the cooling hole are compared with measured data. The purpose is to demonstrate the predictive capability of the present computational approach. 
Table 7.1 Non-dimensional geometry and boundary condition parameters for flat plate film cooling validation test cases, from Saumweber et al. (2003).

\begin{tabular}{ll}
\hline Hole lateral expansion angle, $\chi_{z}$ & $0^{\circ}, 14^{\circ}$ \\
Hole wall-normal expansion angle, $\chi_{y}$ & $0^{\circ}, 15^{\circ}$ \\
Hole inclination angle, $\alpha$ & $30^{\circ}$ \\
Hole pitch-to-diameter ratio, $P / D$ & 4 \\
Hole length-to-diameter ratio, $L / D$ & 6 \\
Main-stream Mach number, $M a_{\infty}$ & 0.3 \\
Main-stream Reynolds number, $R e_{\infty}$ & 25000 \\
Main-stream turbulence intensity, $T u_{\infty}$ & $3.6 \%$ \\
Coolant temperature ratio, $T_{0 \mathrm{c}} / T_{0 \infty}$ & 0.57 \\
Coolant blowing ratio, $B R$ & $0.5,1.5,2.5$ \\
\hline
\end{tabular}
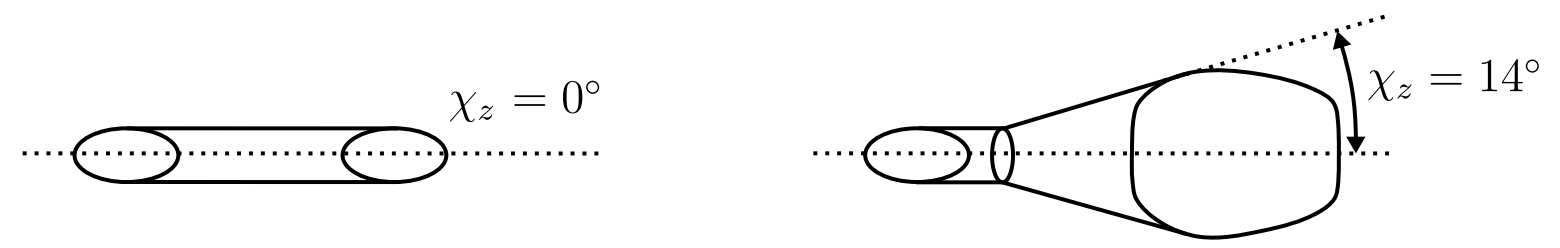

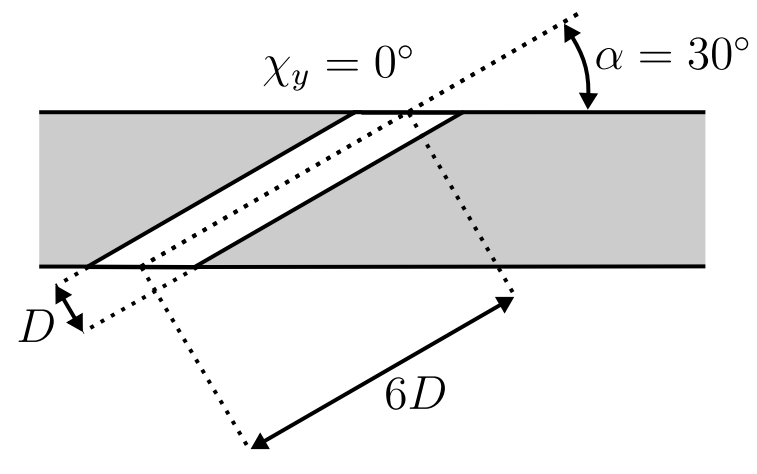

(a) Cylindrical

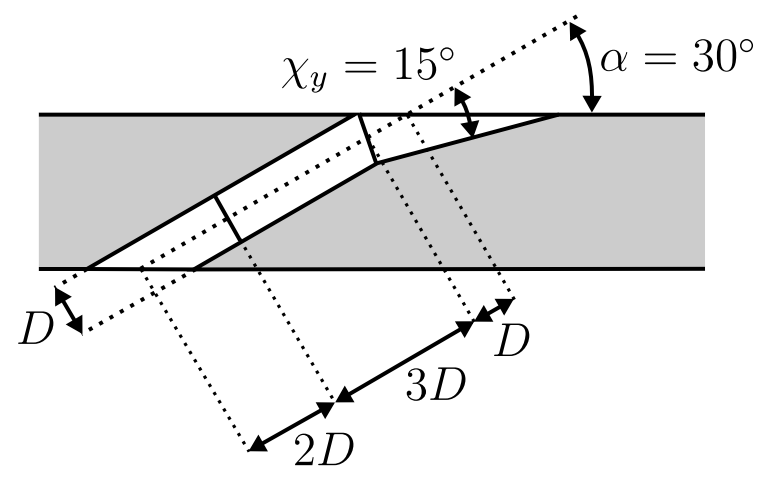

(b) Laid-back fan-shaped

Fig. 7.1 Hole geometries for film-resolved computations, after Saumweber et al. (2003). 


\subsection{Boundary layer}

Film effectiveness is affected by boundary-layer state and thickness, so any prediction of cooling performance must include the correct upstream boundary layer. Simulations of a flat plate with no cooling hole are used to verify that the boundary layer in the present computational model is representative of the target experimental conditions. The mesh has the same density in the streamwise, wall-normal, and lateral directions as the cooling hole cases described later: $\Delta x^{+} \approx 50, \Delta y^{+} \approx 1$, and $\Delta z^{+} \approx 25$ respectively. The boundary conditions are also the same as the cooling hole cases, omitting the coolant inlet.

Flow on the inlet boundary situated at $x / D=-15$ is uniform at the main-stream stagnation pressure and temperature. A boundary-layer trip is located at $x / D=-14$, a rectangular region of reversed streamwise velocity $V_{x} / V_{\infty}=-0.7$ covering the full lateral extent of the domain. The trip height is $0.025 D$, one fifth of the target boundary-layer displacement thickness, and the trip measures $0.1 D$ in length. The inflectional velocity profile caused by the trip is unstable and breaks down into a turbulent boundary layer.

The transition process is quantified using the boundary-layer shape factor, the ratio of displacement to momentum thicknesses $\delta_{*} / \theta$. In turn, these are defined,

$$
\begin{aligned}
\delta_{*} & =\frac{1}{P} \int_{-P / 2}^{P / 2} \int_{0}^{y_{\infty}}\left(1-\frac{\rho V_{x}}{\rho_{\infty} V_{\infty}}\right) \mathrm{d} y \mathrm{~d} z, \\
\theta & =\frac{1}{P} \int_{-P / 2}^{P / 2} \int_{0}^{y_{\infty}} \frac{\rho V_{x}}{\rho_{\infty} V_{\infty}}\left(1-\frac{\rho V_{x}}{\rho_{\infty} V_{\infty}}\right) \mathrm{d} y \mathrm{~d} z,
\end{aligned}
$$

where subscript $\infty$ denotes the main-stream location taken at $y_{\infty} / D=4$. The shape factor is calculated for a series of planes at different streamwise locations and plotted in Figure 7.2. After the trip at $x / D=-14$, the shape factor approaches the characteristic fully-turbulent value of 1.4 , reaching 1.36 by $x / D=-10$, and remaining constant at $\delta_{*} / \theta=1.42 \pm 0.8 \%$ for $x / D \geq-5$. The small variation in shape factor for $x / D \geq-5$ indicates that the boundary layer is self-similar and in an equilibrium state.

Boundary-layer velocity profiles just upstream of the cooling hole, at $x / D=-1.3$, are plotted in Figure 7.3. In the outer layer, the velocity profile is compared with the empirical $1 / 7$ th power fit,

$$
\frac{V_{x}}{V_{\infty}}=\left(\frac{y}{\delta_{99 \%}}\right)^{1 / 7}
$$




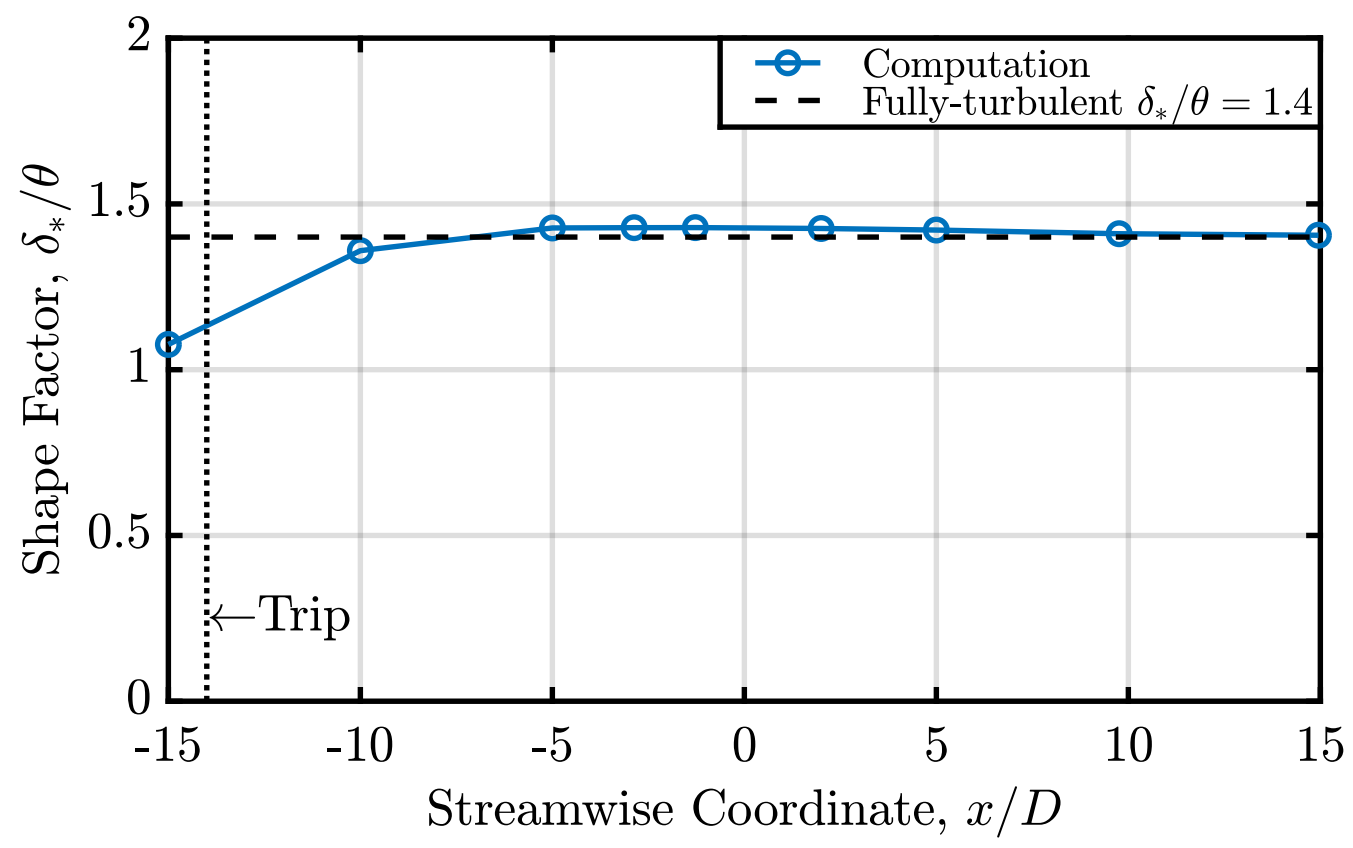

Fig. 7.2 Streamwise development of flat plate boundary-layer shape factor. After being tripped at $x / D=-14$, the profile is in a fully-turbulent, self-similar state for $x / D \geq-5$, with a shape factor equal to $\delta_{*} / \theta=1.42 \pm 0.8 \%$.

where $\delta_{99 \%}$ is the $99 \%$ velocity thickness, i.e. the wall-normal location at which the streamwise velocity reaches $99 \%$ of the main-stream value. Figure 7.3 (a) shows that the predicted velocity is in agreement with the fit to within $\pm 2.3 \% V_{\infty}$ for $y / \delta_{99 \%} \geq 0.2$.

In the inner layer, $y / \delta_{99 \%} \leq 0.2$, the velocity profile is shown in wall units in Figure 7.3(b) and compared to the universal log law for a turbulent boundary layer, and a linear profile in the laminar sublayer. A limitation of the mixing-length wall model is apparent - the laminar sublayer is not captured, and instead the velocity profile is logarithmic throughout the URANS layer, from $y^{+}=50$ all the way to the wall. This is because there is no mechanism in the mixing-length model to identify the grid points located in the laminar sublayer (seven for $y^{+} \leq 10$ ) and reduce turbulent viscosity accordingly. A numerical 'buffer layer' is present for $100 \leq y^{+} \leq 150$ as the turbulent shear stress blends from modelled in the URANS layer to resolved in the LES region. For $y^{+} \geq 150$, a logarithmic layer is resolved, with an effective von Kármán constant of $\kappa \approx 0.5$ and offset by six friction velocities below the universal log law due to errors closer to the wall.

Despite modelling deficiencies in the inner region of the boundary layer, it is concluded from the results of Figures 7.2 and 7.3 that the boundary layer is modelled with sufficient 

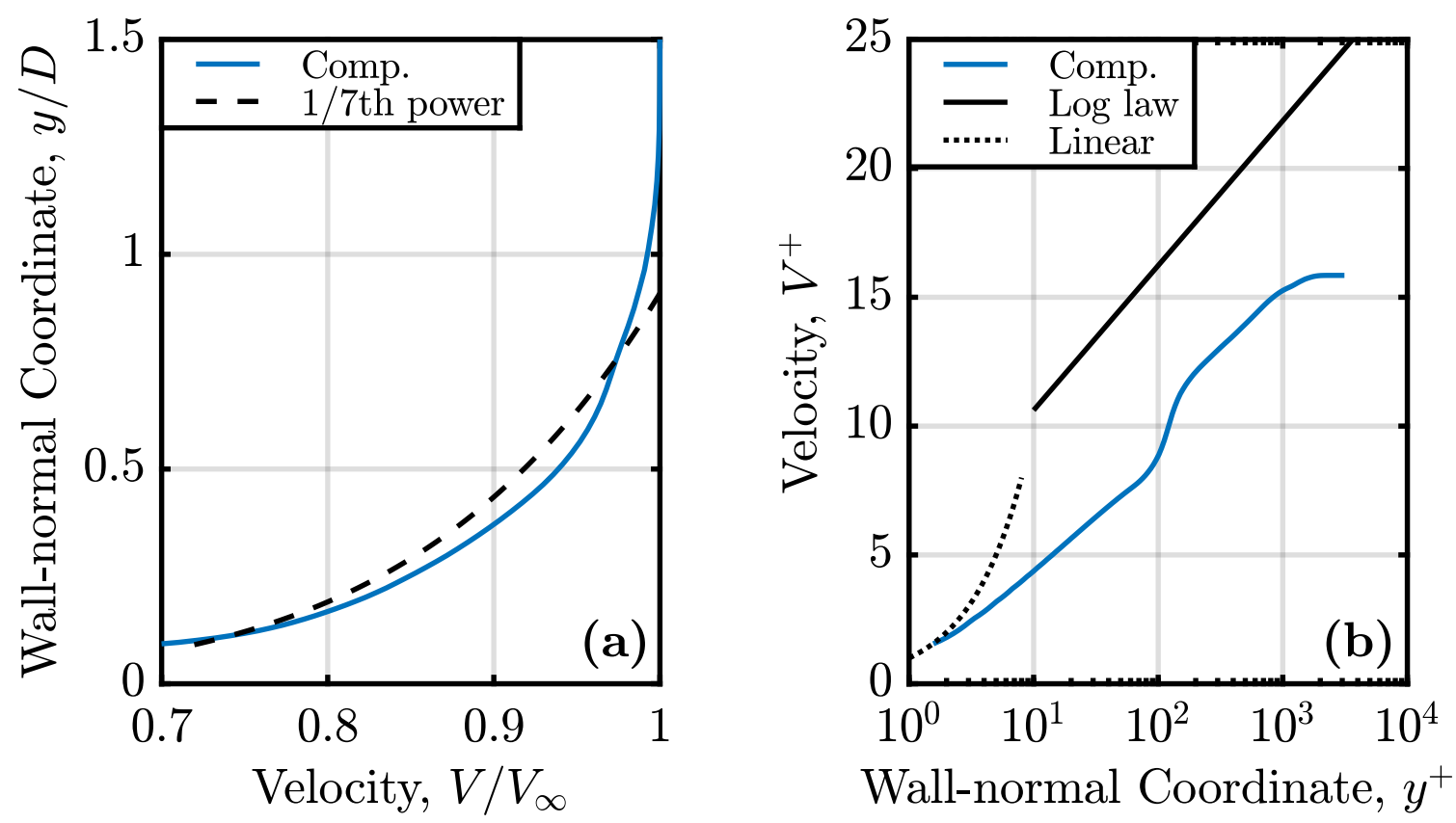

Fig. 7.3 Comparison of flat plate boundary-layer velocity profiles at $x / D=-1.3$ against analytic results: (a) outer units, a 1/7th power curve fits the outer profile to within $\pm 2.3 \% V_{\infty}$ for $0.2 \geq y / \delta_{99 \%} \geq 0.95$; (b) wall units, the simulation does not capture the laminar sublayer, and is offset below the log law by 6 friction velocities.

accuracy for predicting film cooling performance. The simulated boundary-layer shape factor is within $2 \%$ of the accepted fully-turbulent value. The realistic outer velocity profile and resolved log law gradient suggest that resolution away from the wall is adequate, and it is hypothesised that the inner boundary layer, height $y / D \leq 0.16$, is not thick enough to affect the coolant jet trajectory, height of order $y / D \sim 1$.

The height of the simulated boundary-layer trip is chosen to yield a boundary-layer thickness to match the experimental conditions. The report of film effectiveness data by Saumweber et al. (2003) does not contain measurements of the main-stream boundary layer. Therefore, the simulated boundary-layer thicknesses are compared to available measurements from other studies conducted on different configurations of the same

Table 7.2 Comparison of measured and simulated upstream boundary-layer properties.

\begin{tabular}{lllll} 
Thickness & Comp. & Expt. & Reference & $x / D$ \\
\hline Displacement, $\delta_{*} / D$ & 0.121 & 0.125 & Saumweber and Schulz $(2012)$ & -1.5 \\
$99 \%$ Velocity, $\delta_{99 \%} / D$ & 0.89 & 0.8 & Thole et al. (1998) & -2.0 \\
Shape Factor, $\delta_{*} / \theta$ & 1.43 & 1.38 & Wittig et al. (1996) & -5.0 \\
\hline
\end{tabular}


experimental apparatus in Table 7.2. As the apparatus is equipped with a boundary layer bleed, in the absence of more detailed information, it is reasonable to assume that the boundary-layer thickness is controlled to similar values (normalised by hole diameter) across all reported studies.

The simulated displacement thickness, shape factor, and 99\% velocity thickness match the experimental values to within $-2.9 \%,+3.4 \%$, and $+11.2 \%$ respectively, showing that the modelled boundary layer is representative of that present in the experiment.

\subsection{Cylindrical hole}

Computations of the cylindrical hole geometry at blowing ratios $B R=0.5$ and $B R=1.5$, as reported by Saumweber et al. (2003), are now presented. The aerodynamics of the flow are discussed first, followed by a comparison of the predicted film effectiveness with experimental data.

\subsubsection{Aerodynamics}

Stagnation temperature fields The behaviour of coolant after it leaves the cooling hole is illustrated using contours of time-averaged stagnation temperature on the hole centre plane, $z / D=0$, in Figure 7.4, plotted as an effectiveness defined,

$$
\Theta=\frac{T_{0}-T_{0 \infty}}{T_{0 \mathrm{c}}-T_{0 \infty}}
$$

such that $\Theta=0$ indicates main-stream fluid, $\Theta=1$ indicates coolant fluid, and on adiabatic walls $\Theta=\varepsilon_{\mathrm{f}}$. From Figure 7.4(a), at $B R=0.5$ coolant remains attached to the surface, with effectiveness monotonically decreasing in $y / D$ for $x / D \geq 3$. From Figure 7.4(b), at $B R=1.5$ the increased momentum of the flow exiting the hole causes coolant to lift off from the surface. For example, on this plane, the peak effectiveness at a streamwise location $x / D=7$ occurs well away from the wall at $y / D \approx 1$.

Velocity fields The time-averaged velocity fields on the hole centre plane for low and high blowing ratios are shown in Figure 7.5. At $B R=0.5$, Figure 7.5(a), the hole velocity is approximately $30 \%$ of the main-stream velocity, and coolant accelerates as it leaves the hole and is 'swept up' by the main stream. At $B R=1.5$, Figure $7.5(\mathrm{~b})$, the hole velocity is close to the main-stream velocity, with a coolant to main stream velocity ratio $V R=0.9$, producing a smaller wake region on interaction with the main stream. 


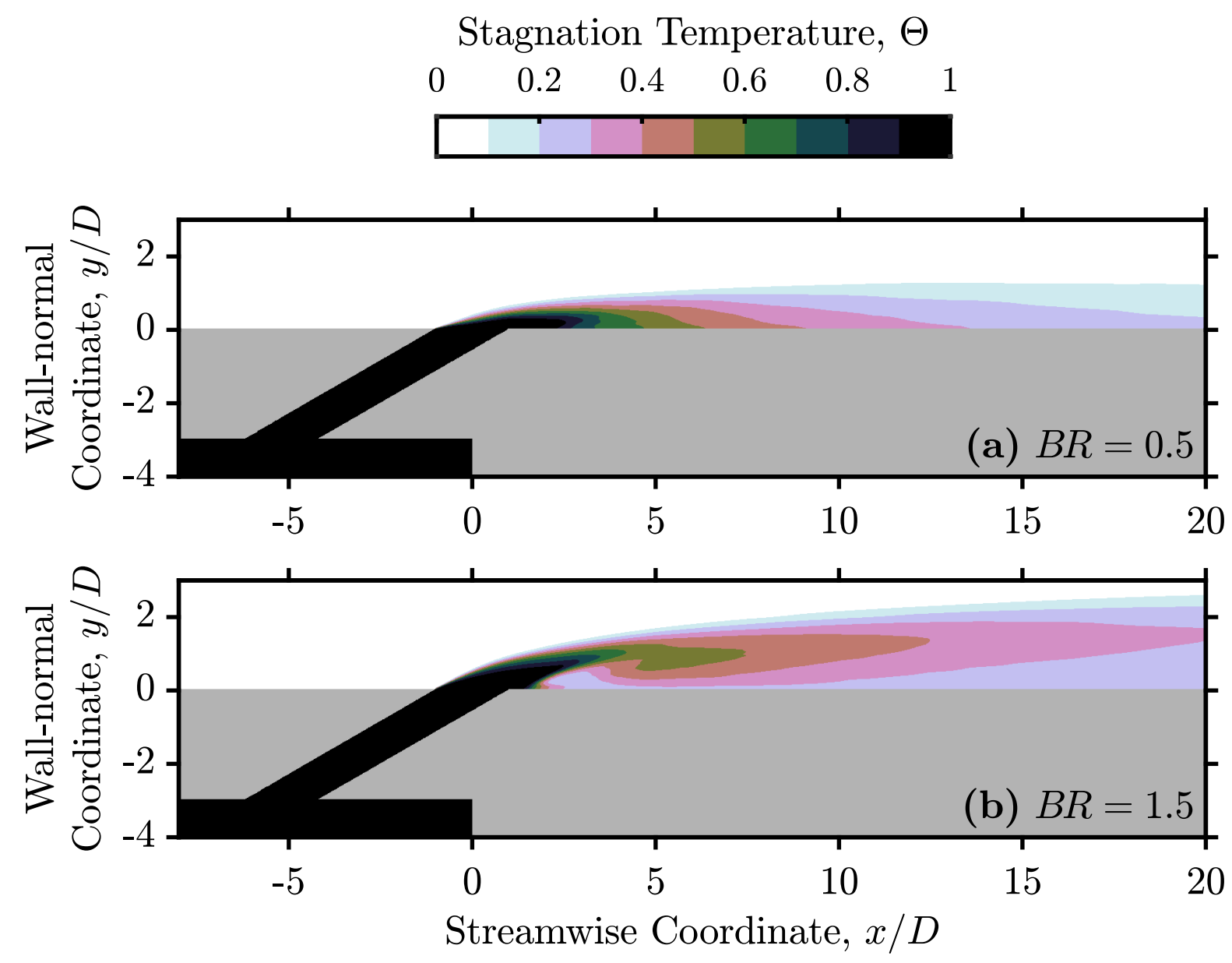

Fig. 7.4 Contours of time-averaged stagnation temperature, cylindrical hole, at hole centre plane, $z / D=0$ : (a) $B R=0.5$, (b) $B R=1.5$. Coolant separates from the wall at high blowing ratio. 


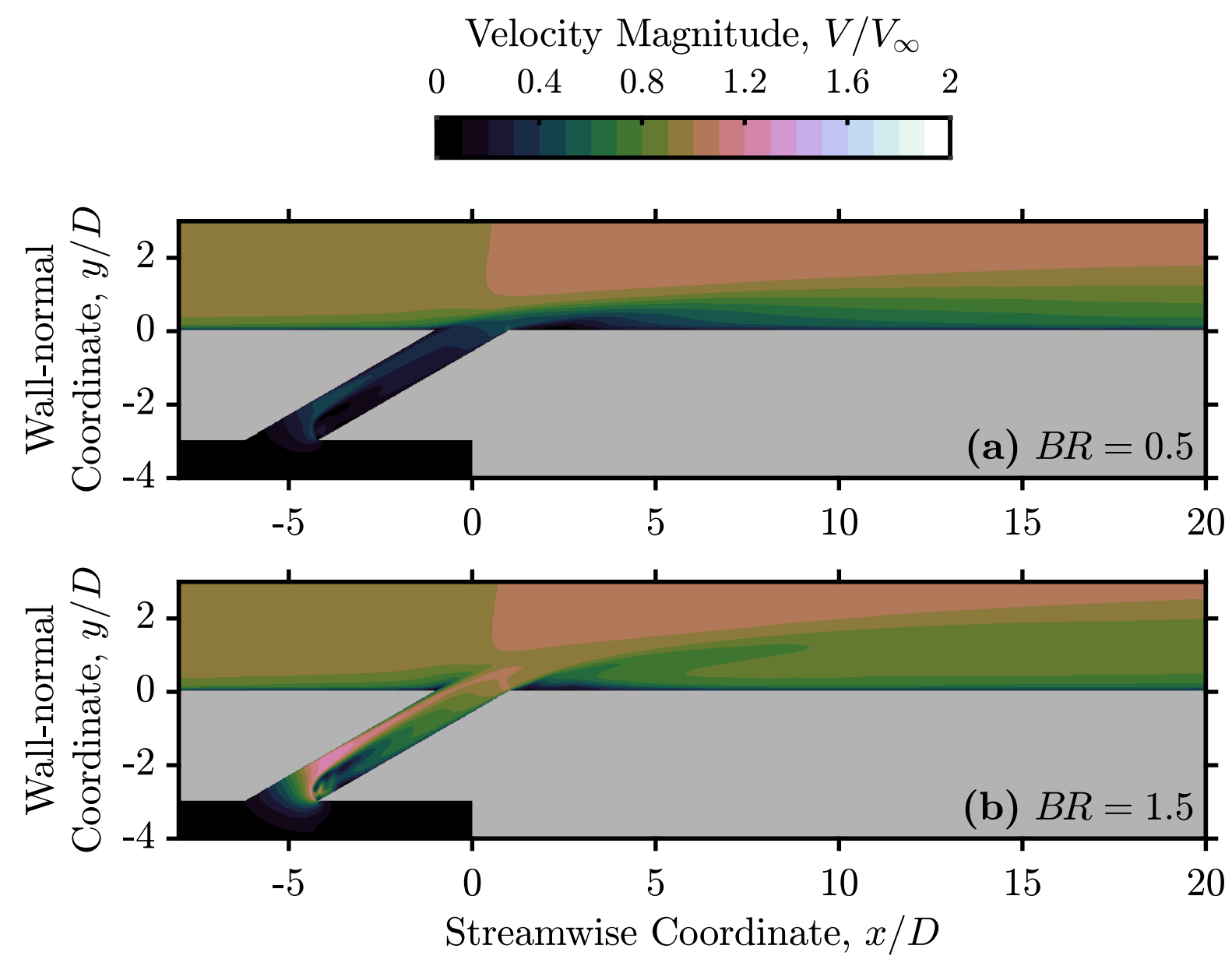

Fig. 7.5 Contours of time-averaged velocity magnitude, cylindrical hole, at hole centre plane, $z / D=0$ : (a) $B R=0.5$, (b) $B R=1.5$. At low blowing ratio, coolant accelerates as it exits the hole; at high blowing ratio, the coolant velocity is close to the main-stream value, with $V R=0.9$, producing a smaller wake region.

Coherent vortical structures A illustration of the turbulence resolved in the simulation is given in the visualisation of coherent vortical structure in Figure 7.6, for an instantaneous flow field of the $B R=1.5$ case. Vortices are identified by calculating an isosurface of positive second invariant of the velocity gradient tensor, or 'Q-criterion', after Hunt et al. (1988). The isosurface is coloured by local stagnation temperature, such that main-stream structures are yellow and coolant structures are blue. The separation over the trip at $x / D=-14$ and immediate transition to turbulence is evident, with hairpin vortices visible in the downstream boundary layer. There is increased vortical activity at the interaction between coolant and main stream at $x / D \approx 0$. 


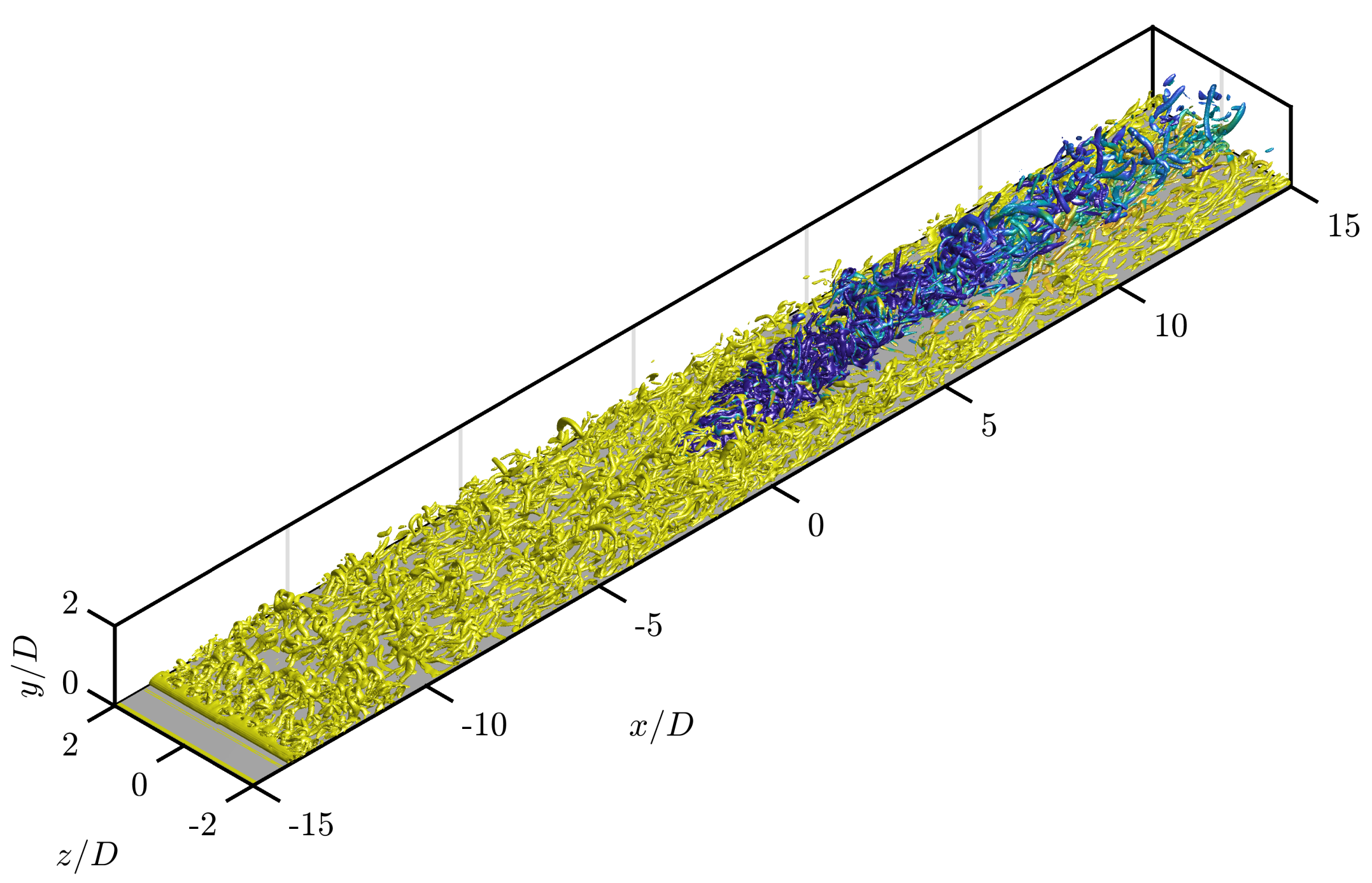

Fig. 7.6 Vortical structures identified by isosurface of positive Q-criterion, coloured by local stagnation temperature. Instantaneous snapshot, cylindrical hole at $B R=1.5$. 


\subsubsection{Heat transfer}

Two-dimensional film effectiveness distributions Coloured contours of predicted film effectiveness over the cooled surface, the $y / D=0$ plane, are shown for low and high blowing ratios in Figure 7.7. Overlaid are line contours as presented by Saumweber et al. (2003), allowing a comparison of the measured and simulated distributions. At both blowing ratios, the coverage of coolant, as defined by the lateral extent of an $\varepsilon_{\mathrm{f}}=0.1$ contour, is predicted to within $0.1 D$. At $B R=0.5$, in Figure 7.7(a), the local film effectiveness is over-predicted by up to 0.1 near the hole, but the contour shape is similar to the experiment. At $B R=1.5$, Figure 7.7(b), the contour resolution is too coarse to make a detailed comparison. The predicted two-dimensional film effectiveness distributions are in qualitative agreement with the experiment.

Lateral film effectiveness distributions A known deficiency of RANS turbulence models applied to film cooling is a failure to predict lateral spreading of coolant downstream of ejection, Section 2.5. The ability of the present URANS-LES method to do so is assessed in Figure 7.8, comparing lateral distributions of film effectiveness across the hole pitch with experimental data. At $x / D=5$, Figure $7.8(\mathrm{a})$, there is a tendency for increasing over-prediction towards the centreline, by up to 0.05 for $z / D=-0.25, B R=0.5$. There is asymmetry in the experimental data at both blowing ratios, which is not present in the computation. Further downstream of the hole at $x / D=15$, Figure $7.8(\mathrm{~b})$, similar trends are observed. The present computations under-predict lateral spreading, which could be attributed to the lack of main-stream turbulence, but represent an improvement over RANS models. For example, the RANS simulations of Walters and Leylek (1997) under-predicted lateral coolant coverage by up to $47 \%$.

Laterally-averaged film effectiveness The key input required by a cooling designer is the streamwise distribution of laterally-averaged film effectiveness, in order to predict the total heat load on the blade surface, so quantitative predictions are of engineering importance. Figure 7.9 compares the predicted streamwise distributions of laterallyaveraged film effectiveness with the available experimental data. Flow unsteadiness results in uncertainty in quantities averaged over a finite time. Here, the numerical solution is averaged over ten flow-through times, and 95\% confidence intervals are calculated using individual averages from five restarts and shaded on Figure 7.9. At $B R=0.5$, the computations over-predict laterally-averaged film effectiveness by between $+11 \%$ and $+15 \%$ at all streamwise locations. This is explained by the lack of main-stream turbulence in the present computations; extrapolating the data of Saumweber et al. 


\section{Film Effectiveness, $\varepsilon_{\mathrm{f}}$}
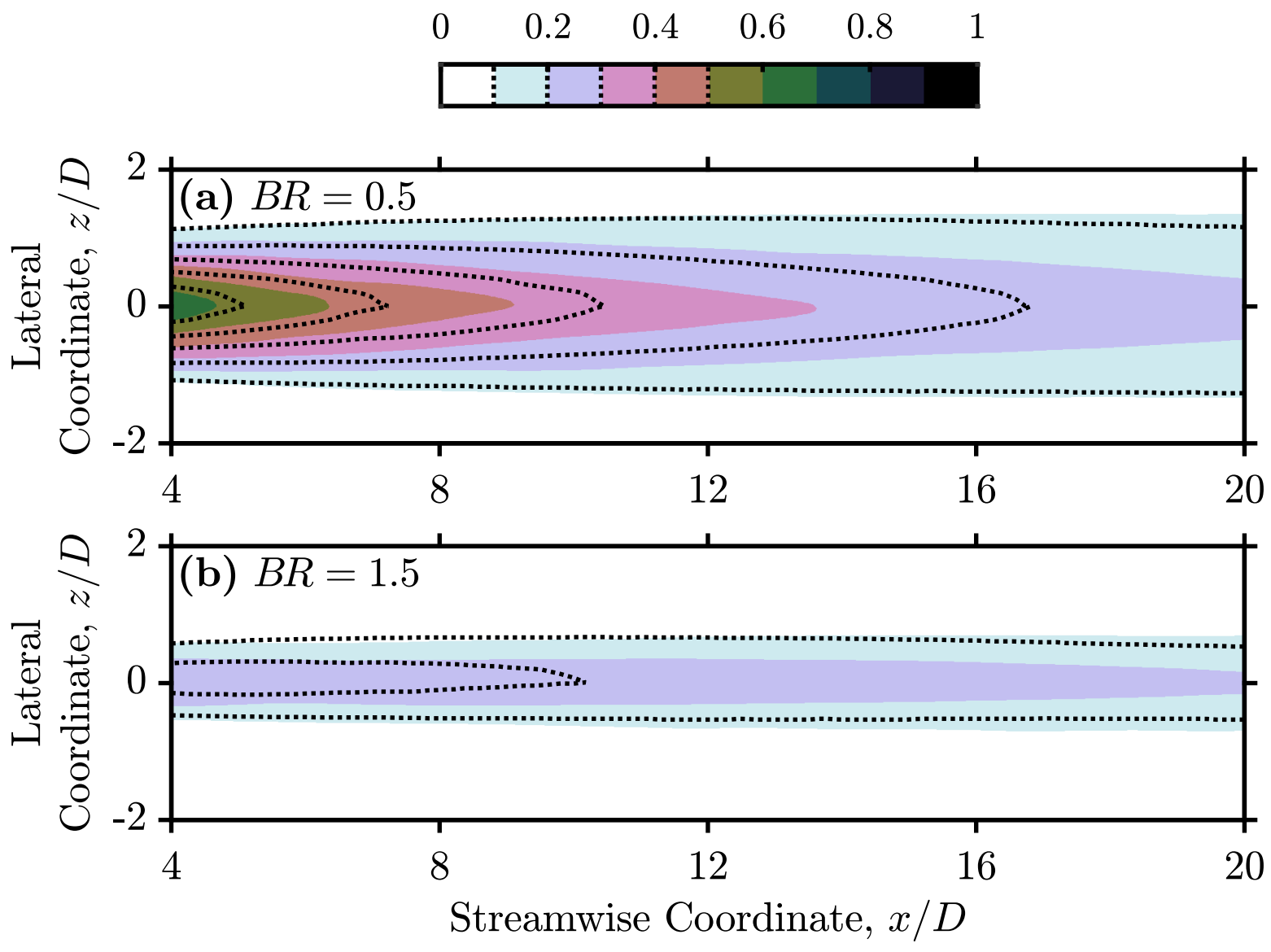

Fig. 7.7 Contours of time-averaged local film effectiveness, cylindrical hole, on wall at $y / D=0$, with overlaid dashed line contours measured by Saumweber et al. (2003), cases: (a) $B R=0.5$, (b) $B R=1.5$. Lateral coolant coverage is predicted to within $0.1 D$, and the local distribution shapes are in qualitative agreement with experiment.

(2003) at $T u=3.6 \%$ and $7.5 \%$ to $T u=0 \%$ suggests an over-prediction of around $+10 \%$ should be expected. The agreement between computation and experiment is within $+7 \%$ to $+21 \%$ at $B R=1.5$, when a similar extrapolation to $T u=0 \%$ yields an expected error in the opposite direction of $-10 \%$ to $-24 \%$. The maximum absolute error in laterally-averaged film effectiveness at this condition is 0.014 . In addition to main-stream turbulence effects, a greater discrepancy at high blowing ratio may be expected because resolution requirements, in both space and time, are greater at this condition. Smallerscale turbulence is generated by higher levels of shear and mixing with a separated coolant jet, which must then be convected at a higher bulk coolant velocity. Overall, given the known challenge of simulating high momentum flux ratio cases (Bogard and 


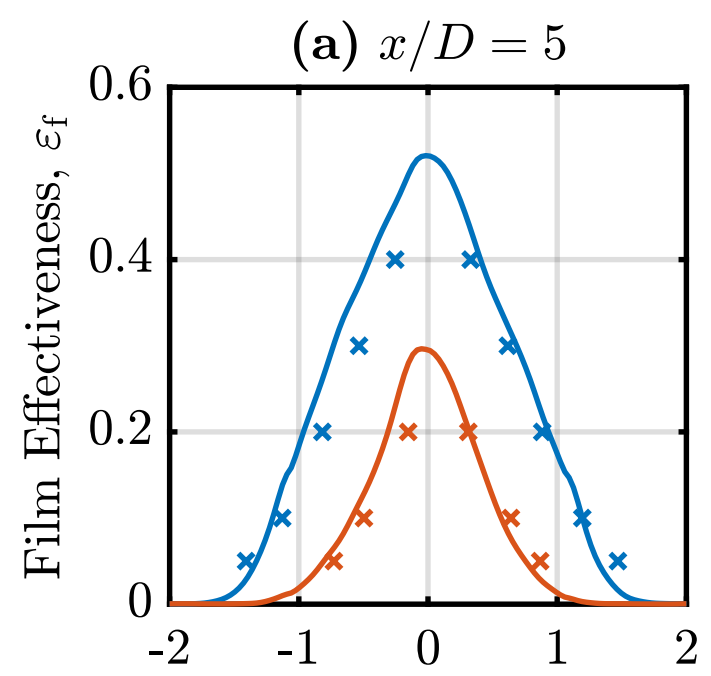

Lateral Coordinate, $z / D$

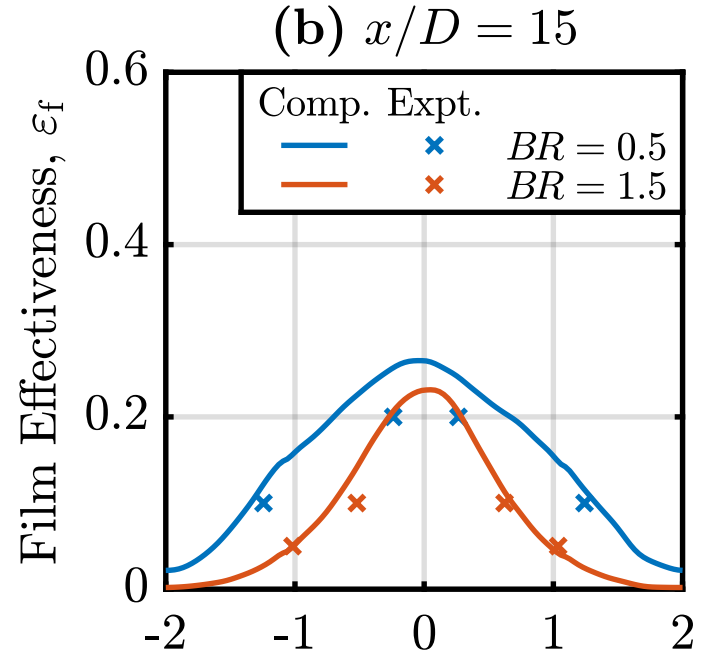

Lateral Coordinate, $z / D$

Fig. 7.8 Lateral distributions of time-averaged local film effectiveness, cylindrical hole, compared to the measurements of Saumweber et al. (2003) at streamwise locations: (a) $x / D=5$, (b) $x / D=15$. The computations over-predict effectiveness towards the hole centreline, by up to 0.05 .

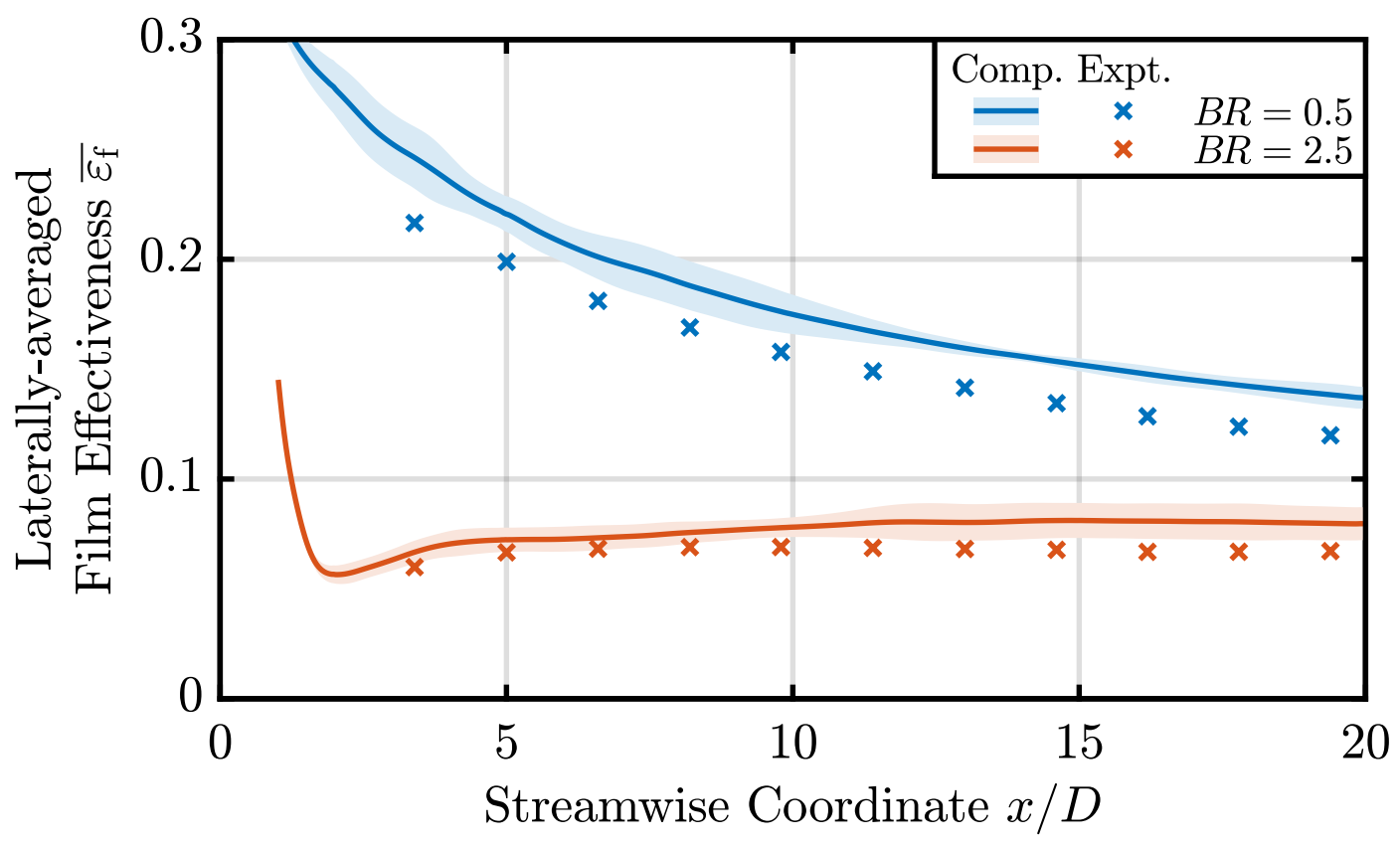

Fig. 7.9 Streamwise distributions of time-averaged, laterally-averaged film effectiveness, cylindrical hole, compared to measurements of Saumweber et al. (2003) blowing ratios: (a) $B R=0.5$, (b) $B R=1.5$. The predictions are accurate to within $+7 \%$ to $+21 \%$. 
Thole, 2006), and modelling and experimental uncertainties, the present approach yields state-of-the-art predictions of laterally-averaged film effectiveness.

URANS layer sensitivity The numerical method relies on a URANS layer, of constant thickness $\delta_{l}$, to capture the inner region of the boundary layer without direct resolution of the fine turbulent structures there. The value of $\delta_{l}$ sets the boundary between URANS and resolved LES zones. In all simulations presented up to now, a fixed thickness $\delta_{l} / D=8 \%$ has been used, but in practice $\delta_{l}$ is a free parameter chosen a-posteriori. Restricting the URANS layer to the universal log law region requires, at the Reynolds number of the present case, $4 \% \leq \delta_{l} / D \leq 12 \%$. Figure 7.10 shows the variation in area-averaged film effectiveness prediction error, $\Delta \bar{\varepsilon}_{\mathrm{f}}(A)$, as $\delta_{l}$ is varied around this range. The film effectiveness is area-averaged over $3 \leq x / D \leq 20$ and $-2 \leq z / D \leq 2$; both absolute and relative errors are plotted in Figures 7.10(a) and 7.10(b) respectively. In absolute terms, the $B R=1.5$ case is less sensitive to $\delta_{l}$ than the $B R=0.5$ case, because the coolant jet is separated and the near-wall modelling exerts less influence on the total mixing between coolant and main-stream. However, in relative terms, the $B R=1.5$ case is more sensitive because the value of film effectiveness is lower. For both blowing ratios, the smallest discrepancy with respect to the experimental data is $7 \% \leq \delta_{l} / D \leq 8 \%$, when the turbulent viscosity falls to zero for $y^{+} \geq 125$. Favourably, the prediction accuracy is not sensitive to the precise value of $\delta_{l}$, and errors remain below 0.02 or $20 \%$ for all values $\delta_{l} / D \geq 6 \%$.

\subsection{Shaped hole}

Computations of the shaped hole geometry at blowing ratios $B R=0.5$ and $B R=2.5$, as reported by Saumweber et al. (2003), are now presented. The simulation parameters, including the URANS layer thickness, are unchanged from the cylindrical hole case, testing the predictive capability of the method on a new geometry. The aerodynamics of the flow and film effectiveness results are presented in turn.

\subsubsection{Aerodynamics}

Stagnation temperature fields Figure 7.11 shows contours of time-averaged stagnation temperature for the shaped hole at low and high blowing ratios. At low blowing ratio, Figure 7.11(a), the penetration of coolant into the main stream is reduced compared to a cylindrical hole operating at the same blowing ratio, in Figure 7.4(a). At a fixed streamwise location $x / D=5$, the wall-normal extent of the $\varepsilon_{\mathrm{f}}=0.1$ contour reduces by $30 \%$, 

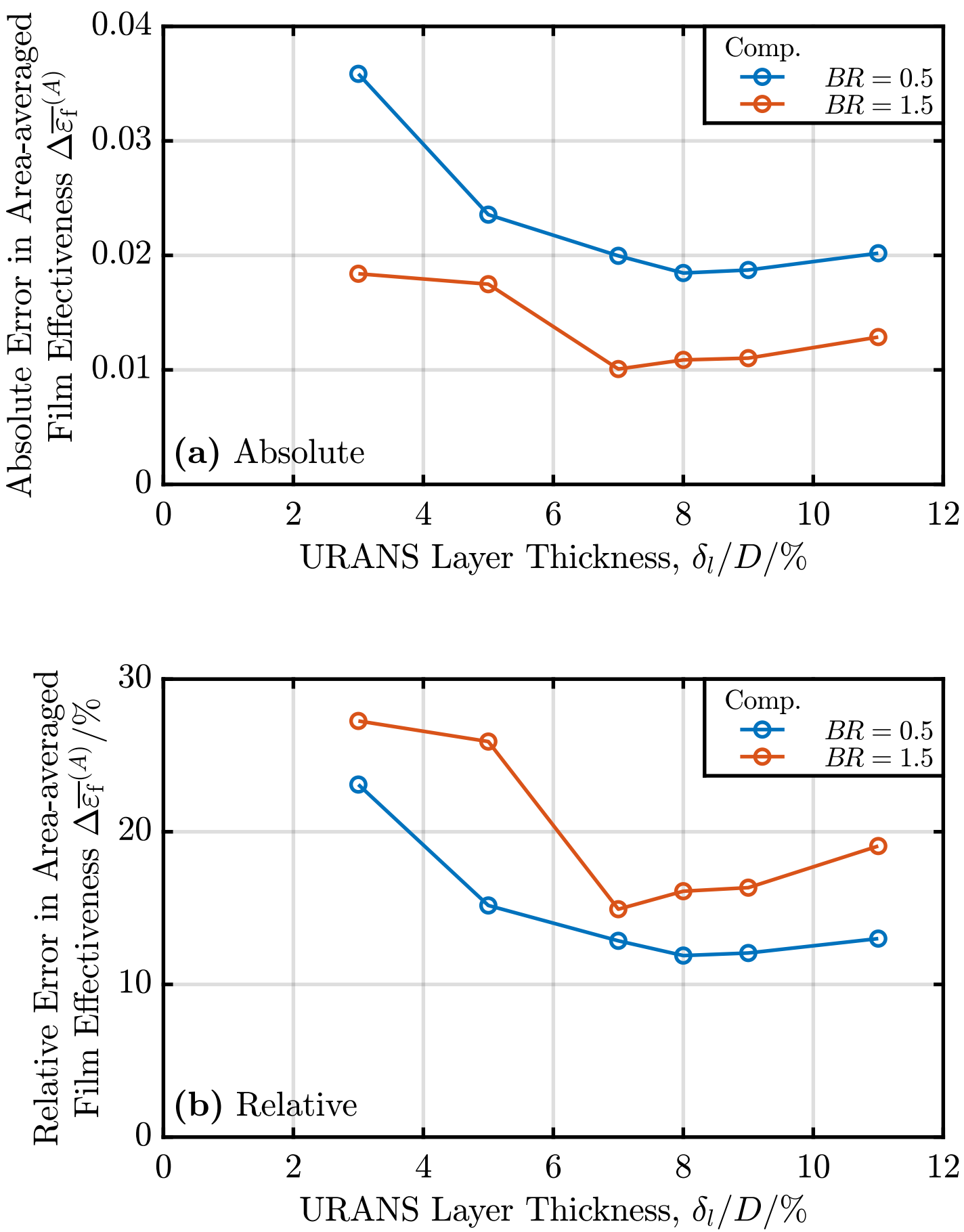

Fig. 7.10 Effect of URANS layer thickness on film effectiveness prediction accuracy, cylindrical hole, area-averaged error for $3 \leq x / D \leq 20,-2 \leq z / D \leq 2$ : (a) absolute error; (b) relative error. The error for both blowing ratios is insensitive to URANS layer thickness for $\delta_{l} / D \geq 6 \%$. 


\section{Stagnation Temperature, $\Theta$

$\begin{array}{llllll}0 & 0.2 & 0.4 & 0.6 & 0.8 & 1\end{array}$
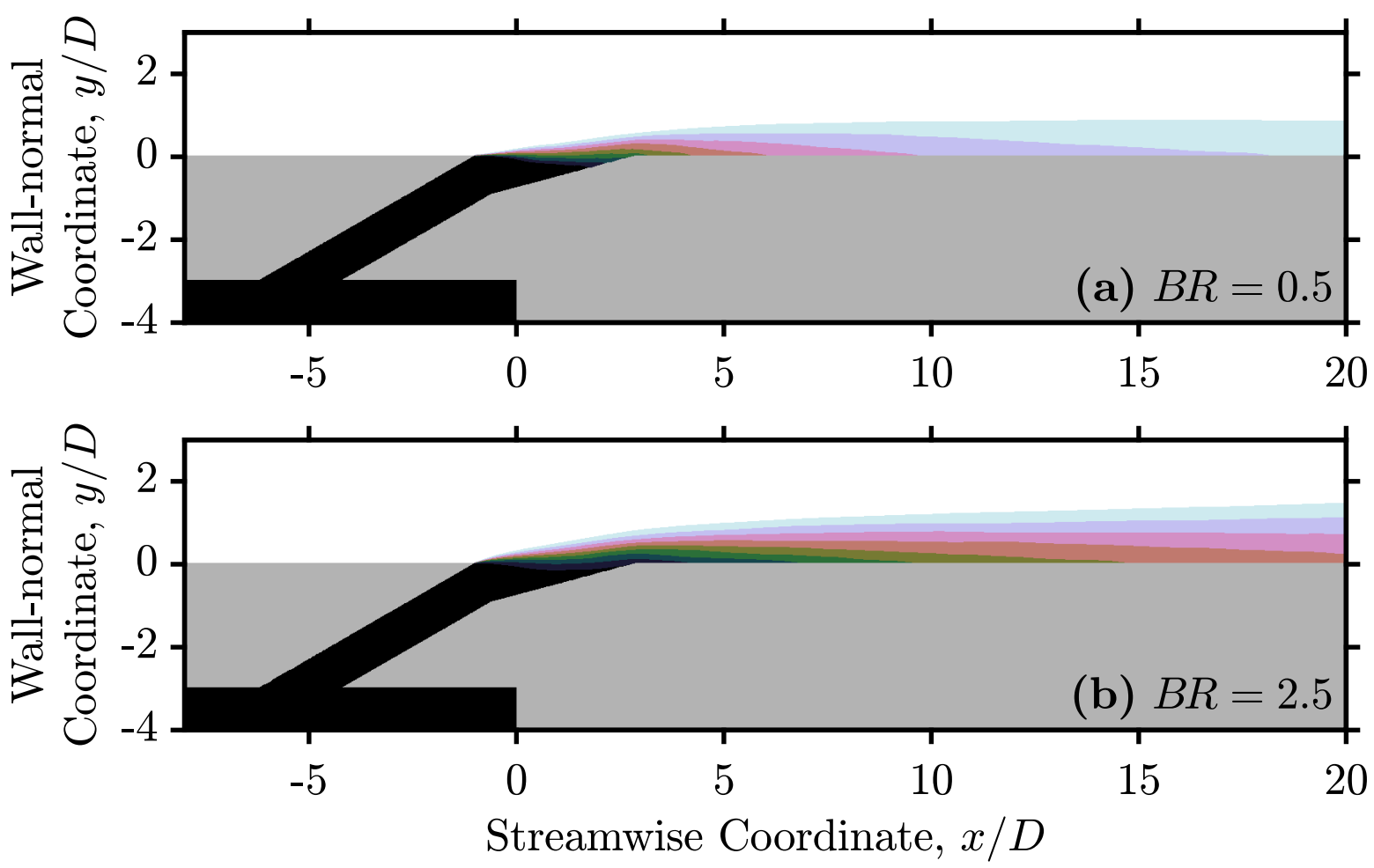

Fig. 7.11 Contours of time-averaged stagnation temperature, shaped hole, at hole centre plane, $z / D=0$ : (a) $B R=0.5$, (b) $B R=2.5$. Coolant remains attached to the wall at high blowing ratio.

from $y / D=1.0$ in the cylindrical case, to $y / D=0.7$ in the shaped case. This is because coolant momentum is reduced by the diffusing hole shape. Reduced momentum also causes coolant to exit from the downstream side of the hole opening. At the high blowing ratio, Figure 7.11(b), coolant remains attached to the surface, unlike a cylindrical hole, Figure 7.4(b), with effectiveness monotonically decreasing in the wall-normal direction.

Velocity fields The contours of time-averaged velocity magnitude at $B R=0.5$ in Figure 7.12 (a) show that a shaped hole reduces the velocity at the cooling hole exit, $V / V_{\infty} \approx 0.2$, compared to a cylindrical hole, $V / V_{\infty} \approx 0.4$ in Figure $7.5(\mathrm{a})$. At high blowing ratio, Figure 7.12(b), the velocity in the metering section of the shaped hole reaches supersonic levels as it accelerates past the blockage of the hole inlet separation. 


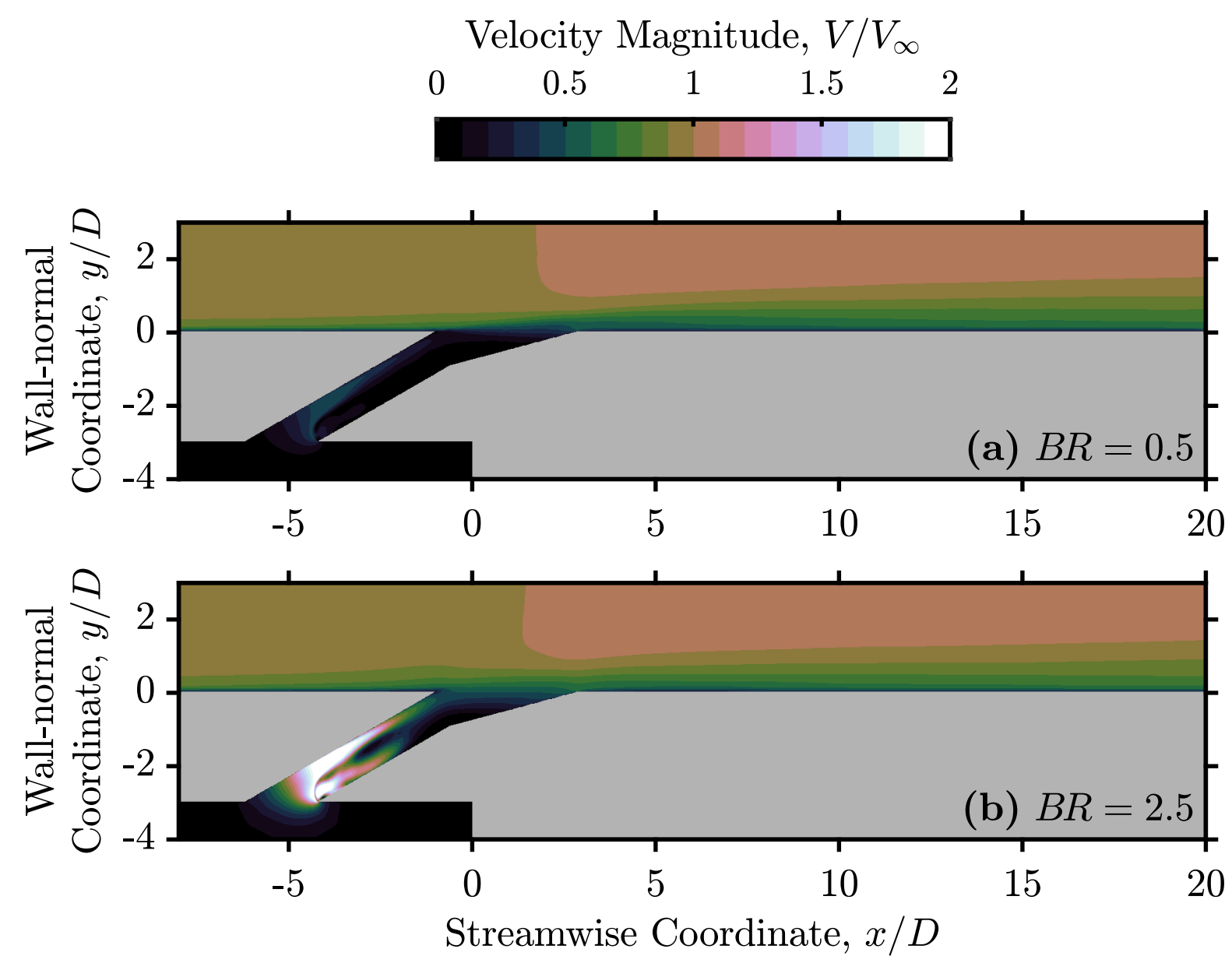

Fig. 7.12 Contours of time-averaged velocity magnitude, shaped hole, at hole centre plane, $z / D=0$ : (a) $B R=0.5$, (b) $B R=2.5$. Flow accelerates to supersonic in the hole metering section at $B R=2.5$, due to inlet separation blockage.

The expanding outlet section does not encourage flow to reattach to the downstream side of the hole, where a low-velocity recirculation is formed.

Discharge coefficient The cooling hole discharge coefficients, for both the shaped and cylindrical geometries, are compared with experimental measurements by Saumweber et al. (2003) in Figure 7.13. The discharge coefficient is under-predicted by $4 \%$ to $5 \%$ in the cylindrical hole case, and over-predicted by up to $7 \%$ in the shaped hole case. Saumweber et al. (2003) quote an uncertainty in discharge coefficient of between $2 \%$ and $4.5 \%$. This level of agreement suggests that separations and blockage within the holes are well-modelled in the present computations. 


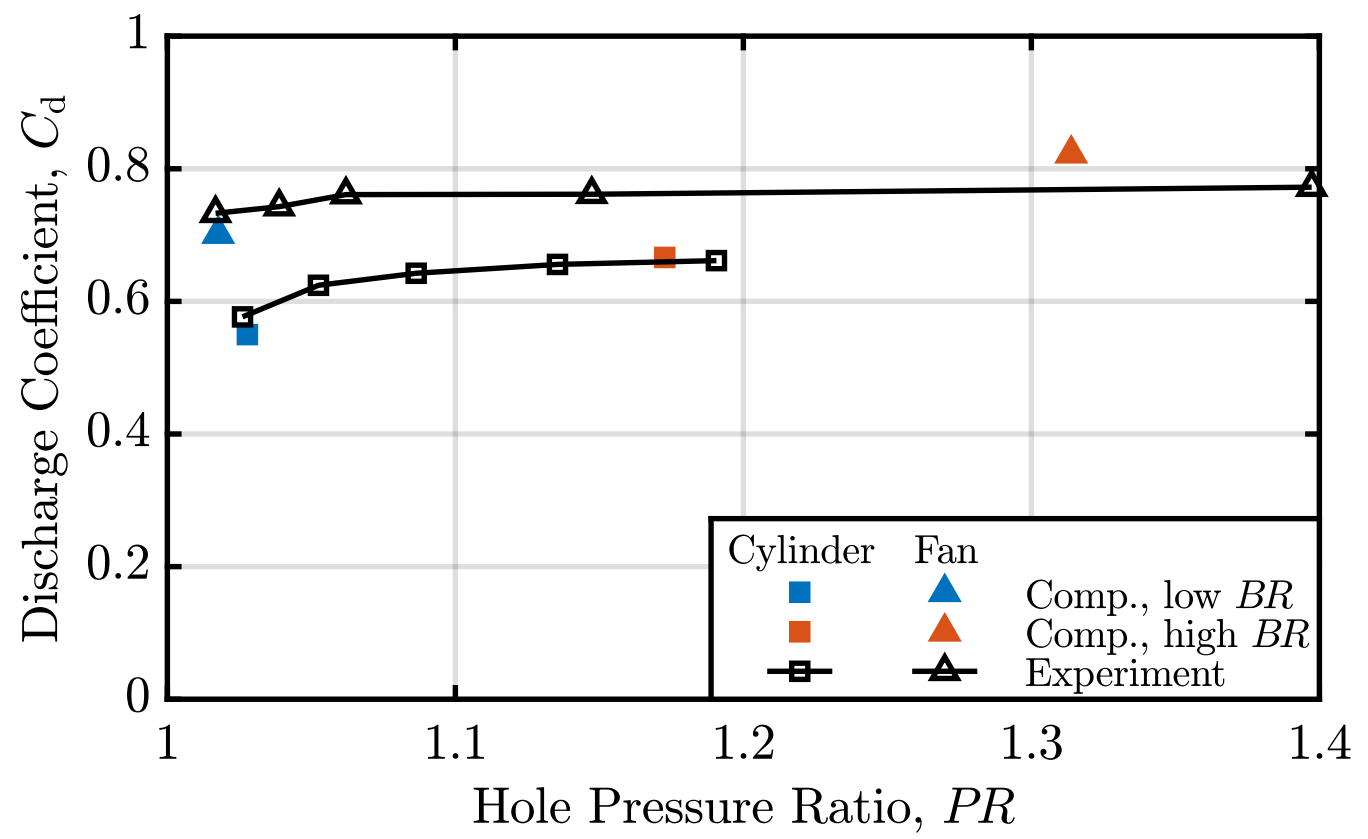

Fig. 7.13 Discharge coefficients for all computational cases compared to experimental data of Saumweber et al. (2003). The predictions are accurate to within $\pm 7 \%$.

\subsubsection{Heat transfer}

Two-dimensional film effectiveness distributions Computationally-predicted film effectiveness values for the shaped hole over the plane $y / D=0$ are shown as coloured contours in Figure 7.14. Overlaid as line contours are the corresponding experimental results. The expanded exit of the shaped hole provides complete coolant coverage, with $\varepsilon_{\mathrm{f}} \geq 0.1$ over the entire plane. This leads to low spatial gradients in film effectiveness, and sparse contour lines in the reported experimental data, making the spatial location of the contours sensitive to errors in film effectiveness. At $B R=0.5$, in Figure 7.14(a), the computation does not show the double-peak, 'bimodal' lateral distribution of effectiveness as in the experiments at $5 \leq x / D \leq 7$. This could be attributed to the sensitivity of diffusers to geometry and inlet conditions: any small inaccuracies will affect the hole exit flow field. At $B R=2.5$, in Figure 7.14(b), a bimodal pattern is evident in both the computational and experimental results across all streamwise locations, indicating separation within the cooling hole on the downstream wall of the diffuser (Saumweber and Schulz, 2012). 
Film Effectiveness, $\varepsilon_{\mathrm{f}}$
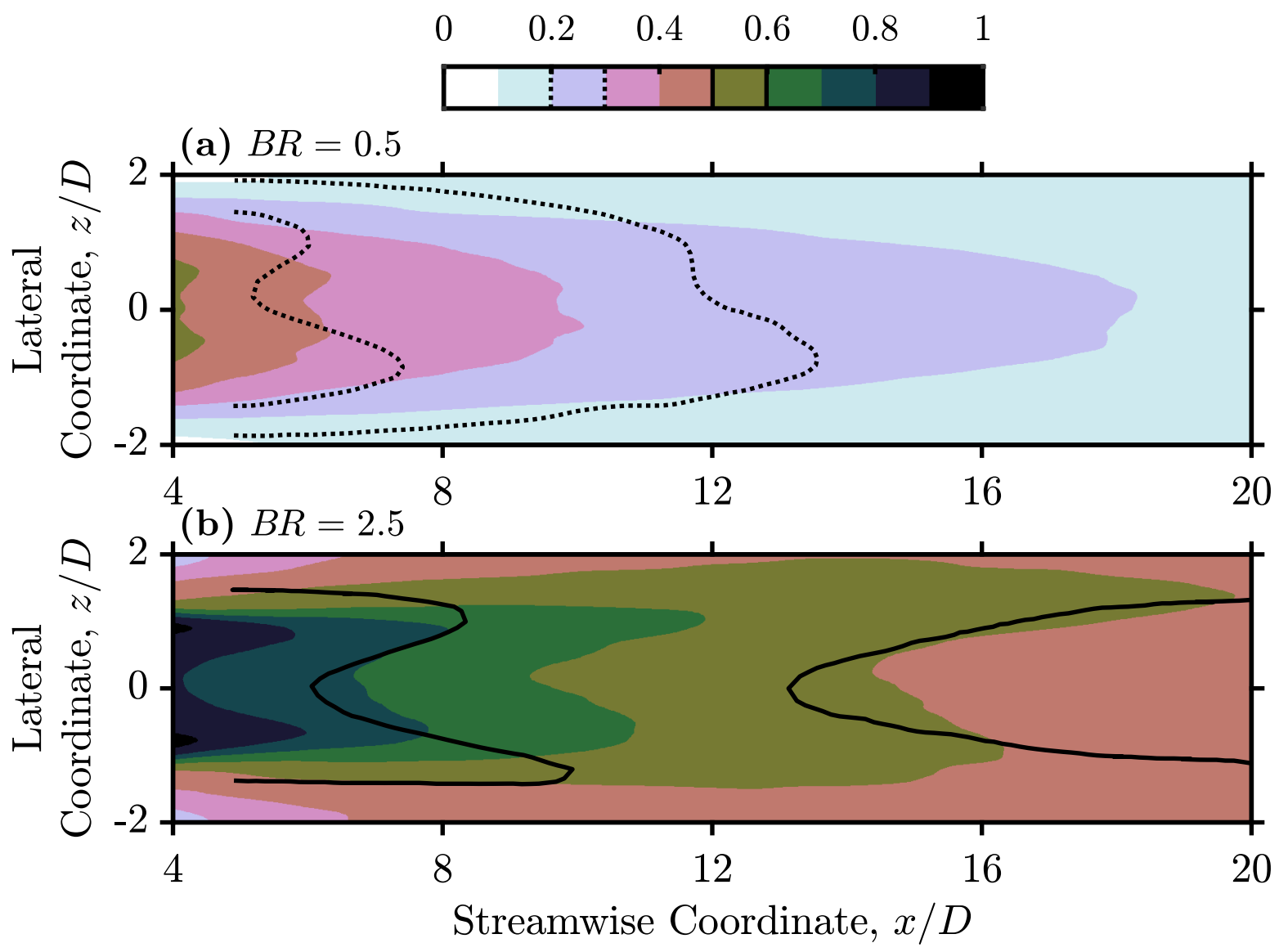

Fig. 7.14 Contours of time-averaged local film effectiveness, shaped hole, on wall at $y / D=0$, with overlaid dashed line contours measured by Saumweber et al. (2003), blowing ratios: (a) $B R=0.5$, (b) $B R=2.5$. Both computation and experiment at $B R=2.5$ exhibit double-peak lateral distributions of effectiveness. 


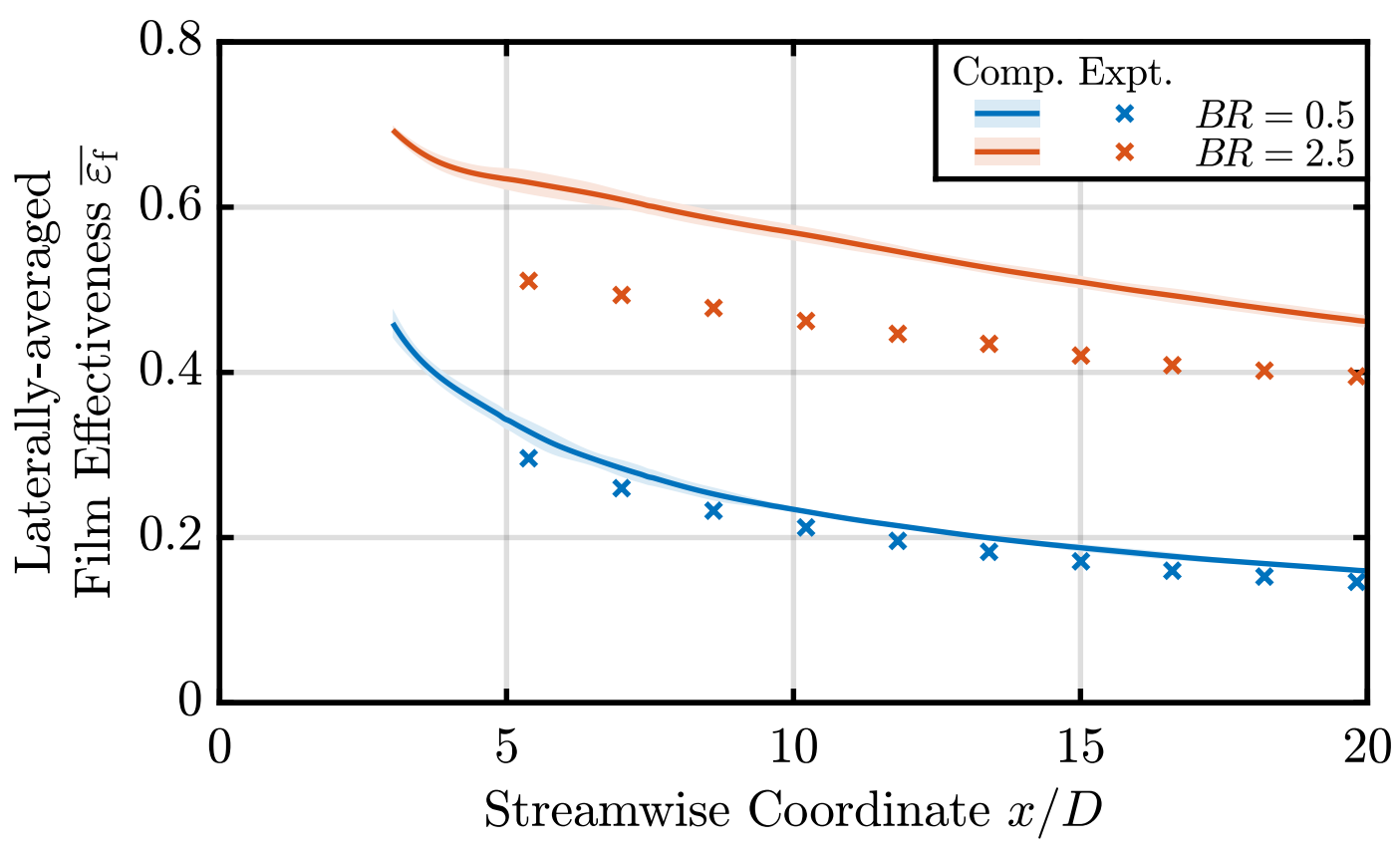

Fig. 7.15 Streamwise distributions of time-averaged, laterally-averaged film effectiveness, shaped hole, compared to the measurements taken by Saumweber et al. (2003), blowing ratios: (a) $B R=0.5$, (b) $B R=2.5$. The predictions are accurate to within $+9 \%$ to $+23 \%$.

Laterally-averaged film effectiveness distributions Figure 7.15 compares predicted laterally-averaged film effectiveness distributions with the experimental data for laid-back fan-shaped holes. At $B R=0.5$, the predictions are accurate to between $+9 \%$ and $+11 \%$ at all streamwise locations. As with the cylindrical hole case, this is consistent with the lack of simulated main-stream turbulence, which by extrapolating the data of Saumweber et al. (2003) would be expected to produce an over-prediction of about $+10 \%$. The prediction accuracy at $B R=2.5$ is reduced, with errors approximately double those of the low blowing ratio case: an over-prediction of between $+17 \%$ and $+23 \%$. It is hypothesised that refining the time-step would improve these results. The time step is set by a fixed $C F L=0.5$, defined in the main stream based on the streamwise grid spacing and main-stream velocity, Equation (4.1). This ensures four points per wavelength of the smallest disturbance resolvable on the spatial discretisation in this region. However, Figure 7.12(b) shows that coolant accelerates to over twice the main-stream velocity within the hole, and a smaller time-step may be required to properly resolve this region at such a high velocity ratio. A deficiency in resolution of the hole flow at this condition is consistent with the largest error in discharge coefficient of $7 \%$ also occurring at this condition. 


\subsection{Conclusions}

The hybrid URANS-LES simulations discussed in this Chapter comprise a validation of film effectiveness predictions using a consistent high-fidelity computational model across multiple film cooling geometries and operating conditions. The following conclusions may be drawn from the presented results:

1. The simulations, using uniform inflow over a trip, produce a fully-developed, equilibrium turbulent boundary layer at the cooling hole location. The velocity profile in the outer region of the boundary layer is realistic, but the mixing-length wall model does not capture the laminar sublayer. Based on the data available, the boundary-layer thickness is representative of the experimental conditions.

2. For both cylindrical and shaped holes, computationally-predicted flow features are in qualitative agreement with the film cooling literature. Lateral spreading is modelled better than in RANS computations, with lateral coolant coverage predicted to within $0.1 D$ in the cylindrical hole case.

3. The computational results for laterally-averaged film effectiveness downstream of cylindrical holes are accurate to within $+15 \%$ at low blowing ratio, $B R=0.5$, and to within $+21 \%$ at high blowing ratio, $B R=1.5$. Over-prediction at low blowing ratio is consistent with the lack of main-stream turbulence in the present simulations. The discrepancy at $B R=1.5$ is in the opposite direction to the expected main-stream turbulence effect. Given the accepted challenge of modelling lifted-off coolant, these predictions are state-of-the-art.

4. For a cylindrical hole, predictions of film effectiveness are insensitive to URANS layer thickness $\delta_{l}$ : the area-averaged error with respect to experiment remains below $20 \%$ at both low and high blowing ratio when $\delta_{l} / D \geq 6 \%$. This suggests that, when simulating a new case, the results will not be a strong function of the choice of URANS layer thickness.

5. Applying the same computational model, as calibrated using the cylindrical hole, to a shaped hole also produces predictions of laterally-averaged film effectiveness with similar accuracy. The computations are accurate to within $+11 \%$ at low blowing ratio, $B R=0.5$, and to within $+23 \%$ at high blowing ratio, $B R=2.5$. This agreement, without additional calibration, increases confidence in the general predictive capability of the approach. 



\title{
Chapter 8
}

\section{Film-resolved cascade simulations}

\begin{abstract}
The hybrid URANS-LES computational method, validated in Chapter 7, is applied to a turbine stage linear cascade with resolved rotor film cooling holes. The geometry and boundary conditions are representative of the mid-span of the first high-pressure turbine stage of a large industrial gas turbine. Two computational model configurations with different rotor-stator interfaces are compared: a mixing plane, which eliminates blade row interaction; and a sliding plane, which includes blade row interaction.

The results allow quantification of the effect of blade row interaction on rotor film cooling performance. Compared to steady inflow conditions, film effectiveness is reduced by up to 0.04 or $18 \%$ on the pressure side, and up to 0.08 or $30 \%$ on the suction side.

The pressure-side deficit is attributed to vane coolant migration. The suction-side deficit is attributed to wake interaction periodically increasing the film mixing rate, by a factor of 2.5. Ensemble-averaged data show that film effectiveness recovers to the mixing-plane, zero-turbulence value between wake passings.

Comparison of resolved unsteady momentum flux ratio with predictions using the quasi-steady hole response model from Chapter 6 shows good agreement, typically within $5 \%$, validating the model and its assumptions.
\end{abstract}




\subsection{Introduction}

In this Chapter, the hybrid URANS-LES computational method described in Chapter 4, and validated in Chapter 7, is applied to a linear cascade model of the mid-span of a high-pressure turbine stage, representative of a large industrial gas turbine. Resolution of rotor cooling holes allows quantitative predictions of instantaneous film effectiveness. The aims of the simulations are to quantify the effect of blade row interaction on film effectiveness in a case with realistic geometry and boundary conditions, and to investigate the flow field in more detail than is possible in rotating-stage experiments.

Two configurations of the cascade model are simulated and compared back-to-back in a virtual experiment. The first configuration has a mixing plane between the stator and rotor rows, such that the flow entering the latter is uniform and hence steady in the relative frame. This case, which excludes blade row interaction, shall be denoted MP for 'mixing-plane'. The second configuration of the computational model uses a sliding plane at the stator-rotor interface, where a circumferential interpolation procedure is used to match flow properties between the grids at each time step. This case, which captures blade row interaction, will be denoted $S P$ for 'sliding-plane'. The two cases have identical geometry, flow solver, and boundary conditions, so their comparison isolates the effect of blade row interaction more precisely than is possible in experimental measurements.

Locations of the meshed rotor cooling holes are shown in Figure 8.1. The blade surface is parametrised using a surface coordinate $\zeta$, where $\zeta=0$ is the leading edge, $\zeta<0$ is the pressure side, and $\zeta>0$ is the suction side, normalised by the blade axial chord $c_{x}$. There are three cooling holes on the leading-edge showerhead. These are: SH1, at $\zeta / c_{x}=0 ; \mathrm{SH} 2$, at $\zeta / c_{x}=-0.04$; and $\mathrm{SH} 3$, at $\zeta / c_{x}=-0.08$. The suction-side cooling hole SS is located at $\zeta / c_{x}=0.41$. The two cooling holes located on the pressure side, $\mathrm{PS} 1$ and PS2, are located at $\zeta / c_{x}=-0.17$ and $\zeta / c_{x}=-0.99$ respectively.

The Chapter is organised into four sections. In the first section, convergence and adequate resolution of the flow field is established. The second section concerns timeaveraged flow field results, including a quantification of the blade row interaction effect on time-averaged cooling performance. The third section analyses the unsteady flow field with reference to the physical mechanisms of interaction described in Chapter 6 of this thesis. Finally, the last section considers the results within the quasi-steady framework established in Chapters 5 and 6. 


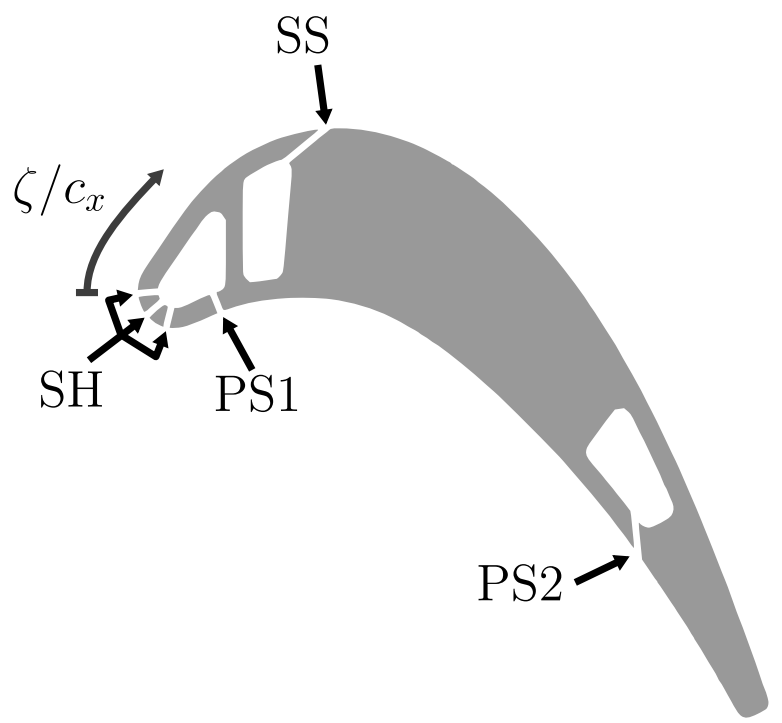

Fig. 8.1 Cooling hole locations and surface coordinate for rotor blade cascade.

\subsection{Convergence and resolution assessment}

As discussed in Section 4.5, convergence is accelerated by starting computations using a solution interpolated from a coarser mesh, and progressively refining the temporal resolution using dual time stepping. In this section, data are presented to demonstrate convergence at all scales and adequate resolution of the flow features of interest.

\subsubsection{Turbine operating point}

At the overall turbine scale, convergence is reached when the operating point, i.e. mass flow or, non-dimensionally, flow coefficient, is steady. A convergence history of the $S P$ cascade inlet mass flow is shown in Figure 8.2. The mass flow is normalised by a reference mass flow, $\dot{m}_{\text {in,ref }}$, time-averaged over $f t \geq 16$. At $f t=0$, the computation is started from an initial guess interpolated from a URANS solution, using coarse time steps with $\Delta t V_{\infty} / D \leq 0.2$ at the cooling holes. The circles indicate 'restarts', where the solution is written out and the computation resumed in a new cluster job.

The mass flow settles down after $f t \approx 6$, giving an approximate time constant of two vane passing periods. The same time constant is observed in the MP simulation. The computations are continued for ten further vane passing periods to verify convergence of the cascade operating point. At $f t=16$, the time step is reduced to the fine value $\Delta t V_{\infty} / D \leq 0.02$ to resolve the film cooling holes. The coarse time step was selected to adequately resolve the vane wake, and consequently the effect of further refining the time step on the cascade operating point is small in both model configurations. For $f t \geq 16$, 


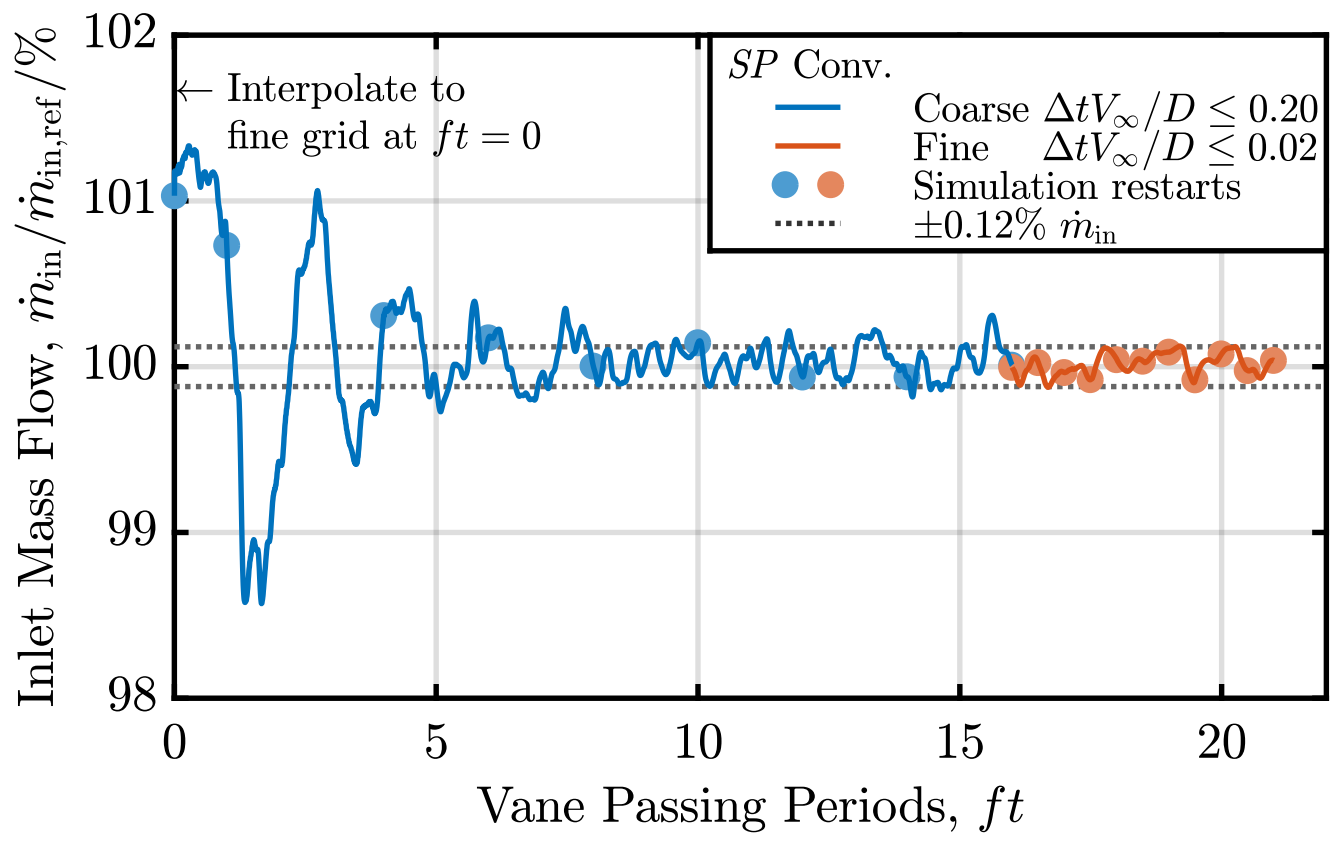

Fig. 8.2 Convergence history of film-resolved $S P$ cascade inlet mass flow. The settling time constant is of order two vane passing periods. Inlet mass flow remains constant to within $\pm 0.12 \%$ for $f t \geq 16$.

the inlet mass flow remains constant to within $\pm 0.12 \%$ in the $S P$ case, and $\pm 0.09 \%$ in the $M P$ case. The turbine operating point may be taken as approximately constant after $f t \geq 16$.

\subsubsection{Rotor film cooling}

A fine time step, $\Delta t V_{\infty} / D \leq 0.02$, is required to resolve short length and time scales associated with rotor film cooling. At the cooling hole scale, both deterministic vane passing and non-deterministic turbulent unsteadiness are present. Convergence is reached when the flow is statistically stationary, i.e. at a particular vane phase, the ensemble average of a flow quantity is constant. On physical grounds, statistical stationarity may be assumed after one flow-through time, because film cooling performance is determined by a downstream convection process. In the present case, using rotor chord and a typical axial velocity, the flow-through time evaluates to 1.5 vane passing periods. Conservatively, two vane passing periods are allowed to propagate the start-up transient associated with refining the time step at $f t=16$, and instantaneous data are output for $f t \geq 18$ for further processing. 


\subsubsection{Unsteady data processing}

Ensemble averaging phase-locked to the vane passing extracts deterministic low-frequency unsteadiness associated with blade row interaction from a time series, and removes the non-deterministic turbulent unsteadiness. Here, a binning algorithm is used where the time series is divided into segments of length $\Delta f t=1 / 32$, which are then stacked into one of 32 bins each at constant vane phase. Taking the mean of all instantaneous data in a bin yields an ensemble average (assuming any variation is linear over the bin width). The root-mean-square deviation of the instantaneous data from the bin average then gives a measure of the non-deterministic unsteadiness component, or ensemble-averaged turbulence intensity. To make up a sector spanning one vane pitch, there are two identical rotor blades in the present computational domain. Data from both blades are used in the ensemble averaging, with a shift of 0.5 vane phase to account for their pitchwise offset. Collecting instantaneous data for $18 \leq f t \leq 22.5$ then allows averaging over 8 ensembles.

Illustrative ensemble data for momentum flux ratio and laterally-averaged film effectiveness are shown in Figures 8.3 and 8.4 respectively. The momentum flux ratio ensembles are consistent in shape, being set by the one-dimensional boundary conditions across the hole. However, for the leading-edge showerhead hole at $\widehat{f t}=0.25$, Figure 8.3(a), random unsteadiness is of similar order to the ensemble-averaged value because of the effect of large turbulent structures from the vane wake impinging on the blade leading edge.

Film effectiveness is determined by a turbulent mixing process, so exhibits higher levels of random unsteadiness than momentum flux ratio. Downstream of the front pressure-side hole SH1, at $\zeta / c_{x}=-0.32$ in Figure 8.4(a), deterministic unsteadiness accounts for only $10 \%$ of the instantaneous variance. Downstream of the suction-side hole SS, at $\zeta / c_{x}=0.68$ in Figure 8.4(b), deterministic unsteadiness accounts for $80 \%$ of the instantaneous variance. At this surface location, a $50 \%$ drop in film effectiveness during the vane passing cycle is resolved in the ensemble average, from $\overline{\varepsilon_{\mathrm{f}}} \approx 0.3$ for $0 \leq \widehat{f t} \leq 0.3$ to $\overline{\varepsilon_{\mathrm{f}}} \approx 0.15$ at $\widehat{f t} \approx 0.5$. As discussed below, this is attributed to periodic wake interaction. 

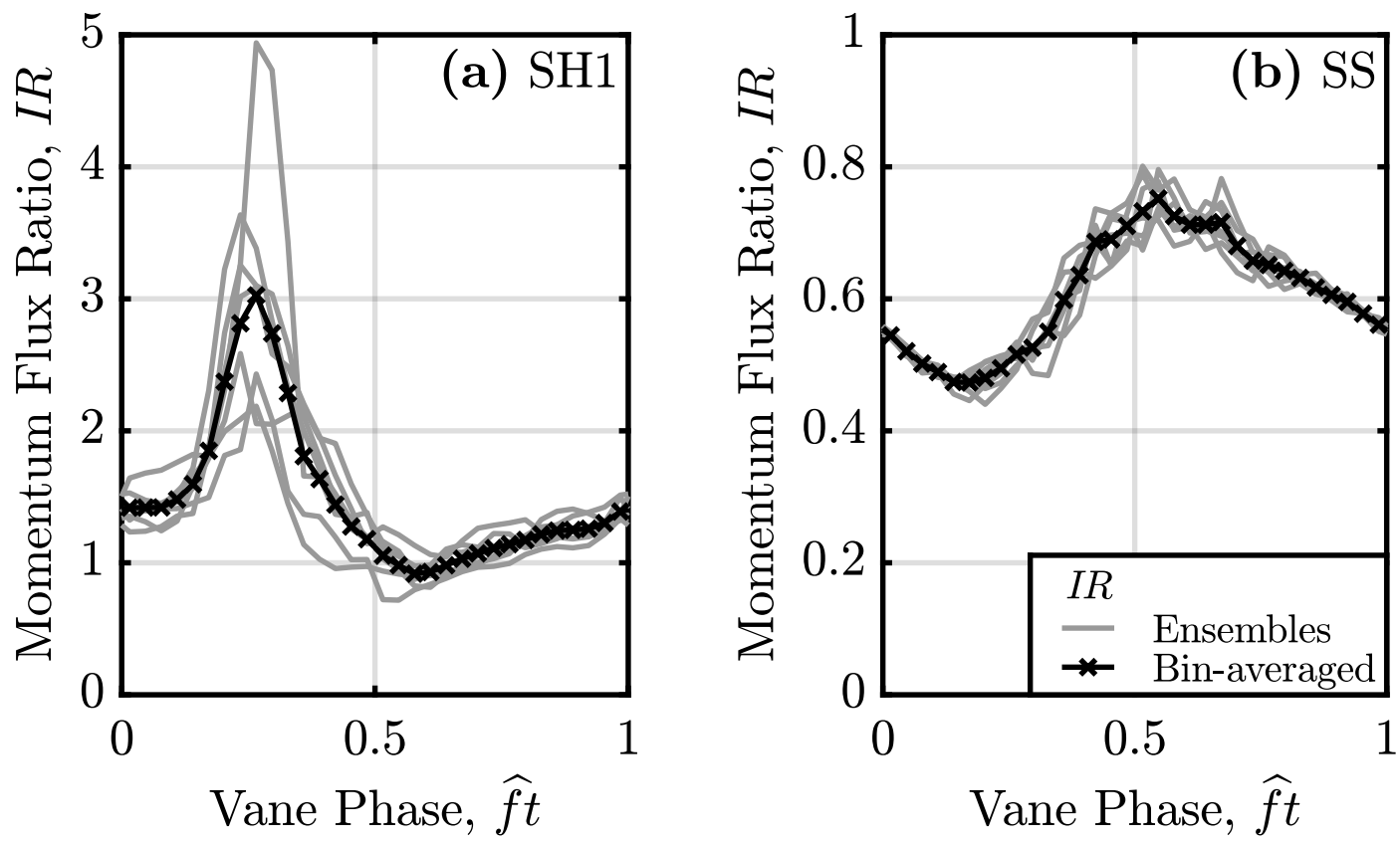

Fig. 8.3 Ensemble averaging of unsteady rotor cooling hole momentum flux ratio, (a) leading-edge showerhead hole SH1, (b) suction side hole SS. Non-deterministic unsteadiness is greater for the showerhead hole than the suction side hole.
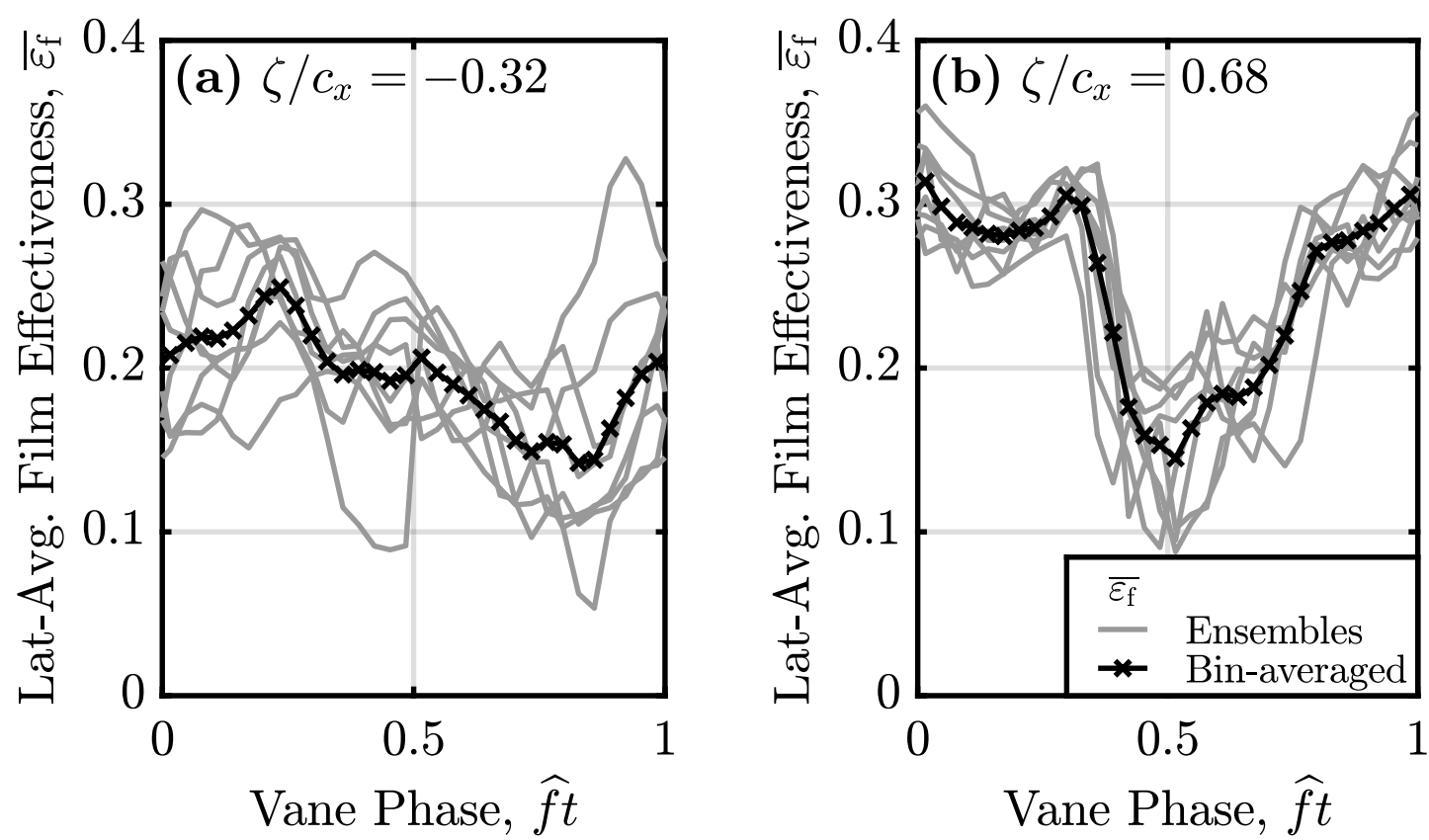

Fig. 8.4 Ensemble averaging of unsteady laterally-averaged rotor film effectiveness, (a) front pressure side, $\zeta / c_{x}=-0.32$, (b) downstream of suction-side hole, $\zeta / c_{x}=0.68$. 


\subsubsection{Boundary layers}

Resolution of the near-wall flow in the present simulations is confirmed by calculating the computational mesh cell sizes in wall units and comparing to known non-dimensional requirements. Figure 8.5 shows the distributions of near-wall cell sizes over the vane and blade surfaces.

On the vane surface, Figure 8.5(a), a URANS-only approach is used with a fullyturbulent wall function requiring a wall-normal cell size within the universal log-law region, $30 \leq \Delta_{\text {nor }}^{+} \leq 200$. This is achieved over $82 \%$ of the vane surface. Cooling patches at various locations on the vane increase local density and cause drops in $\Delta_{\text {nor }}^{+}$.

On the blade surface, Figure 8.5(b), data are shown for the streamwise, wall-normal, and lateral directions. Resolving the outer region of the boundary layer and film cooling requires a fine grid in all three directions. Target resolutions of $\Delta_{\mathrm{str}}^{+}=50$ in the streamwise direction, $\Delta_{\text {norm }}^{+}=1$ in the wall-normal direction, and $\Delta_{\text {lat }}^{+}=25$ in the lateral direction are chosen, based on the validated flat plate simulations in Chapter 7. The wall-normal spacing is $\Delta_{\text {norm }}^{+} \leq 1.4$ over the entire rotor blade surface, within $40 \%$ of the target and well within the validity of the laminar wall function of $\Delta_{\text {norm }}^{+} \leq 5$. The streamwise grid spacing is close to the target for 20 hole diameters downstream of injection locations, with $\Delta_{\text {str }}^{+} \leq 64$ and a maximum deviation of $28 \%$ from the target. The mesh is coarsened in the streamwise direction after 20 hole diameters downstream from cooling holes to reduce computational cost, with the grid spacing reaching up to $\Delta_{\text {str }}^{+}=300$. The lateral grid spacing is also on target in the near-hole regions, with $\Delta_{\text {lat }}^{+} \leq 33$ and a maximum deviation of $32 \%$ from the target, but rises downstream of $\zeta / c_{x} \geq 0.7$ up to $\Delta_{\text {lat }}^{+}=42$.

The resolution of the rotor film cooling is equivalent to that of the validated flat plate simulations up to 20 hole diameters downstream of injection locations. Given that the coolant film will be well-mixed by this point, and the large computational costs involved in the present simulations, coarsening of the mesh downstream is an acceptable compromise for the purposes of this investigation. 

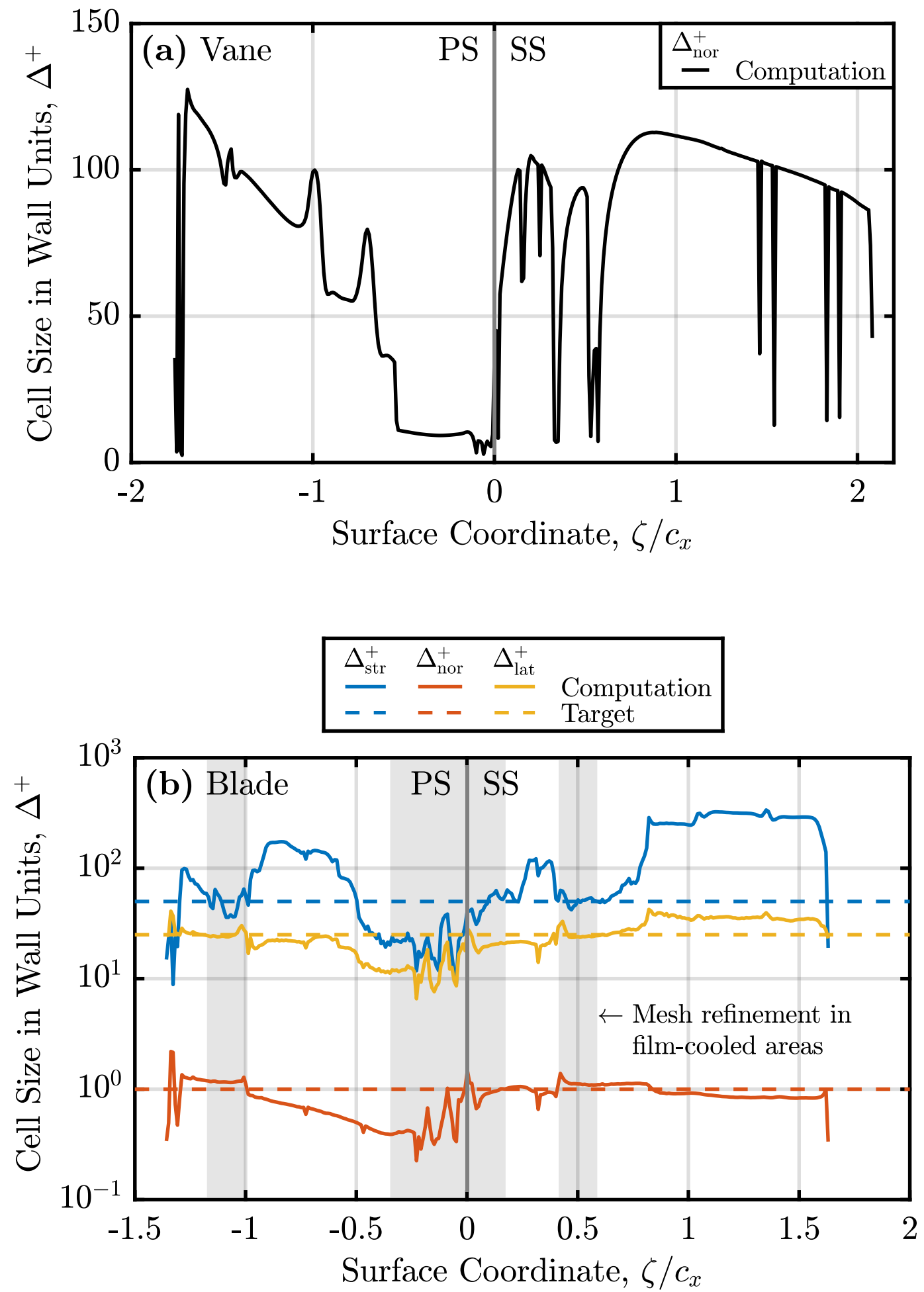

Fig. 8.5 Non-dimensional cell sizes in wall units for cascade: (a) vane, targeting log-law wall functions, (b) blade, target resolution based on flat plate simulations. 


\subsubsection{Vortical structure}

Figure 8.6 displays a snapshot of the instantaneous lateral vorticity field on the hole centre plane (cascade mid-span). The size of the vortical structures gives a qualitative picture of the spatial resolution achieved. There is no inlet turbulence, so, upstream of the vane trailing edge, vorticity is confined to the vane boundary layers, Figure 8.6(a), positive (blue) on the suction side and negative (red) on the pressure side. The vane boundary layers are modelled using URANS alone, and hence show no resolved turbulence. At the vane trailing edge, where the mesh is refined and the turbulent viscosity reduced to zero, the flow immediately transitions to resolved turbulence, Figure 8.6(b). The vortical structures associated with the cooling holes are smaller than those of the wake, Figure 8.6(c); the mesh is refined to the resolution of the flat plate simulations from Chapter 7 in this region. As the vane wake convects through the rotor throat, vorticity levels rise due to vortex stretching, and the fine structure of the wake is retained, Figure 8.6(d). 


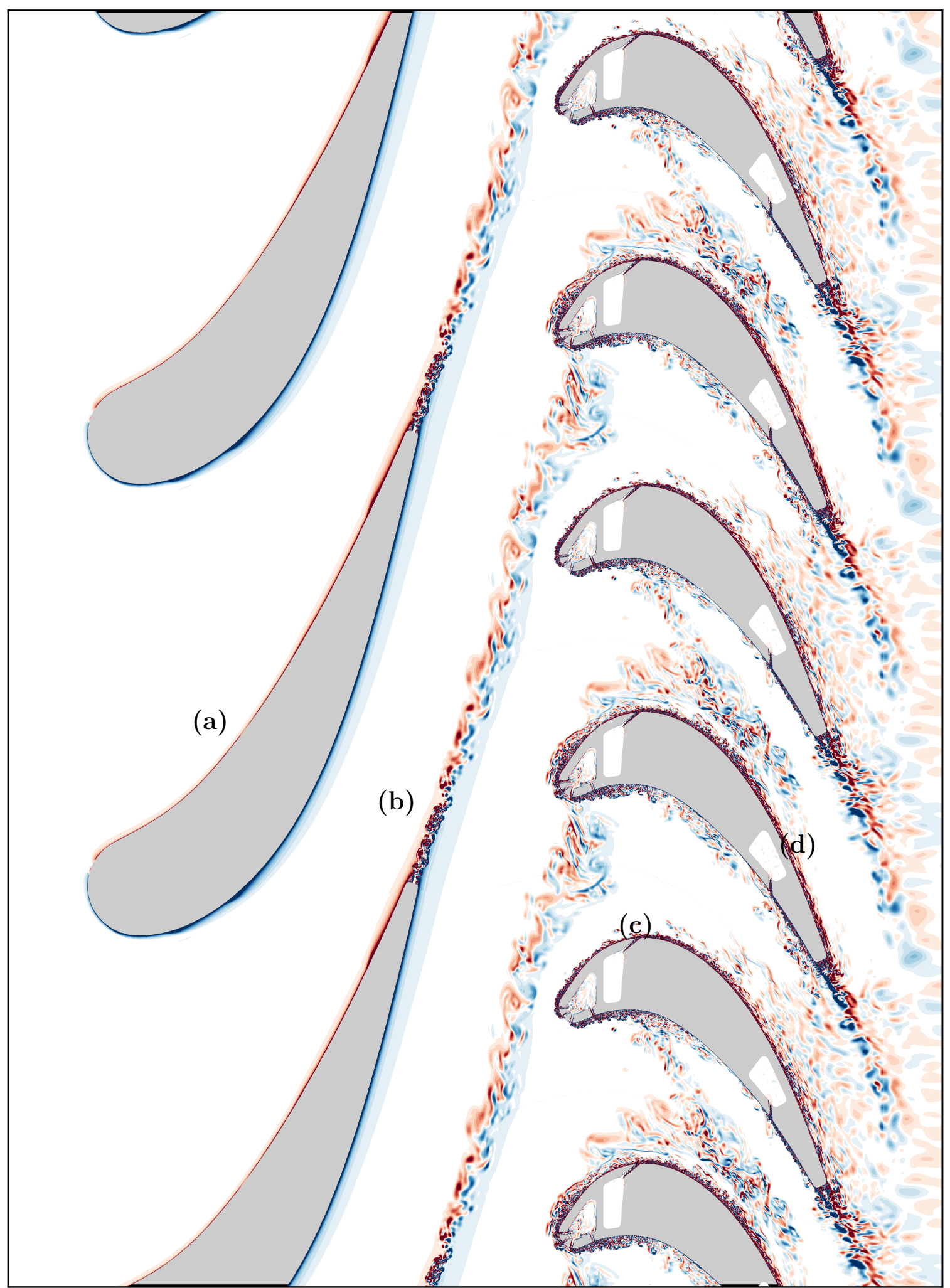

Fig. 8.6 Instantaneous lateral vorticity at cascade mid-span: (a) URANS vane boundary layers, (b) resolved turbulence in LES vane wake, (c) small-scale vortices in resolved rotor film cooling, (d) convecting vane wake retains structure. 


\subsection{Time-averaged flow field}

In this section, the time-averaged flow fields of the mixing-plane, $M P$, and sliding-plane, $S P$, cases are compared to each other and to a reference three-dimensional case.

\subsubsection{Comparison to three-dimensional case}

The cascade geometry is based on the mid-span section of the first high-pressure turbine stage of a large industrial gas turbine. Although a full three-dimensional geometry would be more representative, applying the hybrid URANS-LES method to such a case would be prohibitive in computational expense. Furthermore, using a linear cascade geometry isolates the blade row interaction mechanisms from confounding effects of secondary flows and three-dimensional blade design. Notwithstanding this simplification, the interaction mechanisms will be representative of the full three-dimensional turbine.

In Figure 8.7, vane wake profiles from the film-resolved $S P$ cascade computations are compared to results from the three-dimensional non-film-resolved simulation of the same stage presented in Chapter 6 . The profiles are extracted 0.22 axial chords downstream of the vane trailing edge. As well as confirming that the cascade flow is representative of the real turbine, good agreement would suggest that the hybrid URANS-LES is adequately resolving the vane wake.

The vane wake Mach number profile in the cascade is offset by 0.01 to 0.02 above the three-dimensional URANS result in Figure 8.7(a), however, the hybrid URANS-LES yields a similar shape. The peak-to-trough wake depths agree to within $6 \%$, and the wake widths agree to within 3\%. The offset is due to an increase in flow angle of $0.3^{\circ}$ in the cascade case, which is attributed to a change in vane loading due to a three-dimensional relief effect not present in the cascade. This is consistent with a $12 \%$ increase in the vane potential field amplitude in the cascade.

The vane wake stagnation temperature profiles are compared in Figure 8.7(b), plotted as an effectiveness referenced to vane (not rotor) inlet coolant conditions. The cascade URANS-LES peak wake effectiveness matches the three-dimensional URANS value to within $9 \%$, and the wake widths match to within $16 \%$. The integrated mass-averaged effectivenesses are 0.052 and 0.042 in the cascade and three-dimensional cases respectively, showing that the vane coolant mass flow per unit span is matched to within $24 \%$. 

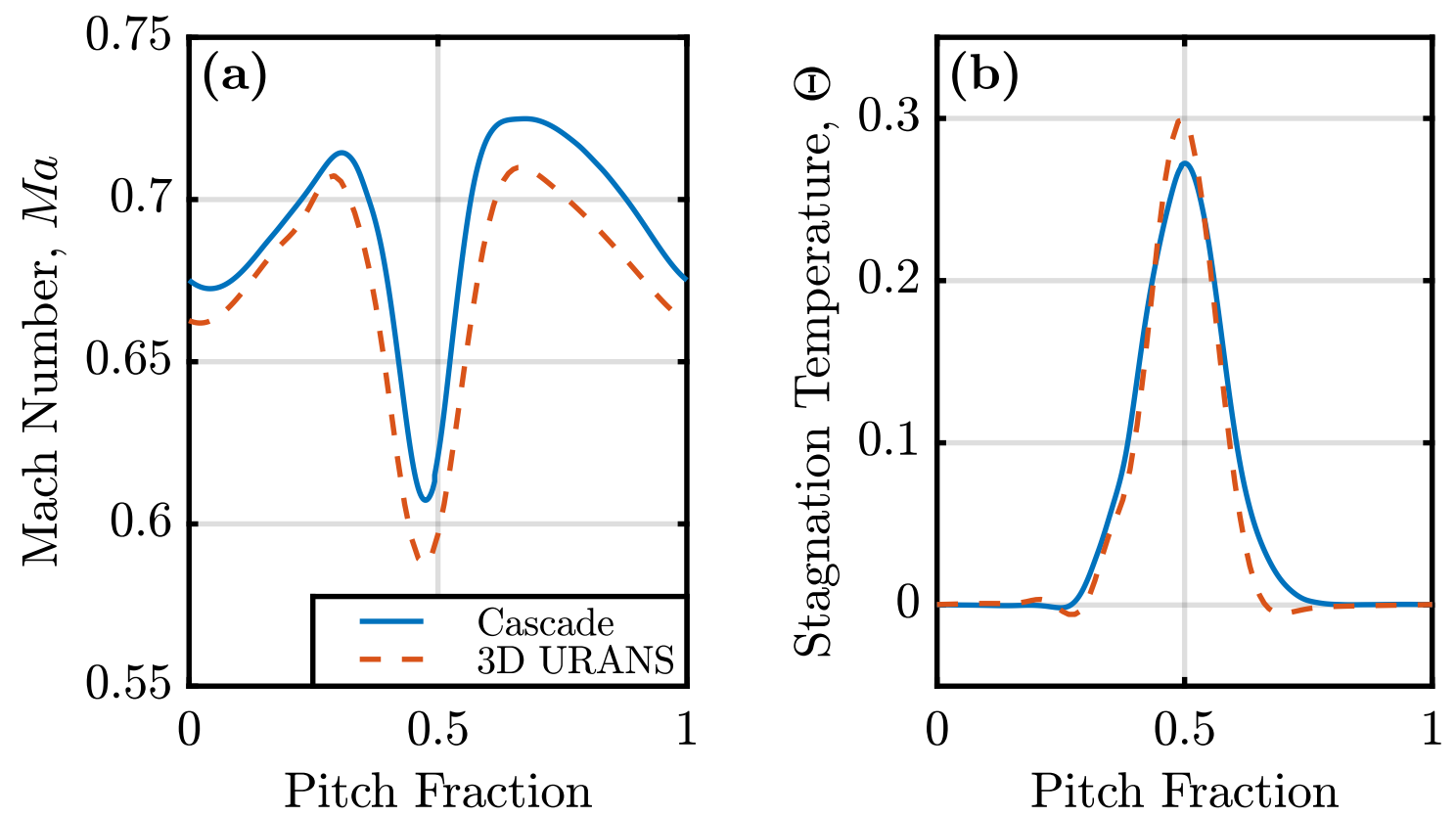

Fig. 8.7 Comparison of cascade hybrid URANS-LES and mid-span 3D URANS vane wake profiles: (a) Mach number, (b) stagnation temperature. The cascade wake is representative of the fully three-dimensional case.

\subsubsection{Effect of blade row interaction on film effectiveness}

For a turbine designer, the key quantitative metric for assessing cooling performance is the laterally-averaged film effectiveness. Because the time scale associated with conduction within the solid is slow compared with the vane passing period, the blade metal temperature responds to the time-averaged effectiveness only. A comparison of this parameter for the $M P$ and $S P$ simulations is displayed in Figure 8.8. The shaded bands denote uncertainty intervals to $95 \%$ confidence due to finite-time averaging. In this Chapter, film effectiveness is defined as,

$$
\varepsilon_{\mathrm{f}}=\frac{T_{\mathrm{aw}}-T_{0 \infty}^{\mathrm{rel}}}{T_{0 \mathrm{c}}^{\mathrm{rel}}-T_{0 \infty}^{\mathrm{rel}}}
$$

where $T_{\mathrm{aw}}$ is the local adiabatic wall temperature, $T_{0 \mathrm{c}}^{\mathrm{rel}}$ the rotor-relative blade coolant stagnation temperature, and $T_{0 \infty}^{\text {rel }}$ the reference rotor-relative main-stream temperature mass averaged at rotor inlet.

Film effectiveness is low at the blade leading edge, $\zeta / c_{x} \approx 0$. This is because the main-stream flow has low velocity, and hence the momentum flux ratio of the showerhead 


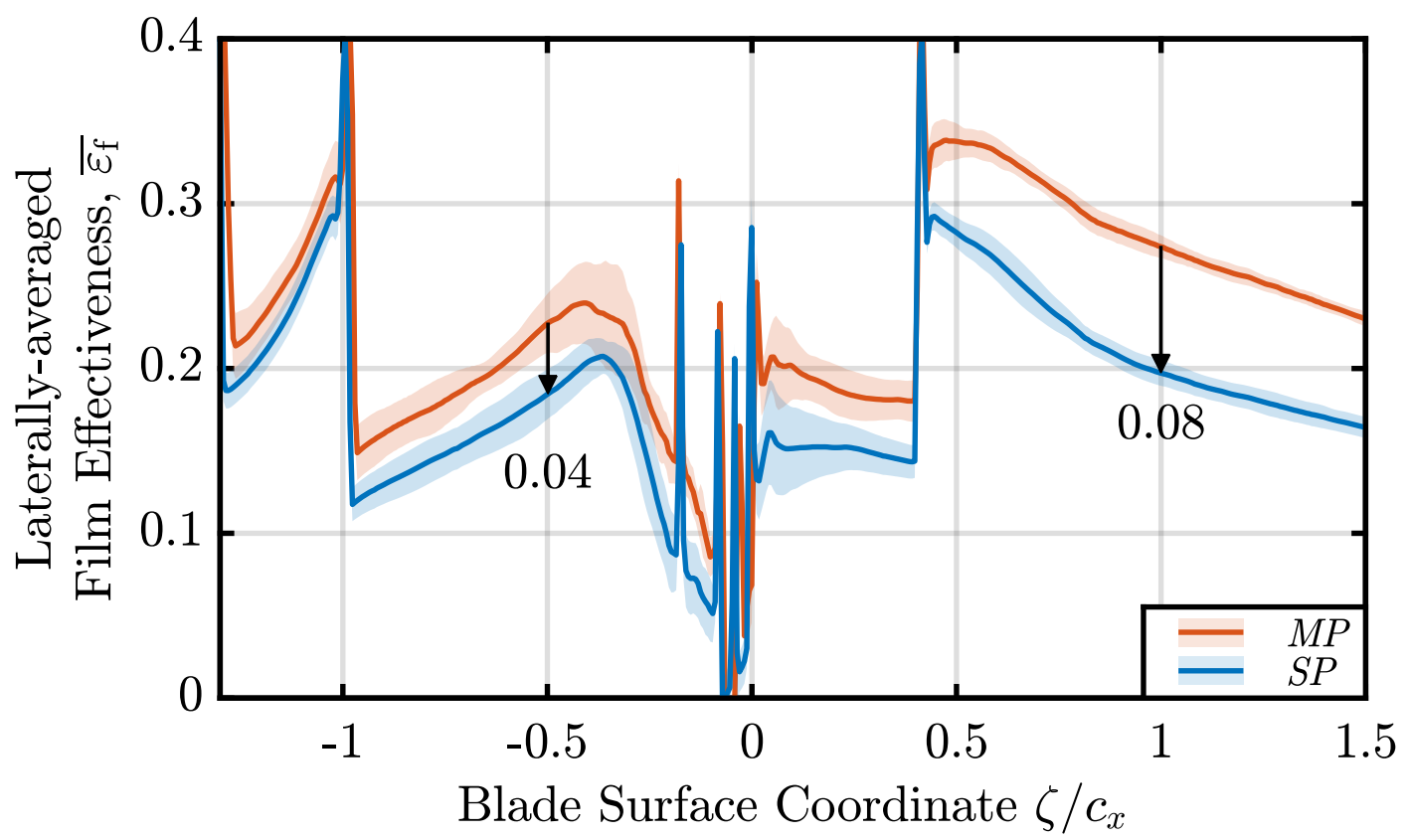

Fig. 8.8 Effect of blade row interaction on laterally-averaged film effectiveness, comparison of $M P$ and $S P$ computations. Effectiveness is reduced in the sliding plane case by up to 0.08 on the suction surface, $\zeta / c_{x}=0.9$.

cooling holes is high and the coolant is fully lifted-off from the blade surface. Typically, on a production blade, the showerhead holes have a compound angle to mitigate this effect. Also, supplementary to external film cooling, internal cooling within the holes and in the plenum using an impingement plate are used to control the leading-edge metal temperature.

Blade row interaction acts to reduce film effectiveness at all surface locations in the $S P$ case, by 0.02 to 0.04 or up to $18 \%$ on the pressure side, and 0.03 to 0.08 or up to $30 \%$ on the suction side. A rough estimate of the associated change in metal temperature, the final objective of cooling design, may be found using the method of Rutledge et al. (2016). Ignoring any possible increase in heat transfer coefficient from wake interactions (a conservative assumption), the method predicts a metal temperature rise of $10 \mathrm{~K}$ to $35 \mathrm{~K}$ due to blade row interaction. This is significant, given that a change in metal temperature of $25 \mathrm{~K}$ can reduce part life by a factor of two (Bogard and Thole, 2006).

At the scale of the rotor passage, the flow is behaving non-linearly, because the timeaveraged boundary conditions of the $S P$ and $M P$ cases are held constant. The remainder of this Chapter discusses the physical mechanisms that give rise to the predicted change in time-averaged film effectiveness. 


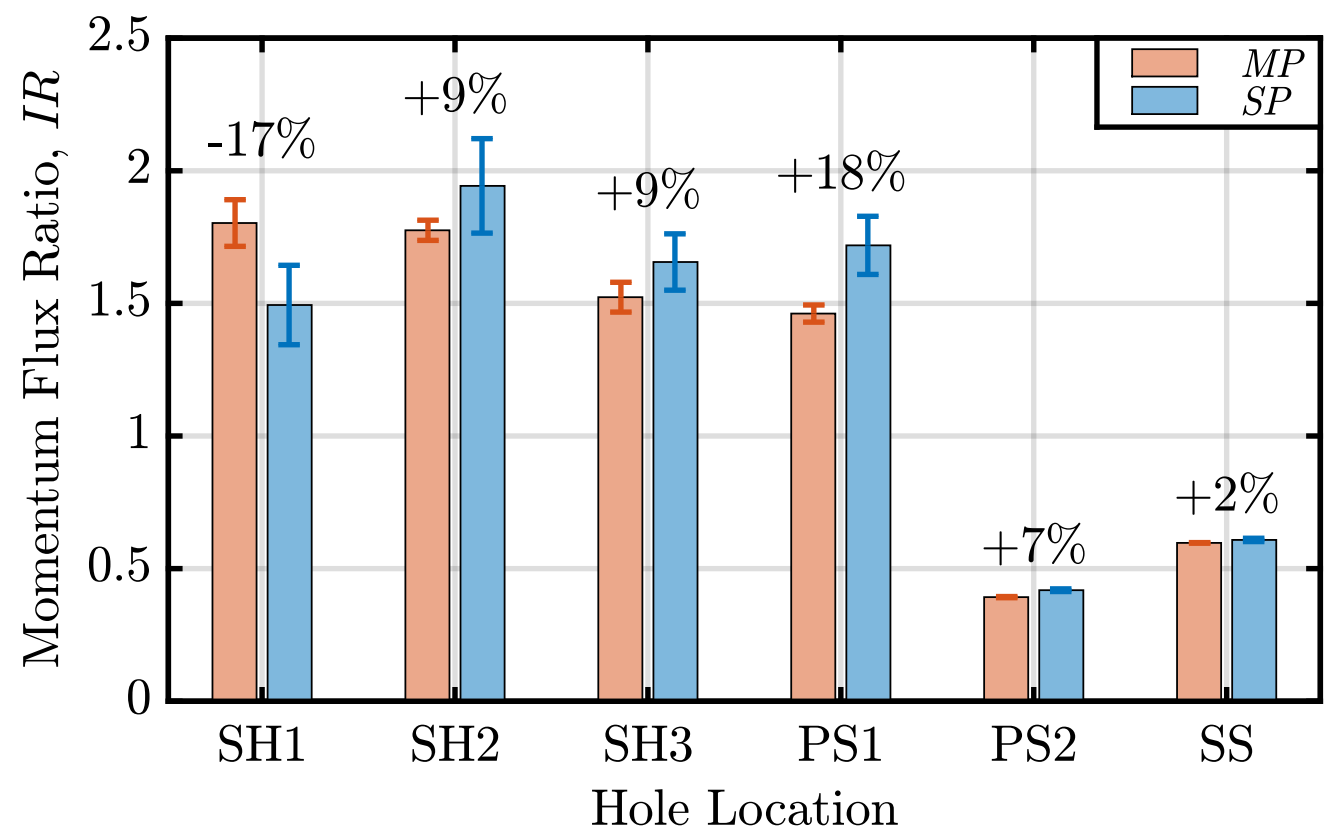

Fig. 8.9 Time-averaged momentum flux ratios for $M P$ and $S P$ computations, error-bars show $95 \%$ confidence interval due to finite-time averaging, annotations show $S P$ change relative to $M P$ case. Leading-edge holes match to within $\pm 18 \%$, and the pressure- and suction-side holes match to within $\pm 7 \%$ and $\pm 2 \%$ respectively.

\subsubsection{Cooling hole operating points}

If blade row interaction alters the time-averaged cooling hole operating point, then the time-averaged cooling performance may also change. Change in time-averaged momentum flux ratio is caused by both, changes in time-averaged main-stream boundary conditions, and non-linearity of the hole mass flow response, as demonstrated in Section 6.4.2.

The change in operating point due to blade row interaction is quantified in Figure 8.9, showing time-averaged momentum flux ratios for each cooling hole in both the MP and $S P$ computations. Error bars denote the uncertainty interval due to finite-time averaging of an unsteady flow, and the percent change in the $S P$ case with respect to the $M P$ case is labelled.

The momentum flux ratios of the showerhead cooling holes SH2 and SH3 agree to within the averaging uncertainty, while the momentum flux ratios for holes SH1 and PS1 are altered by blade row interaction by $\pm 18 \%$. These holes take fully-separated momentum flux ratios, at least $80 \%$ above the $I R \geq 0.8$ threshold for complete separation suggested by Bogard and Thole (2006), so are expected to be operating on a flat region of their performance characteristic. For $30^{\circ}$ inclined cylindrical holes studied in Chapter 5 , 
film effectiveness is constant to within the measurement uncertainty when increasing the momentum flux ratio from $I R=1.5$ to $I R=2.0$. The momentum flux ratios for hole SS agree to within $2 \%$, which would produce a negligible change in suction-side film effectiveness. For the rear pressure-side hole PS2, blade row interaction increases the time-averaged momentum flux ratio by $7 \%$. The hole, with $I R \approx 0.4$, is not operating on a flat region of the effectiveness characteristic. The flat plate data in Chapter 5 suggest a $7 \%$ increase in momentum flux ratio would result in a drop in time-averaged film effectiveness of order 0.005 .

Change in the time-averaged hole operating points will have a relatively small influence on the film effectiveness distribution, compared to other blade row interaction effects, and cannot account for the discrepancies in Figure 8.8.

\subsubsection{Vane coolant migration}

As identified in Section 6.3.1, with a cooled upstream vane, wake interaction causes vane coolant to migrate towards the rotor suction side. Compared to a case where the wake is mixed out to uniformity, the temperature on the pressure side of the rotor passage is thus increased. The local rotor main-stream temperature is altered, changing the adiabatic wall temperature and hence apparent cooling performance, without implying any change in film behaviour.

The effect is quantified in Figure 8.10, which shows distributions of time-averaged stagnation temperature throughout the rotor passage with and without blade row interaction. The temperature is plotted as an effectiveness $\Theta$, defined in Equation (6.3), such that $\Theta=0$ corresponds to the mixed-out rotor inlet temperature, and $\Theta=1$ corresponds to full cooling on the blade surface.

In the $M P$ case, Figure 8.10(a), the main-stream temperature $\Theta \approx 0$ throughout the rotor passage as expected from the lack of wake interaction. In the $S P$ case, Figure 8.10(b), effectiveness is negative towards the pressure side, and positive towards the suction side of the passage. The pattern observed is the same as in the non-film-resolved computations of Chapter 6. A dotted line contour shows an approximate boundary of the coolant film based on the $M P$ temperature field; the main-stream location may be taken as just outside this region.

For the $S P$ case, Figure 8.10(b), towards the pressure side of the rotor passage, the main-stream stagnation effectiveness is between 0.025 and 0.045 . Differences between the two rotor blades are due to finite-time averaging. It can be shown that the change in film effectiveness, due to a small modification in main-stream temperature $\Theta \ll 1$, is to first order $\Theta\left(1-\varepsilon_{\mathrm{f}}\right)$. The drop in pressure-side effectiveness of between 0.02 
(a) $M P$

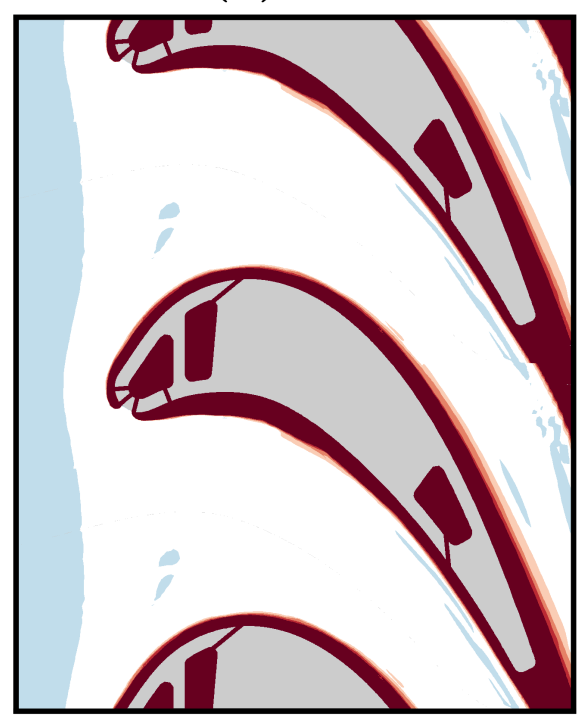

(b) $S P$

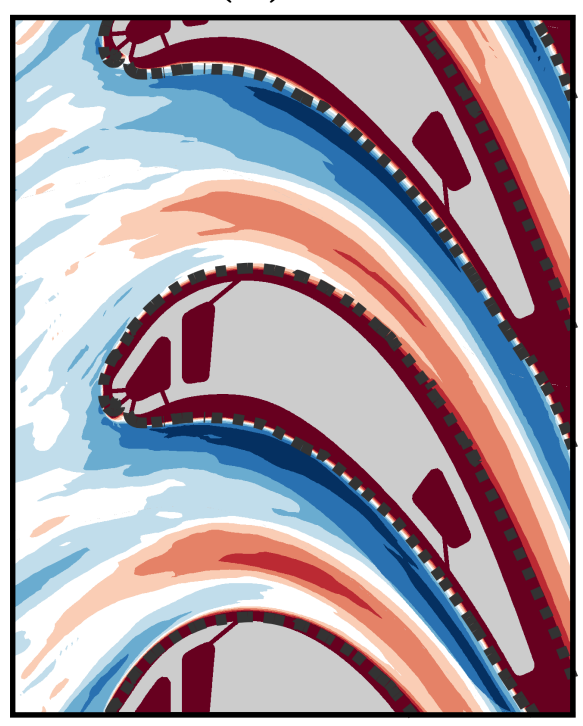

0.04

(1)

0.02

Fig. 8.10 Time-averaged stagnation temperature at cascade mid-span, (a) $M P$ computation, (b) $S P$ computation. Migration of vane coolant alters the temperature field by \pm 0.04 in film effectiveness.

and 0.04 in Figure 8.8 for $\zeta / c_{x}<-0.2$ is therefore quantitatively consistent with the change in main-stream temperature due to vane coolant migration. Considering the suction side, main-stream effectiveness is between -0.01 and 0.025 , and less than 0.01 for approximately the first half of surface length. The drop in effectiveness of up to 0.08 in Figure 8.8 for $\zeta / c_{x}>0.1$ is not explained by vane coolant migration.

\subsection{Unsteady flow field}

\subsubsection{Main-stream turbulence intensity}

In the $M P$ computation, the vane wake is mixed out before entering the rotor, giving a uniform inflow and no inlet turbulence. In the $S P$ computation, the wake convects into the rotor passage and periodically increases the turbulence level. This means that, regardless of any unsteady non-linear effects, the time-averaged turbulence intensity has changed, which is expected to alter cooling performance.

In a case with periodic unsteadiness, as in the $S P$ case here, the time-averaged turbulence intensity should be calculated by taking the mean ensemble-averaged turbulence intensity over the complete vane passing. This ensures that the value of $T u_{\infty}$ characterises 


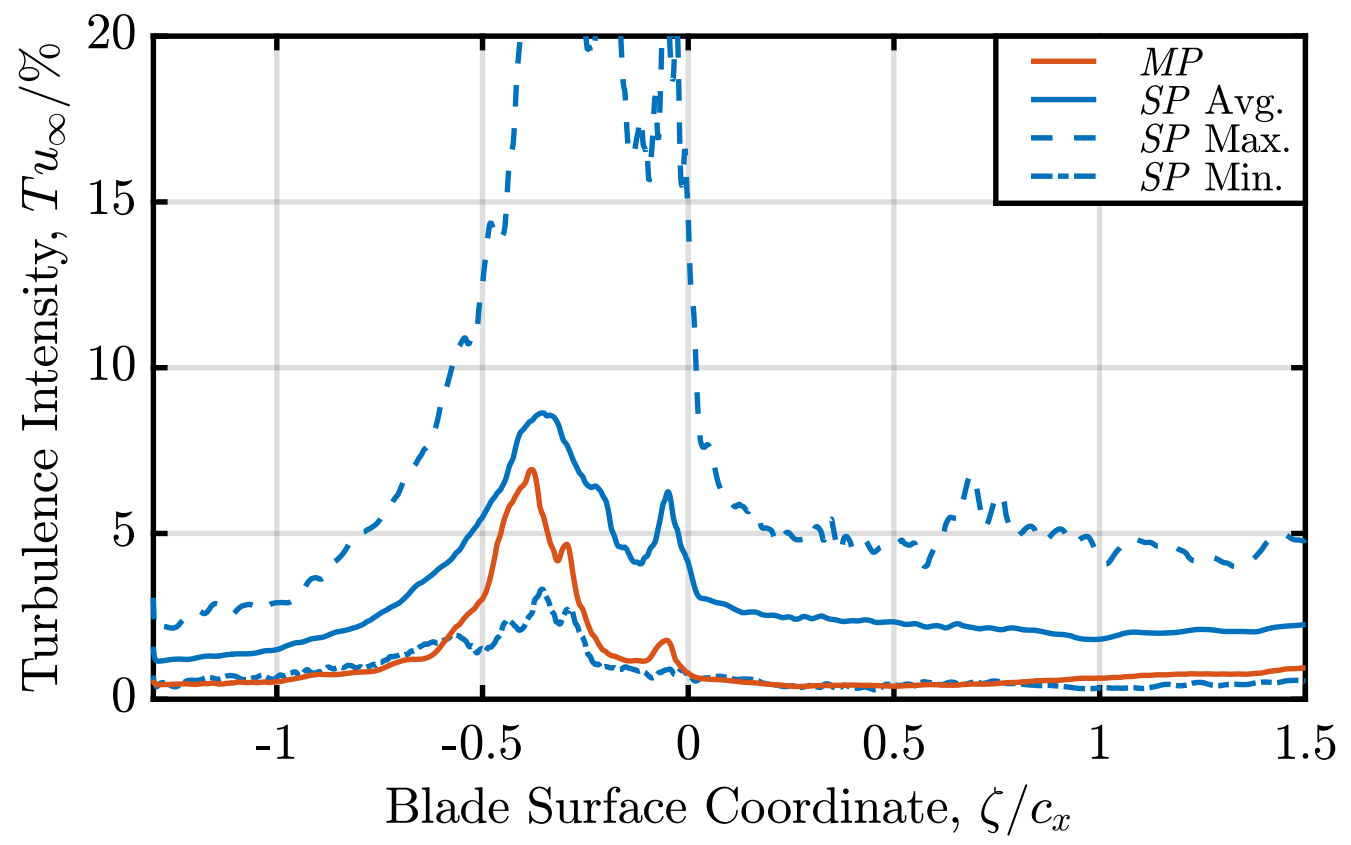

Fig. 8.11 Main-stream turbulence intensity over rotor blade surface. Blade row interaction increases the maximum turbulence intensity, but the minimum remains the same.

non-deterministic effects alone, rather than including periodic velocity fluctuations that are not turbulent in origin.

Figure 8.11 shows the variation in main-stream turbulence intensity over the rotor blade surface for the two computational cases. The $M P$ case is characterised by a single line corresponding to the 'steady' or statistically-stationary turbulence intensity. Three lines are shown for the $S P$ case: the minimum and maximum ensemble-averaged turbulence intensity, and the time-averaged turbulence intensity. The results are calculated from velocity data on the hole centreline at a constant offset of five hole diameters from the rotor blade surface. This distance was fixed during the meshing process, based on an estimated film penetration depth. A consequence of this is that the data for $-0.7 \leq \zeta / c_{x} \leq 0$ are not a true main-stream turbulence intensity, as the offset is not far enough to avoid contamination from turbulence generated by the film cooling itself.

Outside of $-0.7 \leq \zeta / c_{x} \leq 0$, the $M P$ turbulence intensity is low, $T u_{\infty} \leq 1 \%$, and thus is expected to have a negligible effect on film effectiveness. The corresponding time-averaged $S P$ turbulence intensity is greater as expected, between $2 \% \leq T u_{\infty} \leq 3 \%$ on the suction side, and $1 \% \leq T u_{\infty} \leq 2 \%$ on the pressure side. However this masks variations during the vane passing: turbulence intensity rises to a maximum of $T u_{\infty} \approx 5 \%$ 
on the suction side, while the minimum is within $0.5 \%$ of the $M P$ values. This shows that, after the wake has passed a surface location, the main-stream turbulence intensity returns to the negligible value that would be expected without blade row interaction.

Near the leading edge, for $-0.5 \leq \zeta / c_{x} \leq-0.2$, the $S P$ maximum turbulence intensity rises to $T u_{\infty} \approx 20 \%$, due to direct impact of turbulent structures from the vane wake. Here, the $S P$ minimum turbulence intensity is lower than the $M P$ values. This is hypothesised to be caused by unsteady movement of the showerhead coolant trajectories closer to the blade surface.

\subsubsection{Ensemble-averaged film effectiveness}

The ensemble-averaged data resolve periodic variation in instantaneous cooling performance during a vane passing period. Although the metal temperature is set only by the time-averaged performance, the unsteady data permit a more detailed examination of the physical mechanisms responsible for the effect of blade row interaction on the time-averaged value.

Figure 8.12 shows distributions of laterally- and ensemble-averaged film effectiveness in the $S P$ case. The ensemble minimum distribution is found by taking the minimum effectiveness across all vane phases at each surface location, and the ensemble maximum is defined analogously. Also shown for reference are the time-averaged $S P$ and $M P$ distributions from Figure 8.8.

The peak-to-trough amplitude of unsteady excursions in effectiveness is greatest near the leading edge, reaching 0.3 downstream of the showerhead at $\zeta / c_{x}=-0.1$. Unsteadiness in this region is due to direct impingement of the vane wake, bringing vane coolant to the blade surface and modulating the showerhead coolant trajectory. Amplitudes of excursion are lower on the pressure side, less than 0.018 for $\zeta / c_{x} \leq-0.32$. The vane wake does not impinge on the pressure side, where unsteadiness is generated by a different mechanism. Isentropic compression and expansion due to potential field pressure waves, and instantaneous excursions in momentum flux ratio, are both plausible candidates. In the present case, these effects produce a linear response in film effectiveness, so that they do not contribute to the change in time-averaged performance for $\zeta / c_{x}<-0.2$ (which is due to vane coolant migration only).

The blade suction side, where $\zeta / c_{x} \geq 0.1$, exhibits first-order unsteadiness in film effectiveness, with a peak-to-trough amplitude of up to 0.19 or $90 \%$ of the time-averaged value. The $S P$ ensemble maximum is similar to the steady $M P$ distribution, matching to within \pm 0.025 , and to within \pm 0.005 for $\zeta / c_{x} \geq 0.7$. Despite first-order excursions in film effectiveness, the maximum cooling performance is comparable to that achieved 


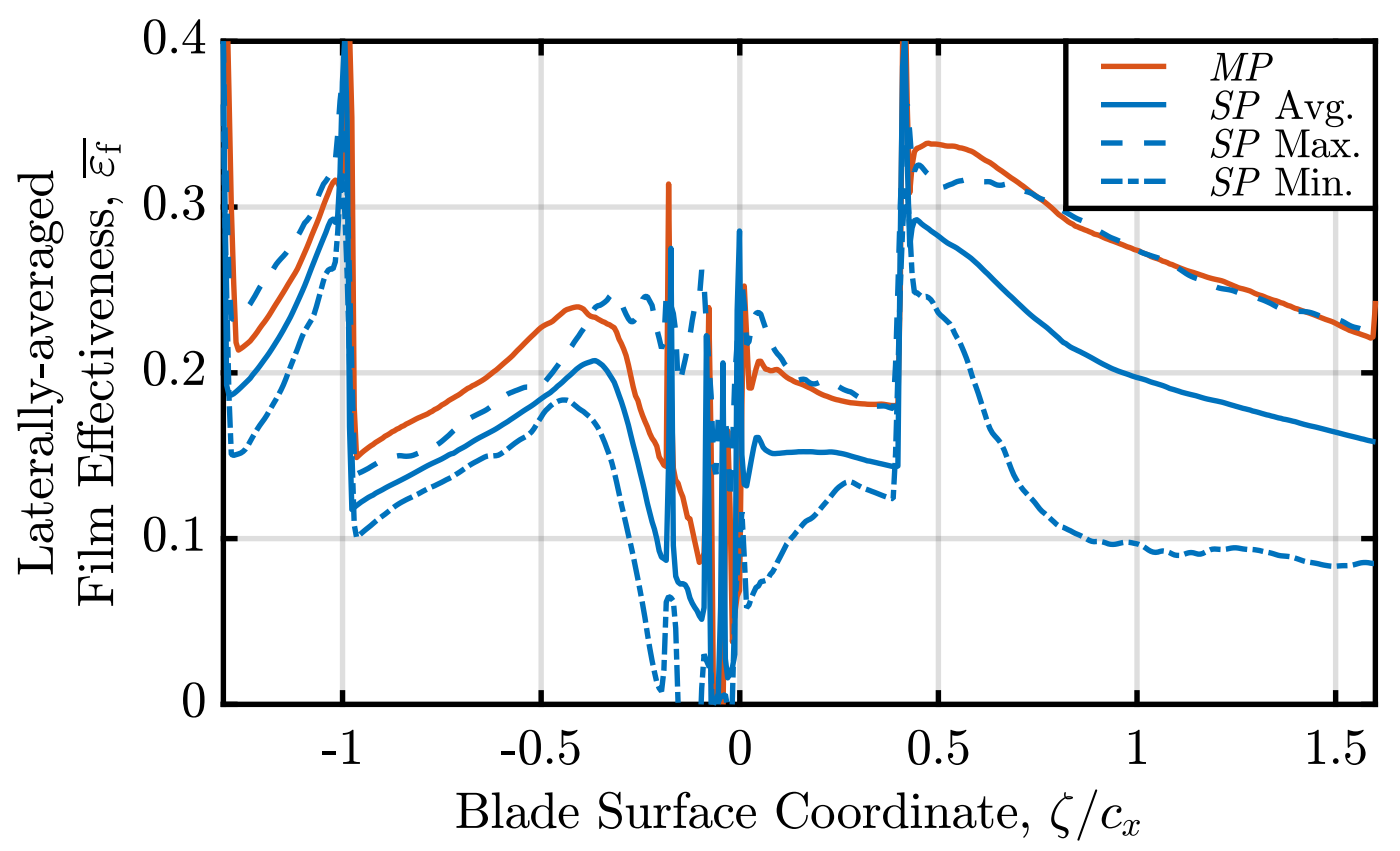

Fig. 8.12 Unsteady behaviour of rotor film effectiveness, comparison of $S P$ time-average, ensemble maximum and ensemble minimum laterally-averaged film effectiveness over rotor blade surface. On the suction side, the ensemble maximum effectiveness is similar to the $M P$ value, while a deficit remains on the pressure side. 
with steady inflow conditions. The $S P$ ensemble minimum shows a rapid decay with streamwise distance: the effectiveness gradient, $\mathrm{d} \overline{\varepsilon_{\mathrm{f}}} / \mathrm{d}(x / D)$, at $\zeta / c_{x}=0.6$ is 2.5 times steeper than the corresponding value from the $M P$ distribution, resulting in a reduction in film effectiveness of $61 \%$ at the trailing edge relative to the $M P$ value. The large range of excursions in suction-side film effectiveness are suggestive of non-linear behaviour, which is consistent with the drop in time-averaged film effectiveness at these locations.

Unsteady variation in film effectiveness on the suction side is due to periodic interactions as the wake passes over the film. At instants between wakes, the $S P$ ensemble minimum turbulence intensity, and ensemble maximum film effectiveness, both coincide with the $M P$ values. When a vane wake arrives, the main-stream turbulence intensity increases to $T u_{\infty} \approx 5 \%$ (Figure 8.11), and there is a kinematic perturbation to the velocity field of order $10 \%$ isentropic exit velocity (Figure 6.2). The net result is an increase in the rate of film mixing, which reduces instantaneous cooling performance. The magnitude of the effects that would be expected from both main-stream turbulence and velocity field perturbations will now be addressed in turn.

For cylindrical cooling holes on a flat plate, main-stream turbulence decreases film effectiveness when coolant is attached at low momentum flux ratio, and increases film effectiveness when coolant is separated from the wall at high momentum flux ratio. For example, Schmidt and Bogard (1996) reported a reduction of $53 \%$ at $I R=0.2$ due to main-stream turbulence with $T u_{\infty}=17 \%$, and an $86 \%$ increase at $I R=2.0$. Interpolating their measurements to the operating point of hole SS, $I R \approx 0.6$, a reduction of $15 \%$ is expected. The effect is smaller than the $60 \%$ reduction of the from ensemble maximum to minimum in Figure 8.8, despite the higher turbulence intensity of $T u_{\infty}=17 \%$ in the experiment compared to the ensemble maximum of $T u_{\infty} \approx 5 \%$ in Figure 8.11. In another study, Baldauf et al. (2002) reported a decrease in film effectiveness of up to $30 \%$ due to main-stream turbulence with $T u_{\infty}=4 \%$ at $I R=0.46$, closer to the present operating points.

In the literature, the magnitude of the main-stream turbulence effect is a strong function of momentum flux ratio and turbulence intensity. There are no reported studies for which a direct comparison to the present case can be made, but the detrimental effect of wake interaction in the cascade is larger than might be expected from only main-stream turbulence effects at the ensemble maximum $T u_{\infty} \approx 5 \%$.

However, in the present computation, wake-generated main-stream unsteadiness has a much larger length scale than used in flat-plate experiments, as evident from the vortical structure illustration in Figure 8.6. Quantitatively, integration of the longitudinal two-point spatial correlation yields a main-stream turbulent length scale $\Lambda / D=7.6$ on 
the rotor suction side at $\zeta / c_{x}=0.4$, whereas the largest length scale reported in the film cooling literature is $\Lambda / D=3$ by Schmidt and Bogard (1996). Furthermore, the present blade geometry is not a flat plate with zero pressure gradient as commonly tested. The suction surface is convex with a favourable pressure gradient, both of which are known to affect film cooling performance (Bogard and Thole, 2006). Both of these variables may lead to a greater effect of main-stream turbulence than estimated from flat-plate measurements above.

The second effect of wake interaction is a perturbation of the film cooling velocity field due to the negative jet impinging on the suction side. Moving-bar wake generators can be used to model the velocity perturbation in a flat plate test, but do not decouple it from an increase in main-stream turbulence. The studies reviewed in Section 2.4.2 find that bar wakes have an effect similar to that of a steady main-stream turbulence intensity on flat plates, increasing film effectiveness when coolant is separated. However, for example in the cascade configuration of (Du et al., 1999) at momentum flux ratios similar to the present case, bar wakes result in a reduction in film effectiveness of between $10 \%$ and $30 \%$ on both the pressure and suction sides. A closer approximation of a pure impingement velocity perturbation, without turbulence, is the study of Borup et al. (2018), where an unsteady main stream is generated by a pitching aerofoil, and the effect on film effectiveness is measured. The data show a $20 \%$ reduction in time-averaged film effectiveness near the hole, and a $20 \%$ increase 15 diameters downstream.

Although the character of the velocity field perturbation will be a strong function of the geometry, and the few data available for comparison do not closely match the present test conditions, it is plausible that the velocity field perturbation could account for the drop of up to $30 \%$ in time-averaged suction-side film effectiveness seen here. A definitive statement would require analysis of the fully-three-dimensional, ensembleaveraged flow fields, which have not been collected during the computation. This would allow separation of the deterministic velocity perturbation from stochastic turbulence effects on the behaviour of the coolant jet.

Further evidence that unsteadiness in film effectiveness on the suction side is due to wake interaction is shown in Figure 8.13. This is a space-time diagram of laterallyand ensemble-averaged film effectiveness over the rotor blade surface. The coloured contours show film effectiveness, and line contours constant turbulence intensity $T u_{\infty}=$ $1 \%$. For $\zeta / c_{x}>0$, the line contours indicate a low-turbulence region between wakes, convecting downstream and associated with high film effectiveness. Outside of these regions, instantaneous performance reduces. For example, at $\zeta / c_{x}=0.9$, effectiveness is 
Laterally-averaged Film Effectiveness, $\left\langle\overline{\varepsilon_{\mathrm{f}}}\right\rangle$
$T u_{\infty}=1 \%$
0.1
0.2
0.3

$-$
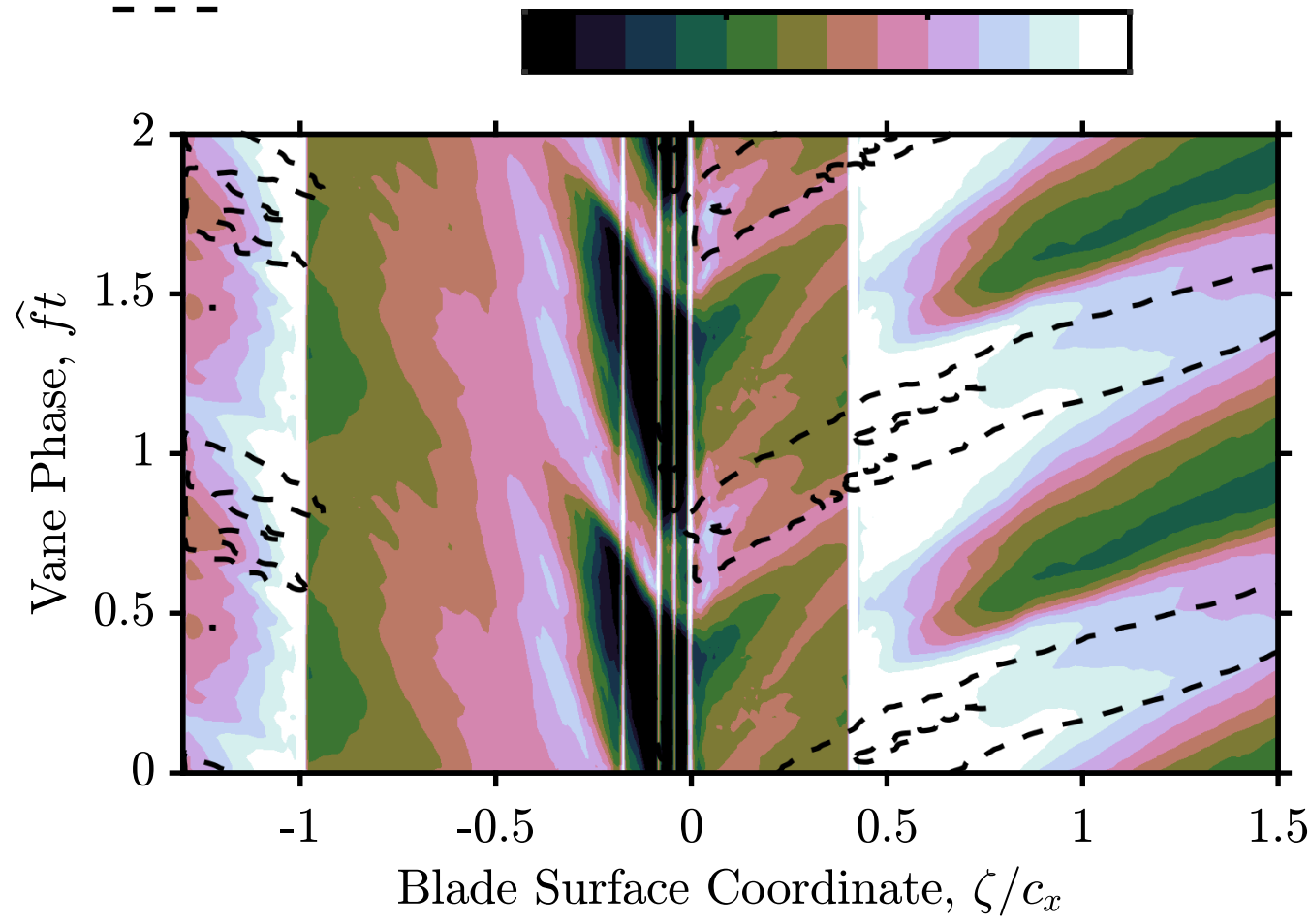

Fig. 8.13 Space-time diagram of laterally- and ensemble-averaged rotor film effectiveness, with line contours of constant main-stream turbulence intensity indicating the vane wake path. Arrival of the vane wake reduces instantaneous film effectiveness.

$\overline{\varepsilon_{\mathrm{f}}} \geq 0.25$ between wakes, but sharply reduces to $\overline{\varepsilon_{\mathrm{f}}}=0.1$ when a wake arrives at $\widehat{f t}=0.6$ before recovering during $0.6 \leq \widehat{f t} \leq 1$.

The unsteady range of film effectiveness, and maximum turbulence intensity, are lower on the pressure side than on the suction side. This is because the wake negative jet is directed away from the pressure side. The implication is that, when attempting to model blade row interactions interaction in a steady design framework, the main-stream turbulence intensity should be non-uniform. Put another way, the results of the present computations suggest that using a turbulence intensity measured upstream of the rotor as a main-stream boundary condition over both the rotor pressure and suction sides will lead to an under-estimation of cooling performance on the pressure side. 


\subsection{Assessment of quasi-steady framework}

The estimated reduced frequency of $\kappa_{\mathrm{c}} \approx 0.06$ from Section 2.4.1 suggests that the rotor cooling hole flow may be taken as quasi-steady subject to blade row interaction unsteadiness. Based on this assumption, a quasi-steady hole response model is developed in Chapter 6. In Chapter 5, it is concluded that, at a representative low reduced frequency, the effect of unsteadiness is set by non-linearity in the hole response. In this section, computational results from the current Chapter are interpreted in the framework of the earlier findings of this thesis.

\subsubsection{Validation of hole modelling}

Chapter 6 presents a reduced-order modelling approach to assess the effect of blade row interaction on rotor film cooling. Unsteady main-stream boundary conditions are taken from URANS CFD, and used as input to a hole response model to predict instantaneous excursions in cooling hole operating point. The key assumption is that the hole flow is quasi-steady, and that a discharge coefficient measured in steady flow can be applied instantaneously in the unsteady flow. This is justified based on the difference in characteristic time scales, i.e. low reduced frequency $\kappa_{\mathrm{c}} \approx 0.06$. The present resolved cooling hole computations with blade row interaction allow direct validation of this model.

First, discharge coefficients for each rotor cooling hole in steady conditions must be determined. These are calculated from the $M P$ computation, rather than experiments or correlations, to give a consistent comparison with the $S P$ computation. The discharge coefficients are assumed constant at the $M P$ values during unsteady excursions. Laterallyand ensemble-averaged main-stream boundary conditions are extracted from the $S P$ results and used as input to the same formulation of quasi-steady model as described in Chapter 6.

A comparison of the resolved ensemble-averaged momentum flux ratio, and that predicted by the model is shown in Figure 8.14. Normal-angled and inclined holes are displayed separately in Figures 8.14(a) and 8.14(b) respectively. The quasi-steady model is reassuringly accurate. For the holes not situated on the leading edge, PS1, PS2 and $\mathrm{SS}$, the root-mean-square error is less than 5\%. However, for the showerhead holes SH1, SH2, and SH3, there are discrepancies with the model predictions, and up to $18 \%$ root-mean-square errors. For example, with hole SH2, the model under-predicts the peak in momentum flux ratio at $\widehat{f t} \approx 0.3$ by $17 \%$, and over-predicts the trough at $\widehat{f t} \approx 0.7$ by $30 \%$. The former could be attributed to invalidity of the quasi-steady assumption due to 


\section{- SP resolved holes \\ - - Quasi-steady model}
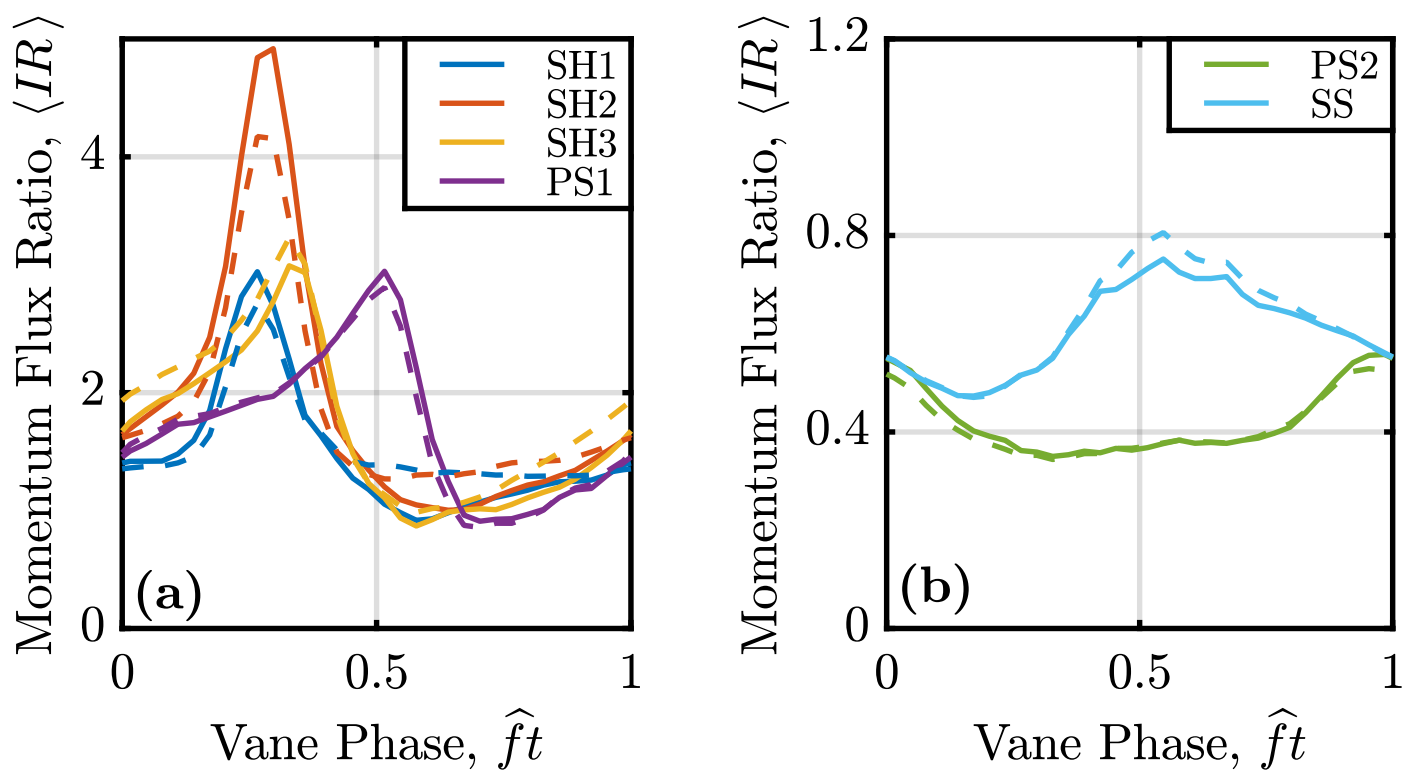

Fig. 8.14 Validation of quasi-steady hole response model by comparison with resolved SP ensemble-averaged momentum flux ratio, (a) normal holes, (b) inclined holes. Rootmean-square errors are less than $18 \%$ for holes SH1, SH2 and SH3 on the leading edge, and less than $5 \%$ for holes on the blade surface PS1, PS2 and SS.

a high temporal gradient in the flow, but this is not consistent with the latter, when the flow is varying more slowly. The discrepancy is instead attributed to the sensitivity of defining a single main-stream reference point in high spatial gradients around the leading edge. Overall, agreement between the resolved-hole computations and the quasi-steady model is good, supporting the quasi-steady assumption for the hole flow, and use of the hole response model. This analysis is concerned with the hole flow only: the proceeding section considers the more complex problem of predicting film effectiveness.

\subsubsection{Film effectiveness modelling}

It is less straightforward to assess the quasi-steadiness or non-linearity of film response in a realistic case such as the present cascade with wake interactions, compared to the idealised flat-plate experiments with potential field forcing in Chapter 5. For example, in Figure 5.6, film effectiveness is measured at a range of $I R$; it would be possible to simulate 
the cascade with a range of steady inlet turbulence intensities to check for non-linearity in that variable, but an analogous procedure to generate a response characteristic for negative jet velocity perturbations is intractable.

Wake passing creates a continuous variation in the main-stream velocity and temperature fields over the blade surface. In this situation, the entire history of perturbations upstream affect local film effectiveness, and so the definition of an equivalent global flow with steady boundary conditions for each instant during the wake passing is not possible. Without such an equivalent flow, the concept of quasi-steadiness is ambiguous. Modelling could proceed assuming that the local rate of mixing is quasi-steady, and an integration performed proceeding downstream from the hole exit using instantaneous main-stream conditions at every point. This would account for history effects within the quasi-steady framework, but the problem remains of defining an equivalent local steady flow for each instant during the wake passing.

On the scale of the rotor passage, the wake interactions are demonstrably non-linear, because the time-averaged boundary conditions at the rotor inlet exhibit negligible change between the mixing plane and sliding plane cases, but the time-averaged film effectiveness is significantly altered. The definition of a quasi-steady response characteristic is useful for modelling and illustration purposes, but is not required for the previous arguments on non-linearity to hold. 


\subsection{Conclusions}

In this Chapter, film-resolved computations of a turbine stage linear cascade are used to quantify the effect of blade row interaction on film cooling performance, and explain the physical mechanisms responsible. Two cases are simulated, with either a mixing or sliding plane at the rotor-stator interface. The following conclusions are drawn from the results:

1. Compared to a uniform, steady rotor inflow, blade row interaction reduces laterallyaveraged film effectiveness at all rotor surface locations. The suction side is most affected, with a reduction of between 0.03 and 0.08 , while the pressure side experiences a reduction of between 0.02 and 0.04 .

2. The effect of blade row interaction on film effectiveness cannot be attributed to changes in time-averaged cooling hole operating points. Momentum flux ratios of the four normal-angled holes near the leading edge change by up to $\pm 18 \%$, but are all fully lifted-off, and operating on a flat region of their film effectiveness characteristic. Momentum flux ratios of the inclined pressure- and suction-side holes change by $7 \%$ or less, which would be expected to alter film effectiveness by of order 0.005 .

3. Vane coolant migration accounts for the effect of blade row interaction on pressureside film effectiveness. The wake negative jet transports coolant in the direction of the suction side, and so increases the time-averaged temperature on the pressure side above the mixed-out inlet value. In terms of a (stagnation) effectiveness, the local main-stream temperature on the pressure side is between -0.025 and -0.045 , consistent with the observed reduction in (adiabatic wall) film effectiveness.

4. The effect of blade row interaction on suction-side film effectiveness is due to a periodic increase in film mixing rate, by a factor of 2.5 , as vane wakes impinge on the blade surface. Arrival of a wake causes an increase in main-stream turbulence intensity to $T u_{\infty} \approx 5 \%$, and a reduction in film effectiveness of up to 0.19 or $60 \%$. This reduction is greater than would be expected from reported measurements of the effect of main-stream turbulence in flat plate experiments. Between wakes, the film effectiveness recovers to that in the mixing-plane simulation without blade row interactions.

5. The quasi-steady hole response model described in Chapter 6, when applied to the sliding-plane computational results, gives good predictions of ensemble-averaged 
momentum flux ratio. The model predictions match the resolved values to within a typical 5\%, validating the quasi-steady assumption for discharge coefficient and the applicability of the model. 



\section{Chapter 9}

\section{Conclusions and recommendations for future work}

\subsection{Conclusions}

The investigation described in this thesis set out to understand the effect of blade row interaction on rotor film cooling. Experimental tests in a simplified geometry were used to establish the general principles governing the effect of unsteadiness on film cooling performance, and a combination of low- and high-fidelity computations were used to study blade row interactions in representative geometry and boundary conditions. In this section, the key contributions of the work are summarised. The findings are presented in three parts, corresponding to the three research questions in Section 1.2.

\subsubsection{Fluid-dynamic mechanisms}

Research question 1: What are the fluid-dynamic mechanisms that characterise blade row interaction effects on rotor film cooling?

Quasi-steady hole flow An analysis of the relative time scales of rotor film cooling and vane passing, representative of a large industrial gas turbine, yields a reduced frequency of $\kappa_{\mathrm{c}} \approx 0.06$. This is a low value, and therefore rotor cooling holes may be expected to behave quasi-steadily, Section 2.4.1. The quasi-steady assumption is supported by the hot-wire measurements presented in Section 5.4, where phase-locked ensemble-averaged snapshots of film cooling with main-stream unsteadiness correspond to steady data at the instantaneous momentum flux ratio. The quasi-steady assumption 
is also supported by the accuracy of the quasi-steady hole response model, verified in Section 8.5.1 to predict unsteady momentum flux ratio to within a typical $5 \%$.

Non-linearity in hole response At constant time-averaged boundary condition, unsteadiness will affect film cooling performance if the hole responds non-linearly. This is demonstrated in Chapter 5, using film effectiveness measurements with and without main-stream static pressure fluctuations. With cylindrical holes in Section 5.3.1, film effectiveness is not affected by main-stream unsteadiness for momentum flux ratios where coolant has separated from the surface, $I R \geq 1$, because film effectiveness varies linearly with momentum flux ratio perturbations. In contrast, at an attached momentum flux ratio near peak film effectiveness, $I R=0.2$, unsteadiness reduces film effectiveness by up to 0.06 or $31 \%$, because the hole behaves non-linearly. A reduction in momentum flux ratio reduces coolant mass flow, while an increase in momentum flux ratio causes coolant separation, both of which act to reduce film effectiveness. With laid-back fan-shaped holes in Section 5.3.2, main-stream unsteadiness produces a consistent but smaller drop in effectiveness of between 0.01 and 0.03 , or up to $15 \%$. However, the data show that the steady film effectiveness-momentum flux ratio characteristic is linear at all operating points. Instead, it is hypothesised that diffusing, separated flow within the expanded hole exit has a longer characteristic time scale, resulting in a second-order non-quasi-steady reduction in performance.

Unsteady main-stream boundary conditions In Chapter 6, non-film-resolved computations are used to identify the blade row interaction mechanisms generating unsteady main-stream boundary conditions in a turbine stage. Section 6.3 shows that the upstream vane wake creates a negative jet velocity perturbation, and the upstream vane potential field creates pressure perturbations which propagate through the rotor passage as approximately one-dimensional waves. Computations with modified upstream vanes, in Section 6.4, show that both mechanisms contribute to unsteadiness in momentum flux ratio, of not less than $\pm 30 \%$ at all hole locations, but wake interactions produce greater perturbations by a factor of two to five.

Change in time-averaged boundary conditions In addition to unsteady fluctuations, blade row interaction may also modify the time-averaged boundary conditions imposed on rotor cooling holes, altering film cooling performance. Vane coolant migration, where cold fluid within the vane wake is carried towards the rotor suction side by the negative jet, is observed in three-dimensional URANS computations in Section 6.3.1, and 
hybrid URANS-LES turbine stage cascade computations in Section 8.3.4. In both cases, main-stream stagnation temperature variations are equivalent to \pm 0.03 in blade film effectiveness. If vane wakes are allowed to convect through the rotor, instead of being mixed out, blade row interaction increases the time-averaged turbulence intensity. The URANS-LES turbine stage cascade computations predict an increase in time-averaged turbulence intensity to $T u_{\infty} \approx 2 \%$, in Section 8.4.1.

\subsubsection{Effect on cooling performance}

Research question 2: How is the time-averaged unsteady film cooling different from the nominal steady film cooling?

General principles Knowledge of the physical mechanisms involved allow some general statements to be made on the effects of blade row interaction at turbine conditions. If the response of the cooling to main-stream unsteadiness is linear, then the effect of blade row interaction is only due to change in time-averaged boundary conditions. That is, the cooling performance at unsteady conditions is the same as a steady flow with the same time-averaged boundary conditions. The film effectiveness data of Chapter 5 suggest that separated cylindrical holes and laid-back fan-shaped holes would behave in this way. However, if the response of the cooling to main-stream unsteadiness is non-linear, then instantaneous variations must also be accounted for. If the flow is taken as quasi-steady, the time-averaged unsteady performance is the average over a vane passing period of a set of steady flows with the instantaneous boundary conditions.

Film-resolved cascade simulations In Chapter 8, a hybrid URANS-LES computational method is used to simulate the flow in a turbine stage linear cascade with resolved rotor cooling holes. The cascade is representative of the mid-span of the first high-pressure turbine stage of a large industrial gas turbine. Comparing two configurations, with uniform (mixed-out) and non-uniform (unsteady) rotor inflow, but the same geometry, flow solver and boundary conditions, allows a virtual experiment into the effect of blade row interaction on rotor film cooling. On the rotor pressure side, blade row interaction reduces laterally-averaged film effectiveness by up to 0.04 , due to vane coolant migration. The effect of blade row interaction is greater on the rotor suction side, with a film effectiveness reduction of up to 0.08. Ensemble-averaged data show that this is due to a periodic increase in film mixing rate, by a factor of 2.5 , as the vane wake impinges on the suction side. 


\subsubsection{Modelling}

Research question 3: How should blade row interaction effects on rotor film cooling be modelled?

Hybrid URANS-LES for film cooling In this project, a hybrid URANS-LES method has been developed for predicting film cooling performance subject to steady or unsteady main-stream boundary conditions. The method is validated against literature flat-plate film effectiveness data in Chapter 7. Predictions of laterally-averaged film effectiveness downstream of cylindrical cooling holes are accurate to within 21\%, Section 7.3.2. Accuracy is found to not be a strong function of URANS layer thickness. Using the same computational approach, as calibrated for cylindrical holes, for a laid-back fan-shaped hole test case also yields predictions of laterally-averaged film effectiveness of a similar accuracy, to within 23\%, Section 7.4.2. These results increase confidence in the general predictive capability of the method. Application of the method to a turbine stage cascade with resolved rotor cooling holes in Chapter 8 illustrates its utility for predicting film effectiveness in more realistic geometries with unsteady boundary conditions.

Quasi-steady hole model It is shown in Chapter 5 that unsteadiness in momentum flux ratio affects time-averaged film cooling performance if the fluctuations occur over a non-linear region of the hole characteristic. Therefore, it is of interest for a designer to predict the range of unsteady excursions in momentum flux ratio, and identify a potentially detrimental situation like this, without the computational expense of resolving the film cooling holes. Section 6.2 describes a modelling framework to achieve this aim. Unsteady main-stream boundary conditions are taken from URANS computations, without resolved cooling holes, and supplied as input to a quasi-steady discharge coefficient hole model to determine the resultant instantaneous momentum flux ratio. The model is validated against resolved cooling hole computations in Section 8.5.1, showing agreement to within a typical 5\%. The quasi-steady model allows the designer to target a region of the hole characteristic with a linear film effectiveness response. 


\subsection{Recommendations for future work}

In this section, based on the results presented in the thesis, suggestions are made for further work that could be undertaken.

Effect of unsteadiness on heat transfer coefficient All simulations discussed in this thesis have used adiabatic walls, and no attempt has been made to predict heat transfer coefficients. In the experiments, heat transfer coefficient data is only available in regions with coolant coverage, and so laterally-averaged distributions cannot be evaluated. It is the view of the author, based on the limited reported experimental data, that the heat transfer coefficient will respond linearly to main-stream unsteadiness, so any blade row interaction effects will be due to the change in time-averaged turbulence intensity alone. The computational or experimental methods would need to be modified to assess this conjecture.

Quasi-steady behaviour limits The current work has used a reduced frequency representative of a large industrial gas turbine, a low value of $\kappa_{\mathrm{c}} \approx 0.06$, meaning that to a close approximation the cooling hole flow may be taken as quasi-steady. However, in aero-engines, the reduced frequency is higher, $\kappa_{\mathrm{c}} \approx 0.2$, due to faster shaft speeds, and it is of interest whether the conclusions of this work are directly transferable. The influence of the amplitude and shape of the unsteady perturbation on the validity of the quasi-steady assumption have also not been investigated; generally, the local temporal gradient of main-stream unsteadiness should be taken into account as proposed by Cao et al. (2014). The experimental apparatus is suitable for answering these questions, with freedom to set the unsteadiness frequency over a wide range using the inverter drive, and some flexibility in unsteadiness amplitude by changing the pressure fluctuator rotating blockage size.

Effect of long length scale main-stream turbulence The computations in Chapter 8 suggest that wake interaction produces long length scale main-stream turbulence in the rotor passage. The integral length scale at the suction-side injection location is $\Lambda / D=7.6$, over twice the largest length scale tested in the film cooling literature. It is of interest whether the existing understanding of main-stream turbulence effects on film cooling performance, based on short turbulent length scales, is applicable to the predicted wake interactions. One way to determine this would be to add a crossflow jet inlet turbulence generator to the UFC Rig, and perform film effectiveness measurements with various turbulence intensities and length scales. 
Aerodynamics of diffusing holes The measurements of laid-back fan-shaped holes in Chapter 5 show a consistent drop in film effectiveness due to main-stream unsteadiness, which is not explained by non-linearity of the steady film effectiveness characteristic. However, for $I R \geq 0.6$, the reduction is of the same order as the experimental uncertainty. It is hypothesised that the reduction in cooling performance is due to a non-quasi-steady effect on the unstable, diffusing flow within the shaped cooling hole. Reducing the experimental film effectiveness uncertainty would be the first step to confirming the hypothesis. Then the investigation might proceed with additional measurements of the hole flow field, complemented by a more detailed examination of flat plate simulation results.

Hybrid URANS-LES method for film cooling The computations presented in Chapter 7 have demonstrated that, even with a crude wall model, hybrid URANSLES can can successfully predict film effectiveness across multiple geometries and flow conditions. However, given the sensitivity of blade creep life to metal temperatures, accuracy improvements would be beneficial. First, the lack of main-stream turbulence must be addressed to remove a source of error when comparing to experimental data. Second, rigorous grid and time-step independence studies are beyond the scope of this work, but should be carried out if the method is to be applied more widely. The mixinglength model must be modified to reduce turbulent viscosity in the laminar sub-layer, either as an explicit function of $y^{+}$or by scaling against a suitable combination of operators as in the WALE sub-grid scale model (Nicoud and Ducros, 1999). Finally, after these steps, more complex wall models such as the Spalart-Allmaras model may be investigated.

Film cooling virtual experiments A validated computational model for film cooling presents an opportunity to gather information on flow behaviour in more detail and with more precise control over boundary conditions than is feasible in a laboratory. For example, Mach number, Reynolds number and boundary layer thickness can only be decoupled in a closed-loop wind tunnel with some mechanical system for generating a boundary layer of variable thickness, whereas these parameters are straightforward to change in a computation. With the tools developed in this project, film effectiveness predictions for a new hole geometry can be generated within one week, including meshing and computation time.

Low-order modelling of instantaneous film effectiveness In Chapter 6, a quasisteady model is formulated to calculate the instantaneous momentum flux ratio of rotor 
cooling holes. No attempt is made to evaluate the instantaneous cooling performance in the low-order framework, because of the extra assumptions involved. However, the quasi-steady assumption for film effectiveness could be checked by imposing unsteady boundary conditions on the flat plate cooling hole cases, and comparing the unsteady film effectiveness with steady results. If it can be established that the film effectiveness responds quasi-steadily during unsteady excursions in operating point, instantaneous cooling performance can then be estimated without computationally-expensive film resolved simulations; only main-stream boundary conditions from non-film resolved simulations would be required. This would bring an optimisation study within reach, where the aerodynamic design of the turbine is modified to mitigate blade row interaction effects and improve rotor cooling performance. 



\section{References}

Abernethy, R., Benedict, R., and Dowdell, R. (1985). ASME Measurement Uncertainty. J. Fluids Eng., 107(2):161-164.

Abhari, R. S. (1996). Impact of Rotor-Stator Interaction on Turbine Blade Film Cooling. J. Turbomach., 118(1):123-133.

Abhari, R. S. and Epstein, A. H. (1994). An Experimental Study of Film Cooling in a Rotating Transonic Turbine. J. Turbomach., 116(1):63-70.

Aoki, S. (1999). Application of Aero-engine Technologies to Large Industrial Gas Turbines. ISABE Paper No. 99-7148.

ASME (2018). ASME Turbo Expo: Turbomachinery Technical Conference and Exposition. June 11-15, 2018, Oslo, Norway. Volume 5: Heat Transfer.

Atkins, N. R. and Kanjirakkad, V. (2014). Flow in a Rotating Cavity with Axial Throughflow at Engine Representative Conditions. ASME Paper No. 2014-27174.

Baldauf, S. and Scheurlen, M. (1996). CFD Based Sensitivity Study of Flow Parameters for Engine Like Film Cooling Conditions. ASME Paper No. 96-GT-310.

Baldauf, S., Scheurlen, M., Schulz, A., and Wittig, S. (2002). Correlation of FilmCooling Effectiveness From Thermographic Measurements at Enginelike Conditions. J. Turbomach., 124(4):686-698.

Bearman, P. (1971). Corrections for the Effect of Ambient Temperature Drift on Hot-wire Measurements in Incompressible Flow. DISA Information Report No. 11.

Bernsdorf, S., Rose, M. G., and Abhari, R. S. (2008). Experimental Validation of Quasisteady Assumption in Modeling of Unsteady Film-Cooling. J. Turbomach., 130(1):011022.

Bidan, G., Vézier, C., and Nikitopoulos, D. E. (2013). Study of Unforced and Modulated Film-Cooling Jets Using Proper Orthogonal Decomposition-Part I: Unforced Jets. J. Turbomach., 135(2):021037.

Binder, A. (1985). Turbulence Production due to Secondary Vortex Cutting in a Turbine Rotor. J. Eng. Gas Turbines Power, 107(4):1039-1046.

Bogard, D. G. and Thole, K. A. (2006). Gas Turbine Film Cooling. J. Propul. Power, $22(2): 249-270$. 
Borup, D. D., Fan, D., Elkins, C. J., and Eaton, J. K. (2018). Experimental Study of Periodic Free Stream Unsteadiness Effects on Discrete Hole Film Cooling in Two Geometries. ASME Paper No. 2018-75836.

Bose, S. T., Moin, P., and You, D. (2010). Grid-independent large-eddy simulation using explicit filtering. Phys. Fluids, 22(10):105103.

Brandvik, T. and Pullan, G. (2011). An Accelerated 3D Navier-Stokes Solver for Flows in Turbomachines. J. Turbomach., 133(2):021025.

Bunker, R. (2017). Evolution of Turbine Cooling. IGTI Scholar Lecture, ASME Paper No. GT2017-63205.

Butler, T., Sharma, O., Joslyn, H., and Dring, R. (1989). Redistribution of an Inlet Temperature Distortion in an Axial Flow Turbine Stage. J. Propul. Power, 5(1):64-71.

Cao, T., Xu, L., Yang, M., and Martinez-Botas, R. F. (2014). Radial Turbine Rotor Response to Pulsating Inlet Flows. J. Turbomach., 136(7):071003.

Cumpsty, N. A. (2003). Jet Propulsion. Cambridge University Press.

Day, C., Oldfield, M., and Lock, G. (2000). Aerodynamic performance of an annular cascade of film cooled nozzle guide vanes under engine representative conditions. Exp. Fluids, 29(2):117-129.

Dean, R. C. (1959). On the Necessity of Unsteady Flow in Fluid Machines. J. Basic Eng., 81(1):24-28.

Denton, J. D. (1992). The Calculation of Three-Dimensional Viscous Flow Through Multistage Turbomachines. J. Turbomach., 114(1):18-26.

Denton, J. D. (2002). The Effects of Lean and Sweep on Transonic Fan Performance. Task Quarterly, 6(1):7-23.

Denton, J. D. (2017). Multall-An Open Source, Computational Fluid Dynamics Based, Turbomachinery Design System. J. Turbomach., 139(12):121001.

Dominy, R. and Hodson, H. (1993). An Investigation of Factors Influencing the Calibration of Five-hole Probes for Three-dimensional Flow Measurements. J. Turbomach., 115(3):513-519.

Dring, R., Blair, M., and Joslyn, H. (1980). An Experimental Investigation of Film Cooling on a Turbine Rotor Blade. J. Eng. Power, 102(1):81-87.

Du, H., Ekkad, S. V., and Han, J.-C. (1999). Effect of Unsteady Wake With Trailing Edge Coolant Ejection on Film Cooling Performance for a Gas Turbine Blade. J. Turbomach., 121(3):448-455.

Du, H., Han, J., and Ekkad, S. (1998). Effect of Unsteady Wake on Detailed Heat Transfer Coefficient and Film Effectiveness Distributions for a Gas Turbine Blade. J. Turbomach., 120(4):808-817. 
Ferguson, J. D., Walters, D. K., and Leylek, J. H. (1998). Performance of Turbulence Models and Near-wall Treatments in Discrete Jet Film Cooling Simulations. ASME Paper No. 98-GT-438.

Foroutan, H. and Yavuzkurt, S. (2015). Numerical Simulations of the Near-Field Region of Film Cooling Jets Under High Free Stream Turbulence: Application of RANS and Hybrid URANS/Large Eddy Simulation Models. J. Heat Transfer, 137(1):011701.

Fujimoto, S. (2012). Large Eddy Simulation of Film Cooling Flows Using Octree Hexahedral Meshes. ASME Paper No. GT2012-70090.

Funazaki, K., Yokota, M., and Yamawaki, S. (1997). Effect of Periodic Wake Passing on Film Effectiveness of Discrete Cooling Holes Around the Leading Edge of a Blunt Body. J. Turbomach., 119(2):292-301.

Giles, M. B. (1990). Stator/rotor Interaction in a Transonic Turbine. J. Propul. Power, $6(5): 621-627$.

Greitzer, E. M., Tan, C., and Graf, M. (2004). Internal Flow: Concepts and Applications. Cambridge University Press, Cambridge, UK.

Grimshaw, S. and Taylor, J. (2016). Fast Settling Millimetre-scale Five-hole Probes. ASME Paper No. GT2016-56628.

Gritsch, M., Schulz, A., and Wittig, S. (1998). Method for Correlating Discharge Coefficients of Film-Cooling Holes. AIAA J., 36(6):976-980.

Guo, X., Schröder, W., and Meinke, M. (2006). Large-eddy Simulations of Film Cooling Flows. Comput. Fluids, 35(6):587-606.

Han, J.-C. (2004). Recent Studies in Turbine Blade Cooling. Int. J. Rotating Mach., $10(6): 443-457$.

Harrison, K. L. and Bogard, D. G. (2008). Comparison of RANS Turbulence Models for Prediction of Film Cooling Performance. ASME Paper No. GT2008-51423.

Heidmann, J. D., Lucci, B. L., and Reshotko, E. (2001). An Experimental Study of the Effect of Wake Passing on Turbine Blade Film Cooling. J. Turbomach., 123(2):214-221.

Hodson, H. P. (2017). Personal communication.

Hodson, H. P. and Dawes, W. N. (1998). On the Interpretation of Measured Profile Losses in Unsteady Wake-Turbine Blade Interaction Studies. J. Turbomach., 120(2):276-284.

Hunt, J. C., Wray, A. A., and Moin, P. (1988). Eddies, Streams, and Convergence Zones in Turbulent Flows. Center for Turbulence Research Report CTR-S88.

International Energy Agency (2018). World Energy Outlook. Technical report.

Ireland, P. T. (2014). Turbine Heat Transfer and Cooling. Centre for Doctoral Training in Gas Turbine Aerodynamics Lecture Series, University of Cambridge. 
Jameson, A. (1991). Time Dependent Calculations Using Multigrid, with Applications to Unsteady Flows Past Airfoils and Wings. AIAA Paper No. 91-159\%.

Johnson, R., Maikell, J., Bogard, D., Piggush, J., Kohli, A., and Blair, M. (2009). Experimental Study of the Effects of an Oscillating Approach Flow on Overall Cooling Performance of a Simulated Turbine Blade Leading Edge. ASME Paper No. 2009-GT-60290.

Kachel, C. E. and Denton, J. D. (2006). Experimental and Numerical Investigation of the Unsteady Surface Pressure in a Three-Stage Model of an Axial High Pressure Turbine. J. Turbomach., 128(2):261-272.

Kays, W. M. (1994). Turbulent Prandtl number-Where Are We? J. Heat Transfer, 116(2):284-295.

Kerrebrock, J. L. and Mikolajczak, A. (1970). Intra-stator Transport of Rotor Wakes and Its Effect on Compressor Performance. J. Eng. Power, 92(4):359-368.

Kim, S. I. and Hassan, I. (2010). Unsteady Simulations of a Film Cooling Flow from an Inclined Cylindrical Jet. J. Thermophys. Heat Transfer, 24(1):145-156.

Klostermeier, C. (2008). Investigation Into the Capability of Large Eddy Simulation for Turbomachinery Design. PhD thesis, University of Cambridge.

Konopka, M., Jessen, W., Meinke, M., and Schröder, W. (2013). Large-eddy Simulation of Film Cooling in an Adverse Pressure Gradient Flow. J. Turbomach., 135(3):031031.

Leedom, D. and Acharya, S. (2008). Large Eddy Simulations of Film Cooling Flow Fields from Cylindrical and Shaped Holes. ASME Paper No. GT2008-51009.

Liess, C. (1975). Experimental Investigation of Film Cooling with Ejection from a Row of Holes for the Application to Gas Turbine Blades. J. Eng. Power, 97(1):21-27.

Ligrani, P., Gong, R., Cuthrell, J., and Lee, J. (1996). Bulk flow pulsations and film cooling-I. Injectant behavior. Int. J. Heat Mass Transfer, 39(11):2271-2282.

Lim, C. H., Pullan, G., and Ireland, P. (2013). Influence of Film Cooling Hole Angles and Geometries on Aerodynamic Loss and Net Heat Flux Reduction. J. Turbomach., 135(5):051019.

Luque, S., Jones, T., and Povey, T. (2016). Theory for the scaling of metal temperatures in cooled compressible flows. Int. J. Heat Mass Transfer, 102:331-340.

Mehendale, A. B., Han, J.-C., Ou, S., and Lee, C. (1994). Unsteady Wake Over a Linear Turbine Blade Cascade With Air and $\mathrm{CO}_{2}$ Film Injection: Part II-Effect on Film Effectiveness and Heat Transfer. J. Turbomach., 116(4):730-737.

Meyer, R. (1958). The Effect of Wakes on the Transient Pressure and Velocity Distributions in Turbomachines. J. Basic Eng., 80(7):1544-1552.

Miller, R., Moss, R., Ainsworth, R., and Harvey, N. (2003). Wake, Shock, and Potential Field Interactions in a 1.5 Stage Turbine - Part I: Vane-rotor and Rotor-vane Interaction. J. Turbomach., 125(1):33-39. 
Muldoon, F. and Acharya, S. (2006). Analysis of $k-\epsilon$ Budgets for Film Cooling using Direct Numerical Simulation. AIAA J., 44(12):3010-3021.

Nicoud, F. and Ducros, F. (1999). Subgrid-Scale Stress Modelling Based on the Square of the Velocity Gradient Tensor. Flow Turbul. Combust., 62(3):183-200.

Numeca International (2014). IGG9.1-3/AUTOGRID5. www. numeca.com.

O'Dowd, D., Zhang, Q., He, L., Ligrani, P., and Friedrichs, S. (2011). Comparison of Heat Transfer Measurement Techniques on a Transonic Turbine Blade Tip. J. Turbomach., 133(2):021028.

Oldfield, M. (2008). Impulse Response Processing of Transient Heat Transfer Gauge Signals. J. Turbomach., 130(2):021023.

Oliver, T. A., Anderson, J. B., Bogard, D. G., Moser, R. D., and Laskowski, G. (2017). Implicit LES for Shaped-Hole Film Cooling Flow. ASME Paper No. 2017-63314.

Ong, J. and Miller, R. J. (2012). Hot Streak and Vane Coolant Migration in a Downstream Rotor. J. Turbomach., 134(5):051002.

Peet, Y. V. and Lele, S. K. (2008). Near Field of Film Cooling Jet Issued into a Flat Plate Boundary Layer: LES Study. ASME Paper No. GT2008-50420.

Playford, W. (2018). Well-Conditioned Heat Transfer Measurements on Engine Scale Gas Turbine Rigs. PhD thesis, University of Cambridge.

Program Development Company (2017). GRIDPRO v6.6. www.gridpro.com.

Pullan, G. (2006). Secondary Flows and Loss caused by Blade Row Interaction in a Turbine Stage. J. Turbomach., 128(3):484-491.

Rajagopalan, K. and Eiseman, P. R. (2005). Automatic nested refinement: a technique for the generation of high quality multi-block structured grids for multi-scale problems using GridPro. Eng. Comput., 21(1):29-35.

Renze, P., Schröder, W., and Meinke, M. (2008). Large-eddy simulation of film cooling flows at density gradients. Int. J. Heat Fluid Flow, 29(1):18-34.

Rowbury, D., Oldfield, M., and Lock, G. (2001). A Method for Correlating the Influence of External Crossflow on the Discharge Coefficients of Film Cooling Holes. J. Turbomach., $123(2): 258-265$.

Rutledge, J. L., Polanka, M. D., and Bogard, D. G. (2016). The Delta Phi Method of Evaluating Overall Film Cooling Performance. J. Turbomach., 138(7):071006.

Saumweber, C. and Schulz, A. (2012). Free-Stream Effects on the Cooling Performance of Cylindrical and Fan-Shaped Cooling Holes. J. Turbomach., 134(6):061007.

Saumweber, C., Schulz, A., and Wittig, S. (2003). Free-Stream Turbulence Effects on Film Cooling With Shaped Holes. J. Turbomach., 125:65-73. 
Schmidt, D. and Bogard, D. (1996). Effects of Free-Stream Turbulence and Surface Roughness on Film Cooling. ASME Paper No. 96-GT-462.

Sen, B., Schmidt, D. L., and Bogard, D. G. (1996). Film Cooling with Compound Angle Holes: Heat Transfer. J. Turbomach., 118(4):800-806.

Seo, H. J., Lee, J. S., and Ligrani, P. M. (1999). Effects of Bulk Flow Pulsations on Film Cooling From Different Length Injection Holes at Different Blowing Ratios. J. Turbomach., 121(3):542-550.

Sinha, A. K., Bogard, D. G., and Crawford, M. E. (1991). Film-Cooling Effectiveness Downstream of a Single Row of Holes With Variable Density Ratio. J. Turbomach., 113(3):442-449.

Spalart, P. and Allmaras, S. (1992). A one-equation turbulence model for aerodynamic flows. AIAA J., 94.

Spalart, P. R. (2009). Detached-Eddy Simulation. Annu. Rev. Fluid Mech., 41:181-202.

Takeishi, K., Aoki, S., Sato, T., and Tsukagoshi, K. (1992). Film Cooling on a Gas Turbine Rotor Blade. J. Turbomach., 114(4):828-834.

Thole, K., Gritsch, M., Schulz, A., and Wittig, S. (1998). Flowfield Measurements for Film-Cooling Holes With Expanded Exits. J. Turbomach., 120(2):327-336.

Thole, K., Sinha, A., Bogard, D., and Crawford, M. (1992). Mean Temperature Measurements of Jets with a Crossflow for Gas Turbine Film Cooling Application. In Rotating Machinery-Transport Phenomena, volume 1, pages 69-85.

Tucker, P., Eastwood, S., Klostermeier, C., Jefferson-Loveday, R., Tyacke, J., and Liu, Y. (2012a). Hybrid LES Approach for Practical Turbomachinery Flows - Part I: Hierarchy and Example Simulations. J. Turbomach., 134(2):021023.

Tucker, P., Eastwood, S., Klostermeier, C., Xia, H., Ray, P., Tyacke, J., and Dawes, W. (2012b). Hybrid LES Approach for Practical Turbomachinery Flows - Part II: Further Applications. J. Turbomach., 134(2):021024.

Tucker, P. G. (2013). Unsteady Computational Fluid Dynamics in Aeronautics, volume 104. Springer Science \& Business Media.

Tyagi, M. and Acharya, S. (2003). Large Eddy Simulation of Film Cooling Flow from an Inclined Cylindrical Jet. J. Turbomach., 125(4):734-742.

Walters, D. and Leylek, J. (1997). A Systematic Computational Methodology Applied to a Three-Dimensional Film-Cooling Flowfield. J. Turbomach., 119(4):777-785.

Walters, D. K. and Leylek, J. H. (2000). Impact of Film-Cooling Jets on Turbine Aerodynamic Losses. J. Turbomach., 122(3):537-545.

Wilcock, R., Young, J., and Horlock, J. (2005). The Effect of Turbine Blade Cooling on the Cycle Efficiency of Gas Turbine Power Cycles. J. Eng. Gas Turbines Power, 127(1):109-120. 
Wittig, S., Schulz, A., Gritsch, M., and Thole, K. (1996). Transonic Film-Cooling Investigations: Effects of Hole Shapes and Orientations. ASME Paper No. 96-GT-222.

Womack, K. M., Volino, R. J., and Schultz, M. P. (2008). Measurements in Film Cooling Flows with Periodic Wakes. J. Turbomach., 130(4):041008.

Young, J. and Wilcock, R. (2002a). Modeling the Air-Cooled Gas Turbine: Part 1General Thermodynamics. J. Turbomach., 124(2):207-213.

Young, J. and Wilcock, R. (2002b). Modeling the Air-Cooled Gas Turbine: Part 2Coolant Flows and Losses. J. Turbomach., 124(2):214-221.

Yuri, M., Tsukagoshi, K., Hada, S., Masada, J., and Ito, E. (2013). Development of $1600^{\circ} \mathrm{C}$-Class High-efficiency Gas Turbine for Power Generation Applying J-Type Technology. Mitsubishi Heavy Industries Technical Review, 50(3).

Zhong, L., Zhou, C., and Chen, S. (2016). Large Eddy Simulation of Inclined Jet in Cross Flow with Cylindrical and Fan-shaped Holes. ASME Paper No. GT2016-56840.

Ziefle, J. and Kleiser, L. (2013). Numerical Investigation of a Film-Cooling Flow Structure: Effect of Crossflow Turbulence. J. Turbomach., 135(4):041001. 